
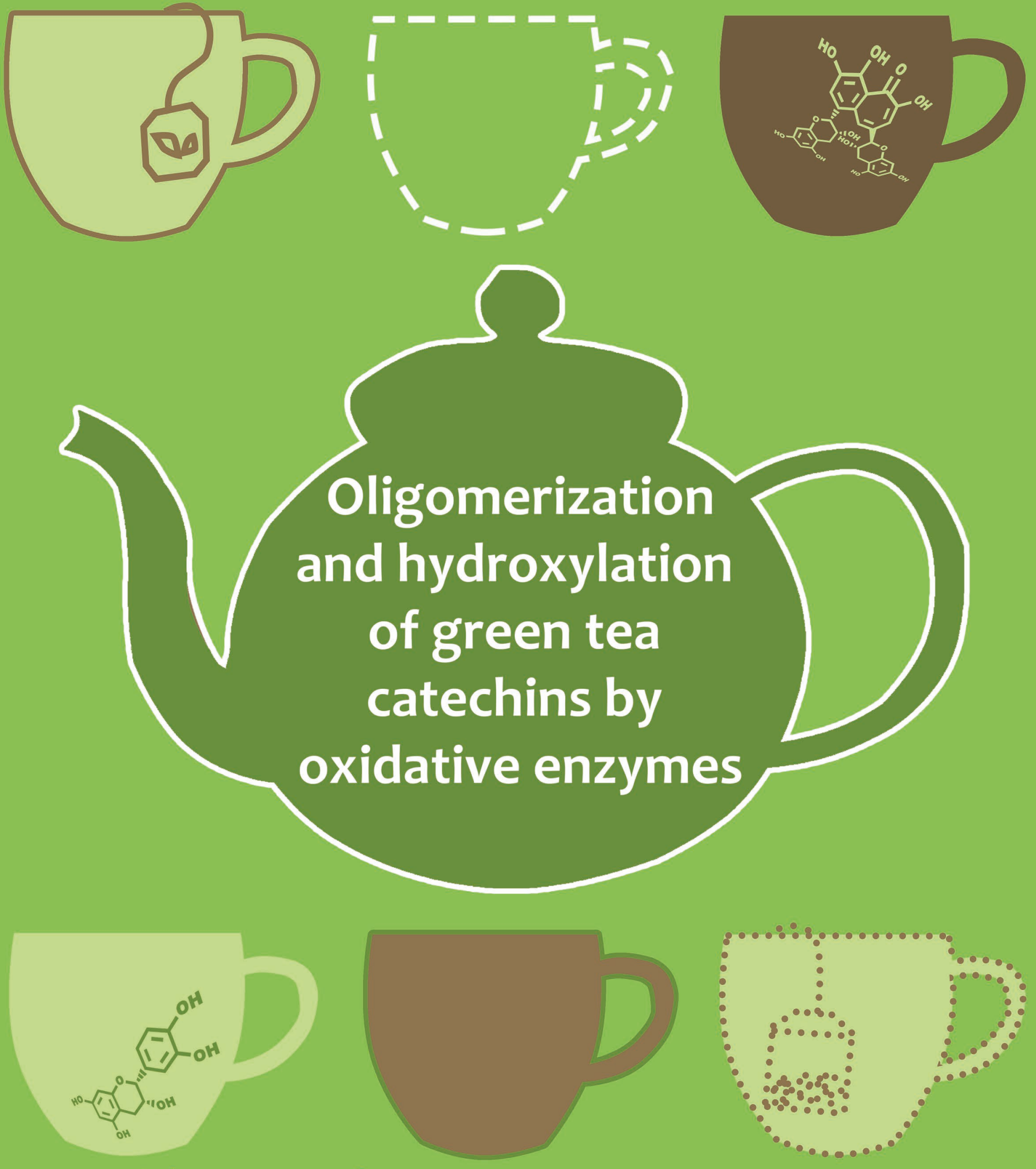

Annewieke J.W. Verloop
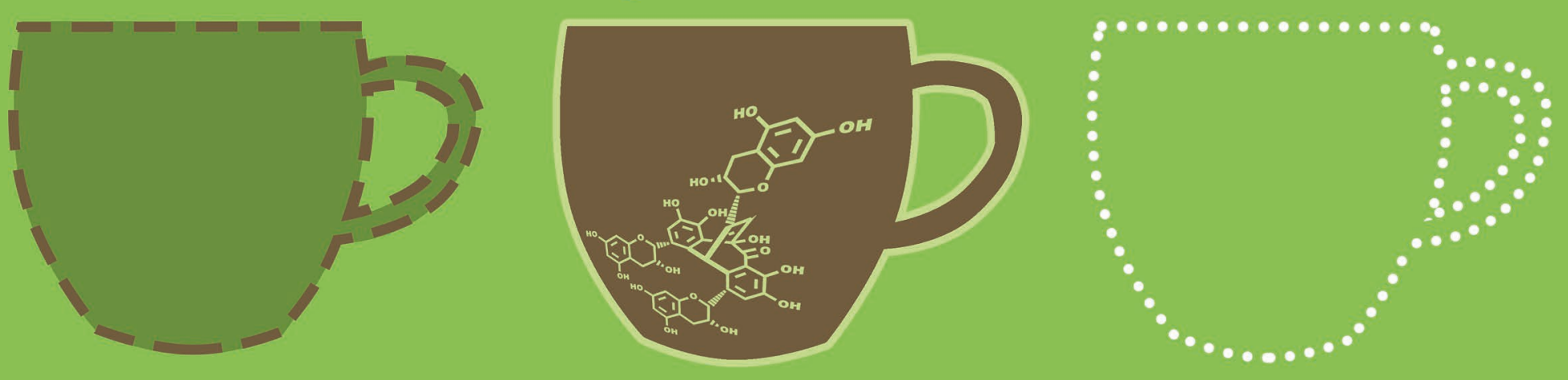
Oligomerization and hydroxylation of green tea catechins by oxidative enzymes

\author{
Annewieke J.W. Verloop
}




\section{Thesis committee}

\section{Promotor}

Prof. Dr H. Gruppen

Professor of Food Chemistry

Wageningen University

\section{Co-promotor}

Dr J.-P. Vincken

Associate professor, Laboratory of Food Chemistry

Wageningen University

\section{Other members}

Prof. Dr W.J.H. van Berkel, Wageningen University

Dr H.T.W.M. van der Hijden, Unilever R\&D, Vlaardingen, The Netherlands

Dr M. Dekker, Wageningen University

Dr W.M.A. Niessen, Hyphen MassSpec, Warmond, The Netherlands

This research was conducted under the auspices of the Graduate School VLAG (Advanced studies in Food Technology, Agrobiotechnology, Nutrition and Health Sciences). 


\title{
Oligomerization and hydroxylation of green tea catechins by oxidative enzymes
}

\author{
Annewieke J.W. Verloop
}

\author{
Thesis \\ submitted in fulfilment of the requirements for the degree of doctor \\ at Wageningen University \\ by the authority of the Rector Magnificus \\ Prof. Dr A.P.J. Mol, \\ in the presence of the \\ Thesis Committee appointed by the Academic Board \\ to be defended in public \\ on Friday 3 June 2016 \\ at 4 p.m. in the Aula.
}


Annewieke J.W. Verloop

Oligomerization and hydroxylation of green tea catechins by oxidative enzymes 146 pages.

$\mathrm{PhD}$ thesis, Wageningen University, Wageningen, NL (2016)

With references, with summary in English

ISBN 978-94-6257-777-0 


\section{Abstract}

Black teas are known for their characteristic brown colour, bitter taste and astringent mouth feel. These sensory characteristics are mainly influenced by the phenolic oxidation products present in black tea. The oxidation of phenolics from green tea leaves during black tea manufacturing is an uncontrolled process. With the objective to make tea oxidation a more controlled process, the aim of this thesis was to understand the enzymatic oxidation reactions occurring during tea oxidation, and to enable more rapid analysis of complex mixtures of phenolics. By incubating green tea catechins with an exogenous tyrosinase, a black tea-like phenolic profile was obtained, enriched in theaflavins, which are important for quality of tea. Further oxidation of theaflavins yielded theatridimensins, in which an epicatechin is coupled to the benzotropolone ring of theaflavin. By using MS/MS on selected ions these theatridimensins were shown to occur in black tea. This MS method could also be used to distinguish isomeric procyanidins and dehydrocatechins based on $\mathrm{MS}^{2}$ fragments, as well as the different interflavanic configurations occurring in dehydrodicatechins. The dehydrocatechins were shown to occur in black tea as well. Besides these oligomerization reactions mediated by tyrosinase, oxidation of tea phenolics also comprised hydroxylation. The enzymatic activity from tea leaves responsible for this hydroxylation reaction, was found to be peroxidase. All findings were condensed into a new version of the 'oxidative cascade hypothesis', describing the oxidation reactions towards formation of a black tea. 



\section{Table of contents}

Chapter 1

Chapter 2

Chapter 3

Chapter 4

Chapter 5

Chapter 6

Summary

Acknowledgements

An MS/MS method based on selected ions detects low abundance phenolics in black tea - theatridimensins as product of the oxidative cascade

Altering the phenolics profile of a green tea leaves extract using exogenous oxidases

Structural characterization of different dehydrocatechin oligomers by MS/MS, and their occurrence in black tea

Peroxidase is responsible for the hydroxylation step in the 'oxidative cascade' during processing of black tea

General discussion

About the author
1 

Chapter 1

General introduction 
The production of black tea is a thousands-of-years-old method that has hardly changed over the centuries. As simple as the production method seems, cutting and crushing tea leaves and leaving them to oxidize and dry, as complicated is the phenolic profile of a black tea. Up to 10,000 different phenolic compounds are present in a cup of black tea. ${ }^{l}$ The major reactions leading to their formation have fascinated scientists over the years. An essential part of the reactions are the oxidative enzymatic activities that catalyse the transformations. The role of the different oxidative enzymes in tea leaves, participating in different reaction pathways, is so far largely unknown. ${ }^{2}$

Both the oxidative enzymes and the phenolics initially present in the tea leaves, catechins, are key players in the black tea oxidation process. During conventional processing the natural morphological structure of the plant material is destroyed. As a result enzymes, catechins and oxygen get in contact. ${ }^{3}$ This is merely an uncontrolled process, which can only be influenced to a limited extent to modulate tea quality.

The enzymatic browning of tea might become more controlled if the key players, the oxidative enzymes and the catechins, would be separated in their native states, prior to processing. The oxidative enzymes should not be denatured and inactivated, whereas the catechins should be protected from oxidation. For such purpose, a 'mild' processing method should be developed. Further fractionation of the separate streams of oxidative enzymes and phenolics is also possible. This might yield pure enzyme activities or specific phenolic fractions. After the 'mild' separation, the enzymes and fractions of phenolics can be mixed to the desired ratios and conditions. This could, in the long run, potentially lead to an increased quality tea. To reach this, the different types of oxidative reactions should be elucidated. Also, the role of the different oxidative enzymes in black tea formation should be known.

\subsection{Composition of tea leaves}

Tea is the beverage obtained after (hot) water extraction of the leaves of Camellia sinensis. For tea production only the bud and the first two leaves from the top of the plant are used. ${ }^{4}$ The lower the leaves on the plant, the more mature they are, and the lower the content of catechins, ${ }^{5,6}$ an important quality parameter for teas. ${ }^{3,7}$ Different varieties of Camellia sinensis are used in tea production. The Camellia sinensis var. sinensis, mainly grown in China and other mildly cold climates in Asia, is used for green teas. The Camellia sinensis var. assamica, mainly grown in India and other semitropical climates, is used for black tea production. ${ }^{6,8}$ The difference between the two varieties lies mainly in the catechin content of the leaves. The assamica variety has a two times higher catechin content than the sinensis variety. ${ }^{6}$ Table 1.1 shows the average composition of the bud and the first two 
leaves of an assamica plant on a dry weight basis. The dry weight is approximately $25 \%$ of the fresh leaves. ${ }^{6}$

Table 1.1 Average composition* of the bud and the first two leaves from the top of Camellia sinensis var. assamica. ${ }^{\prime}$

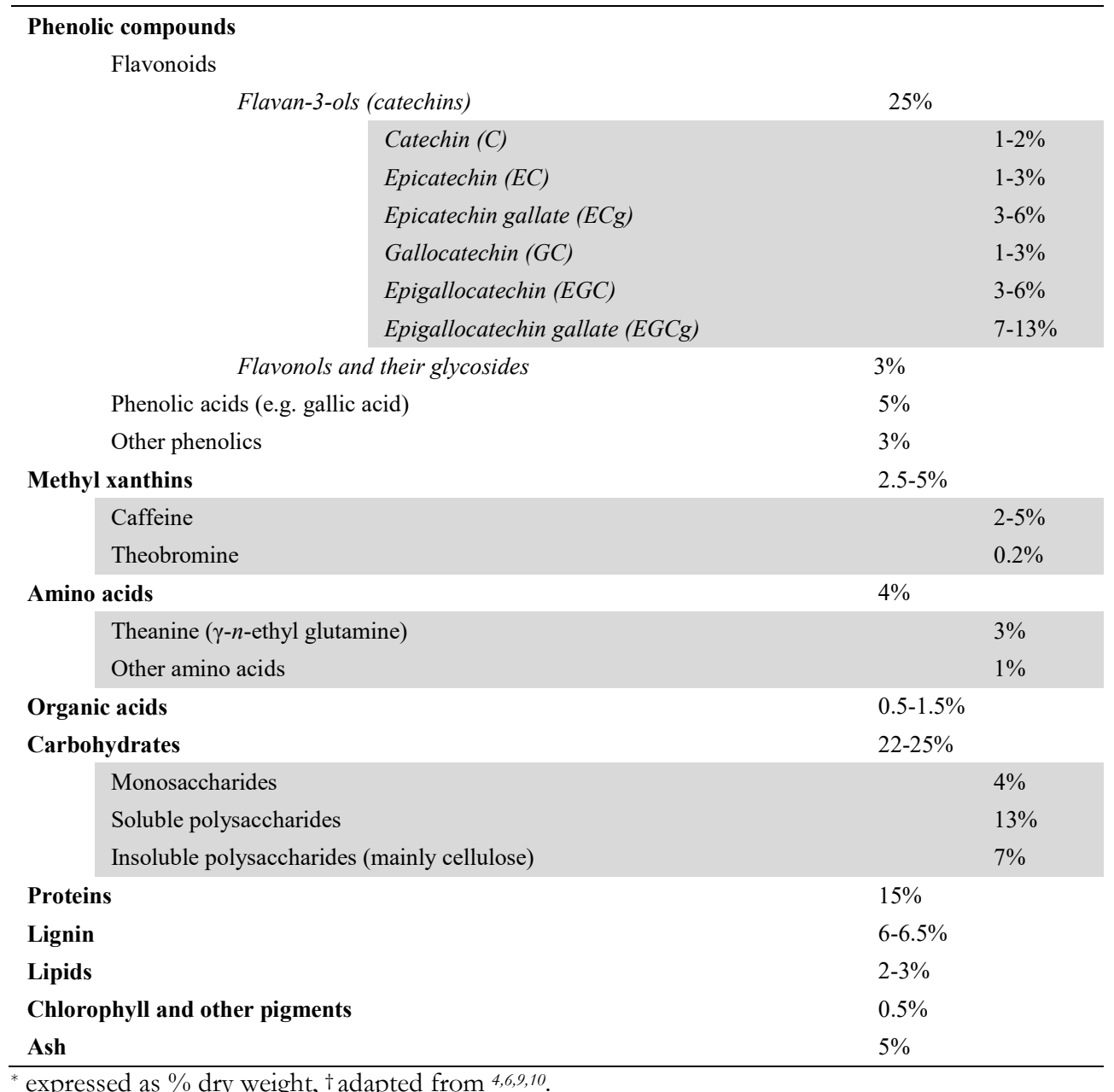

The phenolic compounds constitute $30-36 \%$, on dry weight (DW) basis, of the tea leaves, of which approximately 75\% comprises flavan-3-ols (2-phenyl-3,4-dihydro- $2 \mathrm{H}$ chromen-3-ols), also referred to as catechins. Epigallocatechin gallate (EGCg) is the main catechin species present in tea leaves, constituting almost $50 \%$ of the catechins. 
Epigallocatechin and epicatechin gallate are the next most abundant species, each constituting $12-25 \%$ of the catechins. Other phenolic compounds present in tea leaves are procyanidins, which also belong to the flavanol group (no separate percentage has been reported in literature), flavonols and their glycosides (mainly quercetin, kaempferol and myricetin), ${ }^{11,12}$ and phenolic acids, such as gallic acid and its quinic acid derivative, theogallin. Tea leaves contain $2-5 \% \mathrm{DW}(\mathrm{w} / \mathrm{w})$ of caffeine and about $0.2 \% \mathrm{DW}(\mathrm{w} / \mathrm{w})$ theobromine, another methyl xanthin. Other nitrogenous material in tea leaves are the proteins $(15 \% \mathrm{DW}(\mathrm{w} / \mathrm{w}))$, only a small part of which comprises the oxidative enzymes. Free amino acids $(4 \% \mathrm{DW}(\mathrm{w} / \mathrm{w}))$ are also present in tea leaves, of which theanine constitutes $75 \%$. Theanine ( $\gamma$ - $n$-ethyl glutamine) is an amino acid unique for tea leaves. Chlorophylls (also nitrogenous compounds) together with other pigments, mainly carotenoids, comprise $0.5 \% \mathrm{DW}(\mathrm{w} / \mathrm{w})$ of a tea leaf. Both soluble and insoluble carbohydrates are present $(22-25 \% \mathrm{DW}(\mathrm{w} / \mathrm{w}))$, which are responsible for the plant cell wall, together with lignin (6-6.5\% DW (w/w)). The lipids $(2-3 \% \mathrm{DW}(\mathrm{w} / \mathrm{w}))$ are mainly on the outside of the leaf, as the plant's cuticle wax. ${ }^{4,9}$

\subsection{Tea fermentation process}

Different kinds of tea can be obtained from a single variety by changing the fermentation process (Fig. 1.1). For tea, fermentation does not refer to a microbial treatment, but to a browning reaction catalysed by the endogenous enzymes of the tea plant. This process is better referred to as oxidation process. The process is initiated by disruption of the cell walls by rolling, cutting or bruising the leaves. The endogenous enzymes, located in the chloroplasts, ${ }^{13,14}$ come into contact with the catechins, located in the vacuoles, ${ }^{3,6,15,16}$ and oxygen, causing formation of several oxidation products.

The most common types of teas are white, green, oolong and black teas, which differ in their degree of oxidation (Fig. 1.1). The least processed form is white tea, where the freshly picked leaves are immediately dried after picking. The other kinds of tea require a withering step, in which the tea leaves are left after picking. During the withering step the leaves loose moisture and changes occur in cell membrane permeability. Also chemical changes occur important for flavour formation (e.g. linalool, geraniol). ${ }^{17} \mathrm{~A}$ green tea is obtained when tea leaves are heated and dried immediately after withering. Both the white and green teas are non-oxidized teas. After withering the leaves can also be bruised, rolled and/or cut, to initiate the oxidation process. An oolong tea is obtained when the tea leaves are heated and dried immediately after the bruising step. When the leaves are left to 'ferment' (oxidize), often combined with aeration of the leaves, a black tea is obtained. Black tea is the most oxidized type of tea. ${ }^{4,9}$ 


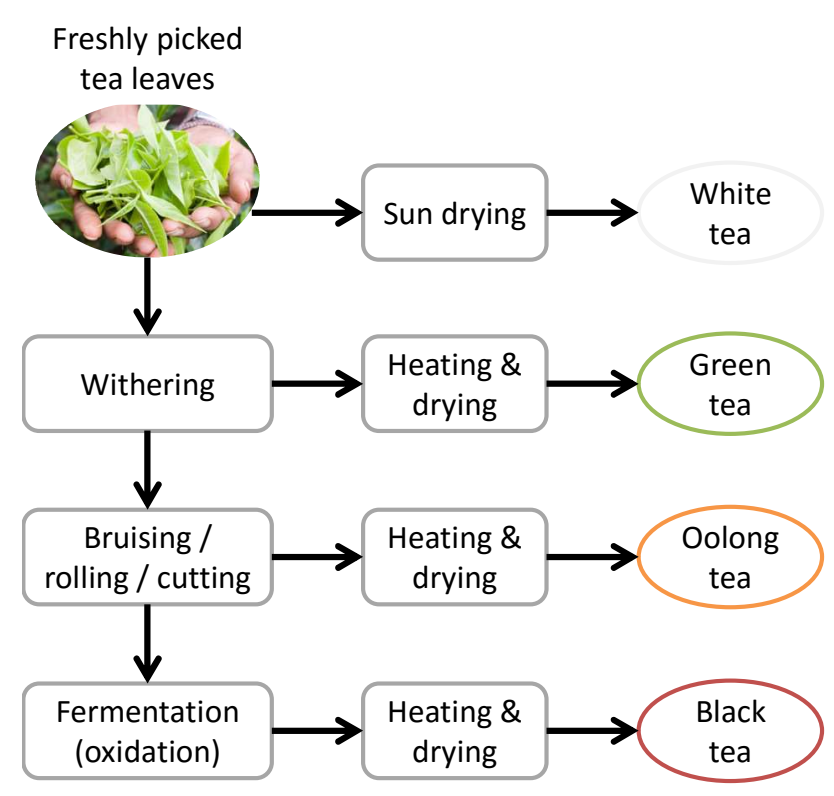

Figure 1.1 Flow chart showing the process towards different types of tea. ${ }^{4}$

Mixing tea leaves together with leaves from other plant species has been done before with loquat leaves $(10 \% \mathrm{w} / \mathrm{w})$. An accelerated oxidation and increased TF content was obtained, because of the strong oxidative activity of loquat leaf enzymes and because of the presence of caffeoyl quinic acids, the latter of which increased the oxidation by coupled oxidation reactions. ${ }^{18}$

The chemical composition of the different teas will mainly differ in their phenolic composition, as is shown for green tea versus black tea in Table 1.2. It should be considered that, as stated before, different varieties of Camellia sinensis are used for production of green and black teas, with a higher initial catechin content for black tea (var. assamica) than for green tea (var. sinensis). Green tea still has the non-oxidized catechins, whereas in black tea $85 \%$ of the catechins are oxidized into complex phenolic compounds, the theaflavins and thearubigins. ${ }^{9}$ The phenolic profile of a white tea is similar to that of a green tea, without significant differences in catechin content. ${ }^{19}$ Only the caffeine content showed to be 1.5 to 2 times higher for white teas than for green teas. ${ }^{20}$ In oolong tea, a two times higher content of non-oxidized catechins was still present in comparison to black tea, due to the shorter oxidation process. Consequently also $50 \%$ less theaflavins and $30 \%$ less thearubigins were found in the oolong tea. ${ }^{19}$ 
Table 1.2 Phenolic composition of a green tea and a black tea extract.*

\begin{tabular}{lcc}
\hline \multicolumn{2}{c}{$\%$ Total phenolics } \\
Green tea & Black tea \\
\hline Flavanols & & \\
\multicolumn{1}{c}{ Catechins } & $66-81 \%$ & $9-16 \%$ \\
Theaflavins & $0 \%$ & $6-10 \%$ \\
$\quad$ Thearubigins & $0 \%$ & $36-37 \%$ \\
Flavonols and their glycosides & $8-16 \%$ & $13-18 \%$ \\
Phenolic acids & $5-8 \%$ & $19-30 \%$ \\
Other phenolics & $5-10 \%$ & $5-9 \%$ \\
\hline
\end{tabular}

*adapted from $9,10,21$.

\subsection{Flavanolic compounds in tea}

In fresh green tea leaves, around $75 \%$ of the phenolic compounds are catechins. Catechins belong to the group of flavan-3-ols, characterized by their meta-5,7-dihydroxy substituted A-ring and di- or trihydroxylic B-ring (Fig. 1.2), the latter of which are referred to as 'gallocatechins'. Besides the number of hydroxyl groups substituted to the B-ring, the catechins can also differ in the occurrence of a gallic acid moiety esterified to the C-3 position, in which case the term 'gallate' is added to the name (Fig. 1.2). These latter catechins are referred to as 'galloylated catechins'.22-24 The group of catechins comprise four structurally different compounds (Fig. 1.2), plus their four epimers, of which catechin gallate $(\mathrm{Cg})$ and gallocatechin gallate $(\mathrm{GCg})$ hardly occur in tea leaves. Table 1.1 summarizes the content of the different catechins and their epimers in fresh tea leaves.
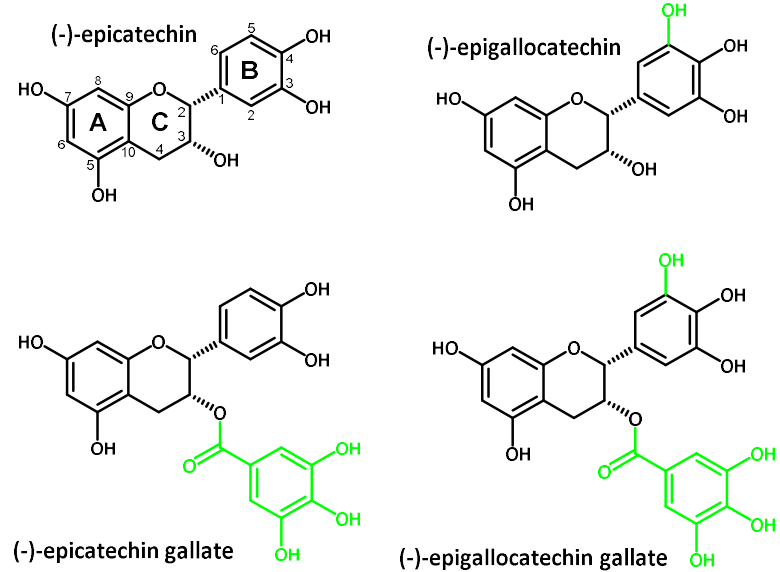

Figure 1.2 The four types of endogenous tea catechins, their differences are highlighted in green. 
As stated above, catechins are the main substrates in the enzymatic browning process of tea. During the oxidation reactions, catechins are transformed into a range of products referred to as the theaflavins (TFs) and the thearubigins (TRs). ${ }^{25}$ These classes of compounds are distinguished from each other by their solubility in ethyl acetate, although it is unclear how strict this division is for the various members of TRs. Quite a number of products belonging to the TRs have been identified, including their reaction pathways. ${ }^{2,4}$ Figure 1.3 schematically shows the reaction pathways to the main oxidation products present in black tea, which are the TFs, ${ }^{26,27}$ followed by theasinensins (TSs), ${ }^{28-31}$ theacitrins $(\mathrm{TCs})^{32,33}$ and theanaphtoquinones (TNQs). ${ }^{34}$ They differ in the types of catechins that reacted (Fig 1.3), and the resulting interflavanic configurations. An interflavanic configuration refers to the cross-link between two subunits, including the structural changes that occurred within the molecules themselves as a result of the coupling reaction.

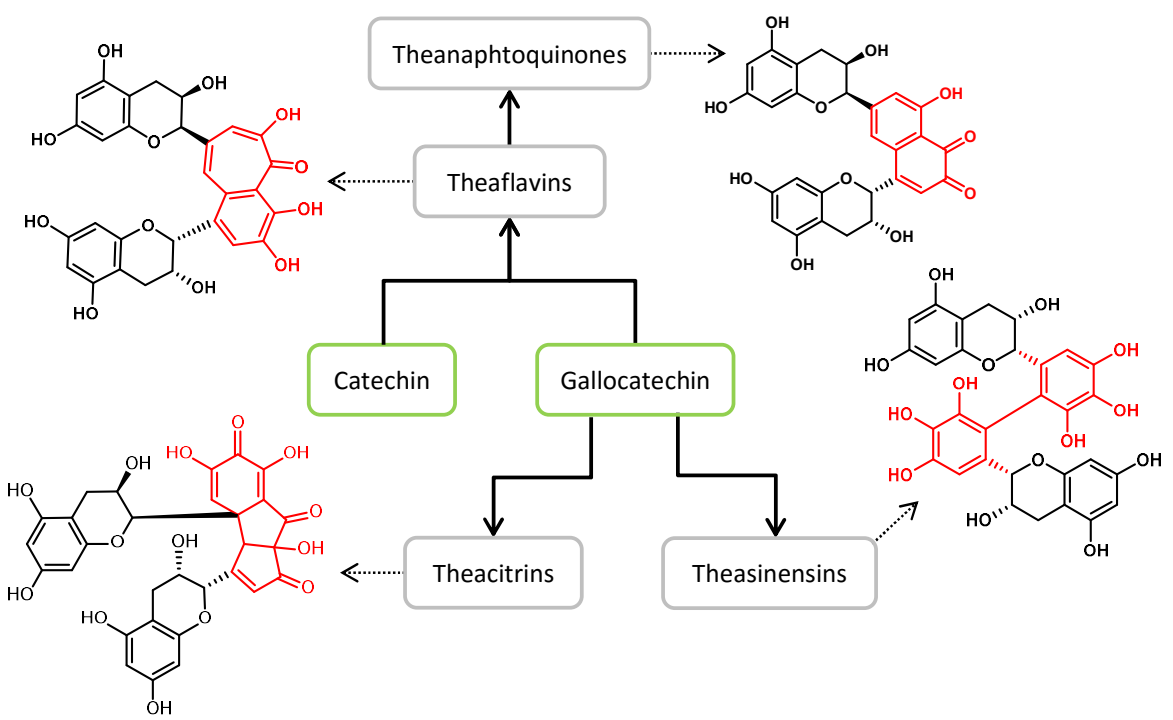

Figure 1.3 Schematic overview of the reaction pathways to the major oxidation projects from catechins. The characteristic interflavanic configurations are highlighted in red. Adapted from $2,27,30,31,33,34$.

TFs and TNQs are both formed from a catechin and a gallocatechin. TFs are characterized by their seven-membered benzotropolone ring, TNQs are oxidation products of TFs, and contain an $o$-quinone as part of their interflavanic configuration. TSs are formed from two gallocatechins that are both oxidized into an o-quinone. Subsequently, their B-rings are coupled via a C-C bond. TCs are also formed from two gallocatechins, only one of which has been oxidized prior to coupling. The interflavanic configuration of TCs have three fused rings, two of which are five-membered rings. 
The catechins used to form the oxidation products (Fig 1.3) can also be galloylated, resulting in the mono- or digalloylated versions of the oxidation products. ${ }^{2}$ The galloyl groups themselves can also participate in oxidation reactions, resulting for instance in theaflavates. ${ }^{2}$ Even though the gallate group is not necessary in the formation of TCs, solely galloylated TCs have been discovered in tea. ${ }^{32}$ It has been suggested that a C-3 galloylgroup is needed to stabilize the interflavanic configuration of TCs by a stacking interaction. ${ }^{33}$

The nomenclature used for the oxidation products of tea catechins is quite trivial, with no logical connection to the constituent catechin subunits or to the interflavanic configurations involved.

\subsection{Oxidative cascade hypothesis}

The four oxidation products from black tea described in Figure 1.3 are only the tip of the iceberg of about 10,000 different compounds suggested to be present in a cup of black tea. ${ }^{I}$ Several hypotheses about the formation of all these different compounds have been put forward throughout the years, ${ }^{35-38}$ with the 'oxidative cascade hypothesis' ${ }^{l}$ as the last and most complete description. The word 'cascade' in the name refers mainly to the hydroxylation level (level 2), where every hydroxylation will increase the probability of a next hydroxylation. The different levels within the 'oxidative cascade hypothesis' are schematically shown in Figure 1.4. With each level, the number of unique oxidation products increases.

The 'oxidative cascade hypothesis' starts with the native catechin building blocks that can connect via four different types of interflavanic configurations: the TS-type, the TFtype, the TNQ-type and the TC-type (Fig. 1.3). Level 1 of the oxidative cascade is the oligomerization of the catechin subunits into oligomers up to maximum DP 7, via these four types of configuration. ${ }^{1,39}$

The second level in the hypothesis refers to the nucleophilic attacks of water on any oligomeric quinone, upon which a hydrogen attached to the aromatic ring is replaced by a hydroxyl group. ${ }^{40,41}$ With each hydroxylation regio-isomers will be formed.

In the third level described in the 'oxidative cascade hypothesis', the redox equilibrium of the polyhydroxylated oligomers with their quinone form is considered. Because the oligomers have extra hydroxyl groups attached, different quinones can be formed within the oligomer, and these quinone structures will be in equilibrium with each other. The presence of these quinones in black tea will increase the number of unique compounds as well. ${ }^{40}$

Finally, the fourth level refers to the derivatives of the polyhydroxylated oligomers that are methylated or glycosylated. So far, no unambiguous proof has been provided for the 
occurrence of this fourth level. It might be that glycation or $O$-methylation was already an intrinsic part of the catechin subunits. ${ }^{42}$ In this thesis, the fourth level will not be further discussed.

The first, second and third level of the 'oxidative cascade hypothesis' all involve oxidation reactions. It has not been specified though, which oxidative enzymes are responsible for the different levels or for the resulting different interflavanic configurations. Besides direct enzymatic oxidation, also coupled oxidation is a possibility, in which the enzymatic oxidation plays an indirect role. In coupled oxidation, electrons are transferred from an electron donor to an electron acceptor, without direct mediation by an enzyme. In tea, catechin (C) and epicatechin (EC) quinones (enzymatically oxidized) can act as electron acceptors. These oxidized products regenerate back into $\mathrm{C}$ and $\mathrm{EC}$ after taking up electrons from, for instance, epigallocatechin (EGC) or TFs, consequently oxidizing the latters. ${ }^{43-46}$ This electron transport is driven by the higher redox potentials of the simple catechins (C and EC) compared to those of gallocatechins and theaflavin (Table 1.3). ${ }^{9}$

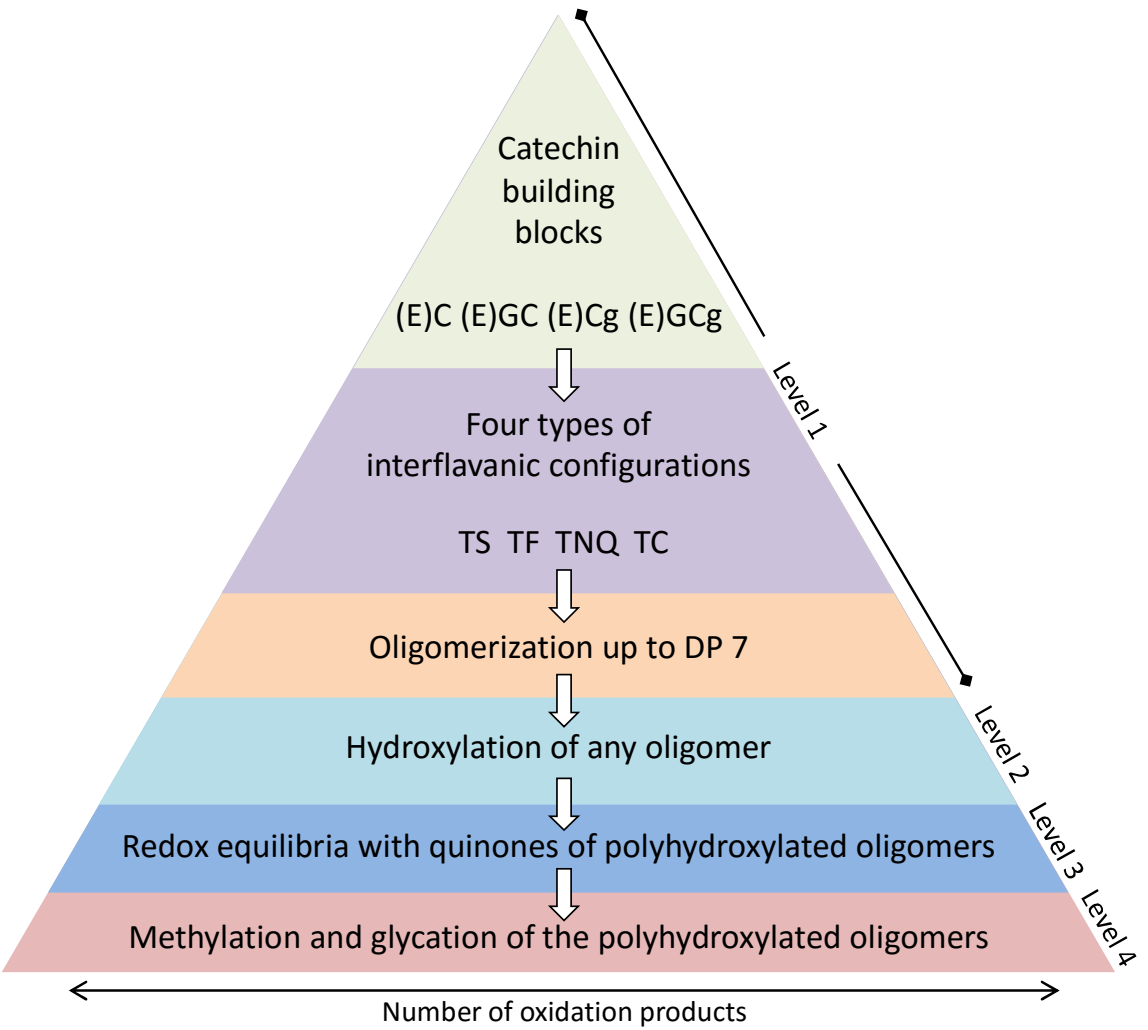

Figure 1.4 Schematic representation of the levels within the 'oxidative cascade hypothesis', and the increase in number of unique oxidation products with every step, adapted from ${ }^{1}$. 
Table 1.3 Redox potentials of catechins and theaflavins. ${ }^{9}$

\begin{tabular}{lc}
\hline Component & Redox potential $(\mathbf{V})^{*}$ \\
\hline Epigallocatechin (EGC) & 0.09 \\
\hline Gallocatechin (GC) & 0.13 \\
\hline Epigallocatechin gallate (EGCg) & 0.14 \\
Gallocatechin gallate (GCg) & 0.15 \\
Theaflavin (TF) & 0.16 \\
Theaflavin digallate (TFgg) & 0.19 \\
Epicatechin (EC) & 0.19 \\
Theaflavin gallate (TFg) & $0.19-0.20$ \\
Epicatechin gallate (ECg) & 0.20 \\
Catechin (C) & 0.20 \\
$*$ Determined at 20 ${ }^{\circ} \mathrm{C}$, at $\mathrm{pH}$ 6.15 at $1 \mathrm{mM}$ concentration in aqueous \\
solution, using a saturated calomel electrode (SCE) as reference.
\end{tabular}

\subsection{Key enzymes in tea fermentation}

Different types of oxidation reactions (oligomerization, hydroxylation) have been described by the 'oxidative cascade hypothesis', but the oxidative enzymes from tea leaves responsible for the different reactions are not exactly known yet. In tea leaves the oxidative enzymes polyphenol oxidase (PPO) and peroxidase (POD) are known to be present. ${ }^{47,48}$ POD is present in five times higher concentrations than PPO. ${ }^{49}$ Very little biochemical information on the tea oxidases has been reported, in contrast to the high importance of the enzymes for the formation of black tea.

POD is a heme-enzyme that can catalyse the oxidation of $o$-diphenols into $o$-quinones via a radical reaction (Fig 1.5). ${ }^{14,50,51}$ POD requires a peroxide as oxidizing agent to catalyse the oxidation reaction, for instance $\mathrm{H}_{2} \mathrm{O}_{2}$. The $\mathrm{pH}$ optima found for the different isozymes of POD from tea leaves are between $\mathrm{pH} 4.5$ and 5.0, ${ }^{50}$ at $\mathrm{pH} 6.0^{14}$ and at $\mathrm{pH} 7.0 .^{14}$

PPOs are copper containing enzymes ${ }^{13,52}$ that can be divided into two subclasses: (i) cresolase (or tyrosinase in animals) and (ii) catecholase. Cresolase activity includes both the hydroxylation of a mono-phenol into an $o$-diphenol and the oxidation of an $o$-diphenol into an $o$-quinone. Catecholase activity only catalyses the latter reaction (Fig. 1.5) ${ }^{53}$ PPOs need oxygen as oxidizing agent for catalysis. The $\mathrm{pH}$ optima for tea leaf PPOs have been determined to be $\mathrm{pH} 5.0^{13,53}$ or between $\mathrm{pH} 5.5$ and $5.8 . .^{54,55}$

It has been suggested that laccase is also part of the tea PPOs, because it also needs oxygen as oxidizing agent, ${ }^{52}$ and laccases are also able to catalyse the oxidation of $o$ diphenols into $o$-quinones. However, laccases form quinones via a radical reaction, ${ }^{53,56}$ therewith differentiating themselves from cresolase and catecholase activities (Fig 1.5). Laccases have much wider substrate specificity than only monophenols and/or $o$-diphenols. 
They can, for instance, oxidize $p$-diphenols, triphenols, and they even have activity towards lignin. ${ }^{57}$ So far, no unambiguous proof has been provided for the occurrence of laccases in tea leaves. However, their activity might have been incorporated in tea PPO activities, as often the kind of PPO activity is ill-defined. With few exceptions, ${ }^{52,54,58}$ in most literature reports the type of oxidative activity was not specified for tea leaf PPOs. ${ }^{16,49,59-63}$

PPOs are most active at the onset of tea oxidation. ${ }^{48}$ The $\mathrm{pH}$ of fresh tea leaves is between 5.2 and 5.8, which is around the optimum $\mathrm{pH}$ of PPOs. ${ }^{16,54,60}$ During the fermentation (oxidation) the $\mathrm{pH}$ decreases as a result of accumulation of acidic oxidation products $^{60}$ (e.g. theaflavic acids, proepitheaflagallin, and TRs after radical A-ring cleavage $^{2}$ ), which is assumed to be a reason for the decreasing activity of PPOs. The formation of TFs and TRs will also contribute to the inhibition of PPOs ${ }^{55}$ During the oxidation of catechins by $\mathrm{PPO}$, indirectly also $\mathrm{H}_{2} \mathrm{O}_{2}$ is produced, ${ }^{16,64,65}$ the reaction mechanism of which is still unknown. ${ }^{65,66}$

As tea fermentation progresses, PODs become more active during tea oxidation. ${ }^{48,62}$ This has two reasons: (i) the decreased $\mathrm{pH}$ is more optimal for POD, ${ }^{50}$ and (ii) $\mathrm{H}_{2} \mathrm{O}_{2}$ is being produced, which POD needs in order to catalyse oxidation reactions. This means that PPO and POD actually collaborate in the oxidation of tea phenolics.

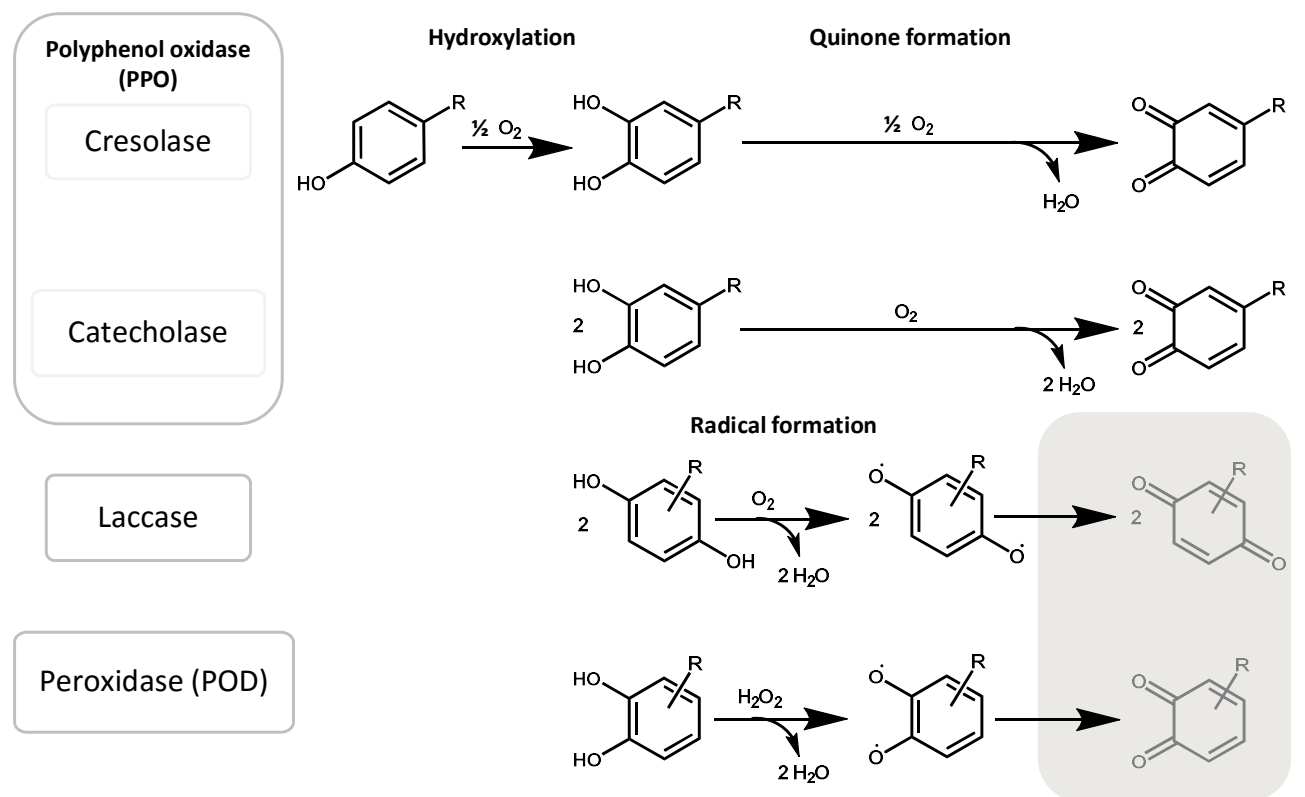

Figure 1.5 Schematic overview of the different oxidative enzyme activities in tea leaves. The quinone-formation step resulting from indirect enzymatic activity is shaded grey. 


\subsection{Black tea analysis until now}

It has been found already during early tea research that a black tea was too complicated to be analysed as such. Different fractionation methods have been established to be able to analyse the compounds in black tea. One of the first fractionations was based on partitioning in ethyl acetate. The ethyl acetate soluble compounds were later defined as the TFs, whereas the ethyl acetate insoluble compounds were defined as the TRs. ${ }^{25,67}$ Additional fractionation involved reversed-phase solid-phase-extraction with different concentrations of ethanol, ${ }^{68}$ or the complexation and precipitation of, mainly, TFs with caffeine. ${ }^{1,69,70}$ The latter is caused by self-association of TFs into clusters, and subsequent stacking of caffeine with the TF-clusters. ${ }^{71}$

Identification of the oxidation products of tea catechins started off with paper chromatography. ${ }^{25}$ TFs showed an orange-red band and TRs a brown band. Both TFs and TRs have wavelength maxima at 380 and $460 \mathrm{~nm}$. From separate TF and TR fractions their approximate concentration could be calculated by using these wavelengths. For TFs, correction factors were determined that took into account the difference in absorbance between the galloylated and non-galloylated TFs, whereas the galloylated TFs have a higher molar extinction coefficient. ${ }^{72}$ TRs are an undefined mixture of oxidation products, which was not constant enough for determination of such correction factors. For estimation of the amount of TRs, the ratio of the absorbances at the wavelength maxima $\left(\mathrm{E}_{380} / \mathrm{E}_{460}\right)$ was used to estimate the concentration. A high ratio indicated relatively light colour and low concentration of TRs, whereas a low ratio indicated a deep colour and higher concentration of TRs. ${ }^{67,73}$

When the different TFs were purified to completeness, the molar extinction coefficients of the TFs were determined (Table 1.4). ${ }^{72}$ In comparison to tea catechins, the larger conjugated system of TFs resulted in higher molar extinction coefficients. The maximum absorbance around $460 \mathrm{~nm}$ indicates their orange-red colour. Compounds from TR fractions have not been purified yet, and consequently, no information on the absorbance of individual molecules is available.

Identification procedures of compounds included high-performance liquid chromatography (HPLC) analysis, mostly combined with NMR. ${ }^{59,74}$ A phenomenon often found with liquid chromatography is the occurrence of a rise in the baseline, the so-called 'hump', in the chromatogram. This hump is caused by the large number of distinct compounds that vary very slightly in their structures and polarities. Therefore, they will elute very close to each other, thereby causing an undistinguishable 'hump' in the chromatogram. ${ }^{1,34,49,75}$ 
Table 1.4 Molar extinction coefficients* of different theaflavins and catechins at the wavelengtht of maximum absorbance.

\begin{tabular}{|c|c|c|c|c|}
\hline & $\lambda_{\max }\left(\varepsilon_{\max }\right)^{\ddagger}$ & $\lambda_{\max }\left(\varepsilon_{\max }\right)$ & $\lambda_{\max }\left(\varepsilon_{\max }\right)^{\ddagger}$ & $\lambda_{\max }\left(\varepsilon_{\max }\right)^{\ddagger}$ \\
\hline Theaflavin (TF) & $268(21,400)$ & $294(17,900)$ & $379(10,100)$ & $467(3,700)$ \\
\hline Theaflavin gallate (TFg) & $275(30,500)$ & & $378(9,150)$ & $462(3,300)$ \\
\hline Theaflavin digallate (TFgg) & $278(35,200)$ & & $378(9,200)$ & $460(3,160)$ \\
\hline Catechin (C) & $270(2,484)$ & & & \\
\hline Epicatechin (EC) & $270(4,290)$ & & & \\
\hline Epigallocatechin (EGC) & $270(2,088)$ & & & \\
\hline Gallocatechin gallate (GCg) & $270(11,009)$ & & & \\
\hline Epigallocatechin gallate (EGCg) & $270(11,920)$ & & & \\
\hline
\end{tabular}

Mass spectrometric (MS) measurements were added as extra identification of the compounds, and they were, for instance, used to elucidate structures after degradation by chemical treatment (e.g. with $\mathrm{KMnO}_{4}$ ). ${ }^{37}$ Single ion monitoring (SIM) settings were introduced in LC-MS analysis, in order to be able to only analyse a single specified ion during the run. This was used in tea to analyse compounds that were first only found in a model system ${ }^{77}$. When tandem MS became available, LC-MS/MS measurements were used to identify compounds by their fragmentation patterns. ${ }^{1,78}$ Still, the full potential of the MS is not used in tea analysis. It might be a good tool in identification of compounds present in low abundance, when using more specified settings during LC-MS than used until now. LC-MS can be used as a rapid identification tool, which only requires limited to no purification of compounds, in contrast to sample preparation for structure elucidation with NMR spectroscopy.

Matrix-assisted laser desorption/ionization time-of-flight mass spectrometry (MALDITOF-MS) measurements were used to try to further elucidate the TRs. ${ }^{36}$ MALDI-TOF-MS can measure higher $\mathrm{m} / \mathrm{z}$ values than LC-MS, and because there is no chromatographic separation of the compounds, all isomers of one compound are collectively represented by one peak. In this way, higher $\mathrm{Mw}$ isomers, normally scattered over the chromatogram in too low abundance to be identified, are now made visible in one peak. Recently, ultra-high resolution mass spectra could be acquired, with Fourier-transform ion-cyclotron-resonance mass spectrometry (FT-ICR-MS). With the exact mass obtained in this technique, molecular formulas of compounds were determined. Combined with element analysis software the number of compounds in a black tea was estimated (almost 10,000). ${ }^{1,75}$

In analysis of phenolics present in tea, it was shown that a combination of analytical techniques gives the best result. ${ }^{75}$ Instead of fractionation of black tea, model systems have also been used for analysis of oxidation products. These models often used PPO extracts 
from tea leaves or other origin, not specifying the kind of oxidative activity, or used chemical oxidation of compounds. ${ }^{79}$

The specific oxidative activities responsible for the formation of these compounds have hardly been investigated. In this thesis, model systems will be used to characterize the potential contribution of the different enzymes to the phenolics profile of black tea. Instead of using a PPO extract from tea leaves, possibly containing multiple enzyme activities, distinct activities will be employed by making use of exogenous enzymes, to specifically link product formation to particular oxidative activities.

\subsection{Sensory aspects of tea}

The sensory aspects of a tea are very important. They include taste, mouthfeel, appearance (colour and tea creaming), and aroma. Tea is well-known for its typical bitter taste, green or brown colour, astringent mouthfeel and typical aroma. These parameters are used when professional tea tasters assess the quality of teas. ${ }^{6}$ Besides those parameters, also the tea creaming effect, i.e. the appearance of a haze when the tea cools down, is an important quality parameter used by tea tasters, merely as an aid to visually determine the intensity of a tea. Table $\mathbf{1 . 5}$ gives an overview of the tea compounds responsible for the different sensory characteristics, except for aroma, which is not discussed in this thesis, because the focus was on non-volatile compounds. The specific contribution to sensory aspects of tea by TRs is hardly known. Obtaining pure compounds from the thearubigins group would be beneficial for sensory analysis, but given their complexity and number of representatives, this almost seems a mission impossible.

\subsubsection{Colour}

The colour of tea is always between light yellowish-green for green teas to red-brown for black teas. In green tea, mainly catechins are present, which are colourless. ${ }^{9}$ The small amount of chlorophyll that is extracted, is responsible for the green colour. Chlorophyll is a lipophilic molecule, so only small amounts of the colour are extracted, resulting in a light green shade. ${ }^{4,80}$ In black tea, the red-brown TRs and red-orange TFs are responsible for the colour. ${ }^{81}$ It was found that the TFs are more intensely coloured and contribute more to the colour of a black tea than the TRs. This was shown by a 3 to 8 times higher absorbance at $460 \mathrm{~nm}$ (red-orange) of TF fractions compared to TR fractions, ${ }^{67}$ although this does not seem to be in accordance with the 30 times higher TR content compared to TFs. Nevertheless, taste evaluations showed a positive correlation of tea quality with TF contents. ${ }^{67,82,83}$ During the enzymatic oxidation, also senescence of the tea leaves occurs, which causes chlorophyll to degrade into pheophytins and pheophorbides. These molecules 
provide the characteristic black-brown colour to the leaves, and will be partly extracted in the tea as well. ${ }^{4,80}$

\subsubsection{Taste}

The bitter taste of tea was found to be mainly caused by caffeine ${ }^{21}$ and catechins. ${ }^{84,85}$ Therefore, green teas tend to be more bitter than black teas. The bitter effect of caffeine was shown to decrease in the presence of TFs, ${ }^{21}$ due to the complexation of TFs with caffeine. ${ }^{71}$ In contrast, flavonol-glycosides were shown to have an amplifying effect on the bitterness of caffeine, no explanation for this phenomenon was provided. ${ }^{12}$ These findings were based on sensory studies.

In bitterness studies using human taste receptors, catechins and theaflavins were found to bind to hTAS2R14 and hTAS2R39. ${ }^{84,86,87}$ The effective concentrations at half maximum response $\left(\mathrm{EC}_{50}\right.$ : a lower $\mathrm{EC}_{50}$ represents higher bitterness) of the TFs were in general lower than those of the non-oxidized catechins. On the hTAS2R39 receptor, TF, TFg, and TFgg, had $\mathrm{EC}_{50} \mathrm{~s}$ of $2.79,0.67$, and $1.55 \mu \mathrm{M}$, respectively, ${ }^{86}$ whereas $\mathrm{ECg}, \mathrm{EGCg}, \mathrm{EGC}$, and EC had $\mathrm{EC}_{50}$ 's of $88.2,181.6,395.5$, and $417.7 \mu \mathrm{M}$, respectively. ${ }^{84}$ Especially for catechins, increased bitterness correlated with the presence of a galloyl-group (compare ECg and EGCg with EGC and EC). ${ }^{84}$ The more sensitive response of bitter receptors to TFs than to catechins is in contradiction with the results of the sensory studies. This might be explained by complex formation between TF and caffeine ${ }^{21,88}$ or proteins, ${ }^{11,89}$ resulting in a higher threshold for bitterness perception in vivo than in vitro.

No information on the contribution to bitterness for oxidation products of tea phenolics other than TF (e.g. thearubigins) is known yet. This is most likely because of the difficulty in purifying these compounds, either by fractionating them from mixtures obtained with model systems or by fractionating them directly from tea.

Free amino acids in tea, mainly theanines, add to the umami taste, which is specifically present in Japanese green teas. ${ }^{9,90}$

\subsubsection{Mouth feel}

The most important factor relating to mouth feel is astringency, especially in black tea. Astringency is commonly perceived as the dry, puckering sensation in the mouth. It is thought to be caused by an interaction between the tea phenolics and proteins from the saliva. $^{91}$ The proteins normally interact with the oral mucosal cells to form a lubricant soft layer in the mouth. When phenolic compounds interact with these proteins, this obstructs the interaction with the mucosal cells, diminishing lubrication. Besides, the proteinphenolic complexes become insoluble, resulting in extra friction in the mouth. ${ }^{91-93}$ 
The protein-phenolic interaction is described in a three-stage-model. The first stage is the binding of random coil protein to polyphenols, resulting in soluble complexes. This binding occurs via hydrophobic interactions $(\mathrm{CH}-\pi$ stacking) between the planar aromatic rings of the phenolics to the pyrrolidine ring of a proline amino acid in the protein. Simultaneously, this interaction is reinforced by hydrogen bonding between hydroxyl groups on the phenolics and the carbonyl groups of the protein. The second stage is when the polyphenol concentration rises and cross-links are formed between the proteinpolyphenol complexes, resulting in insoluble protein dimers. The final stage is the aggregation of the dimers, and precipitation of the large particles. ${ }^{92}$

Different phenolics have different contributions to the astringency. The main properties of phenolics that play a role in protein binding are: (i) the degree of polymerization, ${ }^{94-96}$ (ii) the conformational flexibility, ${ }^{96}$ (iii) the degree of hydroxylation (DP) ${ }^{94,95}$ (iv) the hydrophobicity, ${ }^{96}$ and (v) the galloyation. ${ }^{94}$ An increase in either of these properties will result in an increased astringent mouth feel, except for DP, which shows an optimum. After the optimum, the binding affinity decreases due to steric hindrance. ${ }^{97}$

The positive correlation of TF content with tea quality is also related to this typical astringent mouth feel, ${ }^{47}$ especially for galloylated $\mathrm{TFs} .{ }^{98,99}$ By adding milk to the tea, astringency is decreased, because the TFs bind to the milk proteins and are not available to bind with the saliva proteins anymore. ${ }^{89}$ Furthermore, it was found that the astringency of TFs was reduced after complexation with caffeine. ${ }^{2 l}$ Finally, flavonol glycosides appear to contribute considerably to astringency, with on average 10 to 100 times lower taste thresholds for astringency than TFs. ${ }^{11}$ However, flavonol glycosides are known to have poor affinity for proteins, hence, another mechanism than phenolic-protein interaction will also play a role in astringency. ${ }^{100}$

\subsubsection{Tea creaming}

Tea creaming is a phenomenon occurring when cooling down tea below $60{ }^{\circ} \mathrm{C} \cdot{ }^{70,71}$ It is caused by the complexation of caffeine with phenol compounds, ${ }^{69}$ mainly TFs, in the presence of calcium ions. ${ }^{88,101}$ It results in a red brown haze in a black tea. TFs were shown to form non-covalently bound dimers, by self-association of the two planar benzotropolone moieties. For both TFs, the two benzopyran moieties, orthogonal of to the benzotropolone planes, engage in a stacking interaction. The caffeine molecules can subsequently interact, in a sequential manner, with the four benzopyran moieties to stabilize the cluster. ${ }^{71,88}$ 
Table 1.5 Overview of the contribution of phenolic compounds, and theanine to the sensory characteristics of tea.

\begin{tabular}{|c|c|c|c|c|}
\hline & Colour & Taste & Mouth feel & Creaming \\
\hline Catechins & colourless $^{9}$ & bitterness $^{84,85}$ & n.a. & n.a. \\
\hline Theaflavins & red/orange $\mathrm{e}^{25,67}$ & $\begin{array}{l}\text { decreases bitterness } \\
\text { of caffeine }{ }^{2 I}\end{array}$ & astringency ${ }^{91,93}$ & Tea creaming ${ }^{69,70}$ \\
\hline Thearubigins & $\mathrm{red} /$ brown $^{25,67}$ & n.a. & 'body' of the tea ${ }^{102}$ & n.a. \\
\hline $\begin{array}{l}\text { Flavonol- } \\
\text { glycosides }\end{array}$ & colourless $^{11}$ & $\begin{array}{l}\text { amplify bitterness of } \\
\text { caffeine }\end{array}$ & astringency $^{11}$ & n.a. \\
\hline Caffeine & colourless $^{103}$ & bitterness $^{2 I}$ & $\begin{array}{l}\text { decreases } \\
\text { astringency of } \\
\text { theaflavins }\end{array}$ & Tea creaming ${ }^{69,70}$ \\
\hline Theanine & colourless $^{104}$ & umami $^{9,90}$ & n.a. & n.a. \\
\hline Chlorophyll & $\begin{array}{l}\text { green (during } \\
\text { senescence it turns } \\
\text { in brown/black } \\
\text { pheophytins and } \\
\text { pheophorbides) }\end{array}$ & n.a. & n.a. & n.a. \\
\hline
\end{tabular}

\subsection{Aim and outline of the thesis}

From the above it is clear that the oxidation process occurring in green tea leaves during black tea manufacturing is an uncontrolled process. The resulting oxidation products, however, are very important for the quality of the tea. Subtle differences in the phenolic profile can lead to changes in the sensory characteristics of a tea. Therefore, more control of the oxidation process would be desirable. By using a model system with specific tea catechins and oxidative enzyme activities, detailed knowledge of the oxidation process can be obtained. With the objective to turn black tea oxidation into a more controlled process in the long run, the aim of this thesis is to (i) obtain understanding of the enzymatic oxidation reactions, described in the 'oxidative cascade hypothesis', occurring in tea oxidation, and (ii) enable more rapid analysis of complex mixtures of phenolics without prior preparative fractionation steps.

In Chapter 2 the focus is on formation of TFs from tea catechins. A model system is used to enable identification of the distinct contribution of cresolase (tyrosinase) and laccase activity to the formation and stability of TFs. Further oxidation of TF occurred, and these subsequent oxidation products of TF are described in Chapter 3. The formation of these two-step-oxidation products is shown in a model system. A rapid screening method based on UHPLC-MS was developed to identify these low abundance compounds in a 
complex mixture as tea, without the need of prior fractionation of the sample. In Chapter 4 this rapid screening method is used as well, to show the presence of oxidation products from only (epi)catechins, i.e. dehydrocatechins, in black tea, after they had been identified from a model system. The role of POD activity in the hydroxylation step of the 'oxidative cascade hypothesis' is investigated in Chapter 5. Finally, Chapter 6 addresses the prospects and limitations of the use of model systems and the rapid screening method developed. With the knowledge on the oxidative enzymes and oxidation products obtained in the former chapters, a generic nomenclature for catechin oxidation products is proposed, and potential design options for tea manufacturing are elaborated. The effect of the different oxidation levels in the 'oxidative cascade hypothesis' on sensory characteristics are discussed as well.

\subsection{References}

1. Kuhnert, N.; Drynan, J. W.; Obuchowicz, J.; Clifford, M. N.; Witt, M. Mass spectrometric characterization of black tea thearubigins leading to an oxidative cascade hypothesis for thearubigin formation. Rapid Communications in Mass Spectrometry 2010, 24, 3387-3404.

2. Drynan, J. W.; Clifford, M. N.; Obuchowicz, J.; Kuhnert, N. The chemistry of low molecular weight black tea polyphenols. Natural Product Reports 2010, 27, 417-462.

3. Wright, L. P.; Mphangwe, N. I. K.; Nyirenda, H. E.; Apostolides, Z. Analysis of caffeine and flavan-3-ol composition in the fresh leaf of Camellia sinesis for predicting the quality of the black tea produced in Central and Southern Africa. Journal of the Science of Food and Agriculture 2000, 80, 1823-1830.

4. Harbowy, M. E.; Balentine, D. A. Tea chemistry. Critical Reviews in Plant Sciences 1997, 16, 415-480.

5. $\quad$ Lin, Y. L.; Juan, I. M.; Chen, Y. L.; Liang, Y. C.; Lin, J. K. Composition of polyphenols in fresh tea leaves and associations of their oxygen-radical-absorbing capacity with antiproliferative actions in fibroblast cells. Journal of Agricultural and Food Chemistry 1996, 44, 1387-1394.

6. Vossen, H. A. M. v. d.; Wessel, M., Eds. Plant resources of South-East Asia No 16. Stimulants; Backhuys Publishers: Leiden, The Netherlands, 2000.

7. Obanda, M.; Owuor, P. O.; Taylor, S. J. Flavanol composition and caffeine content of green leaf as quality potential indicators of Kenyan black teas. Journal of the Science of Food and Agriculture 1997, 74, 209215.

8. Li, S.; Lo, C. Y.; Pan, M. H.; Lai, C. S.; Ho, C. T. Black tea: Chemical analysis and stability. Food and Function 2013, 4, 10-18.

9. Balentine, D. A.; Wiseman, S. A.; Bouwens, L. C. M. The chemistry of tea flavonoids. Critical Reviews in Food Science and Nutrition 1997, 37, 693-704.

10. Graham, H. N. Green tea composition, consumption, and polyphenol chemistry. Preventive Medicine 1992, 21, 334-350.

11. Scharbert, S.; Holzmann, N.; Hofmann, T. Identification of the astringent taste compounds in black tea infusions by combining instrumental analysis and human bioresponse. Journal of Agricultural and Food Chemistry 2004, 52, 3498-3508. 
12. Scharbert, S.; Hofmann, T. Molecular definition of black tea taste by means of quantitative studies, taste reconstitution, and omission experiments. Journal of Agricultural and Food Chemistry 2005, 53, 53775384

13. Halder, J.; Tamuli, P.; Bhaduri, A. N. Isolation and characterization of polyphenol oxidase from Indian tea leaf (Camellia sinensis). Journal of Nutritional Biochemistry 1998, 9, 75-80.

14. Chen, G. X.; Asada, K. Ascorbate peroxidase in tea leaves: Occurrence of two isozymes and the differences in their enzymatic and molecular properties. Plant and Cell Physiology 1989, 30, 987-998.

15. Suzuki, T.; Yamazaki, N.; Sada, Y.; Oguni, I.; Moriyasu, Y. Tissue distribution and intracellular localization of catechins in tea leaves. Bioscience, Biotechnology and Biochemistry 2003, 67, 2683-2686.

16. Subramanian, N.; Venkatesh, P.; Ganguli, S.; Sinkar, V. P. Role of polyphenol oxidase and peroxidase in the generation of black tea theaflavins. Journal of Agricultural and Food Chemistry 1999, 47, 2571-2578.

17. Tomlins, K. I.; Mashingaidze, A. Influence of withering, including leaf handling, on the manufacturing and quality of black teas - A review. Food Chemistry 1997, 60, 573-580.

18. Tanaka, T.; Miyata, Y.; Tamaya, K.; Kusano, R.; Matsuo, Y.; Tamaru, S.; Tanaka, K.; Matsui, T.; Maeda M.; Kouno, I. Increase of theaflavin gallates and thearubigins by acceleration of catechin oxidation in a new fermented tea product obtained by the tea-rolling processing of loquat (Eriobotrya Japonica) and green tea leaves. Journal of Agricultural and Food Chemistry 2009, 57, 5816-5822.

19. Karori, S. M.; Wachira, F. N.; Wanyoko, J. K.; Ngure, R. M. Antioxidant capacity of different types of tea products. African Journal of Biotechnology 2007, 6, 2287-2296.

20. Hilal, Y.; Engelhardt, U. Characterisation of white tea - comparison to green and black tea. Journal of Consumer Protection and Food Safety 2007, 2, 414-421.

21. Millin, D. J.; Crispin, D. J.; Swaine, D. Nonvolatile components of black tea and their contribution to the character of the beverage. Journal of Agricultural and Food Chemistry 1969, 17, 717-722.

22. Bradfield, A. E.; Bate-Smith, E. C. Chromatographic behaviour and chemical structure II. The tea catechins. Biochimica et Biophysica Acta 1950, 4, 441-444.

23. Bradfield, A. E.; Penney, M.; Wright, W. B. The catechins of green tea. Part I. Journal of the Chemical Society 1947, 32-36.

24. Bradfield, A. E.; Penney, M. The catechins of green tea. Part II. Journal of the Chemical Society 1948, 2249-2254.

25. Roberts, E. A. H.; Cartwright, R. A.; Oldschool, M. The phenolic substances of manufactured tea. I. Fractionation and paper chromatography of water-soluble substances. Journal of Agricultural and Food Chemistry 1957, 8, 72-80.

26. Takino, Y.; Ferretti, A.; Flanagan, V.; Gianturco, M.; Vogel, M. The structure of theaflavin, A polyphenol of black tea. Tetrahedron Letters 1965, 6, 4019-4025.

27. Takino, Y.; Imagawa, H.; Harikawa, H.; Tanaka, A. Studies on the mechanism of the oxidation of the catechins. Part III, Formation of a reddish-orange pigment, its spectral relationship to some benzotropolone derivatives. Agriculture, Biology and Chemistry 1964, 28, 64-71.

28. Nonaka, G.; Kawahara, O.; Nishioka, I. Tannins and related compounds. XV. A new class of dimeric flavan-3-ol gallates, theasinensins A and B, and proanthocyanidin gallates from green tea leaf. Chemical \& Pharmaceutical Bulletin 1983, 31, 3906-3914.

29. Hashimoto, F.; Nonaka, G.; Nishioka, I. Tannins and related compounds. LXIX. Isolation and structure elucidation of B,B'-linked bisflavanoids, theasinensins D-G and oolongtheanin from oolong tea. (2). Chemical \& Pharmaceutical Bulletin 1988, 36, 1676-1684.

30. Tanaka, T.; Mine, C.; Watarumi, S.; Fujioka, T.; Mihashi, K.; Zhang, Y. J.; Kouno, I. Accumulation of epigallocatechin quinone dimers during tea fermentation and formation of theasinensins. Journal of Natural Products 2002, 65, 1582-1587. 
31. Matsuo, Y.; Tanaka, T.; Kouno, I. A new mechanism for oxidation of epigallocatechin and production of benzotropolone pigments. Tetrahedron 2006, 62, 4774-4783.

32. Davis, A. L.; Lewis, J. R.; Cai, Y.; Powell, C.; Davis, A. P.; Wilkins, J. P. G.; Pudney, P.; Clifford, M. N. A polyphenolic pigment from black tea. Phytochemistry 1997, 46, 1397-1402.

33. Matsuo, Y.; Li, Y.; Watarumi, S.; Tanaka, T.; Kouno, I. Production and degradation mechanism of theacitrin $\mathrm{C}$, a black tea pigment derived from epigallocatechin-3-O-gallate via a bicyclo[3.2.1]octane-type intermediate. Tetrahedron 2011, 67, 2051-2059.

34. Tanaka, T.; Betsumiya, Y.; Mine, C.; Kouno, I. Theanaphthoquinone, a novel pigment oxidatively derived from theaflavin during tea-fermentation. Chemical Communications 2000, 1365-1366.

35. Brown, A. G.; Eyton, W. B.; Holmes, A.; Ollis, W. D. The identification of the thearubigins as polymeric proanthocyanidins. Phytochemistry 1969, 8, 2333-2340.

36. Menet, M. C.; Sang, S.; Yang, C. S.; Ho, C. T.; Rosen, R. T. Analysis of theaflavins and thearubigins from black tea extract by MALDI-TOF mass spectrometry. Journal of Agricultural and Food Chemistry 2004, 52, 2455-2461.

37. Ozawa, T.; Kataoka, M.; Morikawa, K.; Negishi, O. Elucidation of the partial structure of polymeric thearubigins from black tea by chemical degradation. Bioscience, Biotechnology and Biochemistry 1996, 60, 2023-2027.

38. Haslam, E. Thoughts on thearubigins. Phytochemistry 2003, 64, 61-73.

39. Kuhnert, N. Unraveling the structure of the black tea thearubigins. Archives of Biochemistry and Biophysics 2010, 501, 37-51.

40. Kuhnert, N.; Clifford, M. N.; Müller, A. Oxidative cascade reactions yielding polyhydroxy-theaflavins and theacitrins in the formation of black tea thearubigins: Evidence by tandem LC-MS. Food and Function 2010, 1, 180-199.

41. Yassin, G. H.; Koek, J. H.; Jayaraman, S.; Kuhnert, N. Identification of novel homologous series of polyhydroxylated theasinensins and theanaphthoquinones in the SII fraction of black tea thearubigins using ESI/HPLC tandem mass spectrometry. Journal of Agricultural and Food Chemistry 2014, 62, 9848-9859.

42. Chiu, F. L.; Lin, J. K. HPLC analysis of naturally occurring methylated catechins, 3"- and 4"-methylepigallocatechin gallate, in various fresh tea leaves and commercial teas and their potent inhibitory effects on inducible nitric oxide synthase in macrophages. Journal of Agricultural and Food Chemistry 2005, 53, 7035-7042.

43. Bajaj, K. L.; Anan, T.; Tsushida, T.; Ikegaya, K. Effects of (-)-epicatechin on oxidation of theaflavins by polyphenol oxidase from tea leaves. Agricultural and Biological Chemistry 1987, 51, 1767-1772.

44. Kusano, R.; Tanaka, T.; Matsuo, Y.; Kouno, I. Structures of epicatechin gallate trimer and tetramer produced by enzymatic oxidation. Chemical and Pharmaceutical Bulletin 2007, 55, 1768-1772.

45. Tanaka, T.; Mine, C.; Inoue, K.; Matsuda, M.; Kouno, I. Synthesis of theaflavin from epicatechin and epigallocatechin by plant homogenates and role of epicatechin quinone in the synthesis and degradation of theaflavin. Journal of Agricultural and Food Chemistry 2002, 50, 2142-2148.

46. Li, Y.; Shibahara, A.; Matsuo, Y.; Tanaka, T.; Kouno, I. Reaction of the black tea pigment theaflavin during enzymatic oxidation of tea catechins. Journal of Natural Products 2010, 73, 33-39.

47. Sabhapondit, S.; Bhattacharyya, P.; Bhuyan, L. P.; Hazarika, M.; Goswami, B. C. Optimisation of withered leaf moisture during the manufacture of black tea based upon theaflavins fractions. International Journal of Food Science and Technology 2014, 49, 205-209.

48. Mahanta, P. K.; Boruah, S. K.; Boruah, H. K.; Kalita, J. N. Changes of polyphenol oxidase and peroxidase activities pigment composition of some manufactured black teas (Camellia, sinensis L.). Journal of Agricultural and Food Chemistry 1993, 41, 272-276. 
49. Finger, A. In-vitro studies on the effect of polyphenol oxidase and peroxidase on the formation of polyphenolic black tea constituents. Journal of the Science of Food and Agriculture 1994, 66, 293-305.

50. Kvaratskhelia, M.; Winkel, C.; Thorneley, R. N. F. Purification and characterization of a novel class III peroxidase isoenzyme from tea leaves. Plant Physiology 1997, 114, 1237-1245.

51. Nicell, J. A.; Wright, H. A model of peroxidase activity with inhibition by hydrogen peroxide. Enzyme and Microbial Technology 1997, 21, 302-310.

52. Gregory, R. P. F.; Bendall, D. S. The purification and some properties of the polyphenol oxidase from tea (Camellia sinensis L.). Biochemical Journal 1966, 101, 569-581.

53. Aniszewski, T.; Lieberei, R.; Gulewicz, K. Research on catecholases, laccases and cresolases in plants. Recent progress and future needs. Acta Biologica Cracoviensia Series Botanica 2008, 50, 7-18.

54. Coggon, P.; Moss, G. A.; Sanderson, G. W. Tea catechol oxidase: Isolation, purification and kinetic characterization. Phytochemistry 1973, 12, 1947-1955.

55. Pruidze, G. N.; McHedlishvili, N. I.; Omiadze, N. T.; Gulua, L. K.; Pruidze, N. G. Multiple forms of phenol oxidase from Kolkhida tea leaves (Camelia sinensis L.) and Mycelia sterilia IBR 35219/2 and their role in tea production. Food Research International 2003, 36, 587-595.

56. Osman, A. M.; Wong, K. K. Y.; Fernyhough, A. The laccase/ABTS system oxidizes (+)-catechin to oligomeric products. Enzyme and Microbial Technology 2007, 40, 1272-1279.

57. Mayer, A. M.; Staples, R. C. Laccase: New functions for an old enzyme. Phytochemistry 2002, 60, $551-565$.

58. Singh, D. V.; Mukherjee, P. P. Tyrosinase: A Fe ${ }^{2+}$ containing enzyme from tea leaves. Current Science 1973, 42, 391

59. Hashimoto, F.; Nonaka, G.; Nishioka, I. Tannins and related compounds. CXIV. Structures of novel fermentation products, theogalinin, theaflavonin and desgalloyl theaflavonin from black tea, and changes of tea leaf polyphenols during fermentation. Chemical and Pharmaceutical Bulletin 1992, 40, 1383-1389.

60. Cloughley, J. B.; Ellis, R. T. The effect of $\mathrm{pH}$ modification during fermentation on the quality parameters of central African black teas. Journal of the Science of Food and Agriculture 1980, 31, 924-934.

61. Hilton, P. J. In vitro oxidation of flavanols from tea leaf. Phytochemistry 1972, 11, 1243-1248.

62. Dix, M. A.; Fairley, C. J.; Millin, D. J.; Swaine, D. Fermentation of tea in aqueous suspension. Influence of tea peroxidase. Journal of the Science of Food and Agriculture 1981, 32, 920-932.

63. Stodt, U. W.; Blauth, N.; Niemann, S.; Stark, J.; Pawar, V.; Jayaraman, S.; Koek, J.; Engelhardt, U. H. Investigation of processes in black tea manufacture through model fermentation (oxidation) experiments. Journal of Agricultural and Food Chemistry 2014, 62, 7854-7861.

64. Richard-Forget, F. C.; Gauillard, F. A. Oxidation of chlorogenic acid, catechins, and 4-methylcatechol in model solutions by combinations of pear (Pyrus communis cv. williams) polyphenol oxidase and peroxidase: A possible involvement of peroxidase in enzymatic browning. Journal of Agricultural and Food Chemistry 1997, 45, 2472-2476.

65. Jiang, Y.; Miles, P. W. Generation of $\mathrm{H}_{2} \mathrm{O}_{2}$ during enzymic oxidation of catechin. Phytochemistry 1993, 33 , 29-34.

66. Nakayama, T.; Ichiba, M.; Kuwabara, M.; Kajiya, K.; Kumazawa, S. Mechanisms and structural specificity of hydrogen peroxide formation during oxidation of catechins. Food Science and Technology Research 2002, 8, 261-267.

67. Roberts, E. A. H.; Smith, R. F. Spectrophotometric measurements of theaflavins and thearubigins in black tea liquors in assessments of quality in teas. The Analyst 1961, 86, 94-98.

68. Rana, A.; Singh, H. P. A rapid HPLC-DAD method for analysis of theaflavins using C12 as stationary phase. Journal of Liquid Chromatography and Related Technologies 2012, 35, 2272-2279. 
69. Roberts, E. A. H. The phenolic substances of manufactured tea. X. The creaming down of tea liquors. Journal of the Science of Food and Agriculture 1963, 14, 700-705.

70. Powell, C.; Clifford, M. N.; Opie, S. C.; Ford, M. A.; Robertson, A.; Gibson, C. L. Tea cream formation: The contribution of black tea phenolic pigments determined by HPLC. Journal of the Science of Food and Agriculture 1993, 63, 77-86.

71. Charlton, A. J.; Davis, A. L.; Jones, D. P.; Lewis, J. R.; Davies, A. P.; Haslam, E.; Williamson, M. P. The self-association of the black tea polyphenol theaflavin and its complexation with caffeine. Journal of the Chemical Society, Perkin Transactions 2 2000, 2, 317-322.

72. Coxon, D. T.; Holmes, A.; Ollis, W. D.; Vora, V. C. The constitution and configuration of the theaflavin pigments of black tea. Tetrahedron Letters 1970, 11, 5237-5240.

73. Roberts, E. A. H.; Smith, R. F. The phenolic substances of manufactured tea. IX. The spectrophotometric evaluation of tea liquors. Journal of the Science of Food and Agriculture 1963, 14, 689-700.

74. Opie, S. C.; Robertson, A.; Clifford, M. N. Black tea thearubigins - their HPLC separation and preparation during in-vitro oxidation. Journal of the Science of Food and Agriculture 1990, 50, 547-561.

75. Kuhnert, N.; Dairpoosh, F.; Yassin, G.; Golon, A.; Jaiswal, R. What is under the hump? Mass spectrometry based analysis of complex mixtures in processed food-lessons from the characterisation of black tea thearubigins, coffee melanoidines and caramel. Food and Function 2013, 4, 1130-1147.

76. Pelillo, M.; Cuvelier, M. E.; Biguzzi, B.; Gallina Toschi, T.; Berset, C.; Lercker, G. Calculation of the molar absorptivity of polyphenols by using liquid chromatography with diode array detection: The case of carnosic acid. Journal of Chromatography A 2004, 1023, 225-229.

77. Sang, S.; Tian, S.; Meng, X.; Stark, R. E.; Rosen, R. T.; Yang, C. S.; Ho, C. T. Theadibenzotropolone A, a new type pigment from enzymatic oxidation of (-)-epicatechin and (-)-epigallocatechin gallate and characterized from black tea using LC/MS/MS. Tetrahedron Letters 2002, 43, 7129-7133.

78. Chen, H.; Shurlknight, K.; Leung, T.; Sang, S. Structural identification of theaflavin trigallate and tetragallate from black tea using liquid chromatography/electrospray ionization tandem mass spectrometry. Journal of Agricultural and Food Chemistry 2012, 60, 10850-10857.

79. Tanaka, T.; Kouno, I. Oxidation of tea catechins: Chemical structures and reaction mechanism. Food Science and Technology Research 2003, 9, 128-133.

80. Humphrey, A. M. Chlorophyll as a color and functional ingredient. Journal of Food Science 2004, 69, C422-C425.

81. Laddi, A.; Prakash, N. R.; Kumar, A. Quality evaluation of black CTC teas based upon seasonal variations International Journal of Food Science and Technology 2014, 49, 493-500.

82. Owuor, P. O.; Obanda, M.; Nyirenda, H. E.; Mphangwe, N. I. K.; Wright, L. P.; Apostolides, Z. The relationship between some chemical parameters and sensory evaluations for plain black tea (Camellia sinensis) produced in Kenya and comparison with similar teas from Malawi and South Africa. Food Chemistry 2006, 97, 644-653.

83. Hilton, P. J.; Ellis, R. T. Estimation of the market value of Central African tea by theaflavin analysis. Journal of the Science of Food and Agriculture 1972, 23, 227-232.

84. Narukawa, M.; Noga, C.; Ueno, Y.; Sato, T.; Misaka, T.; Watanabe, T. Evaluation of the bitterness of green tea catechins by a cell-based assay with the human bitter taste receptor hTAS2R39. Biochemical and Biophysical Research Communications 2011, 405, 620-625.

85. Chen, Q.; Guo, Z.; Zhao, J. Identification of green tea's (Camellia sinensis (L.)) quality level according to measurement of main catechins and caffeine contents by HPLC and support vector classification pattern recognition. Journal of Pharmaceutical and Biomedical Analysis 2008, 48, 1321-1325. 
86. Yamazaki, T.; Sagisaka, M.; Ikeda, R.; Nakamura, T.; Matsuda, N.; Ishii, T.; Nakayama, T.; Watanabe, T. The human bitter taste receptor hTAS2R39 is the primary receptor for the bitterness of theaflavins. Bioscience, Biotechnology and Biochemistry 2014, 78, 1753-1756.

87. Yamazaki, T.; Narukawa, M.; Mochizuki, M.; Misaka, T.; Watanabe, T. Activation of the HTAS2R14 human bitter-taste receptor by (-)-epigallocatechin gallate and (-)-epicatechin gallate. Bioscience, Biotechnology and Biochemistry 2013, 77, 1981-1983.

88. Jöbstl, E.; Fairclough, J. P. A.; Davies, A. P.; Williamson, M. P. Creaming in black tea. Journal of Agricultural and Food Chemistry 2005, 53, 7997-8002.

89. Brown, P. J.; Wright, W. B. An Investigation of the interactions between milk proteins and tea polyphenols. Journal of Chromatography A 1963, 11, 504-514.

90. Kaneko, S.; Kumazawa, K.; Masuda, H.; Henze, A.; Hofmann, T. Molecular and sensory studies on the umami taste of Japanese green tea. Journal of Agricultural and Food Chemistry 2006, 54, 2688-2694.

91. Gibbins, H. L.; Carpenter, G. H. Alternative mechanisms of astringency - What is the role of saliva? Journal of Texture Studies 2013, 44, 364-375.

92. Jöbstl, E.; O'Connell, J.; Fairclough, J. P. A.; Williamson, M. P. Molecular model for astringency produced by polyphenol/protein interactions. Biomacromolecules 2004, 5, 942-949.

93. Nayak, A.; Carpenter, G. H. A physiological model of tea-induced astringency. Physiology and Behavior 2008, 95, 290-294.

94. Canon, F.; Giuliani, A.; Paté, F.; Sarni-Manchado, P. Ability of a salivary intrinsically unstructured protein to bind different tannin targets revealed by mass spectrometry. Analytical and Bioanalytical Chemistry 2010, 398, 815-822.

95. Plet, B.; Delcambre, A.; Chaignepain, S.; Schmitter, J. M. Affinity ranking of peptide-polyphenol noncovalent assemblies by mass spectrometry approaches. Tetrahedron 2015, 71, 3007-3011.

96. Spencer, C. M.; Cai, Y.; Martin, R.; Gaffney, S. H.; Goulding, P. N.; Magnolato, D.; Lilley, T. H.; Haslam, E. Polyphenol complexation-some thoughts and observations. Phytochemistry 1988, 27, 2397-2409.

97. De Freitas, V.; Mateus, N. Nephelometric study of salivary protein-tannin aggregates. Journal of the Science of Food and Agriculture 2002, 82, 113-119.

98. Bhuyan, L. P.; Borah, P.; Sabhapondit, S.; Gogoi, R.; Bhattacharyya, P. Spatial variability of theaflavins and thearubigins fractions and their impact on black tea quality. Journal of Food Science and Technology 2015, 52, 7984-7993.

99. Owuor, P. O.; Obanda, M. Clonal variation in the individual theaflavin levels and their impact on astringency and sensory evaluations. Food Chemistry 1995, 54, 273-277.

100. Schwarz, B.; Hofmann, T. Is there a direct relationship between oral astringency and human salivary protein binding? European Food Research and Technology 2008, 227, 1693-1698.

101. Couzinet-Mossion, A.; Balayssac, S.; Gilard, V.; Malet-Martino, M.; Potin-Gautier, M.; Behra, P. Interaction mechanisms between caffeine and polyphenols in infusions of Camellia sinensis leaves. Food Chemistry 2010, 119, 173-181.

102. Owuor, P. O.; Reeves, S. G.; Wanyoko, J. K. Correlation of theaflavins content and valuations of Kenyan black teas. Journal of the Science of Food and Agriculture 1986, 37, 507-513.

103. El-Shahawi, M. S.; Hamza, A.; Bahaffi, S. O.; Al-Sibaai, A. A.; Abduljabbar, T. N. Analysis of some selected catechins and caffeine in green tea by high performance liquid chromatography. Food Chemistry 2012, 134, 2268-2275.

104. Vuong, Q. V.; Bowyer, M. C.; Roach, P. D. L-Theanine: Properties, synthesis and isolation from tea. Journal of the Science of Food and Agriculture 2011, 91, 1931-1939. 
Chapter 1 


\title{
Chapter 2
}

\section{Altering the phenolics profile of a green tea leaves extract using exogenous oxidases}

\begin{abstract}
Transformation from green tea leaves into black tea involves oxidation of catechins into theaflavins and other complex phenolics by endogenous enzymes in tea leaves. By employing tyrosinase and laccase, both from Agaricus bisporus, on green tea catechins, the oxidation process was directed towards a higher theaflavins content, which is considered an important quality parameter in tea. The main tea catechins were incubated with tyrosinase and laccase, and product formation was monitored by RP-UHPLC-PDA-ESI-MS. The kind of catechin, their substitution with a galloyl group, and the type of oxidase used were important factors determining theaflavin concentrations. In particular, incubation of epicatechin with epigallocatechin with tyrosinase gave a high, stable theaflavin content. In a green tea extract, tyrosinase increased the proportion of theaflavins by two-fold compared to black tea. Laccase mainly formed insoluble complexes. Our results indicate that the phenolic profile of tea can be modulated by using commercially available exogenous oxidases.
\end{abstract}

Based on: Annewieke J.W. Verloop, Harry Gruppen, Robbin Bisschop, Jean-Paul Vincken. Food Chemistry, 2016, 196, 1197-1206. 


\subsection{Introduction}

Processing of green tea leaves to black tea involves oxidation of phenolics. During this oxidation the main phenolic compounds present in green tea leaves, catechins (up to $30 \%$ DW), are oxidized into complex phenolics by the endogenous oxidative enzymes. Catechins belong to the group of flavan-3-ols, characterised by their meta-5,7-dihydroxy substituted A-ring and di- or trihydroxylic B-ring. The four major catechins in tea leaves are (-)-epigallocatechin gallate (EGCg), (-)-epicatechin gallate (ECg), (-)-epigallocatechin (EGC) and (-)-epicatechin (EC).

In black tea, only $10 \%$ of the green tea catechins are left. The oxidation products of the other $90 \%$ of catechins are only partly known. Extensive research is done on the so-called thearubigins, which account for $70 \%$ of the phenolics in black tea. The molecular diversity amongst thearubigins is bewildering, and theories explaining their formation are currently developed. ${ }^{2}$ Precursor oxidation products of thearubigins in black tea are theaflavins, theacitrins, (dehydro)theasinensins, theaflagallins, theaflavic acids and theanaphtoquinones, the structure of which has been clearly defined. ${ }^{3}$ Taken together, these groups account for $20 \%$ of the total phenolic content of black tea, of which the theaflavins are most abundant, comprising one third of these phenolics. ${ }^{1,3}$ Consequently, theaflavins are the main phenolic compounds investigated in black tea. Theaflavins have been reported to contribute to the specific colour ${ }^{4-6}$ and astringency ${ }^{7}$ of black tea. Their content correlates well with the quality and selling price of black tea. ${ }^{8}$ Theaflavins are oxidative condensation products of a catechin and a gallocatechin. ${ }^{9}$ Depending on the catechins used, with or without galloyl group, theaflavin (TF), theaflavin gallate (TFg) or theaflavin digallate (TFgg) are formed. Theaflavins can participate in subsequent reactions, either by enzymatic oxidation or by non-enzymatic coupled oxidation involving catechin quinones. In the latter case electrons are transferred from the theaflavin to the catechin quinone, which then regenerates into catechin. ${ }^{10}$ Considering the theaflavin's contribution to black tea quality, the subsequent reactions are undesirable. Although the formation of theaflavins from catechins and gallocatechins, and the routes towards further reaction products have been described, ${ }^{3}$ it is still unclear which oxidative enzymes are responsible for the theaflavin formation.

In tea leaves, polyphenol oxidase (PPO) and peroxidase activities are known to occur. ${ }^{11}$ PPOs generally include (i) oxidative enzymes with cresolase activity, like tyrosinase, that catalyse the $o$-hydroxylation of monophenols into $o$-diphenols and subsequent oxidation of $o$-diphenols into $o$-quinones, and (ii) enzymes with catecholase activity that only catalyse the oxidation of $o$-diphenols into $o$-quinones. Laccases are also considered PPOs by some. $^{12,13}$ Often the term PPO is not defined, albeit differences between laccases and oxidative enzymes with cresolase or catecholase activity exist. Laccases form a radical upon oxidation and only after two such reactions a quinone is formed. Laccases also have 
much wider substrate specificity than only $o$-diphenols. For instance, $p$-diphenols, triphenols and even lignin are also oxidized. ${ }^{14}$

With few exceptions, ${ }^{12,15}$ the type of oxidase activity is not specified in studies on tea leaf PPOs. This specification of activity is important considering the differences between cresolase, catecholase and laccase. For instance, cresolase is expected to act only on the $o$ dihydroxylic B-ring of EC and ECg. Laccases can potentially also act on the trihydroxylic B-ring of EGC and EGCg and on the galloyl group attached to the C-ring of ECg and EGCg. Due to this wider substrate specificity laccase is expected to oxidize theaflavins as well. Therefore, laccase activity will result in lower theaflavin content and, from this perspective, does not seem to be beneficial to black tea quality.

The aim of this study is to direct the oxidation of tea catechins towards increased theaflavin content, starting from a green tea extract. Increasing theaflavin content starting from the green tea leaves, by adding tyrosinase, has already been described in a patent. ${ }^{16}$ Moreover, in several studies tea catechin solutions have been treated with an enzyme preparation endogenous to tea leaves, referred to as PPO, but it was undefined whether it contained catecholase, cresolase or laccase activities, or combinations thereof ${ }^{8,17,18}$ To characterize the potential contribution of catecholase, cresolase and laccase to altering the phenolics profile of green tea, distinct activities should be used. As oxidative enzymes from tea leaves are not available individually, specific oxidative enzymes from exogenous origin were used in this research. Tyrosinase (cresolase activity; pure catecholase activity could not be purchased) and laccase, both from Agaricus bisporus, were incubated with green tea catechins, and their effects on theaflavin formation and stability were compared.

\subsection{Materials and Methods}

\subsubsection{Materials}

$(+)$-Catechin (C), (-)-epicatechin (EC), (-)-epigallocatechin gallate (EGCg), theaflavin3 -gallate (TFg), L-ascorbic acid and mushroom tyrosinase were purchased from Sigma Aldrich (St. Louis, MO, USA). Laccase from Agaricus bisporus was purchased from Fluka (Sigma Aldrich), anhydrous citric acid from Acros Organics (Geel, Belgium) and dipotassium hydrogen phosphate from Merck (Darmstadt, Germany). (-)-Epigallocatechin (EGC) was prepared from EGCg according to ${ }^{19} .2,5$-Dihydroxybenzoic acid (DHB) was purchased from Bruker Daltronics (Bremen, Germany). AG 50W-X8 Resin was purchased from BioRad Laboratories (Hercules, CA, USA). Maltodextrin MD20 (DP 1 to 20) was obtained from Avebe (Veendam, The Netherlands). Ultra-high-performance liquid chromatography mass spectrometry (UHPLC-MS) grade acetonitrile (ACN) was obtained from Biosolve BV (Valkenswaard, The Netherlands). Water was prepared using a Milli-Q 
water purification system (Millipore, Billerica, MA, USA). Fresh tea leaves (Camellia sinensis var. assamica) from Kenya were kindly provided by Unilever R\&D (Vlaardingen, The Netherlands).

\subsubsection{Extraction of tea phenolics}

Green tea: Fresh tea leaves $(2 \mathrm{~g})$ were cut and crushed with mortar and pestle and subsequently three times extracted with $50 \mathrm{~mL}$ boiling water. Extracts were filtered through paper filter (Grade 1, Whatman, Chalfont St. Giles, UK) by Büchner filtration and the filtrates were combined.

Black tea: Fresh tea leaves $(2 \mathrm{~g})$ were cut, crushed with mortar and pestle and left in open air to oxidize for $24 \mathrm{~h}$. The extraction procedure was the same as described for green tea.

\subsubsection{Incubation of tyrosinase or laccase with catechins or green tea extract}

EC, C, EGC, EGCg, and combinations thereof were used as substrates at a final equimolar total substrate concentration of $0.1 \mathrm{mM}$. Either tyrosinase (cresolase activity; $0.0755 \mathrm{U} / \mathrm{mg})$ or laccase $(0.0015 \mathrm{U} / \mathrm{mg})$ was added to a final concentration of $0.0001 \mathrm{U} / \mathrm{mL}$ (one unit equalling the amount of enzyme that consumes $1 \mu \mathrm{mol}$ of oxygen per min for oxidation of catechin at $\mathrm{pH} 5.5$ and $25{ }^{\circ} \mathrm{C}$ ). Incubations were performed in citric acidpotassium phosphate buffer $(30 \mathrm{mM}) \mathrm{pH} 5.5$. Samples $(1 \mathrm{~mL})$ were incubated at $25^{\circ} \mathrm{C}$ for $5,10,15,30,60,90$ and $120 \mathrm{~min}$, after which the reaction was stopped by adding $20 \mu \mathrm{L}$ of freshly prepared saturated ascorbic acid solution. The amount of ascorbic acid added was sufficient to arrest the reaction for at least $12 \mathrm{~h}$. Green tea extract was mixed with either tyrosinase or laccase $(0.005 \mathrm{U} / \mathrm{mL})$. Samples $(1 \mathrm{~mL})$ were incubated at $25{ }^{\circ} \mathrm{C}$ for $24 \mathrm{~h}$. Incubations longer than $24 \mathrm{~h}$, with more enzyme or with a second enzyme addition, did not result in different phenolic profiles compared to the $24 \mathrm{~h}$ sample. After incubation the reactions were stopped as described above. The samples were centrifuged $(10.000 \mathrm{~g}, 5 \mathrm{~min}$, $20^{\circ} \mathrm{C}$ ) prior to analysis by reversed-phase liquid chromatography. For spectrophotometric analysis, the same samples were analysed in a UV-1601 spectrophotometer (Shimadzu, Kyoto, Japan). Wavelength scans were made from $380-780 \mathrm{~nm}$.

Compounds were identified by mass spectrometry and quantified by spectrophotometric responses. A calibration curve of EC at $\lambda 270 \mathrm{~nm}$ was used to calculate the concentrations of the compounds by using their molecular extinction coefficients: EC 4,290 L/(mol.cm); C 2,484 L/(mol $\cdot \mathrm{cm})$; EGC 2,088 L/(mol.cm); EGCg 11,920 L/(mol $\cdot \mathrm{cm})^{20}$ and TF 21,400 $\mathrm{L} /(\mathrm{mol} \cdot \mathrm{cm})$; TFg 30,500 L/(mol $\cdot \mathrm{cm})$; TFgg 35,200 L/(mol $\cdot \mathrm{cm}) .{ }^{21}$ For compounds of which the molar extinction coefficient is unknown, the molar extinction coefficient of the 
compound with the closest resemblance was used. For theanaphtoquinone (TNQ), dehydrotheasinensin C (DHTS C) and T3D (TF-EC) the molar extinction coefficient of TF was used. For TNQg and T3Dg (TFg-EC) the molar extinction coefficient of TFg was used.

\subsubsection{RP-UHPLC}

Samples were analysed on an Accela UHPLC system (Thermo Scientific, San Jose, CA, USA) equipped with a pump, an auto sampler at $15{ }^{\circ} \mathrm{C}$ and a photodiode array (PDA) detector. Samples $(1 \mu \mathrm{L})$ were injected onto a Hypersil Gold column $(2.1 \times 150 \mathrm{~mm}$, particle size of $1.9 \mu \mathrm{m}$, Thermo Scientific). Water containing $1 \%(\mathrm{v} / \mathrm{v}) \mathrm{ACN}$ and $0.1 \%(\mathrm{v} / \mathrm{v})$ acetic acid (eluent A) and ACN containing $0.1 \%(\mathrm{v} / \mathrm{v})$ acetic acid (eluent B) were used as eluents. The flow rate was $400 \mu \mathrm{L} / \mathrm{min}$, and the column oven temperature was controlled at $30{ }^{\circ} \mathrm{C}$. The PDA detector was set to measure $200-600 \mathrm{~nm}$. The following elution profile was used: $0-1 \mathrm{~min}$, isocratic on $5 \%(\mathrm{v} / \mathrm{v}) \mathrm{B} ; 1-20 \mathrm{~min}$, linear gradient from $5-100 \%(\mathrm{v} / \mathrm{v}) \mathrm{B}$; 20-25 min, isocratic on $100 \%(\mathrm{v} / \mathrm{v}) \mathrm{B} ; 25-26 \mathrm{~min}$, linear gradient from $100-5 \%(\mathrm{v} / \mathrm{v}) \mathrm{B}$; and 26-31 min, isocratic on $5 \%(\mathrm{v} / \mathrm{v}) \mathrm{B}$.

\subsubsection{Electrospray Ionization - Mass Spectrometry (ESI-MS)}

Mass spectrometric data were obtained by analysing samples on a LTQ Velos mass spectrometer (Thermo Scientific) equipped with an ESI probe coupled to the RP-UHPLC system. Nitrogen was used as sheath gas and auxiliary gas. Data were collected over the $\mathrm{m} / \mathrm{z}$ range of 250-2000. Data-dependent $\mathrm{MS}^{\mathrm{n}}$ analysis was performed with a normalized collision energy of $35 \%$. The $\mathrm{MS}^{\mathrm{n}}$ fragmentation was performed on the most intense product ion in the $\mathrm{MS}^{\mathrm{n}-1}$ spectrum, with a dynamic exclusion for $5 \mathrm{~s}$, when the most intense product ion appeared twice within $5 \mathrm{~s}$. The system was tuned with theaflavin-3'-gallate in negative ionization mode. Most settings were optimized via automatic tuning using 'Tune Plus' (Xcalibur 2.07, Thermo Scientific). The transfer tube temperature was $350{ }^{\circ} \mathrm{C}$, and the source voltage was $4.0 \mathrm{kV}$. Data acquisition and reprocessing were performed with Xcalibur 2.07 (Thermo Scientific).

\subsubsection{Oxygen consumption measurements}

Oxygen consumption by the enzymes upon incubation with substrates was measured using an Oxytherm system (Hansatech, Kings Lynn, U.K.). Incubations with single catechins $\mathrm{C}, \mathrm{EC}, \mathrm{EGC}, \mathrm{EGCg}$ or TFg $(1 \mathrm{mM})$ and $0.001 \mathrm{U}$ of tyrosinase or laccase were performed at $25{ }^{\circ} \mathrm{C}$ in a total volume of $1 \mathrm{~mL} 30 \mathrm{mM}$ citric acid - potassium phosphate buffer $\mathrm{pH}$ 5.5. Control incubations containing substrates without enzymes were performed 
as well, and their respective oxygen consumption values were subtracted from the measured enzyme activities. Data acquisition and analysis were performed using $\mathrm{O}_{2}$ view software (Hansatech).

\subsubsection{MALDI-TOF-MS}

Samples were prepared as described and subsequently desalted prior to analysis using AG $50 \mathrm{~W}-\mathrm{X} 8$ resin. A solution of $10 \mathrm{mg} / \mathrm{mL}$ of DHB in water was used as matrix. Spots were prepared on a MTP 384 ground steel target plate (Bruker Daltonics): $0.5 \mu \mathrm{L}$ of DHB solution was applied and dried under a stream of air, then $0.5 \mu \mathrm{L}$ of sample was applied on top of it and dried under a stream of air, finally $0.5 \mu \mathrm{L}$ of DHB solution was applied on top of it and dried under a stream of air. Samples were analysed on an Ultraflextreme workstation equipped with a Smartbeam II laser of $355 \mathrm{~nm}$, operated in positive mode and controlled by FlexControl 3.4 software (Bruker Daltonics). Spectra were collected at a laser intensity between $55 \%$ and $65 \%$ in reflector mode with voltages of ion source 1 and 2, reflector 1 and 2 and the lens; $25.01 \mathrm{kV}, 22.37 \mathrm{kV}, 26.45 \mathrm{kV}, 13.32 \mathrm{kV}$ and $8.34 \mathrm{kV}$ respectively. Laser frequency was $1000 \mathrm{~Hz}$. Calibration was performed with maltodextrin and a mass scan range from $200-3500 \mathrm{Da}$ was used with matrix suppression up to $250 \mathrm{Da}$. Data analysis was conducted using FlexAnalysis v3.3 software (Bruker Daltonics).

\subsection{Results and Discussion}

\subsubsection{Tyrosinase and laccase activity compared on individual tea catechins}

To compare the activity of tyrosinase (cresolase activity) and laccase on individual catechins, the amount of enzyme added to the incubation was standardized to a similar oxygen consumption rate, $0.001 \mu \mathrm{mol} / \mathrm{mL}$ per min, on catechin. Figure 2.1 shows the rate of oxygen consumption of tyrosinase and laccase on the different catechins. Both enzymes showed $\sim 1.5$ times higher activity towards $\mathrm{EC}$ than towards $\mathrm{C}$, in accordance to literature on tea catecholase. ${ }^{12}$ For tyrosinase, activities on EGC and EGCg were 14 and 7 times lower, respectively, than that on C. For laccase, activity on EGC was 4 times lower, whereas activity on EGCg was roughly similar to that on $\mathrm{C}$. The much lower activities of tyrosinase on EGC and EGCg compared to activity on C were expected, because the former phenolics have been found to inhibit tyrosinase from Agaricus bisporus. ${ }^{22}$ When comparing the enzymes, laccase showed $\sim 3$ and $\sim 6$ times higher activities towards EGC and EGCg, respectively, than tyrosinase. The activity of laccase on EGC and EGCg was expected, because of its wider substrate specificity. The activity of tyrosinase towards trihydroxylic 
aromatic rings, although low in comparison to laccase and to activity on $\mathrm{EC}$, has not been described before to our knowledge.

Previously, for tea PPO extracts, a 1.5 times higher activity on EGC compared to that on $\mathrm{C}$ was measured by oxygen consumption measurements, and a roughly similar activity on EGCg compared to that on $\mathrm{C}$, or a 3 times higher activity on EGCg compared to that on EC. ${ }^{12}$ Our results suggest that those PPO extracts should also contain laccase activity, besides cresolase, otherwise the relatively high activity towards trihydroxylic substrates is difficult to explain.

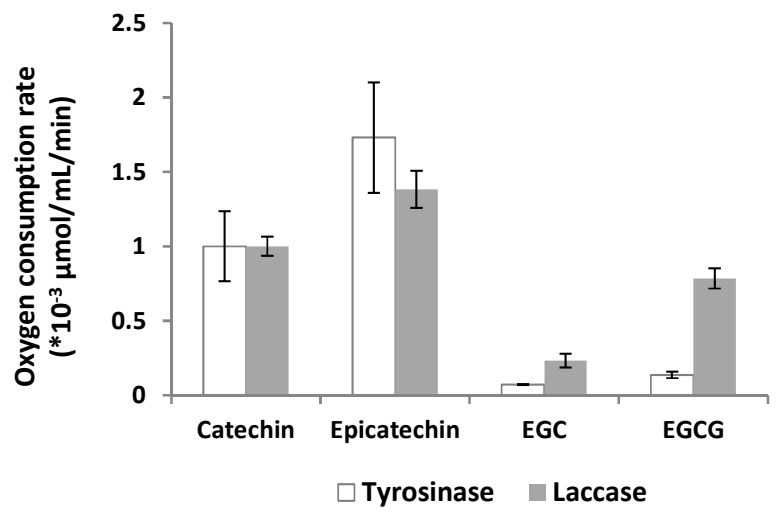

Figure 2.1 Oxygen consumption rate $(\mu \mathrm{mol} / \mathrm{mL} / \mathrm{min})$, normalized to $\mathrm{C}$, of tyrosinase and laccase incubated with individual catechins.

\subsubsection{Conversion of individual tea catechins by tyrosinase and laccase compared}

After one hour of incubation with either tyrosinase or laccase, $85 \%$ of both $\mathrm{C}$ and $\mathrm{EC}$ had reacted. For tyrosinase, only $10 \%$ of EGC and $37 \%$ of EGCg had reacted (data not shown). This lower conversion might be explained by the inhibitory effect of these compounds mentioned above. For laccase, 63\% of EGC reacted, whereas EGCg was completely consumed. These results are not fully in line with the oxygen consumption measurements, where a higher activity on $\mathrm{C}$ and $\mathrm{EC}$ compared to $\mathrm{EGCg}$ was found for laccase (Fig. 2.1). This apparent discrepancy might be explained by the fact that oxygen consumption measurements represent the initial reaction rate on the substrates, whereas the LC-MS analysis represents the composition of phenolics after one hour of incubation. Alternatively, the oxidation products of $\mathrm{C}$ and EC's individual incubations might have an inhibiting effect on laccase. 


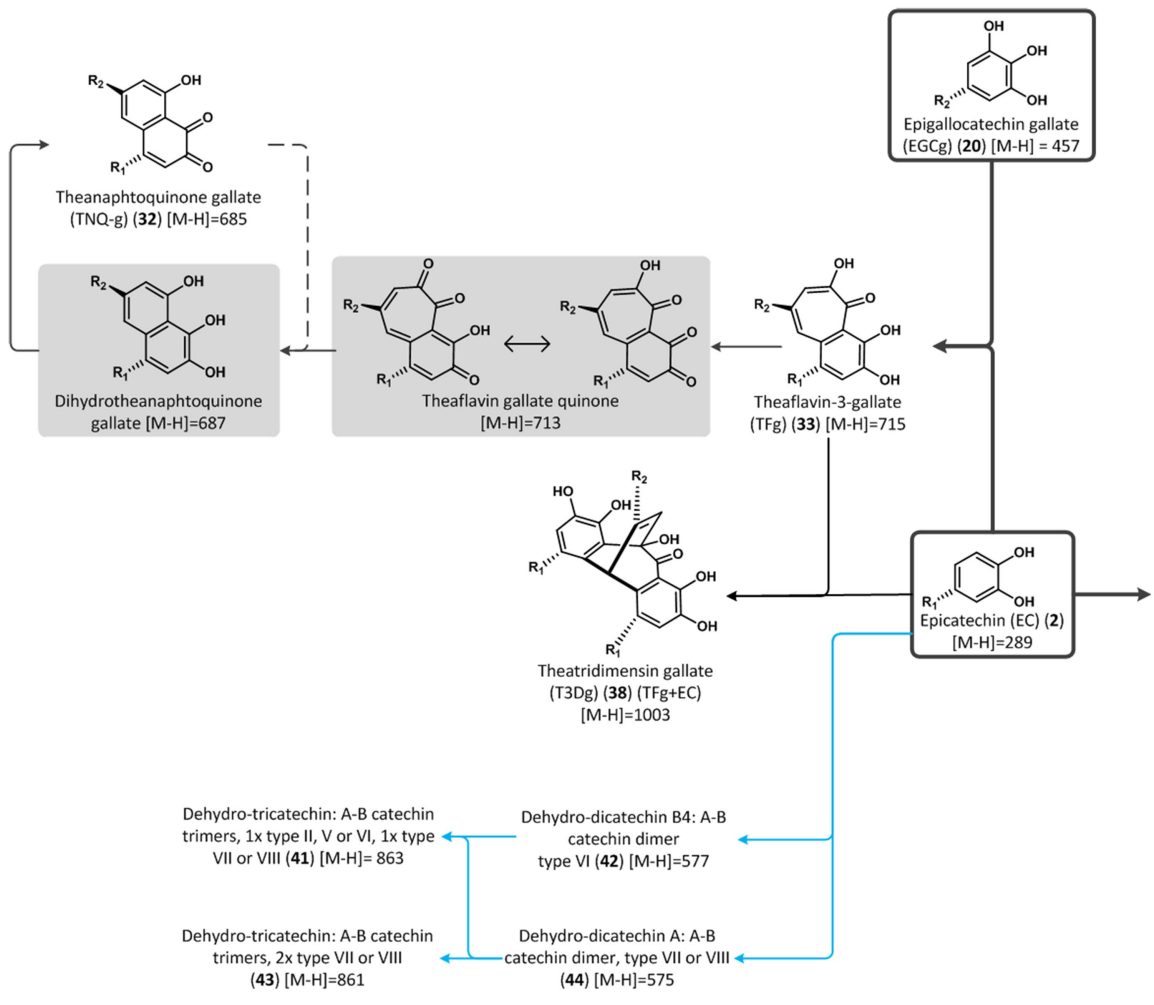

Figure 2.2 Schematic representation of the reaction products found in the model incubations of tyrosinase and laccase with individual catechins (blue pathways) and combinations of catechins (black pathways). Pathways were drawn according to literature. ${ }^{3,5,23}$ Boxed compounds are the initial catechins used in this study, compounds in grey were intermediates not found in the present study, dotted lines are hypothetical reaction pathways. For C and EC the same compounds (stereo isomers) were formed, so only EC is included in the figure. The compounds are numbered according to Table 2.1. 
Altering the phenolics profile of tea with tyrosinase or laccase

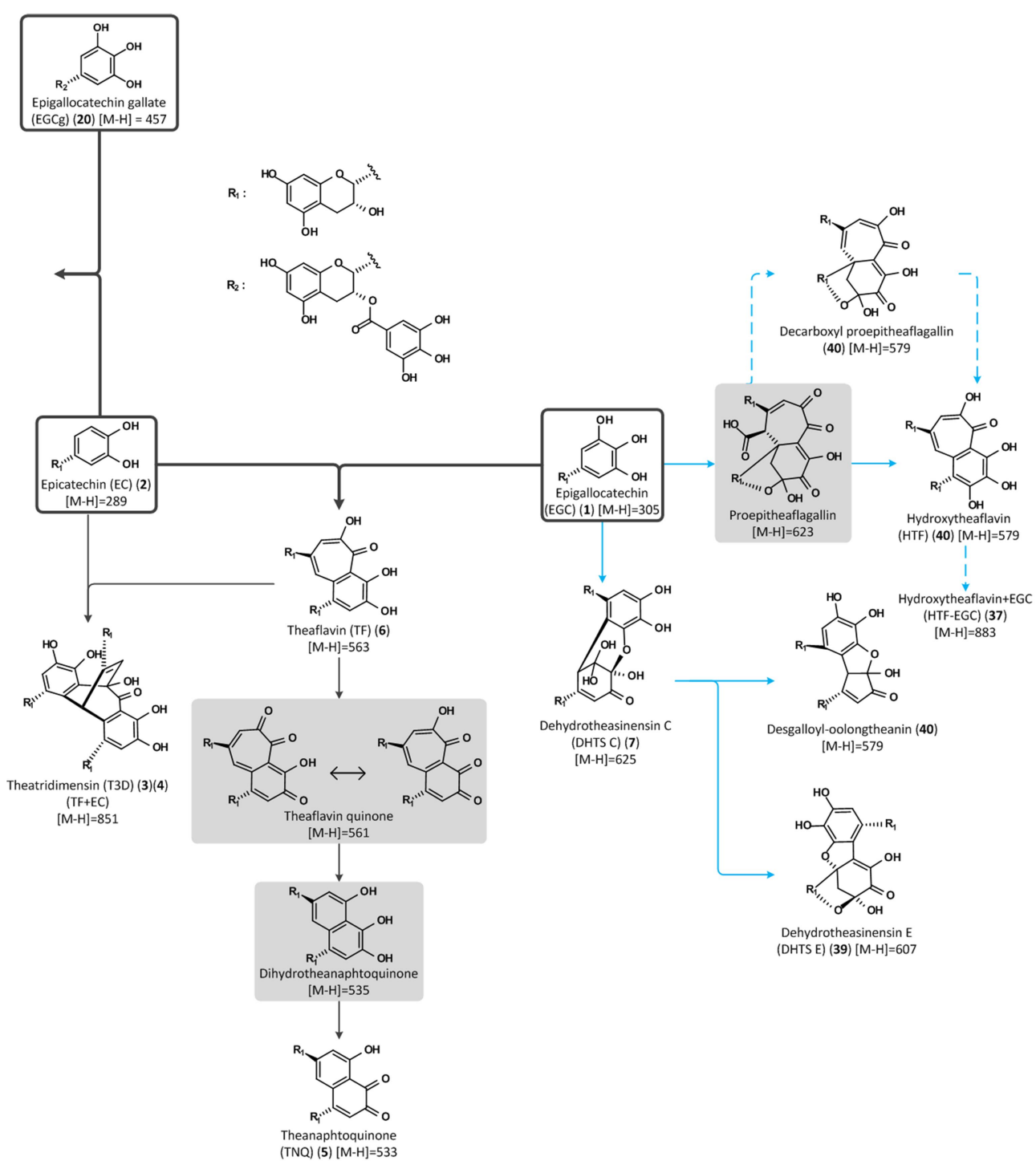

Continuation of Figure 2.2. 


\subsubsection{Characterization of enzymatic oxidation products of individual tea catechins}

The peaks in the RP-UHPLC chromatograms were annotated based on comparison of MS/MS fragmentation with published data (Table 2.1). In Figure 2.2 a schematic overview is given, summarizing in blue the compounds formed in the single catechin incubations. The pathways to the different oxidation products were drawn according to literature. ${ }^{3,5,23}$

Incubations of $\mathrm{C}$ or $\mathrm{EC}$ with tyrosinase or laccase resulted in dimers and trimers, coupled by the A-ring to the B-ring, the dehydrodicatechins and dehydrotricatechins. ${ }^{24}$ Incubations of EGC with tyrosinase or laccase resulted in dimers as well, i.e. the dehydrotheasinensins and a product with an $\mathrm{m} / \mathrm{z}$ value of 579 (40). The latter can either be hydroxytheaflavin, decarboxyl proepitheaflagallin, or desgalloyl oolongtheanin (Fig. 2.2). The mass fragmentation patterns of these isomers have not been described yet. Therefore, the incubation mixture was checked for the presence of the known precursors of the three isomers. Desgalloyl oolongtheanin is formed from dehydrotheasinensin C (DHTS C) (7), which has an $m / z$ value of $625 .{ }^{23}$ DHTS C was present in the incubation (Table 2.1). The other two isomers, hydroxytheaflavin and decarboxyl proepitheaflagallin, are both formed from the same precursor, proepitheaflagallin, which has an $m / z$ value of $623 .{ }^{23}$ This compound was not present in the incubation. Therefore, the $\mathrm{m} / \mathrm{z} 579$ compound most likely corresponds to desgalloyl oolongtheanin. To confirm this, the MS/MS fragments of the $\mathrm{m} / z$ 579 compound were compared with those of the precursor. The mass difference between DHTS C and desgalloyl oolongtheanin is $46 \mathrm{Da}$. If the compound with $\mathrm{m} / \mathrm{z}$ value 579 is desgalloyl oolongtheanin, mass fragments with a 46 Da difference to that of DHTS C are expected in the MS/MS spectrum. DHTS C showed a fragment of $m / z 565 \mathrm{Da}$, which can be correlated to a fragment of $\mathrm{m} / z 519$ of the putative desgalloyl oolongtheanin, by the 46 Da difference. Therefore, the compound with $m / z$ value 579 was tentatively assigned to be desgalloyl oolongtheanin.

Table 2.1. Compounds tentatively assigned by RP-UHPLC-MSn from the model incubations of the catechins with tyrosinase or laccase, and the green tea incubations with tyrosinase or laccase.

\begin{tabular}{|c|c|c|c|c|c|c|}
\hline $\begin{array}{c}\text { Peak } \\
\text { no. }\end{array}$ & $\begin{array}{l}\text { Retention } \\
\text { time (min) }\end{array}$ & $\begin{array}{c}{[\mathrm{M}-\mathrm{H}]^{-}} \\
m / z\end{array}$ & $\begin{array}{c}\text { MS }^{2} \text { fragments } \\
\qquad \mathrm{m} / z\end{array}$ & $\begin{array}{c}\lambda_{\max } \\
(\mathrm{nm})^{*}\end{array}$ & Tentative identification $^{\dagger}$ & $\begin{array}{c}\text { Model } \\
\text { Incubation }\end{array}$ \\
\hline \multicolumn{7}{|c|}{ Catechins } \\
\hline $1^{\neq}$ & 4.37 & 305 & $\begin{array}{l}179,219,221, \\
261\end{array}$ & 268 & Epigallocatechin $^{29}$ & \\
\hline $2^{\neq}$ & 5.53 & 289 & 245,205 & 278 & Epicatechin $^{29}$ & \\
\hline 9 & 2.73 & 305 & $\begin{array}{l}179,219,221, \\
261\end{array}$ & 270 & Gallocatechin $^{29}$ & \\
\hline 17 & 4.84 & 289 & 245,205 & 280 & Catechin $^{29}$ & \\
\hline
\end{tabular}


Altering the phenolics profile of tea with tyrosinase or laccase

\begin{tabular}{|c|c|c|c|c|c|c|}
\hline $\begin{array}{c}\text { Peak } \\
\text { no. }\end{array}$ & $\begin{array}{r}\text { Retention } \\
\text { time (min) }\end{array}$ & $\begin{array}{c}{[\mathrm{M}-\mathrm{H}]^{-}} \\
\mathrm{m} / z\end{array}$ & $\begin{array}{c}\mathrm{MS}^{2} \text { fragments } \\
\mathrm{m} / \mathrm{z}\end{array}$ & $\begin{array}{r}\lambda_{\max } \\
(\mathbf{n m})^{*}\end{array}$ & Tentative identification $^{\dagger}$ & $\begin{array}{c}\text { Model } \\
\text { Incubation }\end{array}$ \\
\hline 20 & 5.73 & 457 & $169,331,305$ & 274 & Epigallocatechin gallate $^{29}$ & \\
\hline 26 & 6.70 & 441 & 289,169 & 274 & $\left(\right.$ Epi-)catechin gallate ${ }^{29}$ & \\
\hline 29 & 7.41 & 425 & $273,169,255$ & 268,358 & Epiafzelechin gallate $^{30}$ & \\
\hline \multicolumn{7}{|c|}{ Theaflavins and theaflavin derivatives } \\
\hline $3^{*}$ & 5.59 & 851 & $\begin{array}{l}617,713,815 \\
707,695\end{array}$ & n.d. & $\begin{array}{l}\text { Theatridimensin }{ }^{3 l} \\
\text { (TF-EC) }\end{array}$ & $\begin{array}{l}\text { EC+EGC } \\
\text { (Tyr) }\end{array}$ \\
\hline $4^{*}$ & 5.90 & 851 & $\begin{array}{l}617,713,815 \\
707,695\end{array}$ & n.d. & $\begin{array}{l}\text { Theatridimensin }{ }^{31} \\
\text { (TF-EC) }\end{array}$ & $\begin{array}{l}\text { EC+EGC } \\
\text { (Tyr) }\end{array}$ \\
\hline $6^{*}$ & 8.08 & 563 & $\begin{array}{l}379,407,425, \\
241\end{array}$ & $\begin{array}{c}266, \\
375,452\end{array}$ & Theaflavin $^{32}$ & $\mathrm{EC}+\mathrm{EGC}$ \\
\hline 30 & 7.56 & 563 & $\begin{array}{l}407,379,481 \\
425,241\end{array}$ & 267,375 & Neotheaflavin $^{33}$ & $\mathrm{C}+\mathrm{EGC}$ \\
\hline 33 & 8.47 & 715 & $563,527,545$ & 271,375 & Theaflavin-3-gallate ${ }^{32}$ & $\mathrm{EC}+\mathrm{EGCg}$ \\
\hline 34 & 8.73 & 715 & $\begin{array}{l}563,407,545 \\
527\end{array}$ & 273,375 & Theaflavin-3'-gallate ${ }^{32}$ & \\
\hline 35 & 10.07 & 867 & $697,715,527$ & 273,376 & Theaflavin-digallate $^{2}$ & \\
\hline 36 & 8.29 & 715 & $563,545,527$ & n.d. & Neotheaflavin-3-gallate ${ }^{34}$ & $\begin{array}{l}\mathrm{C}+\mathrm{EGCg} \\
(\mathrm{Tyr})\end{array}$ \\
\hline 37 & 4.85 & 883 & $\begin{array}{l}847,821,811 \\
839,803\end{array}$ & 264 & Hydroxytheaflavin-EGC? ${ }^{23}$ & $\mathrm{C}+\mathrm{EGC}$ \\
\hline 38 & 6.29 & 1003 & $\begin{array}{l}833,617,695 \\
865\end{array}$ & 272 & $\begin{array}{l}\text { Theatridimensin gallate }{ }^{31} \\
\text { (TFg-EC) }\end{array}$ & $\begin{array}{l}\text { EC+EGCg } \\
(\text { Tyr) }\end{array}$ \\
\hline \multicolumn{7}{|c|}{ Complex phenolics in fermented teas } \\
\hline $5^{*}$ & 7.86 & 533 & $\begin{array}{l}505,471,349 \\
305\end{array}$ & 260 & Theanaphtoquinone $^{35}$ & $\mathrm{EC}+\mathrm{EGC}$ \\
\hline 7 & 1.63 & 625 & $\begin{array}{l}581,563,443 \\
565\end{array}$ & 268 & Dehydrotheasinensin $\mathrm{C}^{36}$ & $\begin{array}{l}\text { EGC, } \\
\text { EC+EGC, } \\
\text { C+EGC }\end{array}$ \\
\hline 8 & 2.03 & 609 & $\begin{array}{l}423,441,305 \\
483\end{array}$ & n.d. & Theasinensin ${ }^{2}$ & \\
\hline 10 & 3.28 & 759 & 607 & 285 & Theacitrin gallate $^{2}$ & \\
\hline 12 & 3.36 & 609 & $\begin{array}{l}423,441,305 \\
483\end{array}$ & 273 & Theasinensin ${ }^{2}$ & \\
\hline 13 & 4.02 & 759 & $607,427,589$ & 268 & Theacitrin gallate $^{2}$ & \\
\hline 15 & 4.68 & 759 & $\begin{array}{l}607,589,723 \\
427\end{array}$ & 276 & Theacitrin gallate $^{2}$ & \\
\hline 18 & 5.15 & 577 & $\begin{array}{l}425,407,451, \\
289\end{array}$ & 278 & $\begin{array}{l}\text { B-type proanthocyanidin } \\
\text { dimer }^{37,38}\end{array}$ & \\
\hline 32 & 8.28 & 685 & $\begin{array}{l}559,519,603 \\
507\end{array}$ & 267 & Theanaphtoquinone-gallate $^{5}$ & $\mathrm{EC}+\mathrm{EGCg}$ \\
\hline 39 & 5.36 & 607 & $\begin{array}{l}563,525,546, \\
439\end{array}$ & n.d. & Dehydrotheasinensin $\mathrm{E}^{10}$ & EGC \\
\hline
\end{tabular}




\begin{tabular}{|c|c|c|c|c|c|c|}
\hline $\begin{array}{c}\text { Peak } \\
\text { no. }\end{array}$ & $\begin{array}{l}\text { Retention } \\
\text { time (min) } \\
\end{array}$ & $\begin{array}{c}{[\mathrm{M}-\mathrm{H}]^{-}} \\
\mathrm{m} / \mathrm{z}\end{array}$ & $\begin{array}{c}\mathrm{MS}^{2} \text { fragments } \\
\mathrm{m} / z\end{array}$ & $\begin{array}{c}\lambda_{\max } \\
(\mathrm{nm})^{*}\end{array}$ & Tentative identification $^{\dagger}$ & $\begin{array}{c}\text { Model } \\
\text { Incubation } \\
\end{array}$ \\
\hline 40 & 5.65 & 579 & $\begin{array}{l}497,551,519, \\
413\end{array}$ & n.d. & $\begin{array}{l}\text { Hydroxytheaflavin/Desgallo } \\
\text { yl-oolongtheanin/Decarboxyl } \\
\text { proepitheaflagellin }\end{array}$ & EGC \\
\hline 41 & $\begin{array}{c}2.07,4.28, \\
4.69,4.94, \\
5.49,5.77 \\
5.95,6.54 \\
\quad 6.64\end{array}$ & 863 & $\begin{array}{l}725,711,682 \\
559,781\end{array}$ & n.d. & $\begin{array}{l}\text { Dehydro-tricatechin: A-B } \\
\text { ring catechin trimers, 1x type } \\
\text { II, V or VI, 1x type VII or } \\
\text { VIII }^{24}\end{array}$ & $\mathrm{C}$ \\
\hline 42 & $\begin{array}{l}4.47,4.74 \\
5.20,6.41\end{array}$ & 577 & $\begin{array}{l}439,393,425 \\
495,533,269 \\
517\end{array}$ & n.d. & $\begin{array}{l}\text { Dehydro-dicatechin B1-4: A- } \\
\text { B ring catechin dimer type } \\
\mathrm{VI}^{24}\end{array}$ & $\mathrm{C}, \mathrm{EC}$ \\
\hline 43 & $\begin{array}{c}4.96,5.47 \\
7.13\end{array}$ & 861 & $\begin{array}{l}799,817,697 \\
779,575,325 \\
283\end{array}$ & n.d. & $\begin{array}{l}\text { Dehydro-tricatechin: A-B } \\
\text { ring catechin trimer, } 2 x \text { type } \\
\text { VII or VIII }{ }^{24}\end{array}$ & \\
\hline 44 & $\begin{array}{c}6.35,7.10 \\
7.27\end{array}$ & 575 & $\begin{array}{l}493,449,515 \\
437,394,287\end{array}$ & n.d. & $\begin{array}{l}\text { Dehydro-dicatechin A: A-B } \\
\text { ring catechin dimer type VII } \\
\text { or } \mathrm{VIII}^{24}\end{array}$ & $\mathrm{C}$ \\
\hline \multicolumn{7}{|c|}{ Glycosylated flavonols } \\
\hline 21 & 6.04 & 479 & 316 & 266,353 & Myr-glc / myr-gal ${ }^{30}$ & \\
\hline 22 & 6.26 & 755 & 609 & 267,341 & $\begin{array}{l}\text { Kam-glc-rut / Que-di-rha- } \\
\text { glc }^{30}\end{array}$ & \\
\hline 23 & 6.37 & 609 & 301 & $\begin{array}{c}256 \\
264,353\end{array}$ & Que-rut $^{29}$ & \\
\hline 24 & 6.45 & 739 & 593 & 267,341 & Kam-di-cou-hex ${ }^{30}$ & \\
\hline 25 & 6.61 & 463 & 301 & $\begin{array}{c}256, \\
295,353\end{array}$ & Que-gal ${ }^{29}$ & \\
\hline 27 & 6.81 & 593 & 285 & 267,334 & Kam-cou-glc / kam-rha-gal ${ }^{30}$ & \\
\hline 28 & 7.07 & 447 & 284,285 & 266,346 & $\begin{array}{l}\text { Kam-gal / kam-glc / kam- } \\
\text { rut }^{29}\end{array}$ & \\
\hline 31 & 7.88 & 901 & 755 & 268,313 & $\begin{array}{l}\text { Que-cou-glc-rut / kam-cou- } \\
\text { glc-rha-gal / kam-cou-di-rha- } \\
\text { hex }^{30}\end{array}$ & \\
\hline \multicolumn{7}{|c|}{ Phenolic acid derivatives } \\
\hline 11 & 3.31 & 353 & 191,179 & 323 & Caffeoylquinic acid ${ }^{30}$ & \\
\hline 14 & 4.53 & 401 & 179,341 & 285,300 & $\begin{array}{l}\text { Caffeoyl-galloylquinic } \\
\text { acid? }^{30}\end{array}$ & \\
\hline 16 & 4.78 & 353 & $173,179,191$ & 326 & Caffeoylquinic acid ${ }^{30}$ & \\
\hline 19 & 5.47 & 337 & 173,191 & 308 & $p$-coumaroylquinic acid ${ }^{30}$ & \\
\hline
\end{tabular}

* Only $\lambda_{\max }$ above $250 \mathrm{~nm}$ are shown, $\dagger$ kam: kaempferol, myr: myricetin, que: quercetin, gal: galactose, glc: glucose, hex: hexose, rha: rhamnose, rut: rutin, cou: coumaroyl, ‡ peaks were assigned from the chromatograms in Supplementary Figure S2.2, n.d.: not detectable. 
Incubations of EGCg with tyrosinase or laccase did not result in any reaction products analysable as such by RP-UHPLC-MS. Nevertheless, EGCg had been converted by both tyrosinase and laccase. This finding was unexpected, because oxidation products of EGCg, such as theasinensins ${ }^{25}$ theacitrins ${ }^{26}$ and trimers and tetramers ${ }^{27}$ have been reported. Hence, compounds formed from EGCg might be outside the range of detection or present in too low concentrations. In literature these compounds could only be found after dedicated screening by extracted ion chromatograms or by MALDI-TOF-MS analysis. ${ }^{2,28}$ Analysis of incubations of EGCg with tyrosinase and laccase by MALDI-TOF-MS, with higher detection range, showed indeed dimer, trimer and tetramer peaks of EGCg (Sup. Fig. S2.1). As the focus of this research was on the oxidases rather than on the products formed, these compounds were not elaborated on.

No differences in type of products were found between tyrosinase and laccase; they only differed in their reaction rate on the individual catechins.

\subsubsection{Comparison of tyrosinase and laccase with respect to TF formation}

When incubating a mixture of EGC (1) and EC (2) with either tyrosinase or laccase, EGC and EC almost completely disappeared within one hour (Sup. Fig. S2.2). Peaks were annotated based on comparison of MS/MS fragmentation with published data (Table 2.1).

During the incubation with tyrosinase mainly TF (6) was formed besides some TNQ (5), which is an oxidation product of TF. The same compounds were identified in the laccase incubation, but in this case less TF and more TNQ were formed. The higher TF to TNQ ratio with tyrosinase compared to laccase suggested that $\mathrm{TF}$ is not a good substrate for tyrosinase, as was expected, because TF does not have the $o$-dihydroxylic B-ring anymore. This expectation was confirmed by oxygen consumption experiments (Sup. Fig. S2.3).

For laccase a broad spectrum of different oxidation products was expected. However, even less reaction products than with tyrosinase were found. This little molecular diversity was not expected, as other researchers found a 'Gaussian-shaped hump' in their chromatogram, caused by many different compounds with similar polarity: the so-called thearubigins. ${ }^{2,17,35}$ Explanations for missing out this molecular diversity might be the use of mushroom oxidases instead of (a mixture of) endogenous tea PPOs, and the diluted reaction conditions in the model system compared to the conditions in withering tea leaves.

An approximate mass balance was calculated for the laccase and tyrosinase incubations, based on the molar extinction coefficients of the various compounds. It appeared that $30 \%$ of the substrate was not found back as reaction products in the laccase incubation compared to $15 \%$ for tyrosinase (data not shown). This loss in compounds might partly be explained by the use of estimated molar extinction coefficients, and the formation of insoluble compounds. 
Additionally, in the tyrosinase incubation two minor peaks were formed, which were identified as catechin trimers consisting of TF and an extra EC unit (3,4). Li et al. $(2010)^{31}$ tentatively assigned a structure for this compound, which they referred to as 'compound 8 ', in which the catechin molecule is attached in a third dimension to the benzotropolone ring (Fig. 2.2). Therefore, we propose the term 'theatridimensin' (T3D) for this kind of catechin trimers, which will be used throughout this study.

Although TF cannot be oxidized by tyrosinase directly, oxidation products (TNQ and T3D) could be formed. This might be explained by coupled oxidation, where tyrosinase oxidizes an EC molecule that subsequently oxidizes or couples to a TF molecule. ${ }^{10}$

\subsubsection{Theaflavin formation with combinations of tea catechins}

Tyrosinase and laccase were also incubated with the combinations C+EGC, EC+EGCg and $\mathrm{C}+\mathrm{EGCg}$. Figure $\mathbf{2 . 3}$ shows the amounts formed per category of oxidation products. For instance, in case of the EC+EGCg incubation TFg was formed, which will be categorized as theaflavin.

The substrates in all the incubations disappeared at a similar rate (data not shown) and in all incubations theaflavins were the main initial products. Nevertheless, clear differences in the formation of oxidation products were observed. The amount of theaflavin reached in the EC+EGC incubation was approximately 4 times higher than that for the other combinations, with laccase yielding lower levels of theaflavin than tyrosinase. The same pattern applied to theanaphtoquinone formation, but in 10-20 times lower concentrations than theaflavin.

The total phenolics content also differed considerably between incubations and between enzymes. The loss in total phenolic content during tyrosinase incubation was $15 \%$ for $\mathrm{EC}+\mathrm{EGC}, \sim 65 \%$ for $\mathrm{C}+\mathrm{EGC}$ and $\mathrm{EC}+\mathrm{EGCg}$, and $96 \%$ for $\mathrm{C}+\mathrm{EGCg}$. For laccase the losses for the different incubations were $30 \%, 75 \%, 95 \%$ and $100 \%$, respectively. These results are in line with the individual catechin incubations, where tyrosinase incubations also resulted in lower losses in detectable phenolics content than laccase. This indicates that laccase has a higher activity on the oxidation products than tyrosinase. On top of that, the reactivity on oxidation products from combinations with $\mathrm{C}$ instead of $\mathrm{EC}$ appeared to be higher, as well as on oxidation products from combinations with EGCg instead of EGC. 
Altering the phenolics profile of tea with tyrosinase or laccase
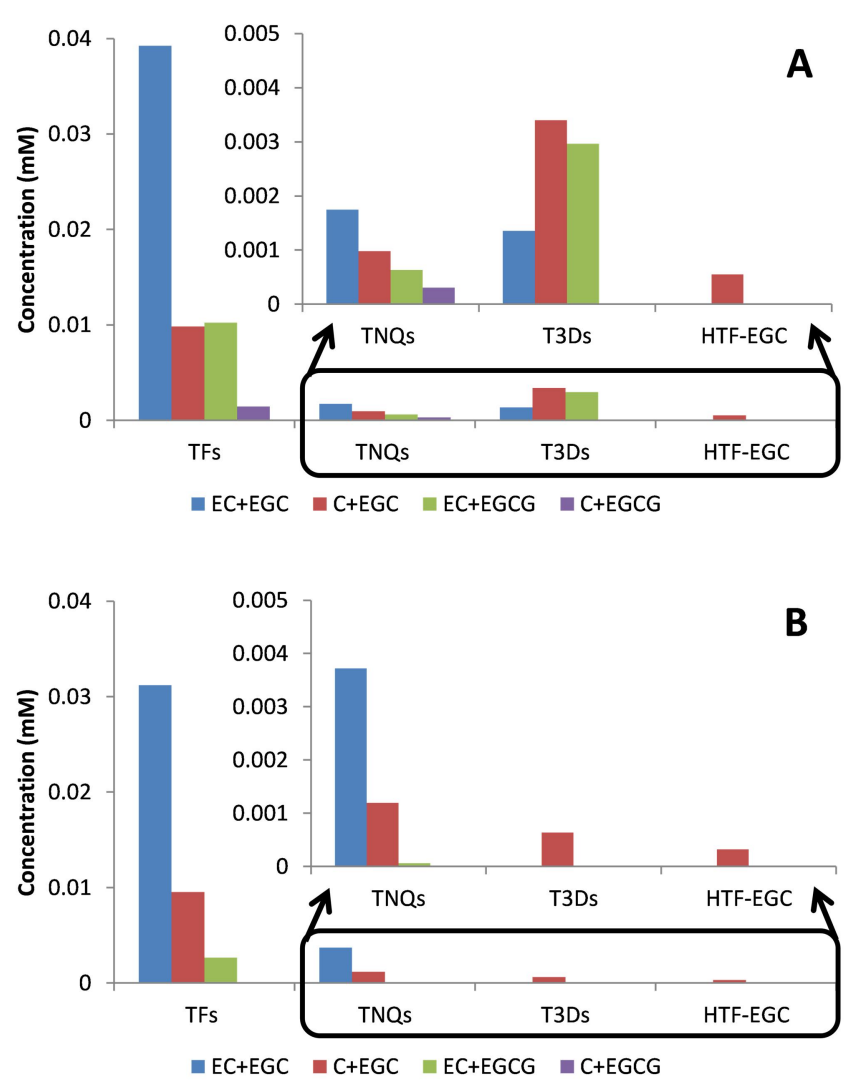

Figure 2.3 Concentration optima $(\mathrm{mM})$ of the reaction products of different catechin combinations incubated for two hours with tyrosinase $(\mathbf{A})$ or laccase $(\mathbf{B})$. The insert shows a zoom in of the minor compounds formed.

Mainly in the tyrosinase incubations the formation of theatridimensins was clearly visible, even in higher amounts than theanaphtoquinones. Besides T3D (TF-EC), the theatridimensins TF-C (stereo isomer of T3D) and tentatively T3Dg (TFg-EC) (38) were formed during incubations of $\mathrm{C}+\mathrm{EGC}$ and $\mathrm{EC}+\mathrm{EGCg}$, respectively. The formation of $\mathrm{T} 3 \mathrm{Dg}$ $(\mathrm{m} / z$ 1003) has not been reported before. Another catechin-trimer with $\mathrm{m} / \mathrm{z}$ value 1155 has been previously reported, which gives $\mathrm{m} / z 1003$ when subtracting the mass of a galloyl group $(152 \mathrm{Da}) .{ }^{27}$ Comparison of the MS/MS fragmentation patterns of this $m / z 1155$ and our $\mathrm{m} / z 1003$ compound showed two similar fragments with a neutral loss of $170 \mathrm{Da}$ (gallic acid), and $138 \mathrm{Da}$ (an RDA fragment). These fragments are common for flavanolic compounds. The MS/MS fragmentation pattern of our tentative T3Dg with $m / z 1003$ also showed fragments of $\mathrm{m} / z 617$ and $\mathrm{m} / \mathrm{z} 695$, corresponding to neutral losses of $386 \mathrm{Da}$ and $308 \mathrm{Da}$, respectively. These fragments were also found in the fragmentation pattern of T3D 
$(\mathbf{3 , 4})$, whereas these fragments were not found with compound $m / z 1155$. Moreover, the MS/MS fragmentation pattern of our tentative T3Dg showed a fragment of $m / z 865$, which is similar to the fragment of $m / z 713$ of T3D $(\mathbf{3 , 4})$, when subtracting $152 \mathrm{Da}$ for the extra galloyl group of the TFg. Finally, the compound of $\mathrm{m} / z 1155$ showed a neutral loss of 288 $\mathrm{Da}$, which corresponds to the loss of a catechin unit, whereas our compound did not show such a loss in the fragmentation pattern. Therefore, the structure of the compound with $\mathrm{m} / \mathrm{z}$ 1003 is assumed to be similar to that of T3D with an additional galloyl group at one of the catechin C-rings, T3Dg (Fig. 2.2). Theatridimensins are mainly found in tyrosinase incubations, because laccase can directly oxidize theaflavins, thereby limiting the formation of theatridimensins.

For both enzymes in the incubations of C+EGC an unknown compound with an $\mathrm{m} / \mathrm{z}$ value of 883 was found. It was assumed that it was hydroxytheaflavin (HTF) $)^{23,28}$ with an EGC coupled to it (HTF-EGC), since it equals the mass of a TF+OH+EGC. It could not be determined by MS/MS data at which position EGC is coupled to TF, whether EGC is coupled via a C-C bond between the A and C-ring or whether EGC is coupled via the benzotropolone ring in a third dimension, like the theatridimensins.

\subsubsection{Factors influencing theaflavin concentration}

The conversion of EGC+EC by tyrosinase or laccase was monitored in time (Fig. 2.4). Control samples of EGC+EC incubated without enzyme showed no changes in phenolic content (data not shown).

Figure 2.4 clearly shows that more TF was present during the tyrosinase incubation than during the laccase incubation. In the tyrosinase incubation, TF is the main oxidation product. After one hour TF slowly started to disappear, and after 120 min still $75 \%$ of TF was left. This result was expected, because tyrosinase cannot oxidize TF itself (Sup. Fig. S2.3). Only via coupled oxidation with EC-quinone and TF as nucleophile, the theanaphtoquinones and theatridimensins can be formed. In the laccase incubation, TF was at first mainly formed as well, but reached only to $80 \%$ of the amount of TF formed with tyrosinase. TF in the laccase incubation seemed to participate in follow-up reactions, as after $30 \mathrm{~min}$ it started to disappear, and after 120 min only $20 \%$ was left. This confirms that laccase catalyses reactions beyond TF. The main compound formed after TF was TNQ, but the amounts of TF and TNQ did not add up to the initial amount of catechin. As no molecular extinction coefficient has been reported for TNQ, the molar extinction coefficient of TF was used instead, which might have been a factor in the discrepancy in the catechin mass balance. Another reason might be that oxidation products were further oxidized by coupled oxidation with EC, resulting in compounds outside the range of detection. 

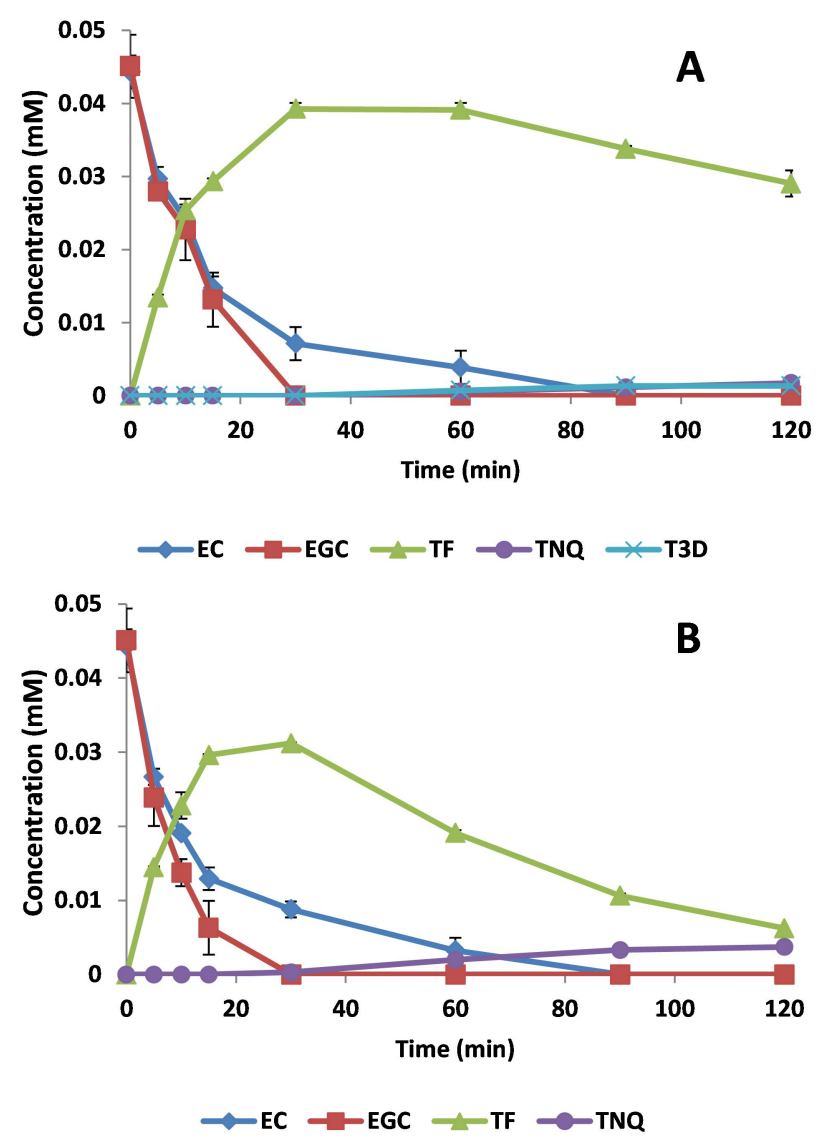

Figure 2.4 Changes in the concentrations of EC, EGC and reaction products in time upon incubation of EC+EGC with tyrosinase $(\mathbf{A})$ and laccase $(\mathbf{B})$.

To compare the influence of stereochemistry on TF formation, the EC+EGC and $\mathrm{EC}+\mathrm{EGCg}$ incubations were compared to those of $\mathrm{C}+\mathrm{EGC}$ and $\mathrm{C}+\mathrm{EGCg}$ (Fig. 2.3). Theaflavins and theanaphtoquinones were found 2 to 4 times more with EC than with $\mathrm{C}$ (neotheaflavins, 30,36). This shows that stereochemistry is very important in TF formation. This was also stated earlier, ${ }^{36}$ where the influence of stereochemistry was explained by hydrophobic association of the catechin and gallocatechin quinones to facilitate nucleophilic attack. This proceeds faster at sterically unhindered faces.

To determine the influence of a galloyl group attached to the C-ring, the incubations with EGC were compared to the incubations with EGCg. The EGC incubations gave 3 to 5 times higher concentrations of theaflavins and theanaphtoquinones in the tyrosinase incubations, and more than 10 times higher concentrations of theaflavins and 
theanaphtoquinones in the laccase incubations, compared to EGCg (Fig. 2.3). The extra galloyl group of EGCg is of influence for the formation and stability of theaflavins, also stated before. ${ }^{34}$ The galloyl group of TFg can subsequently react with another catechin, resulting in a theadibenzotropolone. ${ }^{3}$ This might explain why lower amounts of theaflavins are found, because they can react further, especially for laccase that is more active on trihydroxylic compounds than tyrosinase. However, theadibenzotropolone was not found in the incubations. This might be explained by further polymerization. If EGCg reacted with the galloyl group of TFg, the theadibenzotropolone formed has again a free galloyl group. Another catechin might subsequently react with it, in this way forming a catechin polymer. Final compounds might have fallen outside the range of detection or might have precipitated. From the experiments with the model system it can be concluded that the highest yield of theaflavins is obtained when incubating EC+EGC with tyrosinase.

\subsubsection{Theaflavin formation in green tea with exogenous oxidases}

In a model incubation with EC+EGC, tyrosinase directed the reaction towards TF production. In green tea other phenolics will be present as well. Some of these other phenolics will also be substrate for tyrosinase, which will result in a larger variety of reaction products.

Figure 2.5 shows the MS base peak chromatogram of the $24 \mathrm{~h}$ incubations of green tea with either tyrosinase, laccase or the endogenous enzymes (black tea). Peaks were annotated as described before (Table 2.1). The initial green tea extract mainly contained catechins $(\mathbf{1}, \mathbf{2}, 9,17,20,26,29)$, phenolic acid derivatives $(11,14,16,19)$ and glycosylated

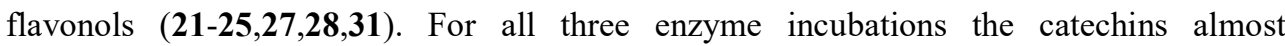
completely disappeared. For black tea, the theaflavins were the main reaction products $(\mathbf{6 , 3 3}, \mathbf{3 4}, 35)$ observed. When green tea was incubated with tyrosinase, theaflavins were the main reaction products as well, but present in concentrations three times higher $(\mathbf{6 , 3 4})$ or similar $(\mathbf{3 3}, 35)$ to black tea. For both the black tea and the tyrosinase incubation, the cumulated theaflavin content constituted approximately $70 \%$ of the total soluble phenolics. However, tyrosinase incubation yielded two times more soluble phenolics than the black tea incubation, resulting in a twice higher cumulative (absolute) theaflavin content compared to black tea. This shows the potency of tyrosinase to enrich tea in theaflavins. For the laccase incubation no theaflavins could be found. 


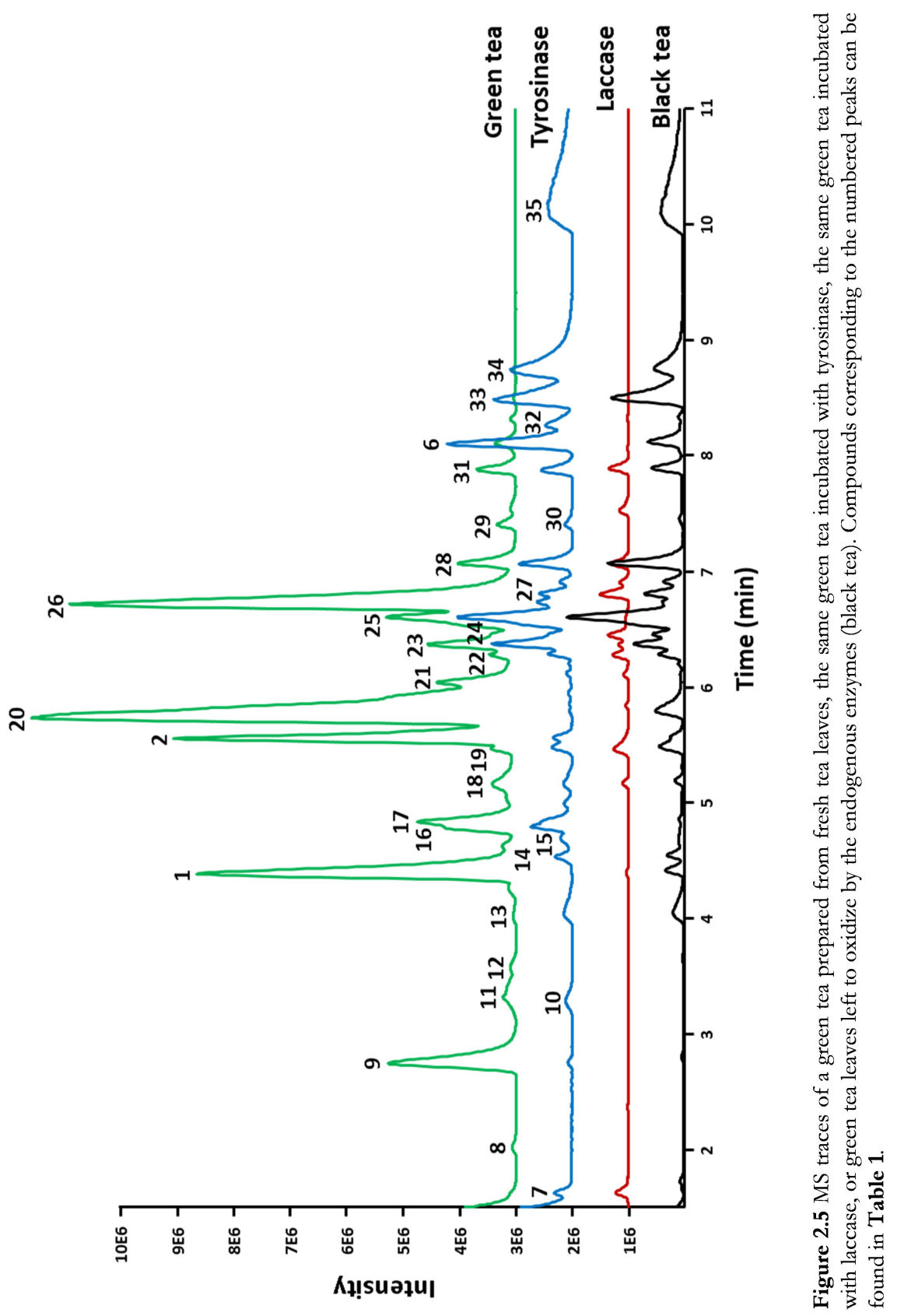


Besides theaflavins, also theacitrin gallate (13) was present in black tea. Most of the phenolic acid derivatives had reacted, whereas the glycosylated flavonols did not. When the green tea extract was incubated with tyrosinase, theacitrin gallates were formed $(\mathbf{1 0}, \mathbf{1 3}, \mathbf{1 5})$, along with dehydrotheasinensin (7) and TNQg (32). The phenolic acid derivatives content had hardly decreased and the glycosylated flavonols content had not decreased at all by tyrosinase. When the green tea extract was incubated with laccase only a phenolic acid derivative (19), dehydrotheasinensin (7) and the glycosylated flavonols were found in the incubation. Insoluble compounds were formed as well during the incubations in all samples (Sup. Fig. S2.4). These insoluble compounds were not further investigated.

\subsubsection{Opportunities for directing tea phenolics profile with exogenous oxidases}

Theaflavins are important for the quality of a black tea, especially because of their reported correlation with colour. ${ }^{6}$ The black tea prepared from the tea leaves gave the darkest brown colour. (Sup. Fig. S2.4) For the tyrosinase incubation the enrichment in theaflavins is clearly visible by their absorption maximum around $460 \mathrm{~nm} .{ }^{4}$ In the laccase incubation the absorbance increased in general, but not to a distinct brownish colour. The insoluble compounds formed, were, nevertheless, brown (Sup. Fig. S2.4).

In order to obtain a controlled oxidation to direct towards a more theaflavin-rich tea phenolics profile, tyrosinase activity is preferred over laccase activity. In black tea, prepared from the endogenous tea leaf enzymes, also oxidative enzymes other than tyrosinase will be present that can further oxidize theaflavins ${ }^{12}$ into thearubigins, hence the more brown colour. Consequently, theaflavin contents in black tea were lower than those in the tyrosinase incubation, even though all catechins had disappeared. Laccase reacts further with theaflavins and appeared less suitable for theaflavin enrichment.

Important to note is the difference in preparation conditions for the black tea versus the tyrosinase and laccase incubation of green tea. The substrate to enzyme ratio in the withering leaves will be much higher compared to the watery green tea solution incubated with enzymes. This might be the reason for the absence of thearubigins in the incubations, and hence the less brown colour of the green tea incubations.

Incubations of $\mathrm{EC}+\mathrm{EGC}$ showed that tyrosinase could very specifically produce $\mathrm{TF}$, which remained stable for an hour in the presence of tyrosinase. This concept might be applied in tea manufacturing, either by incubating green tea extract with tyrosinase, or by lowering the activity of other oxidative activities in tea leaves. 
Altering the phenolics profile of tea with tyrosinase or laccase

\subsection{References}

1. Graham, H. N. Green tea composition, consumption, and polyphenol chemistry. Preventive Medicine 1992, 21, 334-350.

2. Kuhnert, N.; Drynan, J. W.; Obuchowicz, J.; Clifford, M. N.; Witt, M. Mass spectrometric characterization of black tea thearubigins leading to an oxidative cascade hypothesis for thearubigin formation. Rapid Communications in Mass Spectrometry 2010, 24, 3387-3404.

3. Drynan, J. W.; Clifford, M. N.; Obuchowicz, J.; Kuhnert, N. The chemistry of low molecular weight black tea polyphenols. Natural Product Reports 2010, 27, 417-462.

4. Roberts, E. A. H.; Smith, R. F. Spectrophotometric measurements of theaflavins and thearubigins in black tea liquors in assessments of quality in teas. The Analyst 1961, 86, 94-98.

5. Tanaka, T.; Kouno, I. Oxidation of tea catechins: Chemical structures and reaction mechanism. Food Science and Technology Research 2003, 9, 128-133.

6. Owuor, P. O.; Obanda, M.; Nyirenda, H. E.; Mphangwe, N. I. K.; Wright, L. P.; Apostolides, Z. The relationship between some chemical parameters and sensory evaluations for plain black tea (Camellia sinensis) produced in Kenya and comparison with similar teas from Malawi and South Africa. Food Chemistry 2006, 97, 644-653.

7. Owuor, P. O.; Obanda, M. Clonal variation in the individual theaflavin levels and their impact on astringency and sensory evaluations. Food Chemistry 1995, 54, 273-277.

8. Cloughley, J. B.; Ellis, R. T. The effect of $\mathrm{pH}$ modification during fermentation on the quality parameters of central African black teas. Journal of the Science of Food and Agriculture 1980, 31, 924-934.

9. Takino, Y.; Imagawa, H.; Harikawa, H.; Tanaka, A. Studies on the mechanism of the oxidation of the catechins. Part III, Formation of a reddish-orange pigment, its spectral relationship to some benzotropolone derivatives. Agriculture, Biology and Chemistry 1964, 28, 64-71.

10. Tanaka, T.; Mine, C.; Inoue, K.; Matsuda, M.; Kouno, I. Synthesis of theaflavin from epicatechin and epigallocatechin by plant homogenates and role of epicatechin quinone in the synthesis and degradation of theaflavin. Journal of Agricultural and Food Chemistry 2002, 50, 2142-2148.

11. Mahanta, P. K.; Boruah, S. K.; Boruah, H. K.; Kalita, J. N. Changes of polyphenol oxidase and peroxidase activities pigment composition of some manufactured black teas (Camellia, sinensis L.). Journal of Agricultural and Food Chemistry 1993, 41, 272-276.

12. Gregory, R. P. F.; Bendall, D. S. The purification and some properties of the polyphenol oxidase from tea (Camellia sinensis L.). Biochemical Journal 1966, 101, 569-581.

13. Itoh, N.; Katsube, Y.; Yamamoto, K.; Nakajima, N.; Yoshida, K. Laccase-catalyzed conversion of green tea catechins in the presence of gallic acid to epitheaflagallin and epitheaflagallin 3-O-gallate. Tetrahedron 2007, 63, 9488-9492.

14. Mayer, A. M.; Staples, R. C. Laccase: New functions for an old enzyme. Phytochemistry 2002, 60, 551-565

15. Omiadze, N. T.; McHedlishvili, N. I.; Rodrigez-Lopez, J. N.; Abutidze, M. O.; Sadunishvili, T. A.; Pruidze, N. G. Biochemical processes at the stage of withering during black tea production. Applied Biochemistry and Microbiology 2014, 50, 394-397.

16. Zhao, J.; Slaga, T. J. Processing method for manufacturing black tea and an improved black tea. 2004.

17. Finger, A. In-vitro studies on the effect of polyphenol oxidase and peroxidase on the formation of polyphenolic black tea constituents. Journal of the Science of Food and Agriculture 1994, 66, 293-305.

18. Subramanian, N.; Venkatesh, P.; Ganguli, S.; Sinkar, V. P. Role of polyphenol oxidase and peroxidase in the generation of black tea theaflavins. Journal of Agricultural and Food Chemistry 1999, 47, 2571-2578. 
19. Bohin, M. C.; Vincken, J. P.; Van Der Hijden, H. T. W. M.; Gruppen, H. Efficacy of food proteins as carriers for flavonoids. Journal of Agricultural and Food Chemistry 2012, 60, 4136-4143.

20. Pelillo, M.; Cuvelier, M. E.; Biguzzi, B.; Gallina Toschi, T.; Berset, C.; Lercker, G. Calculation of the molar absorptivity of polyphenols by using liquid chromatography with diode array detection: The case of carnosic acid. Journal of Chromatography A 2004, 1023, 225-229.

21. Coxon, D. T.; Holmes, A.; Ollis, W. D.; Vora, V. C. The constitution and configuration of the theaflavin pigments of black tea. Tetrahedron Letters 1970, 11, 5237-5240.

22. Jae, K. N.; Do, Y. S.; You, J. K.; Kyung, H. S.; Yeong, S. J.; Sook, H. R.; Yokozawa, T.; Hae, Y. C. Inhibition of tyrosinase by green tea components. Life Sciences 1999, 65, PL-241-PL-246.

23. Matsuo, Y.; Tanaka, T.; Kouno, I. A new mechanism for oxidation of epigallocatechin and production of benzotropolone pigments. Tetrahedron 2006, 62, 4774-4783.

24. Guyot, S.; Vercauteren, J.; Cheynier, V. Structural determination of colourless and yellow dimers resulting from (+)-catechin coupling catalysed by grape polyphenoloxidase. Phytochemistry 1996, 42, 1279-1288.

25. Tanaka, T.; Matsuo, Y.; Kouno, I. A novel black tea pigment and two new oxidation products of epigallocatechin-3-O-gallate. Journal of Agricultural and Food Chemistry 2005, 53, 7571-7578.

26. Matsuo, Y.; Li, Y.; Watarumi, S.; Tanaka, T.; Kouno, I. Production and degradation mechanism of theacitrin $\mathrm{C}$, a black tea pigment derived from epigallocatechin-3-O-gallate via a bicyclo[3.2.1]octane-type intermediate. Tetrahedron 2011, 67, 2051-2059.

27. Yassin, G. H.; Koek, J. H.; Kuhnert, N. Identification of trimeric and tetrameric flavan-3-ol derivatives in the SII black tea thearubigin fraction of black tea using ESI-tandem and MALDI-TOF mass spectrometry. Food Research International 2014, 63, 317-327.

28. Kuhnert, N.; Clifford, M. N.; Müller, A. Oxidative cascade reactions yielding polyhydroxy-theaflavins and theacitrins in the formation of black tea thearubigins: Evidence by tandem LC-MS. Food and Function 2010, 1, 180-199.

29. Wu, C.; Xu, H.; Héritier, J.; Andlauer, W. Determination of catechins and flavonol glycosides in Chinese tea varieties. Food Chemistry 2012, 132, 144-149.

30. Lin, L. Z.; Chen, P.; Harnly, J. M. New phenolic components and chromatographic profiles of green and fermented teas. Journal of Agricultural and Food Chemistry 2008, 56, 8130-8140.

31. Li, Y.; Shibahara, A.; Matsuo, Y.; Tanaka, T.; Kouno, I. Reaction of the black tea pigment theaflavin during enzymatic oxidation of tea catechins. Journal of Natural Products 2010, 73, 33-39.

32. Dou, J.; Lee, V. S. Y.; Tzen, J. T. C.; Lee, M. R. Identification and comparison of phenolic compounds in the preparation of oolong tea manufactured by semifermentation and drying processes. Journal of Agricultural and Food Chemistry 2007, 55, 7462-7468.

33. Collier, P. D.; Bryce, T.; Mallows, R.; Thomas, P. E.; Frost, D. J.; Korver, O.; Wilkins, C. K. The theaflavins of black tea. Tetrahedron 1973, 29, 125-142.

34. Lewis, J. R.; Davis, A. L.; Cai, Y.; Davies, A. P.; Wilkins, J. P. G.; Pennington, M. Theaflavate B, isotheaflavin-3'-O-gallate and neotheaflavin-3-O-gallate: three polyphenolic pigments from black tea. Phytochemistry 1998, 49, 2511-2519.

35. Tanaka, T.; Betsumiya, Y.; Mine, C.; Kouno, I. Theanaphthoquinone, a novel pigment oxidatively derived from theaflavin during tea-fermentation. Chemical Communications 2000, 1365-1366.

36. Matsuo, Y.; Yamada, Y.; Tanaka, T.; Kouno, I. Enzymatic oxidation of gallocatechin and epigallocatechin: Effects of C-ring configuration on the reaction products. Phytochemistry 2008, 69, 3054-3061.

37. Appeldoorn, M. M.; Vincken, J. P.; Sanders, M.; Hollman, P. C. H.; Gruppen, H. Combined normal-phase and reversed-phase liquid chromatography/ESI-MS as a tool to determine the molecular diversity of A-type procyanidins in peanut skins. Journal of Agricultural and Food Chemistry 2009, 57, 6007-6013. 
Altering the phenolics profile of tea with tyrosinase or laccase

38. Ashihara, H.; Deng, W.-W.; Mullen, W.; Crozier, A. Distribution and biosynthesis of flavan-3-ols in Camellia sinensis seedlings and expression of genes encoding biosynthetic enzymes. Phytochemistry 2010, 71, 559-566.

39. Hashimoto, F.; Nonaka, G.; Nishioka, I. Tannins and related compounds. LXIX. Isolation and structure elucidation of B,B'-linked bisflavanoids, theasinensins D-G and oolongtheanin from oolong tea. (2). Chemical \& Pharmaceutical Bulletin 1988, 36, 1676-1684. 


\subsection{Supplementary data}

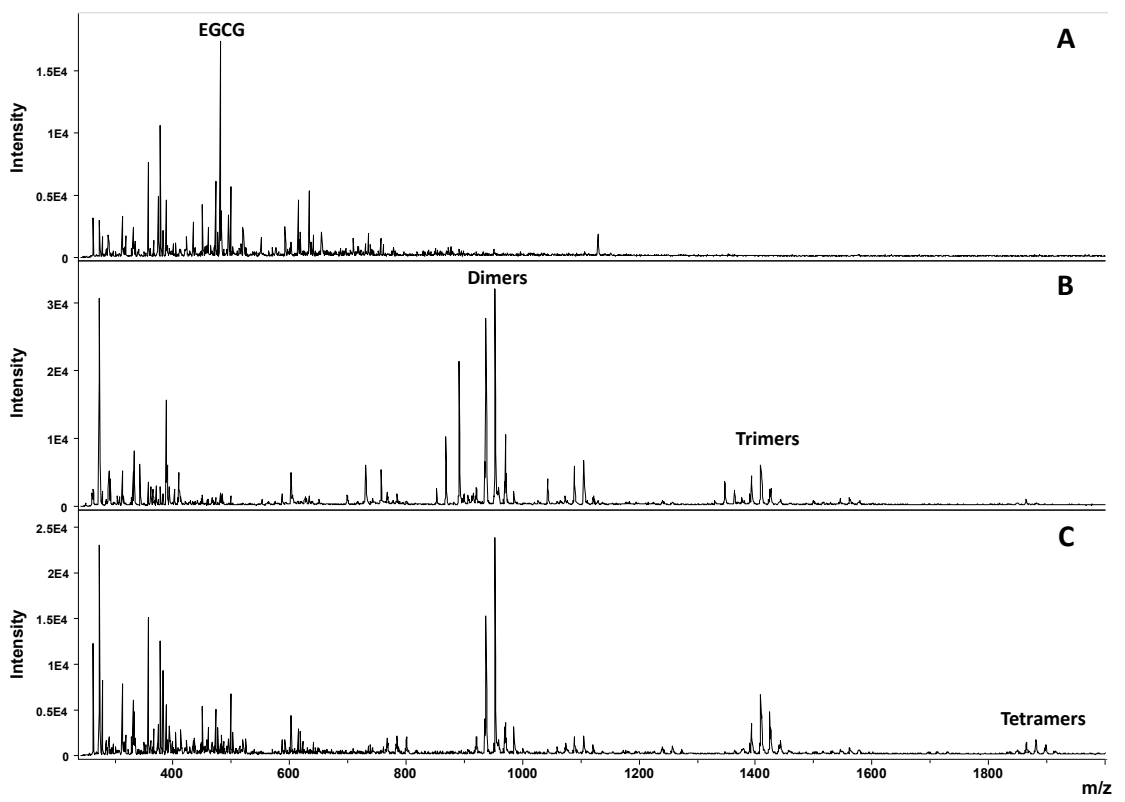

Figure S2.1 MALDI-TOF-MS spectrum of A) Control EGCg, B) EGCg incubated with tyrosinase and C) EGCg incubated with laccase.

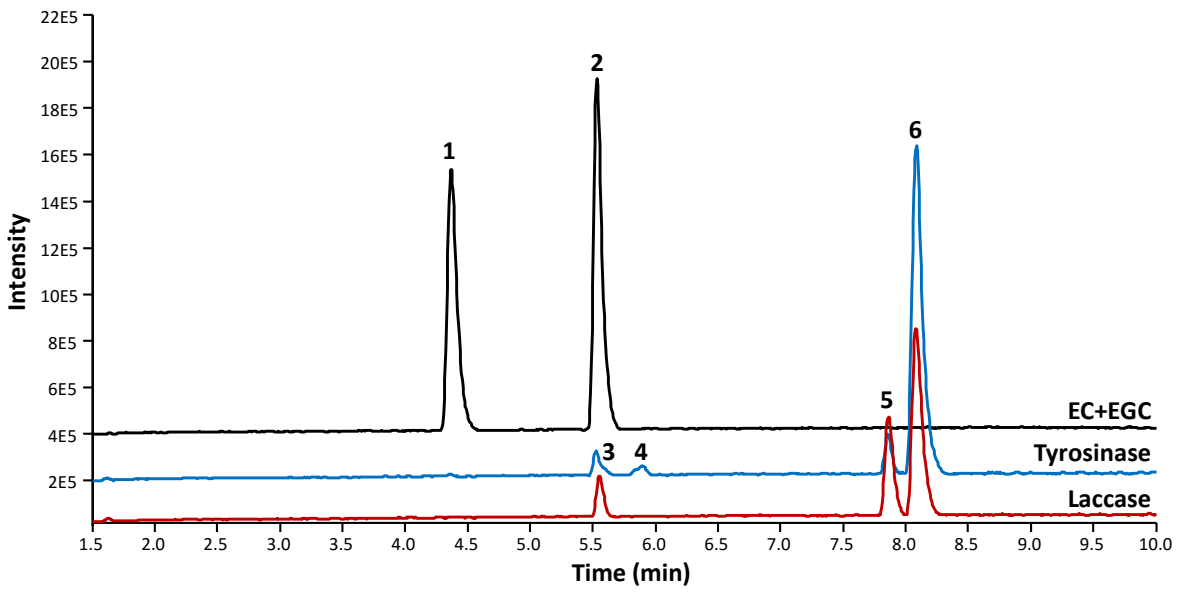

Figure S2.2 MS trace of a one hour EC+EGC incubation using tyrosinase or laccase. The compounds corresponding to the numbered peaks can be found in Table 2.1. 
Altering the phenolics profile of tea with tyrosinase or laccase

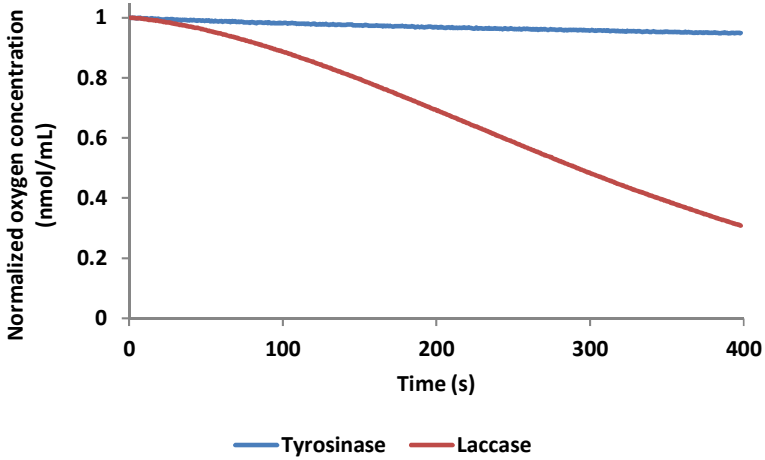

Figure S2.3 Oxygen concentration $(\mathrm{nmol} / \mathrm{mL})$ in time $(\mathrm{s})$ in an incubation of theaflavin-3-gallate with either tyrosinase or laccase.

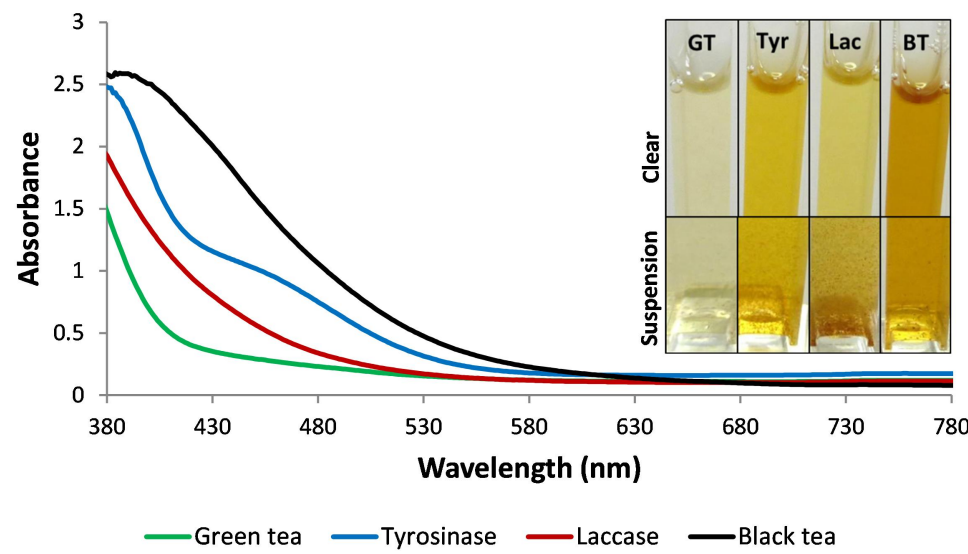

Figure S2.4 Absorbance spectra from $380 \mathrm{~nm}$ to $780 \mathrm{~nm}$ of a green tea extract, a green tea extract incubated with tyrosinase, a green tea extract incubated with laccase, and a black tea extract prepared from the same green tea leaves. The inset shows the clear solutions and the suspension formed after these incubations. 
Chapter 2 


\title{
Chapter 3
}

\section{An MS/MS method based on selected ions detects low abundance phenolics in black tea - theatridimensins as product of the oxidative cascade}

\begin{abstract}
Three rapid MS screening methods were compared for their ability to annotate two-stepoxidation products in black teas without the need of prior fractionation: (i) full MS, (ii) MS/MS on selected ions, and (iii) selected reaction monitoring (SRM), the first two in combination with post analysis extracted ion chromatography (EIC). A model system of theaflavin (TF), epicatechin (EC) and tyrosinase was used to prepare the two-step-oxidation product theatridimensin (T3D), consisting of three oligomerized catechin subunits. The $\mathrm{MS}^{2}$ fragmentation pattern of T3D was compared with that of an isomeric catechin trimer from black tea, TFsEC. MS ${ }^{2}$ signature fragments were found to distinguish the two isomers, i.e. $m / z 617$ for T3D and $m / z 563$ for TFsEC. When exploring black tea for the occurrence of such two-step-oxidation products, the use of MS/MS on selected ions combined with EIC proved to be the most suitable. It not only enabled monitoring $\mathrm{MS}^{2}$ of compounds present in low abundance, it also provided a complete $\mathrm{MS}^{2}$ data set, facilitating the discovery of other isomers, for example theaflavate A. The occurrence of T3Ds and T3Dgs in various black teas was shown for the first time and the 'oxidative cascade hypothesis' was extended with novel oxidation products.
\end{abstract}

Based on: Annewieke J.W. Verloop, Jean-Paul Vincken, Harry Gruppen. 2016 - Submitted for publication. 


\subsection{Introduction}

In plant-derived food products mixtures of phenolics are widespread, the complexity of which is often increased by processing. The manufacturing of black tea is a well-known example. For quality control, ${ }^{1}$ geographical traceability, ${ }^{2}$ authentication ${ }^{3-5}$ and studying effects of processing methods, ${ }^{6}$ it is important to provide detailed compositional analysis of the tea phenolics present, including those present in low abundance.

When green tea leaves are processed into black tea, the green leaf phenolics are oxidized into more complex phenolics by the leaves' endogenous oxidative enzymes. The main phenolic compounds in green tea are catechins (also referred to as flavan-3-ols), characterized by their meta-5,7-dihydroxy substituted A-ring and di- or trihydroxylic Bring. These catechins form a set of precursors with limited molecular diversity, which after oxidation results in the around 10,000 different, complex phenolics in black tea, also referred to as thearubigins. ${ }^{7}$ Their formation is explained by the 'oxidative cascade hypothesis' introduced in 2010. ${ }^{7}$ The 'oxidative cascade hypothesis' starts with oligomerization of the catechin subunits, yielding four types of interflavanic configuration: the theaflavin (TF) type, the theasinensin (TS) type, the theacitrin (TC) type and the theanaphtoquinone (TNQ) type. The next step is hydroxylation of the catechin oligomers. Hydroxyl groups can be added to all unsubstituted carbons of the aromatic rings in the oligomers via nucleophilic attack.

To understand the oligomerization reactions that occur in the formation of black tea, a model system has recently been used with only a limited number of precursors, TF with epicatechin (EC), and a single oxidase with well-defined activity, tyrosinase. ${ }^{8} \mathrm{TF}$ is the oligomerization product of EC and epigallocatechin. In the presence of EC-quinone, formed by the enzymatic oxidation of EC, TF can be transformed into at least three different products (Fig. 3.1). Upon coupled oxidation, ${ }^{9-11}$ a redox-reaction occurs in which the ECquinone reduces back to EC while oxidizing TF into TNQ, a dimeric flavonoid in which the EC-quinone is not incorporated. TNQ is considered an oligomerization product in the 'oxidative cascade hypothesis'. The formation of TNQ, however, needs two oxidation steps: an oligomerization into TF, and a rearrangement of TF into TNQ. Other two-stepoxidation products via TF are formed by a second oligomerization step, when EC-quinone via its B-ring couples to the benzotropolone ring of $\mathrm{TF}$, yielding the trimeric catechin theatridimensin (T3D). ${ }^{8,12}$ Alternatively, the EC-quinone B-ring can attack the aromatic ring in the interflavanic configuration of TF, upon which a TS-type cross-link is formed, yielding the trimeric catechin TFsEC (the ' $\mathrm{s}$ ' indicating the TS-type of oligomerization). ${ }^{13}$ These three different two-step-oxidation products formed from TF indicate that the oligomerization level in the 'oxidative cascade hypothesis' requires further refinement. 


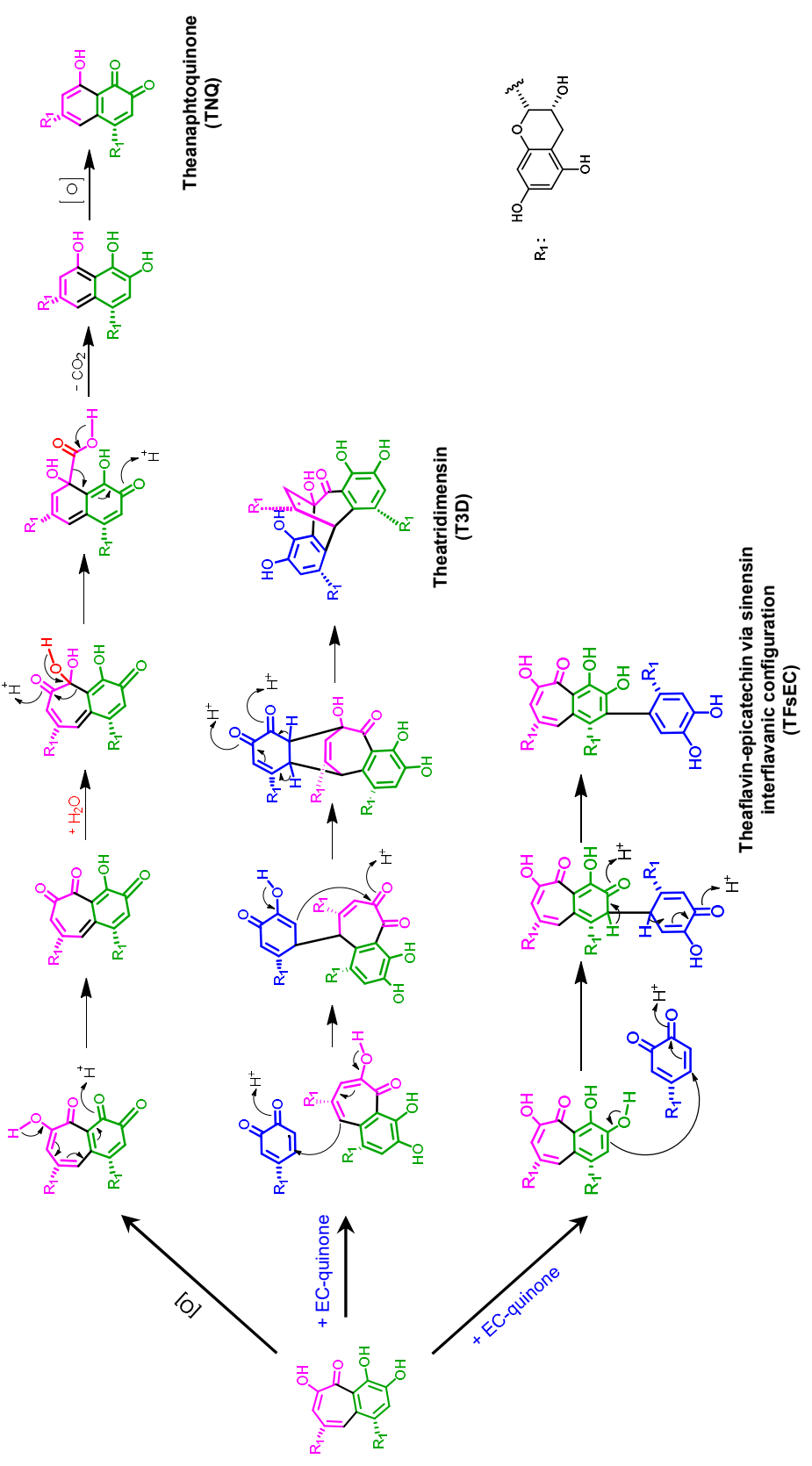


Characterization of the different oxidation products from catechins is necessary to understand the oxidative processes resulting in black tea. Often this characterization is done by NMR spectroscopy after extensive, laborious purification steps. ${ }^{14}$ Hence, there is a need for rapid screening methods to annotate compounds in complex mixtures without extensive sample preparation, e.g. LC-MS. Extracted ion chromatograms (EIC) of specific $m / z$ values upon mass spectrometric analysis have been used for this purpose. ${ }^{15}$ Main disadvantages of this approach are (i) low sensitivity, as mainly highly abundant compounds will be measured, and (ii) lack of $\mathrm{MS}^{2}$ data, for the same reason., ${ }^{713}$

The aim of the present research is to select a rapid screening method to annotate the various two-step-oxidation products in black teas, without the need of prior fractionation of the sample. Three MS modes, sometimes combined with EIC, were compared: (i) full MS, (ii) MS/MS on selected ions, and (iii) selected reaction monitoring (SRM). T3Ds and TFsECs were used as examples of isomeric two-step-oxidation products to be analysed, as their occurrence in tea is expected. Seven teas were screened for the presence of these two isomers. It is hypothesized that with specific MS settings, like SRM or MS/MS on selected ions, $\mathrm{MS}^{2}$ data can also be obtained for compounds with low abundance. By deriving signature fragments from the $\mathrm{MS}^{2}$ data, two-step-oxidation products might be tentatively annotated and distinguished from isomers.

\subsection{Materials and Methods}

\subsubsection{Materials}

(-)-Epicatechin (EC), theaflavin-3-gallate (TFg), L-ascorbic acid and mushroom tyrosinase were purchased from Sigma Aldrich (St. Louis, MO, USA). Anhydrous citric acid was purchased from Acros Organics (Geel, Belgium) and disodium hydrogen phosphate from Merck (Darmstadt, Germany). (-)-Epigallocatechin (EGC) was prepared from EGCg (Sigma Aldrich) as described elsewhere. ${ }^{16}$ Ultra-high-performance liquid chromatography mass spectrometry (UHPLC-MS) grade acetonitrile (ACN) and methanol $(\mathrm{MeOH})$ were obtained from Biosolve (Valkenswaard, The Netherlands). Water was prepared using a Milli-Q water purification system (Millipore, Billerica, MA, USA). Various teas from different origin were obtained from a local store: Darjeeling First Flush (India), Darjeeling Second Flush (India), Nepal First Flush (Nepal), Keemun Congou (Anhui, China), China black Yunnan (Yunnan, China), Oolong Finest Taiwan (Taiwan), Pu Ehr (China), and Lapsang Souchong (China). 
MS/MS on selected ions to detect low abundance phenolics in black tea

\subsubsection{Preparation of theaflavin pool}

Theaflavin (TF) was prepared by incubation of a mixture of EC and EGC with tyrosinase. EC and EGC were used at a concentration of $0.5 \mathrm{mM}$ each. Tyrosinase was added to a final concentration of $0.001 \mathrm{U} / \mathrm{mL}$ (one unit equalling the amount of enzyme that consumes $1 \mu \mathrm{mol}$ of oxygen per min for oxidation of catechin at $\mathrm{pH} 5.5$ and $25{ }^{\circ} \mathrm{C}$ ). Incubations were performed in citric acid - sodium phosphate buffer $(30 \mathrm{mM}) \mathrm{pH} 5.5$. The reaction flask was incubated in the dark at $25^{\circ} \mathrm{C}$ under continuous stirring. After one hour the reaction was stopped by adding $20 \mu \mathrm{L}$ of saturated ascorbic acid solution per $\mathrm{mL}$ reaction mixture. The amount of ascorbic acid added was sufficient to arrest the reaction for at least $12 \mathrm{~h}$. Subsequently, the sample was subjected to solid-phase extraction (SPE) using a $10 \mathrm{~g} \mathrm{C} 18$ Sep-Pak column washed and eluted with water and $\mathrm{MeOH}$ according to the instructions of the manufacturer (Waters, Milford, MA, USA). The $\mathrm{MeOH}$ fraction was evaporated under reduced pressure, dissolved in water and freeze-dried. The freeze-dried material was dissolved in water to a concentration of $\sim 3 \mathrm{mg} / \mathrm{mL}$. Subsequently, it was purified by Flash chromatography, using a $4 \mathrm{~g}$ Reveleris $\mathrm{C} 18$ column on a Reveleris Flash system (Grace, Deerfield, IL, USA) operated at $15 \mathrm{~mL} / \mathrm{min}$. Water containing $1 \%(\mathrm{v} / \mathrm{v})$ $\mathrm{ACN}$ and $0.1 \%(\mathrm{v} / \mathrm{v})$ acetic acid (eluent $\mathrm{A})$ and $\mathrm{ACN}$ containing $0.1 \%(\mathrm{v} / \mathrm{v})$ acetic acid (eluent $B$ ) were used as eluents. The following elution profile was used: 0-1 min, isocratic on $0 \% \mathrm{~B} ; 1-11 \mathrm{~min}$, linear gradient from $0-50 \% \mathrm{~B} ; 11-13 \mathrm{~min}$, linear gradient from 50 $100 \% \mathrm{~B} ; 13-16 \mathrm{~min}$, isocratic on $100 \% \mathrm{~B}$. Fractions were collected and analysed by RPUHPLC-MS as described in paragraphs 2.5 and 2.6. Fractions were pooled to yield a $95 \%$ pure TF pool, annotation was based on MS data, whereas quantification was based on UV absorbance at $270 \mathrm{~nm}$. The TF pool was freeze-dried and stored at $-20{ }^{\circ} \mathrm{C}$. During the preparation steps, the sample was kept from light.

\subsubsection{Oxidation of TF or TFg using EC and tyrosinase}

EC was incubated in equal molarity with either TF or TFg at a total phenolics concentration of $0.1 \mathrm{mM}$. Tyrosinase was added to a final concentration of $0.0001 \mathrm{U} / \mathrm{mL}$. Incubations were performed in citric acid - sodium phosphate buffer $(30 \mathrm{mM}) \mathrm{pH} 5.5$. Samples $(1 \mathrm{~mL})$ were incubated at $25^{\circ} \mathrm{C}$ for $60 \mathrm{~min}$, after which the reaction was stopped by adding $20 \mu \mathrm{L}$ of freshly prepared saturated ascorbic acid solution. The samples were centrifuged $\left(10,000 \mathrm{~g}, 5 \mathrm{~min}, 20{ }^{\circ} \mathrm{C}\right)$ prior to analysis by reversed-phase liquid chromatography. Compounds were tentatively annotated and semi-quantified by mass spectrometry. 


\subsubsection{Extraction of black tea phenolics}

Black tea $(0.5 \mathrm{~g})$ was three times extracted with $50 \mathrm{~mL}$ boiling water for $10 \mathrm{~min}$ under continuous stirring. Extracts were filtered through paper filter (Grade 1, Whatman, Chalfont St. Giles, UK) by Büchner filtration and the filtrates were combined. The samples were centrifuged $\left(10,000 \mathrm{~g}, 5 \mathrm{~min}, 20^{\circ} \mathrm{C}\right)$ prior to analysis by reversed-phase liquid chromatography.

\subsubsection{RP-UHPLC analysis}

Samples were analysed on an Accela UHPLC system (Thermo Scientific, San Jose, CA, USA) equipped with a pump, an auto sampler at $15{ }^{\circ} \mathrm{C}$ and a photodiode array (PDA) detector. Samples $(1 \mu \mathrm{L})$ were injected onto a Hypersil Gold column $(2.1 \times 150 \mathrm{~mm}$, particle size of $1.9 \mu \mathrm{m}$, Thermo Scientific). Water containing $1 \%(\mathrm{v} / \mathrm{v}) \mathrm{ACN}$ and $0.1 \%(\mathrm{v} / \mathrm{v})$ acetic acid (eluent A) and ACN containing $0.1 \%(\mathrm{v} / \mathrm{v})$ acetic acid (eluent B) were used as eluents. The flow rate was $300 \mu \mathrm{L} / \mathrm{min}$, and the column oven temperature was controlled at $30{ }^{\circ} \mathrm{C}$. The PDA detector was set to measure $200-600 \mathrm{~nm}$. The following elution profile was used: 0-1 min, isocratic on $5 \%(\mathrm{v} / \mathrm{v}) \mathrm{B} ; 1-12 \mathrm{~min}$, linear gradient from $5-50 \%(\mathrm{v} / \mathrm{v}) \mathrm{B}$; 12-13 min, linear gradient from $50-100 \%$; $13-18 \mathrm{~min}$, isocratic on $100 \%(\mathrm{v} / \mathrm{v}) \mathrm{B} ; 18-18.1$ min, linear gradient from $100-5 \%(\mathrm{v} / \mathrm{v}) \mathrm{B}$; and 18.1-22 min, isocratic on 5\% (v/v) B.

\subsubsection{Electrospray Ionization - Mass Spectrometry (ESI-MS)}

Mass spectrometric data were obtained on a Velos Pro mass spectrometer (Thermo Scientific) equipped with an ESI probe coupled to the RP-UHPLC system. Nitrogen was used as sheath gas and auxiliary gas. Data were collected over the $\mathrm{m} / \mathrm{z}$ range of 250-2000. Data-dependent $\mathrm{MS}^{\mathrm{n}}$ analysis was performed with a normalized collision energy of $35 \%$. The $\mathrm{MS}^{\mathrm{n}}$ fragmentation was performed on the most intense product ion in the $\mathrm{MS}^{\mathrm{n}-1}$ spectrum, with wideband activation to exclude fragments with neutral losses of $18 \mathrm{Da}$ $\left(\mathrm{H}_{2} \mathrm{O}\right)$, and with a dynamic exclusion for $5 \mathrm{~s}$, when the most intense product ion appeared twice within $5 \mathrm{~s}$. The system was tuned with theaflavin-3-gallate in negative ionization mode. Most settings were optimized via automatic tuning using "Tune Plus" (Xcalibur 2.07, Thermo Scientific). The transfer tube temperature was $350{ }^{\circ} \mathrm{C}$, and the source voltage was $4.0 \mathrm{kV}$. Data acquisition and reprocessing were performed with Xcalibur 2.07 (Thermo Scientific). The MS was run in three different modes: Full MS, MS/MS on selected ions, and selected reaction monitoring (SRM). With MS/MS on selected ions, the MS will only monitor the $\mathrm{MS}^{2}$ data of the parent ions specified in the MS/MS inclusion list, during a specified timeframe. With SRM, not only the parent ion is specified, but also the fragment of that ion. Only this specified fragment in the $\mathrm{MS}^{2}$ will be monitored by the MS. 
Compounds were quantified by MS signal relative to $\mathrm{TFg}$, and the amounts were expressed as TFg equivalents. The TFg calibration curve (ranging from 0.0006-0.004 mM) had a correlation coefficient of 0.99 .

\subsection{7 $\mathrm{MS}^{2}$ fragment data extrapolation for TFsEC}

$\mathrm{The} \mathrm{MS}^{2}$ data of TFsEC was extrapolated from that of the digallated form of TFsEC, i.e. TFggsEC $\left(m / z\right.$ 1155), ${ }^{13}$ under the assumption that fragmentation of TFsEC during $\mathrm{MS}^{2}$ occurs at the same linkages as in TFggsEC, except for the linkages connecting the gallic acids. The main fragment ions of TFggsEC were $\mathrm{m} / \mathrm{z} 867, \mathrm{~m} / \mathrm{z} 1137, \mathrm{~m} / \mathrm{z} 1003, \mathrm{~m} / \mathrm{z} 985$ and $m / z$ 1017. The fragments of $m / z 1003$ and $m / z 985$ resulted from cleavage of the gallic acid group and were not further taken into account for TFsEC. The other three fragments $\mathrm{m} / z$ 867, $\mathrm{m} / \mathrm{z} 1137$ and $\mathrm{m} / \mathrm{z} 1017$ were extrapolated to TFsEC by subtracting the mass of the two gallic acids (304 Da), yielding $\mathrm{m} / \mathrm{z} 563, \mathrm{~m} / \mathrm{z} 713$ and $\mathrm{m} / \mathrm{z} 833$ for TFsEC, respectively. Supplemental Figure S3.1 shows the theoretical fragmentation pattern of TFsEC. ${ }^{13}$

\subsection{Results}

\subsubsection{Signature fragments of oxidation products from theaflavins}

The aim of the present research was to select the best LC-MS screening method to annotate phenolics present in low abundance in complex mixtures as black tea, without preparative fractionation. For this selection, the two-step-oxidation products T3Ds and TFsECs were used as representatives of isomeric phenolic compounds expected to be present in low abundance in black tea. To enable annotation of compounds in low abundance from a complex mixture with $\mathrm{MS}, \mathrm{MS}^{2}$ signature fragments should be known. To obtain T3Ds, an incubation of either TF or TFg in the presence of EC and tyrosinase was performed. ${ }^{8}$ Figures 3.2A and 3.2C show the MS base peak trace of these incubations, after one hour of oxidation of TF or TFg, respectively. Peaks were tentatively annotated based on comparison of MS/MS fragmentation patterns with published data (Table 3.1).

Upon oxidation of TF (3), different T3Ds (4-6) were formed besides TNQ (2). The T3D yield was divided over different stereoisomers, which resulted in higher $\mathrm{s} / \mathrm{n}$ ratios. Therefore, the T3Ds $(m / z$ 851) were not clearly visible in the MS base peak chromatogram (Fig. 3.2A). They were found when obtaining an extracted ion chromatogram (EIC) of $\mathrm{m} / z$ 851 (Fig. 3.2B). Upon oxidation of TFg (10), T3Dgs $(\mathbf{7}, \mathbf{8})$ and TNQg $(\mathbf{9})$ were formed (Fig. 3.2C). 


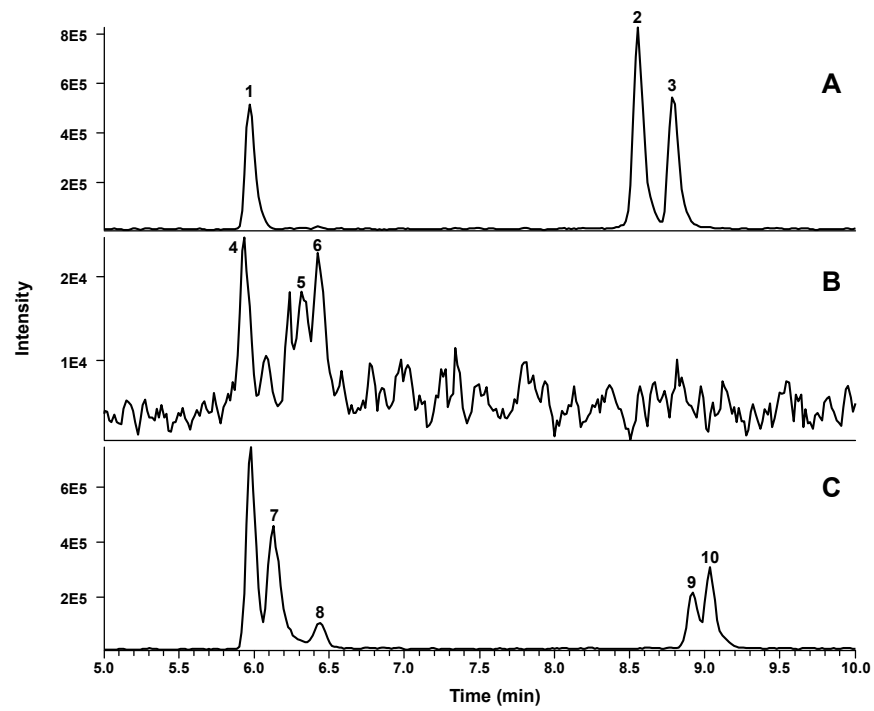

Figure 3.2 MS base peak trace of a $1 \mathrm{~h}$ incubation of TF+EC $(\mathbf{A})$ and TFg+EC (C) with tyrosinase. An EIC of $m / z, 851$ from the MS base peak of the TF+EC incubation (B). The compounds corresponding to the numbered peaks are listed in Table 3.1.

Table 3.1 Compounds found by RP-UHPLC-MS $\mathrm{n}$ from the TF+EC and TFg+EC incubations using tyrosinase.

\begin{tabular}{|c|c|c|c|c|c|}
\hline $\begin{array}{c}\text { Peak } \\
\text { no. }\end{array}$ & $\begin{array}{c}\text { Retention } \\
\text { time } \\
\text { (min) }\end{array}$ & $\begin{array}{c}{[\mathrm{M}-\mathrm{H}]^{-}} \\
(m / z)\end{array}$ & $\begin{array}{c}\mathrm{MS}^{2} \text { fragments } \\
\qquad(m / z)\end{array}$ & $\begin{array}{l}\lambda_{\max }^{*} \\
(\mathbf{n m})\end{array}$ & Tentative annotation \\
\hline 1 & 5.97 & 289 & 245,205 & 276 & Epicatechin $^{17}$ \\
\hline 2 & 8.56 & 533 & $505,471,349,305$ & $252,278,438$ & Theanaphtoquinone ${ }^{18}$ \\
\hline 3 & 8.78 & 563 & $407,379,425,241$ & $266,375,452$ & Theaflavin $^{19}$ \\
\hline 4 & 5.92 & 851 & $713,617,815,695,707$ & 271,381 & Theatridimensin ${ }^{12}$ \\
\hline 5 & 6.23 & 851 & $617,713,815,707$ & 270,381 & Theatridimensin ${ }^{12}$ \\
\hline 6 & 6.43 & 851 & $815,683,807,713,617$ & 263,382 & Theatridimensin ${ }^{12}$ \\
\hline 7 & 6.13 & 1003 & $833,617,695,865$ & 278 & Theatridimensin gallate ${ }^{8}$ \\
\hline 8 & 6.44 & 1003 & $833,617,695,865$ & 278 & Theatridimensin gallate $^{8}$ \\
\hline 9 & 8.92 & 685 & $559,519,515,507$ & 278 & Theanaphtoquinone gallate ${ }^{20}$ \\
\hline 10 & 9.03 & 715 & $563,527,545$ & 272,382 & Theaflavin gallate $^{19}$ \\
\hline
\end{tabular}

${ }^{*}$ Only $\lambda_{\max }$ values above $250 \mathrm{~nm}$ are shown. 
Figure 3.3 shows the $\mathrm{MS}^{2}$ spectrum of T3D $(\mathrm{m} / z$ 851), together with its fragmentation routes. The three main fragment ions of T3D were $\mathrm{m} / \mathrm{z} 617, \mathrm{~m} / \mathrm{z} 713$ and $\mathrm{m} / \mathrm{z} 815$. The latter originated after a neutral loss of $36 \mathrm{Da}$, a loss consistent with two water molecules. Fragment $\mathrm{m} / \mathrm{z} 713$ originated after a neutral loss of $138 \mathrm{Da}$, indicating a retro-Diels-Alder (RDA)-type fragmentation. Fragment $\mathrm{m} / \mathrm{z} 617$ likely resulted from the cleavage of three bonds in the tripartite interflavanic configuration of the benzotropolone ring of T3D. As this interflavanic configuration is typical for T3Ds ${ }^{12}$, this fragment of $m / z 617$ was used as the signature fragment of T3D.

The fragments of TFsEC were extrapolated from literature, as described in subchapter 3.2.7. Fragment $m / z 833$ originated after a neutral loss of $18 \mathrm{Da}$, a loss consistent with one water molecule (no wideband activation setting was used). Fragment $\mathrm{m} / \mathrm{z} 713$ originated after a neutral loss of $138 \mathrm{Da}$, indicating an RDA-type fragmentation. Fragment $\mathrm{m} / \mathrm{z} 563$ originated after a neutral loss of $288 \mathrm{Da}$, which represents the loss of a catechin moiety. This latter fragmentation is not likely to occur for T3Ds, because there the extra catechin is doubly linked to the TF benzotropolone ring in the T3D interflavanic configuration. Therefore, this fragment of $\mathrm{m} / \mathrm{z} 563$ can be used as a signature fragment for TFsEC, to distinguish this isomer from T3D.

\subsubsection{Comparison of MS screening methods}

Different settings in the mass spectrometric analysis were used to screen for minor phenolic compounds in Chinese black Yunnan tea. The outcomes with the different settings, both during analysis and post-analysis, are compared with each other in Figure 3.4, where T3Ds and TFsECs are screened for as representatives of isomeric two-stepoxidation products. Figure 3.4A shows the full MS chromatogram of a black tea (Chinese black Yunnan). Around 30 peaks could be distinguished, of which 6 non-oxidized catechins and $4 \mathrm{TFs}$, as indicated in the chromatogram. Figure 3.4B shows the EIC of $\mathrm{m} / \mathrm{z} 851$, corresponding to T3D and/or TFsEC, obtained during post-analysis from the full MS data set. This EIC chromatogram contained two main peaks, the intensity of which was 100 times lower than those of the main peaks in the full MS chromatogram (Fig. 3.4A). Consequently, $\mathrm{MS}^{2}$ data were not recorded for $m / z 851$.

Alternative to post-analysis processing of the data, the compounds of interest could also be specifically screened for during an MS procedure in which MS/MS is performed on $a$ priori selected $\left(\mathrm{MS}^{1}\right)$ parent ions. The data recorded comprised all $\mathrm{MS}^{2}$ fragments of the $a$ priori selected parent ions. Figure 3.4C shows the $\mathrm{MS}^{2}$ chromatogram of the a priori selected ion $\mathrm{m} / \mathrm{z}$ 851. Three peaks with parent mass $\mathrm{m} / \mathrm{z} 851$ could be distinguished and their $\mathrm{MS}^{2}$ data were recorded. Two of these isomer peaks were not found in the EIC of the full MS chromatogram; they had a main $\mathrm{MS}^{2}$ fragment of $m / z 617$, the signature fragment 
of T3D. The other isomer peak in Figure 3.4C was already detected in the EIC of the full MS, but now the $\mathrm{MS}^{2}$ data were obtained as well. It showed a main $\mathrm{MS}^{2}$ fragment of $\mathrm{m} / z$ 579, which is neither a fragment of T3D nor of TFsEC. The compound was annotated as theaflavate $\mathrm{A}_{,}^{21,22}$ a catechin dimer that has a TF-type interflavanic configuration via the gallic acid ester group (Sup. Fig. S3.2). The peak found in the EIC of the full MS at retention time $8 \mathrm{~min}$ (Fig. 3.4B), was not recovered in Figure 3.4C.

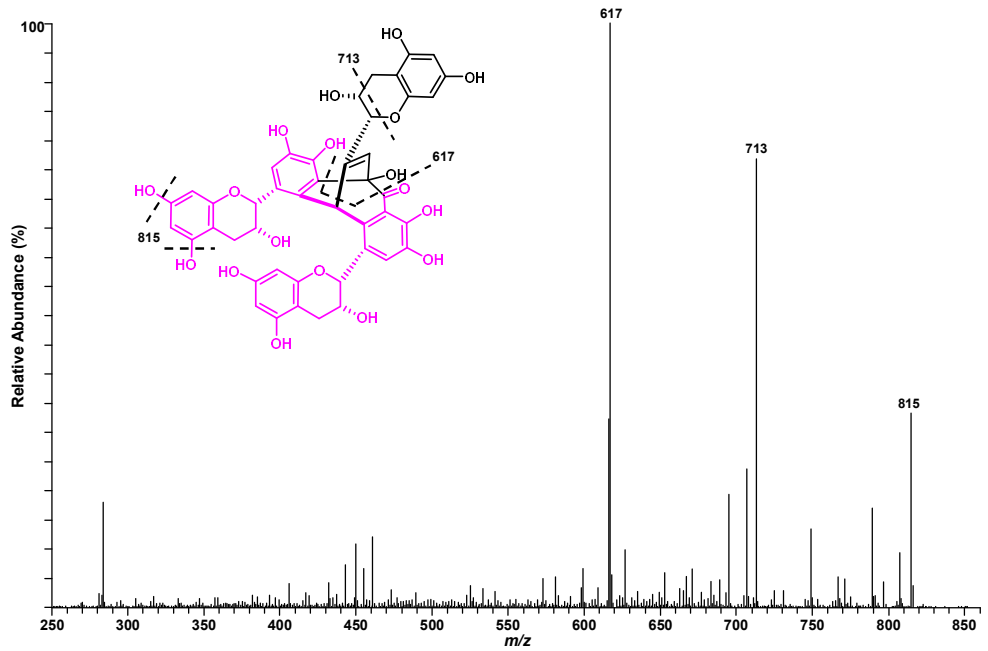

Figure 3.3 $\mathrm{MS}^{2}$ fragmentation pattern of theatridimensin, $\mathrm{m} / \mathrm{z}, 851$, including a schematic representation of the main fragmentation routes. The signature fragment with $m / z .617$ is shown in pink.

After MS/MS on the selected ion, a post-analysis EIC can be made with the signature fragment of the compounds of interest, as shown in Figures 3.4D and 3.4F for T3Ds $(\mathrm{m} / z$ $617)$ and for TFsECs $(m / z 563)$, respectively.

Alternatively, MS was performed in selected reaction monitoring (SRM) mode, where both the parent ion and the $\mathrm{MS}^{2}$ fragment ion were a priori selected. Subsequently, only the selected $\mathrm{MS}^{2}$ value from the selected parent ion was recorded. The data gathered only showed the information of the EIC from the MS/MS data on selected ions, as can be seen when comparing the SRM of T3D (Fig. 3.4E) with the EIC of T3D from the MS/MS data on selected ions (Fig. 3.4D), and the SRM of the TFsEC (Fig. 3.4G) with the EIC of TFsEC from the MS/MS data on selected ions (Fig. 3.4F). Both the MS/MS on selected ions with post-analysis EIC and the SRM method could detect the expected $\mathrm{MS}^{2}$ fragments of two-step-oxidation products in a black tea. 


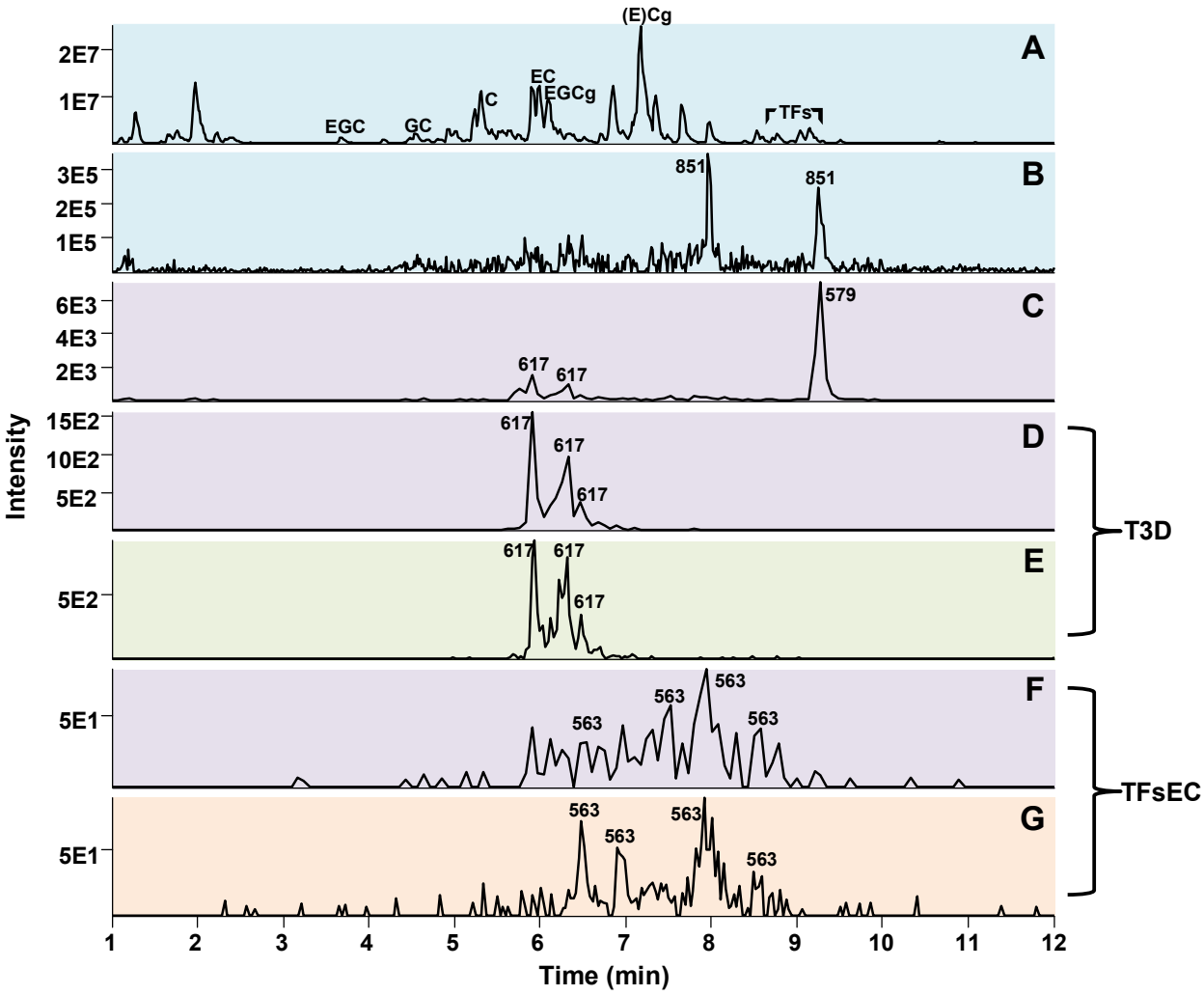

Figure 3.4 RP-UHPLC-MS profiles from a Chinese black Yunnan tea. A) Full MS, B) EIC of $m / z$. 851 from full MS data, C) MS/MS on selected ion $m / z 851$, D) EIC of fragment $m / z 617$ from MS/MS on selected ion $m / z$ 851, E) SRM of $m / z 851$ with fragment $m / z 617$, F) EIC of fragment $m / z .563$ from MS/MS on selected ion $m / z, 851$, G) SRM of $m / z, 851$ with fragment $m / z 563$. Corresponding colours behind the chromatograms indicate that the same data set has been used.

\subsubsection{T3Ds and TFsEC in various black teas}

Seven black teas, differing in method of production and/or origin, were analysed for occurrence of T3Ds, TFsECs and theaflavate A (Fig. 3.5). A 'Pu Ehr' tea, which is fermented with exogenous micro-organisms instead of oxidized with endogenous enzymes, was analysed as well. It did not contain any T3Ds, TFsECs or theaflavate A and, therefore, is not further discussed. Based on availability of MS data, as will be addressed later, the method of MS/MS on selected ions was chosen to analyse the tea samples. The areas of the peaks containing the signature fragments were used to semi-quantify the compounds. It should be noted this is a quantification based on MS response, without correction for 
possible differences in ability to ionize between compounds. Therefore, quantities of the same compound can only be compared between different teas, and one should be cautious comparing the quantities of different compounds within the same tea. Ion suppression is another effect that should be considered in MS with complex mixtures, which could cause differences in the quantities analysed. Quantities were expressed in TFg equivalents.

As seen from Figure 3.5, T3Ds and T3Dgs are present in the different teas in various concentrations. Except for the 'Keemun Congou' tea, T3Ds were present in higher concentrations in fully oxidised black teas, in comparison to a semi-oxidized oolong and a smoked tea ('Lapsang Souchong'). This was expected, as T3D is a two-step-oxidation product. Hence, it is tempting to state that the degree of oxidation determines the T3D concentrations. The 'Keemun Congou' tea from the Anhui province of China had low concentrations of T3Ds and T3Dgs. In contrast, the 'Chinese black Yunnan' tea from the province Yunnan of China showed a T3D concentration almost double that of other fully oxidized black teas, and a T3Dg concentration standing out from that of all other teas. This is the first time T3Ds and T3Dgs were found to occur in black teas.

TFsECs were also present in the teas, however, either the MS response was much lower than that for T3Ds or they occurred in much lower concentrations. TFsEC and TFgsEC were present in different teas. Their concentrations did not vary a lot among teas. TFggsEC, reported to be present in black tea, ${ }^{13}$ was not observed.

Theaflavate A was also found in the various teas, in different concentrations. The 'Chinese black Yunnan' showed a particularly high theaflavate A concentration in comparison to the other teas.

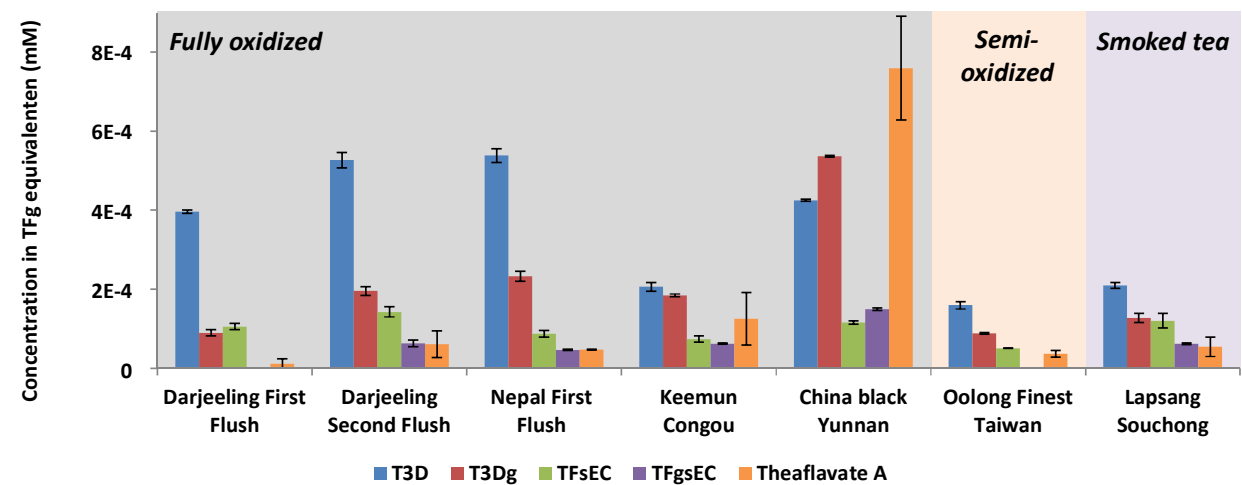

Figure 3.5 Presence, in TFg equivalents, of T3Ds, $\mathrm{TFsEC}^{13}$ and theaflavate $\mathrm{A}$ in various fully oxidized, semi-oxidized or smoked teas. 


\subsection{Discussion}

\subsubsection{MS screening methods for tea phenolics present in low abundance compared}

So far, only EICs from full MS data have been used to screen for oxidized phenolics in tea, ${ }^{15,23}$ except for one recent research where ion mobility measurements showed to be useful in distinguishing isomers from thearubigin fractions of black tea. ${ }^{24}$ The main disadvantage of EICs from full MS data is that no $\mathrm{MS}^{2}$ data of phenolics present in low abundance are recorded, and consequently annotation is often impossible, particularly when analysing complex mixtures. Also, isomers, like T3D, TFsEC and theaflavate A, cannot be distinguished. Both the MS/MS on selected ions method and the SRM method could provide the $\mathrm{MS}^{2}$ data of the compounds. These two methods will be further compared with each other.

The main advantage of the MS/MS on selected ions, compared to SRM, is the way in which the $\mathrm{MS}^{2}$ data are gathered. With MS/MS on selected ions, all $\mathrm{MS}^{2}$ data of the parent ion with the specified $m / z$ value are collected. For SRM, the data gathered is limited to the selected fragment ion belonging to the selected parent ion. This is illustrated by comparing the $\mathrm{MS}^{2}$ fragmentation spectra obtained by both methods in Supplemental Figure S3.3. With SRM, isomers other than the ones screened for are not monitored. For example, theaflavate A, with $m / z 851$ and fragment $\mathrm{m} / \mathrm{z} 579$ visible with the MS/MS on selected ions method (Fig. 3.4C), would not have been found with SRM in the black tea, as it was not specifically screened for. ${ }^{14}$ With MS/MS on selected ions, isomers of $m / z 851$ with $\mathrm{MS}^{2}$ fragments other than $\mathrm{m} / \mathrm{z} 617$ or $\mathrm{m} / \mathrm{z} 563$, including $\mathrm{m} / \mathrm{z} 579$ corresponding to theaflavate A, were also visualized. This provides better insight into the complexity of oxidized tea phenolics.

SRM is preferred when large numbers of samples need quantification, because only the necessary information is monitored and the resolution is always the highest. ${ }^{14,25}$ When analysing black tea to annotate different isomeric two-step-oxidation products, the use of the MS/MS on selected ions method is preferred as it can advance the knowledge on oxidative transformation of tea phenolics. All $\mathrm{MS}^{2}$ data are available for annotation and isomeric products are included.

\subsubsection{Oxidative cascade hypothesis revisited}

By employing the screening method of MS/MS on selected ions in LC-MS runs, the molecular diversity in black tea, described by the 'oxidative cascade hypothesis', was found 
to be even larger than anticipated so far. ${ }^{7}$ Therefore, a refinement of the oligomerization level of the 'oxidative cascade hypothesis' is proposed.

The interflavanic configurations of the one-step-oxidation products, the dimers TF, TS and TC, will be regarded as the basic oligomerization types. Further oligomerization of one of these dimers can occur in two ways: (i) extension via one of the basic oligomerization types, for instance the two-step-oxidation product TFggsEC, which is known to exist in black tea. ${ }^{13}$ Its mono-galloylated and ungalloylated derivatives, TFgsEC and TFsEC, were shown to occur in black tea as well, in the present research. (ii) Extension via a tripartite oligomerization type, an oligomerization in which the preformed basic interflavanic configuration participates by forming a scaffold onto which a third catechin is superimposed, e.g. the two-step-oxidation product T3D. Both T3D and T3Dg were shown to occur in black tea, in the present research. The formation of the two-step-oxidation product TNQ does not involve a second oligomerization step, but an intramolecular rearrangement of the interflavanic configuration of TF into TNQ. Hence, we propose to redefine the oligomerization level of the 'oxidative cascade hypothesis' into three types of oxidation reactions: (i) basic oligomerization, (ii) tripartite oligomerization and (iii) intramolecular rearrangements. Figure 3.6 gives a schematic overview of the different oxidation steps. Combinations of the different oligomerization, rearrangement and hydroxylation reactions, in varying number of oxidation steps, cause the large molecular diversity encountered in black tea thearubigins. ${ }^{7}$

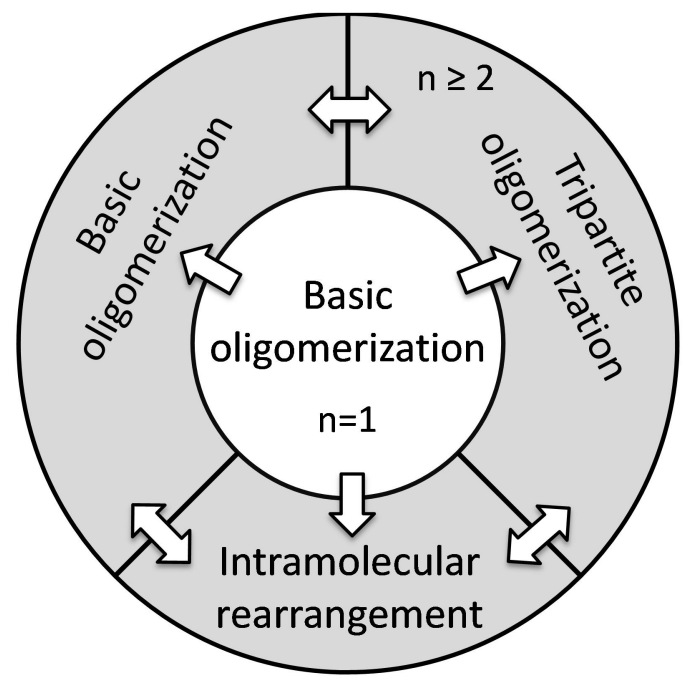

Figure 3.6 Schematic overview of the newly defined oxidation steps in the 'oxidative cascade hypothesis' as proposed in the present research. $\mathrm{n}=$ number of oxidation steps. 


\subsection{References}

1. Chen, Q.; Zhao, J.; Liu, M.; Cai, J. Nondestructive Identification of Tea (Camellia sinensis L.) varieties using FT-NIR spectroscopy and pattern recognition. Czech Journal of Food Sciences 2008, 26, 360-367.

2. Ren, G.; Wang, S.; Ning, J.; Xu, R.; Wang, Y.; Xing, Z.; Wan, X.; Zhang, Z. Quantitative analysis and geographical traceability of black tea using Fourier transform near-infrared spectroscopy (FT-NIRS). Food Research International 2013, 53, 822-826.

3. Versari, A.; Laurie, V. F.; Ricci, A.; Laghi, L.; Parpinello, G. P. Progress in authentication, typification and traceability of grapes and wines by chemometric approaches. Food Research International 2014, 60, 2-18.

4. Osorio, M. T.; Haughey, S. A.; Elliott, C. T.; Koidis, A. Evaluation of methodologies to determine vegetable oil species present in oil mixtures: Proposition of an approach to meet the EU legislation demands for correct vegetable oils labelling. Food Research International 2014, 60, 66-75.

5. López-Gutiérrez, N.; Romero-González, R.; Plaza-Bolaños, P.; Martínez Vidal, J. L.; Garrido Frenich, A. Identification and quantification of phytochemicals in nutraceutical products from green tea by UHPLCOrbitrap-MS. Food Chemistry 2015, 173, 607-618.

6. da Silveira, T. F. F.; Meinhart, A. D.; Ballus, C. A.; Godoy, H. T. The effect of the duration of infusion, temperature, and water volume on the rutin content in the preparation of mate tea beverages: An optimization study. Food Research International 2014, 60, 241-245.

7. Kuhnert, N.; Drynan, J. W.; Obuchowicz, J.; Clifford, M. N.; Witt, M. Mass spectrometric characterization of black tea thearubigins leading to an oxidative cascade hypothesis for thearubigin formation. Rapid Communications in Mass Spectrometry 2010, 24, 3387-3404.

8. Verloop, A. J. W.; Gruppen, H.; Bisschop, R.; Vincken, J. P. Altering the phenolics profile of a green tea leaves extract using exogenous oxidases. Food Chemistry 2016, 196, 1197-1206.

9. Tanaka, T.; Mine, C.; Inoue, K.; Matsuda, M.; Kouno, I. Synthesis of theaflavin from epicatechin and epigallocatechin by plant homogenates and role of epicatechin quinone in the synthesis and degradation of theaflavin. Journal of Agricultural and Food Chemistry 2002, 50, 2142-2148.

10. Opie, S. C.; Clifford, M. N.; Robertson, A. The role of (-)-epicatechin and polyphenol oxidase in the coupled oxidative breakdown of theaflavins. Journal of the Science of Food and Agriculture 1993, 63, 435438.

11. Kusano, R.; Tanaka, T.; Matsuo, Y.; Kouno, I. Structures of epicatechin gallate trimer and tetramer produced by enzymatic oxidation. Chemical and Pharmaceutical Bulletin 2007, 55, 1768-1772.

12. Li, Y.; Shibahara, A.; Matsuo, Y.; Tanaka, T.; Kouno, I. Reaction of the black tea pigment theaflavin during enzymatic oxidation of tea catechins. Journal of Natural Products 2010, 73, 33-39.

13. Yassin, G. H.; Koek, J. H.; Kuhnert, N. Identification of trimeric and tetrameric flavan-3-ol derivatives in the SII black tea thearubigin fraction of black tea using ESI-tandem and MALDI-TOF mass spectrometry. Food Research International 2014, 63, 317-327.

14. Jaffe, J. D.; Keshishian, H.; Chang, B.; Addona, T. A.; Gilette, M. A.; Carr, S. A. Accurate inclusion mass screening: A bridge from unbiased discovery to targeted assay development for biomarker verification. Molecular and Cellular Proteomics 2008, 7, 1952-1962.

15. Kuhnert, N.; Clifford, M. N.; Müller, A. Oxidative cascade reactions yielding polyhydroxy-theaflavins and theacitrins in the formation of black tea thearubigins: Evidence by tandem LC-MS. Food and Function 2010, 1, 180-199.

16. Bohin, M. C.; Vincken, J. P.; Van Der Hijden, H. T. W. M.; Gruppen, H. Efficacy of food proteins as carriers for flavonoids. Journal of Agricultural and Food Chemistry 2012, 60, 4136-4143. 
17. Wu, C.; Xu, H.; Héritier, J.; Andlauer, W. Determination of catechins and flavonol glycosides in Chinese tea varieties. Food Chemistry 2012, 132, 144-149.

18. Tanaka, T.; Betsumiya, Y.; Mine, C.; Kouno, I. Theanaphthoquinone, a novel pigment oxidatively derived from theaflavin during tea-fermentation. Chemical Communications 2000, 1365-1366.

19. Dou, J.; Lee, V. S. Y.; Tzen, J. T. C.; Lee, M. R. Identification and comparison of phenolic compounds in the preparation of oolong tea manufactured by semifermentation and drying processes. Journal of Agricultural and Food Chemistry 2007, 55, 7462-7468.

20. Tanaka, T.; Kouno, I. Oxidation of tea catechins: Chemical structures and reaction mechanism. Food Science and Technology Research 2003, 9, 128-133.

21. Stodt, U. W.; Blauth, N.; Niemann, S.; Stark, J.; Pawar, V.; Jayaraman, S.; Koek, J.; Engelhardt, U. H. Investigation of processes in black tea manufacture through model fermentation (oxidation) experiments. Journal of Agricultural and Food Chemistry 2014, 62, 7854-7861.

22. Wan, X.; Nursten, H. E.; Cai, Y.; Davis, A. L.; Wilkins, J. P. G.; Davies, A. P. A new type of tea pigment from the chemical oxidation of epicatechin gallate and isolated from tea. Journal of the Science of Food and Agriculture 1997, 74, 401-408.

23. Yassin, G. H.; Koek, J. H.; Jayaraman, S.; Kuhnert, N. Identification of novel homologous series of polyhydroxylated theasinensins and theanaphthoquinones in the SII fraction of black tea thearubigins using ESI/HPLC tandem mass spectrometry. Journal of Agricultural and Food Chemistry 2014, 62, 9848-9859.

24. Yassin, G. H.; Grun, C.; Koek, J. H.; Assaf, K. I.; Kuhnert, N. Investigation of isomeric flavanol structures in black tea thearubigins using ultraperformance liquid chromatography coupled to hybrid quadrupole/ion mobility/time of flight mass spectrometry Journal of Mass Spectrometry 2014, 49, 10861095.

25. Yocum, A. K.; Chinnaiyan, A. M. Current affairs in quantitative targeted proteomics: Multiple reaction monitoring - mass spectrometry. Briefings in Functional Genomics and Proteomics 2009, 8, 145-157.

\subsection{Supplementary data}

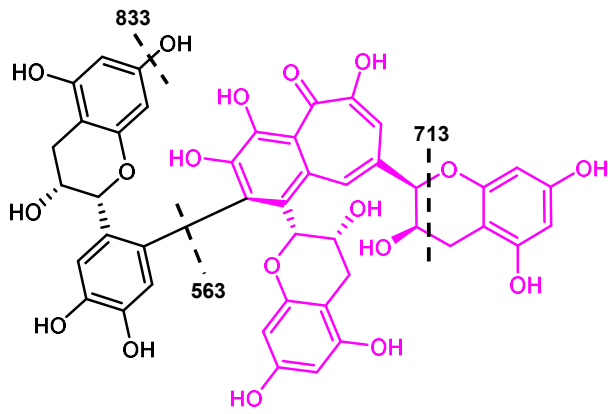

Figure S3.1 Structural depiction of the main fragmentation routes of TFsEC.13 The signature fragment of $m / z 563$ is shown in pink. 


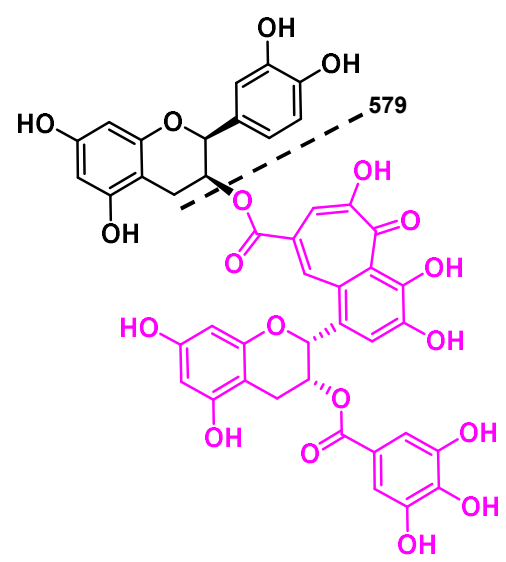

Figure S3.2 Structural depiction of the main fragmentation route of theaflavate A.21,22 The signature fragment of $m / z, 579$ is shown in pink.
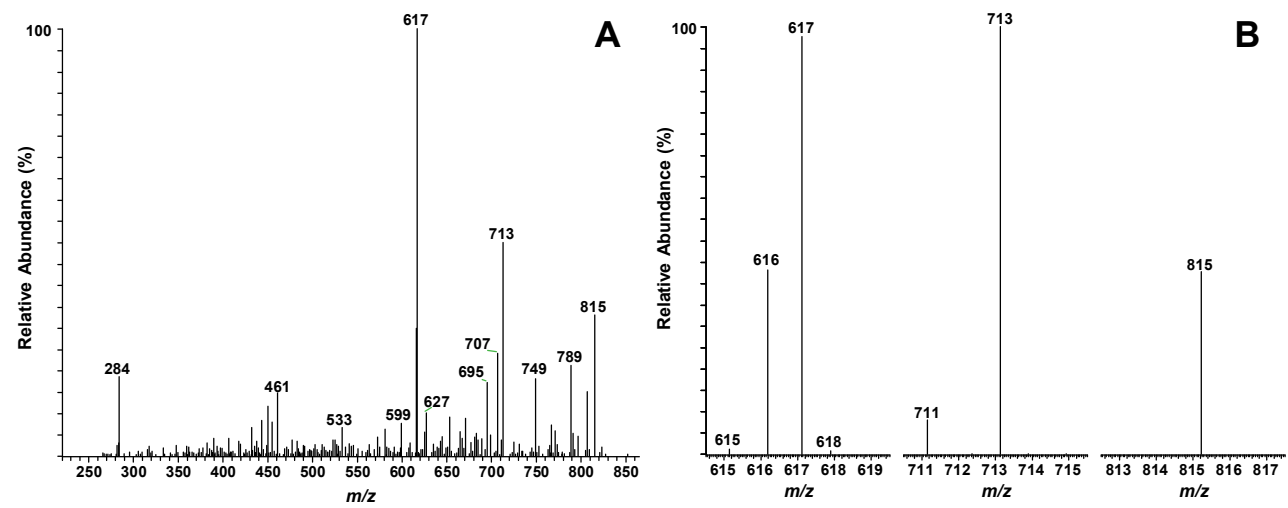

Figure S3.3 MS spectra from a Chinese black Yunnan tea. A) MS spectrum from an MS/MS measurement on the selected ion of $m / z 851$, B) MS spectrum from an SRM measurement of the fragments $m / z 617, m / z 713$ and $m / z 815$ of parent ion $m / z 851$. 
Chapter 3 


\title{
Chapter 4
}

\section{Structural characterization of different dehydrocatechin oligomers by MS/MS, and their occurrence in black tea}

\begin{abstract}
Dehydrocatechins $(\mathrm{DhC})$, oligomeric oxidation products of (epi)catechins, were formed in model incubations of epicatechin with mushroom tyrosinase. DhC oligomers up to tetramers were detected by RP-UHPLC-MS analysis. Measurements with MALDI-TOFMS showed formation of oligomers up to at least 15 catechin subunits. Isomeric DhCs were obtained, and a method based on $\mathrm{MS}^{2}$ fragment ratios was set up to distinguish between the different interflavanic configurations of the isomers. In the model incubation, 8 dehydrodicatechins $\left(\mathrm{DhC}_{2}\right)$ and 22 dehydrotricatechins $\left(\mathrm{DhC}_{3}\right)$ were tentatively annotated by their $\mathrm{MS}^{2}$ signature fragments. Three different interflavanic configuration-types were annotated for the $\mathrm{DhC}_{2} \mathrm{~s}$. $\mathrm{DhC}_{2} \mathrm{~s}$ and $\mathrm{DhC}_{3} \mathrm{~s}$ were shown to occur in a black tea extract for the first time. For the $\mathrm{DhC}_{2}$ s, at least two isomeric types, i.e. $\mathrm{DhC} \beta$ and $\mathrm{DhC} \varepsilon$, could be annotated in black tea.
\end{abstract}




\subsection{Introduction}

When green tea leaves are processed into black tea, the green leaf phenolics are oxidized by the leaves' endogenous enzymes. The main phenolic compounds in green tea leaves are catechins (also referred to as flavan-3-ols), characterized by their meta-5,7dihydroxy substituted A-ring and di- or trihydroxylic B-ring. These catechins form the set of starting compounds with limited molecular diversity that after oxidation results in the estimated 10,000 different complex phenolics in black tea, the thearubigins. ${ }^{l}$ The formation of this array of phenolics is explained by the 'oxidative cascade hypothesis' (Ch. 3). ${ }^{l}$

The 'oxidative cascade' consists of three types of oxidation reactions: (i) oligomerization via either a basic-type or a tripartite-type, (ii) intramolecular rearrangement, and (iii) hydroxylation. Oligomerization of catechins can result in different interflavanic configurations. An interflavanic configuration refers to the cross-link between two subunits, also including the structural changes occurring within a molecule as a result of the coupling reaction. The basic oligomerization-types are the interflavanic configurations that result from one oxidation step: the theaflavin (TF) subtype, the theasinensin (TS) subtype, and the theacitrin (TC) subtype, the characteristics of which are a benzotropolone moiety, a C-C bond, and two fused pentameric rings, respectively. The intramolecular rearrangements and tripartite-oligomerizations always require a second oxidation step, in which one of the preformed basic interflavanic configurations participates, by rearrangement (no elongation; dimeric product, e.g. $\mathrm{TNQ}^{2}$ and theacoumarin ${ }^{3}$ ), or by forming a scaffold onto which a third catechin is superimposed (branching; trimeric product, e.g. T3D $(\mathbf{C h . ~ 3})^{4}$ ), respectively. Furthermore, extra oxidation steps can also elongate oligomers, simply by attaching subunits through one of the basic oligomerization types $(\mathbf{C h} . \mathbf{3})$. The numerous different combinations of oxidation reactions are thought to cause the extensive molecular diversity of thearubigins present in black tea. ${ }^{l}$ Oligomers up to a degree of polymerization (DP) of seven catechin subunits are expected in black tea, based on a maximum molecular mass $(\mathrm{Mw})$ of 2,100 Da reported for black tea phenolics. ${ }^{l}$

The increase in molecular diversity when converting green tea leaves into black tea is an uncontrolled process. Only control of temperature ${ }^{5}$ and $\mathrm{pH}^{6}$ during fermentation, with respect to manufacturing high quality black tea, has been investigated, so far. An opportunity for a more controlled oxidation process to generate black tea would be to physically separate the phenolics and oxidative enzymes, e.g. polyphenol oxidase and peroxidase, from tea leaves in their native states, prior to the oxidation process. Instead of cutting and crushing the leaves to bring the catechins and oxidative enzymes in contact, the catechins and enzymes can be added together in the desired amounts and conditions. Previous research, using model systems starting from epicatechin (EC) and epigallocatechin 
(EGC), indicated that tyrosinase yielded more defined end products of EC with EGC, i.e. theaflavin (TF) and theatridimensins (T3D), than laccase. The latter further oxidized the TFs into insoluble compounds. ${ }^{7}$ Using these model systems, novel oxidation products were first discovered in model incubations, ${ }^{4}$ after which they were identified in black tea, for example T3Ds (Ch. 3).

In the present study, another potential starting point for the formation of complex phenolics in black tea is investigated, i.e. the oligomerization products from $\mathrm{EC}$ subunits alone. Dehydrodicatechins $\left(\mathrm{DhC}_{2}\right)$ (Fig. 4.1), A-ring to B-ring linked catechin dimers, were shown to be formed when incubated with grape polyphenol oxidase. ${ }^{8}$ In previous research using model incubations of catechins with tyrosinase, the formation of $\mathrm{DhC}_{2} \mathrm{~s}$ and dehydrotricatechins $\left(\mathrm{DhC}_{3}\right)$ was observed as well. ${ }^{7}$

A

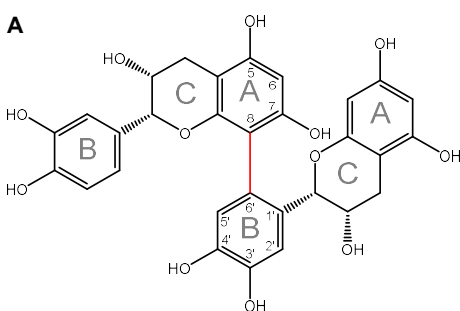

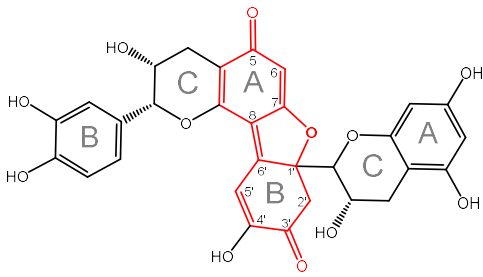

B

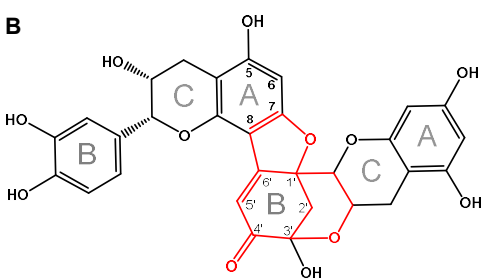

D

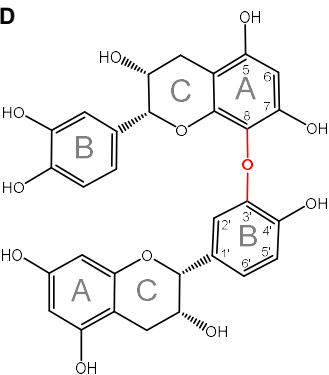

Figure 4.1 Structural formulas of different dehydrodicatechin dimers: (A) EC- $\beta_{\mathrm{AB}}-\mathrm{EC}$ with $\mathrm{m} / \mathrm{z}$ 577. (B) EC- $\gamma_{\mathrm{AB}}-\mathrm{EC}$ with $m / z$ 575. (C) EC- $\delta_{\mathrm{AB}}-\mathrm{EC}$ with $m / z$ 575. (D) EC- $\varepsilon_{\mathrm{AB}}-\mathrm{EC}$ with $m / z .577 .8$ The different interflavanic configurations are indicated in red. The nomenclature proposed for dehydrocatechins is elaborated in Figure 4.3.

Figure 4.1 shows four different interflavanic configurations for DhCs. (i) A single C-C bond (Fig. 4.1A), (ii) an ether bond and a single $\mathrm{C}-\mathrm{C}$ bond in between the catechin subunits, plus an additional intraflavanic ether linkage from the C-ring to the B-ring and a carbonyl group for one of the catechin subunits, resulting after intramolecular rearrangement (Fig. 4.1B), (iii) an ether bond and a double $\mathrm{C}-\mathrm{C}$ bond, plus a carbonyl group for both of the subunits, resulting after intramolecular rearrangement (Fig. 4.1C), and (iv) a single ether bond (Fig. 4.1D). The structures shown in Figure 4.1 represent the 
interflavanic configurations of the A- and B-rings that are energetically most favourable. Isomers can occur as well, involving different positions on the A and B-rings for crosslinking, via one of these four DhC interflavanic configuration types. ${ }^{8}$

DhCs oligomerize via B-ring oxidation of $(\mathrm{E}) \mathrm{C}$ subunits. ${ }^{8}$ This B-ring oxidation is predominant in tea oxidation reactions as well..$^{9}$ Therefore, it is hypothesized that DhCs will also be formed during the oxidation process that results in black tea, but their presence in tea has not been established yet, to the best of our knowledge. Oligomers of A-ring to Cring linked monomeric catechins, the procyanidins (PC), are isomers of DhCs, and are known to occur in green tea leaves. ${ }^{9-11}$

The aim of the present research is to specifically analyse various oxidized teas for the presence of DhCs with different interflavanic configurations. To analyse the various DhCs in a complex mixture of phenolics, like black tea, a selective mass spectrometric screening method is used (Ch. 3). In order to use this method, the fragmentation patterns of the different DhCs need to be established, to be able to define their signature fragments. These signature fragments will be used to distinguish DhCs from PCs, and to distinguish the different interflavanic configurations in DhCs. A model system of EC with mushroom tyrosinase will be used to prepare $\mathrm{DhCs}$, to obtain those signature fragments.

\subsection{Materials and Methods}

\subsubsection{Materials}

(-)-Epicatechin (EC), theaflavin-3-gallate $(\mathrm{TFg})$ and mushroom tyrosinase were purchased from Sigma Aldrich (St. Louis, MO, USA). Anhydrous citric acid was purchased from Acros Organics (Geel, Belgium) and sodium dihydrogen phosphate and disodium hydrogen phosphate from Merck (Darmstadt, Germany). A commercially available procyanidin extract from grape seeds $\left(\right.$ Vitaflavan $\left.^{\circledR}\right)$ was kindly provided by Levita Chemicals International NV (Antwerpen, Belgium). Various teas from different origin were obtained from a local store: Darjeeling First Flush (India), Darjeeling Second Flush (India), Nepal First Flush (Nepal), Keemun Congou (Anhui, China), China black Yunnan (Yunnan, China), Oolong Finest Taiwan (Taiwan), Pu Ehr (China), and Lapsang Souchong (China). 2,5-Dihydroxybenzoic acid (DHB) was purchased from Bruker Daltronics (Bremen, Germany). Maltodextrin MD20 (DP 1 to 20) was obtained from AVEBE (Veendam, The Netherlands). Ultra-high-performance liquid chromatography mass spectrometry (UHPLCMS) grade acetonitrile $(\mathrm{ACN})$ and methanol $(\mathrm{MeOH})$ were obtained from Biosolve BV (Valkenswaard, The Netherlands). Water was prepared using a Milli-Q water purification system (Millipore, Billerica, MA, USA). 


\subsubsection{Incubation of epicatechin with tyrosinase}

EC $(1 \mathrm{mM})$ was incubated with tyrosinase at $0.0001 \mathrm{U} / \mathrm{mL}$ (one unit equalling the amount of enzyme that consumes $1 \mu \mathrm{mol}$ of oxygen per min for oxidation of catechin at $\mathrm{pH}$ 5.5 and $25{ }^{\circ} \mathrm{C}$ ). Incubations were performed in citric acid-sodium phosphate buffer (30 $\mathrm{mM}) \mathrm{pH} 5.5$ or sodium phosphate buffer $(20 \mathrm{mM}) \mathrm{pH}$ 8.0. Samples $(1 \mathrm{~mL})$ were incubated at $25^{\circ} \mathrm{C}$ for $5 \mathrm{~min}$. The samples were centrifuged $\left(10,000 \mathrm{~g}, 5 \mathrm{~min}, 20^{\circ} \mathrm{C}\right)$ and analysed by reversed-phase ultra-high-performance liquid chromatography mass spectrometry (RPUHPLC-MS) and matrix-assisted laser desorption/ionization time-of-flight mass spectrometry (MALDI-TOF-MS). Compounds were tentatively annotated by mass spectrometry.

\subsubsection{Extraction of black tea phenolics}

Black tea $(0.5 \mathrm{~g})$ was three times extracted with $50 \mathrm{~mL}$ boiling water for $10 \mathrm{~min}$ under continuous stirring. Extracts were filtered through filter paper (Grade 1, Whatman, Chalfont St. Giles, UK) by Büchner filtration and the filtrates were combined. The samples were centrifuged $\left(10,000 \mathrm{~g}, 5 \mathrm{~min}, 20^{\circ} \mathrm{C}\right)$ prior to analysis by RP-UHPLC-MS.

\subsubsection{RP-UHPLC}

Samples were analysed on an Accela UHPLC system (Thermo Scientific, San Jose, CA, USA) equipped with a pump, an autosampler at $15^{\circ} \mathrm{C}$, and a photodiode array (PDA) detector. Samples $(1 \mu \mathrm{L})$ were injected onto a Hypersil Gold column $(2.1 \times 150 \mathrm{~mm}$, particle size of $1.9 \mu \mathrm{m}$, Thermo Scientific). Water acidified to $0.1 \%(\mathrm{v} / \mathrm{v})$ acetic acid (eluent A) and ACN acidified to $0.1 \%(\mathrm{v} / \mathrm{v})$ acetic acid (eluent B) were used as eluents. The flow rate was $300 \mu \mathrm{L} / \mathrm{min}$ and the column oven temperature was controlled at $30^{\circ} \mathrm{C}$. The PDA detector was set to measure $200-600 \mathrm{~nm}$. The following elution profile was used: $0-1 \mathrm{~min}$, isocratic on $5 \%(\mathrm{v} / \mathrm{v}) \mathrm{B} ; 1-12 \mathrm{~min}$, linear gradient from 5-55\% (v/v) B; 12-13 min, linear gradient from $55-100 \%(\mathrm{v} / \mathrm{v}) \mathrm{B} ; 13-18 \mathrm{~min}$, isocratic on $100 \%(\mathrm{v} / \mathrm{v}) \mathrm{B} ; 18-18.1 \mathrm{~min}$, linear gradient from 100-5\% (v/v) B; and 18.1-22 min, isocratic on 5\% (v/v) B.

\subsubsection{Electrospray Ionization - Mass Spectrometry (ESI-MS)}

Mass spectrometric data were obtained on a Velos Pro mass spectrometer (Thermo Scientific) equipped with an ESI probe coupled to the RP-UHPLC system. Nitrogen was used as sheath gas and auxiliary gas. Data were collected over different $\mathrm{m} / \mathrm{z}$ ranges, specific for the compounds of interest. The $\mathrm{m} / z$ ranges were $m / z$ 565-582 for EC dimers, $m / z$ 855870 for EC trimers and $m / z 1140-1160$ for EC tetramers. Data-dependent $\mathrm{MS}^{\mathrm{n}}$ analysis was performed with a normalized collision energy of $35 \%$. The $\mathrm{MS}^{\mathrm{n}}$ fragmentation was performed on the most intense product ion in the $\mathrm{MS}^{\mathrm{n}-1}$ spectrum. The system was tuned with TFg in negative ionization (NI) mode. Most settings were optimized via automatic 
tuning using "Tune Plus" (Xcalibur 2.07, Thermo Scientific). The transfer tube temperature was $350{ }^{\circ} \mathrm{C}$, and the source voltage was $4.0 \mathrm{kV}$. Data acquisition and reprocessing were performed with Xcalibur 2.07 (Thermo Scientific). The MS was also run with MS/MS on selected ions settings. With MS/MS on selected ions, the MS only monitors the $\mathrm{MS}^{2}$ data of the parent ions specified in the MS/MS inclusion list, during a specified timeframe.

Compounds were quantified by MS signal relative to TFg, and the amounts were expressed as TFg equivalents. The TFg calibration curve (ranging from 0.0006-0.004 mM) had a correlation coefficient of 0.99 .

\subsubsection{MALDI-TOF-MS}

Samples were prepared as described, without including a desalting step. A solution of $10 \mathrm{mg} / \mathrm{mL}$ of DHB in water was used as matrix. Spots were prepared on an MTP 384 ground steel target plate (Bruker Daltonics): $0.5 \mu \mathrm{L}$ of DHB solution was applied and dried under a stream of air, then $0.5 \mu \mathrm{L}$ of sample was applied on top of it and dried under a stream of air, finally $0.5 \mu \mathrm{L}$ of DHB solution was applied on top of it and dried under a stream of air. Samples were analysed on an Ultraflextreme workstation equipped with a Smartbeam II laser of $355 \mathrm{~nm}$, operated in positive mode and controlled by FlexControl 3.4 software (Bruker Daltonics). Spectra were collected at a laser intensity between $55 \%$ and $65 \%$ in reflector mode with voltages of ion sources 1 and 2 , reflectors 1 and 2 and the lens set at $25.01 \mathrm{kV}, 22.37 \mathrm{kV}, 26.45 \mathrm{kV}, 13.32 \mathrm{kV}$ and $8.34 \mathrm{kV}$, respectively. Laser frequency was $1000 \mathrm{~Hz}$. Calibration was performed with maltodextrin and a mass scan range from 200-3500 Da was used with matrix suppression up to $250 \mathrm{Da}$. Data analysis was conducted using FlexAnalysis v3.3 software (Bruker Daltonics).

\subsection{Results and Discussion}

\subsubsection{Degree of polymerization of dehydrocatechins}

It was hypothesized, based on previous results, ${ }^{7}$ that tyrosinase would form DhCs from EC. During the incubation of EC with tyrosinase precipitation occurred. Both the supernatant and the precipitate, which was solubilized in $\mathrm{MeOH}$, were analysed by MALDI-TOF-MS. Figure 4.2 shows the spectrum of the solubilised precipitate. A clear pattern of peaks was visible, with intervals between peaks of $288 \mathrm{Da}$, corresponding to the $\mathrm{Mw}$ of an EC moiety. DhCs with DPs up to at least 15 EC subunits were formed. In the supernatant the same clear peak pattern was found, showing DhCs with DPs up to 11 (data not shown). These numbers exceeded the maximum DP of 7 found for oxidized phenolics in black tea. ${ }^{1,12}$ 


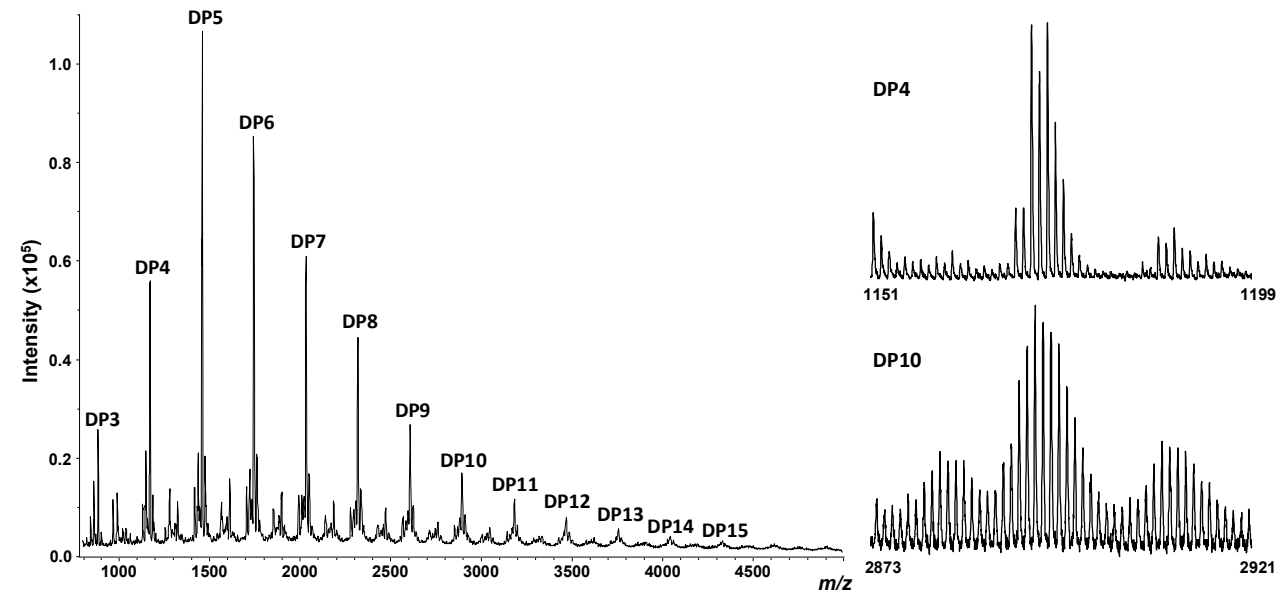

Figure 4.2 MALDI-TOF-MS spectrum of the precipitate of a one hour EC incubation using tyrosinase, with a zoom of the DP 4 and DP 10 peak.

When zooming in on the peaks representing the various DPs in the spectrum (Fig. 4.2), it was clear that these peaks actually consisted of multiple signals, with the individual signals differing by $1 \mathrm{Da}$ from each other. When comparing the DP 4 and DP 10 peaks in Figure 4.2, it seemed that the DP 4 peak contained around eight individual signals, whereas the DP 10 peak contained at least 14 signals. This peak broadening with increasing DP was explained by the increased number of interflavanic configurations of DhCs. The dimers in Figures 4.1B and 4.1C have two bonds between the subunits. These dimers were formed with a concomitant loss of $4 \mathrm{Da}$ upon coupling of the two molecules, whereas the dimers in Figures 4.1A and 4.1D have a single bond interflavanic linkage with a concomitant loss of only $2 \mathrm{Da}$ upon coupling. These different interflavanic configurations explain the $2 \mathrm{Da}$ difference in $\mathrm{Mw}$ observed for the signals within one peak. The presence of ${ }^{13} \mathrm{C}$ isotopes, contributing $1 \mathrm{Da}$ extra to the $\mathrm{Mw}$, adds further to the complexity of the spectrum, as an individual compound can be represented by multiple signals. The higher the DP, the more different interflavanic configurations can occur, along with an increased probability of incorporating one or more ${ }^{13} \mathrm{C}$ isotopes in the oligomer, resulting in wider peaks for each DP in the spectra, but with lower individual intensities.

The decrease in intensity for the higher DP peaks did not necessarily reflect lesser amounts. The ionization of larger molecules is known to be less good than that of smaller ones. ${ }^{13-15}$ MALDI-TOF-MS had an advantage over UHPLC-MS in detecting the larger DPs, because all isomers contributed to the same signal, which facilitated their collective detection. To annotate the different compounds by their $\mathrm{MS}^{2}$ fragments, MALDI-TOF-MS could not be used, because it had a fragmentation frame of $5 \mathrm{Da}$ in which it fragments all 
compounds. To analyse the different isomeric DhCs and to annotate them by their $\mathrm{MS}^{2}$ fragmentation pattern, RP-UHPLC-MS measurements were performed.

\subsubsection{Nomenclature of dehydrocatechins}

The number of possible DhCs formed increased with DP, and the current nomenclature would not cover all the possible molecules satisfactory. DhCs are named similarily to procyanidins (PCs), on the basis of an alphanumeric system. ${ }^{16-18}$ The DhCs A are catechin dimers linked via two interflavanic bonds (Fig. 4.1 B,C), ${ }^{8,17}$ whereas the DhCs B are catechin dimers linked via a single interflavanic bond (Fig. 4.1 A,D) ${ }^{8,18}$ Clearly, this alphanumeric system does not consider the different types of interflavanic configurations within these DhC A and DhC B isomers. Also, it is not easily extended towards oligomers. Therefore, we propose a new nomenclature, based on a procyanidin nomenclature, but different from the one mentioned above. ${ }^{19}$

This new nomenclature addresses the different types of interflavanic configurations between subunits with a Greek letter. The catechin subunits linked will be mentioned (in abbreviated form) in front and after the Greek letter, starting from the terminal unit. The terminal unit of DhCs is defined as the catechin moiety with the unreacted B-ring (Fig. 4.3A). All other subunits are extension units. In PCs the terminal unit is defined as the subunit with the unreacted C-ring (Fig. 4.3A).

Figure 4.3A schematically represents the five types of interflavanic configurations known to occur in DhCs and PCs, distinguished by the Greek letters, $\alpha, \beta, \gamma, \delta$, and $\varepsilon$. The single $\mathrm{C}-\mathrm{C}$ bond between the $\mathrm{DhC}$ subunits is comparable to the single $\mathrm{C}-\mathrm{C}$ bond between subunits of B-type PCs, also referred to as $\beta$-configuration ${ }^{19}$. The difference between DhCs and PCs relates to the rings involved in connecting the two subunits, being $A \rightarrow B$ and $\mathrm{A} \rightarrow \mathrm{C}$, respectively. Therefore, from now on, the two interlinked rings, starting from the terminal side, are added as a subscript to the Greek letter representing the interflavanic configuration. The first $\mathrm{DhC}$ dimer in Figure 4.3A is referred to as $\mathrm{EC}-\beta_{\mathrm{AB}}-\mathrm{EC}$, whereas its isomer, B-type $\mathrm{PC}$, is referred to as $\mathrm{EC}-\beta_{\mathrm{AC}}-\mathrm{EC}$. 
A

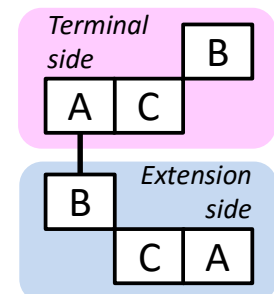

$E C-\beta_{A B}-E C$

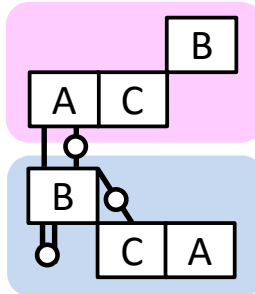

$E C-\gamma_{A B}-E C$

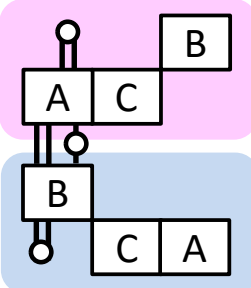

$E C-\delta_{A B}-E C$

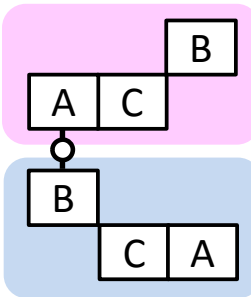

$E C-\varepsilon_{A B}-E C$

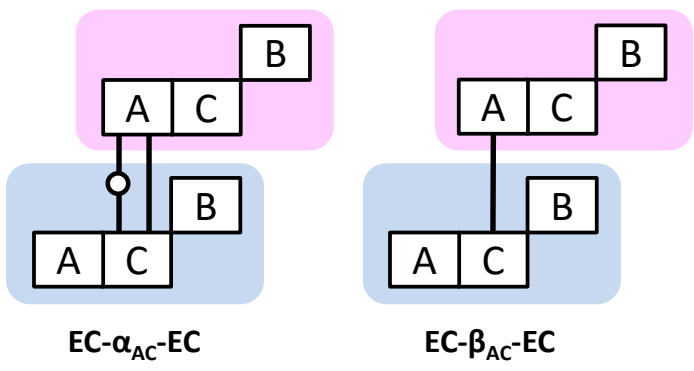

B

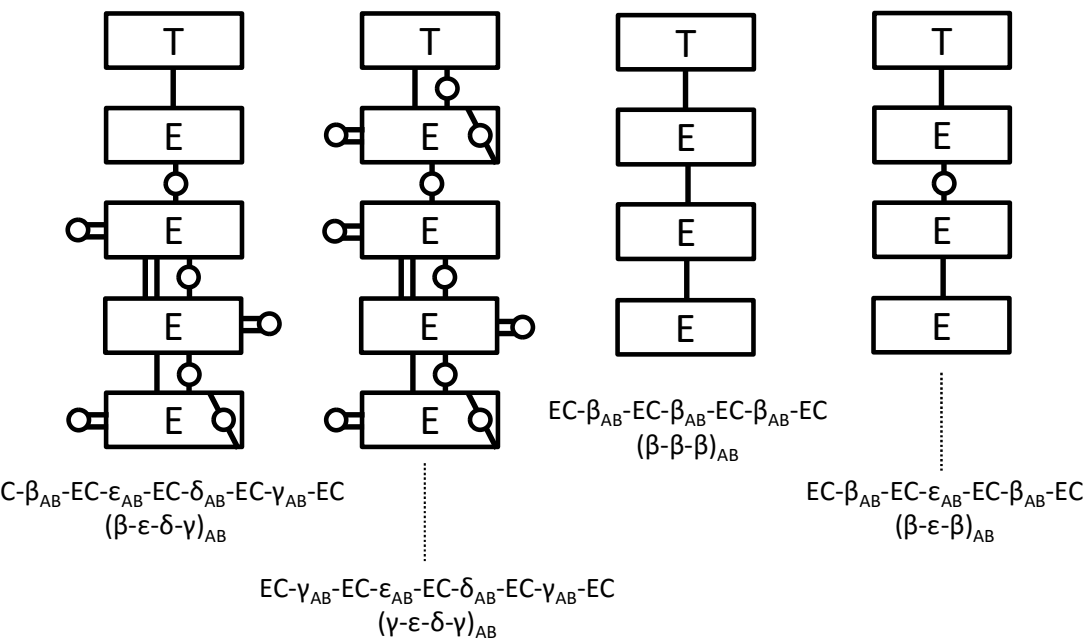

Figure 4.3 Schematic representation of dehydrocatechins and procyanidins. (A) The different types of interflavanic A-ring to B-ring and A-ring to C-ring configurations. Pink shade: terminal side of the dimer, blue shade: extension side of the dimer. (B) Examples of the proposed nomenclature for dehydrocatechins. T: terminal subunit of the oligomer; E: extension subunits of the oligomer.

The two bonds between the subunits of an A-type PC are an ether bond and a C-C bond, referred to as an $\alpha$-configuration (Fig 4.3A). ${ }^{19} \mathrm{In}$ the $\mathrm{DhC}$ interflavanic configuration types, the $\alpha$-type does, to the best of our knowledge, only occur as an unstable intermediate 
product after oxidation of $E C-\beta_{\mathrm{AB}}-\mathrm{EC}{ }^{8}{ }^{8}$ Subsequently, $\mathrm{EC}-\alpha_{\mathrm{AB}}-\mathrm{EC}$ quickly rearranges into an interflavanic configuration consisting of an ether bond, a $\mathrm{C}-\mathrm{C}$ bond and additionally an intraflavanic ether bond from the $\mathrm{C}$-ring to the $\mathrm{B}$-ring and a carbonyl group in the extension subunit. This interflavanic configuration is, from now on, referred to as a $\gamma$-configuration, $\mathrm{EC}-\gamma_{\mathrm{AB}}-\mathrm{EC}$ (Fig. 4.3A). Alternatively, $\mathrm{EC}-\alpha_{\mathrm{AB}}-\mathrm{EC}$ can rearrange into an interflavanic configuration with a double bond and an ether bond between the catechin subunits, and a carbonyl group on both subunits. This interflavanic configuration is, from now on, referred to as $\delta$-configuration, $\mathrm{EC}-\delta_{\mathrm{AB}}-\mathrm{EC}$ (Fig. 4.3A). The last interflavanic configuration potentially present in $\mathrm{DhCs}$ is an ether bond between the catechin subunits, which is, from now on, referred to as $\varepsilon$-configuration, $\mathrm{EC}-\varepsilon_{\mathrm{AB}}-\mathrm{EC}$ (Fig. 4.3A).

The positions of the cross-links in the interflavanic configuration can differ, leading to various isomers. For instance, $\mathrm{EC}-\varepsilon_{\mathrm{AB}}-\mathrm{EC}$ in Figure 4.1D is 8-3' linked, whereas it could also be 8-4' linked, ${ }^{8}$ 6-3' linked, or 6-4' linked. The nomenclature proposed could potentially be extended by defining the positions of the cross-links. As positional isomers could not be annotated with MS/MS, the nomenclature was not further refined in this study, for sake of clarity.

Figure 4.3B schematically shows examples of this nomenclature for a number of theoretical DhC pentamers and tetramers. As the nomenclature can become quite extensive, an abbreviated nomenclature is given as well, in which the subunits are left out. In case of $\mathrm{DhCs}$ and PCs, the subunits of the oligomers are always (E)Cs. The subunits might have been omitted from the nomenclature altogether. However, it was decided to keep the subunits in the nomenclature, to be able to extend the nomenclature further to other (oxidized) tea phenolics, as TF, TS, TC, etc...

\subsubsection{Number of dehydrocatechin isomers formed by tyrosinase}

RP-UHPLC-MS measurements with narrow mass detection ranges $(\Delta m / z \quad 15-20)$ were performed in order to visualize DhCs by LC-MS. Figure 4.4 shows the MS extracted ion chromatograms (EIC) for the different narrow mass ranges of the incubation of EC with tyrosinase. The characteristics of the peaks are shown in Table 4.1.

Eight $\mathrm{DhC}_{2} \mathrm{~S}$ were distinguished (Fig. 4.4A), with $\mathrm{m} / \mathrm{z}$ values of 575 (8) or 577 (1-7). The number of peaks increases with degree of polymerization (DP), because the probability of incorporating more interflavanic configurations increases, as does the number of possibilities in their order. This can be seen in Figures 4.4B and 4.4C, where the number of peaks increased from $\mathrm{DhC}_{3} \mathrm{~s}$ to $\mathrm{DhC}_{4} \mathrm{~s}$, respectively. From $\mathrm{DhC}_{5} \mathrm{~s}$ onwards, the isomer signals seemed too scattered over the chromatogram, and they could not be individually detected anymore (data not shown). Thus, RP-UHPLC-MS measurements can be used to detect $\mathrm{DhCs}$ up to $\mathrm{DhC}_{4} \mathrm{~s}$. 


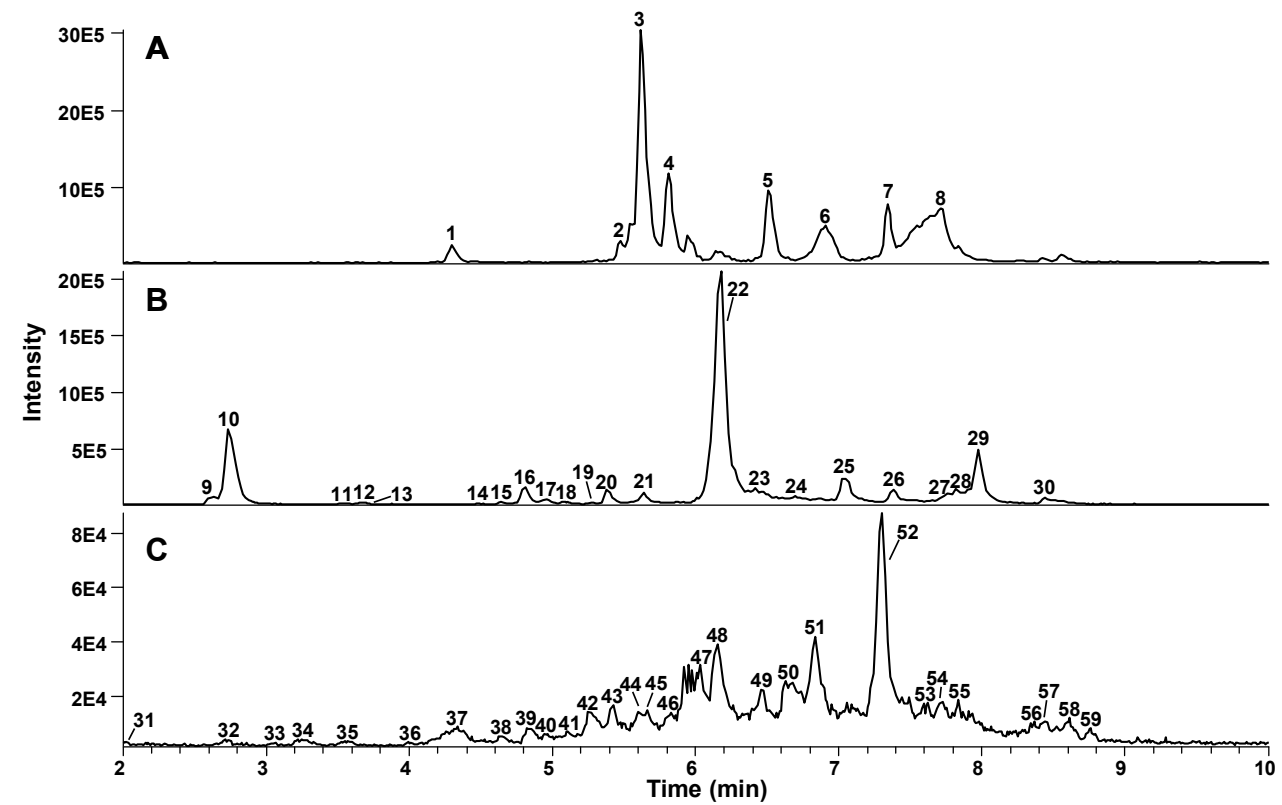

Figure 4.4 MS base peak extracted ion chromatograms of EC incubated with tyrosinase. Measured MS range is (A) $m / z 565-582$ for dimers, extracted ions: $m / z 575,577$, (B) $m / z$ 855-870 for trimers, extracted ions: $m / z 861,863,865$ and $(\mathbf{C}) \mathrm{m} / \mathrm{z}_{1} 1140-1160$ for tetramers, extracted ions: $\mathrm{m} / \mathrm{z} 1147$, $1149,1151,1153$. The compounds corresponding to the numbered peaks are listed in Table 4.1 .

Table 4.1 Compounds found by RP-UHPLC-MS $\mathrm{n}$ after incubation of EC with tyrosinase.

\begin{tabular}{|c|c|c|c|c|c|}
\hline $\begin{array}{l}\text { Peak } \\
\text { no. }\end{array}$ & $\begin{array}{l}\text { Retention } \\
\text { time } \\
\text { (min) }\end{array}$ & $\begin{array}{c}{[\mathrm{M}-\mathrm{H}]^{-}} \\
\mathrm{m} / \mathrm{z}\end{array}$ & $\begin{array}{l}\text { MS }^{2} \text { fragments } \\
m / z \text { (intensity) }\end{array}$ & $\mathrm{MS}^{3}$ fragments $m / z$ & $\begin{array}{r}\text { Tentative } \\
\text { annotation }\end{array}$ \\
\hline \multicolumn{6}{|c|}{ Dehydrodicatechins } \\
\hline \multicolumn{6}{|c|}{$m / z 575$ (theoretical number of isomers: 18 ) } \\
\hline 8 & 7.71 & 575 & $\begin{array}{l}\text { 449(100), 287(32), 437(31), } \\
407(17), 394(14)\end{array}$ & $\begin{array}{l}449: 287,405,269 \\
243,394\end{array}$ & $\delta_{\mathrm{AB}}$ \\
\hline \multicolumn{6}{|c|}{$\mathrm{m} / \mathrm{z} 577$ (theoretical number of isomers: 10 ) } \\
\hline \multirow[t]{2}{*}{1} & 4.30 & 577 & $\begin{array}{l}\text { 439(100), 393(92), 533(89), } \\
541(57), 515(52), 421(38) \text {, } \\
329(31)\end{array}$ & $\begin{array}{l}\text { 439: } 395,377,353, \\
297,247\end{array}$ & $\beta_{\mathrm{AB}}$ \\
\hline & & & & $515: 435,457,475$ & \\
\hline \multirow[t]{2}{*}{2} & 5.48 & 577 & $\begin{array}{l}\text { 393(100), 533(96), 439(92), } \\
425(90), 515(59), 541(58), \\
421(40), 329(39)\end{array}$ & $\begin{array}{l}\text { 393: } 269,283,271, \\
229,241\end{array}$ & $\beta_{\mathrm{AB}}$ \\
\hline & & & & $533: 449,489,353$ & \\
\hline
\end{tabular}




\begin{tabular}{|c|c|c|c|c|c|}
\hline $\begin{array}{l}\text { Peak } \\
\text { no. }\end{array}$ & $\begin{array}{l}\text { Retention } \\
\text { time (min) }\end{array}$ & $\begin{array}{c}{[\mathrm{M}-\mathrm{H}]^{-}} \\
m / z\end{array}$ & $\begin{array}{c}\mathrm{MS}^{2} \text { fragments } \\
m / z \text { (intensity) }\end{array}$ & $\mathrm{MS}^{3}$ fragments $m / z$ & $\begin{array}{l}\text { Tentative } \\
\text { annotation" }\end{array}$ \\
\hline \multirow[t]{2}{*}{3} & 5.62 & 577 & $\begin{array}{l}\text { 439(100), 425(74), 393(69), } \\
533(42), 269(33)\end{array}$ & $\begin{array}{l}439: 377,247,289 \\
395\end{array}$ & $\beta_{\mathrm{AB}}$ \\
\hline & & & & $\begin{array}{l}425: 241,287,262 \\
381,389\end{array}$ & \\
\hline 4 & 5.81 & 577 & $\begin{array}{l}\text { 439(100), 425(97), 393(95), } \\
533(54), 269(41)\end{array}$ & $\begin{array}{l}\text { 439: } 377,247,289 \\
395\end{array}$ & $\beta_{\mathrm{AB}}$ \\
\hline 5 & 6.51 & 577 & $\begin{array}{l}425(100), 439(44), 533(43), \\
287(43), 289(38)\end{array}$ & $\begin{array}{l}425: 287,299,363 \\
381,241\end{array}$ & $\varepsilon_{\mathrm{AB}}$ \\
\hline 6 & 6.91 & 577 & $\begin{array}{l}439(100), 533(43), 425(38), \\
329(22), 515(17)\end{array}$ & $439: 377,395$ & $\beta_{\mathrm{AB}}$ \\
\hline 7 & 7.34 & 577 & $289(100), 425(32), 439(14)$ & 289: $245,205,179$ & $\varepsilon_{\mathrm{AB}}$ \\
\hline \multicolumn{6}{|c|}{ Dehydrotricatechins } \\
\hline \multicolumn{6}{|c|}{$m / z 861$ (theoretical number of isomers: 324 ) } \\
\hline 20 & 5.38 & 861 & $\begin{array}{l}799(100), 817(82), 697(38), \\
779(32), 283(30), 325(30), \\
825(29), 653(28), 575(24)\end{array}$ & n.r. & $(\gamma / \delta-\gamma)_{\mathrm{AB}}$ \\
\hline 27 & 7.76 & 861 & $\begin{array}{l}\text { 680(100), 723(62), 735(36), } \\
529(28), 573(17)\end{array}$ & $680: 529$ & $(\delta-\gamma / \delta)_{\mathrm{AB}}$ \\
\hline 30 & 8.44 & 861 & $\begin{array}{l}\text { 680(100), 723(65), 735(42), } \\
\text { 699(26), 779(25), 801(20), } \\
423(20), 573(15)\end{array}$ & $680: 529,557,512$ & $\begin{array}{l}(\delta-\gamma / \delta)_{\mathrm{AB}} / \\
\delta_{\mathrm{AB}}-\alpha_{\mathrm{AC}}^{\dagger}\end{array}$ \\
\hline \multicolumn{6}{|c|}{$m / 2,863$ (theoretical number of isomers: 360 ) } \\
\hline \multirow[t]{3}{*}{9} & 2.63 & 863 & $\begin{array}{l}801(100), 827(100), 819(73), \\
575(60), 783(44)\end{array}$ & $\begin{array}{l}801: 765,695,555 \\
739\end{array}$ & $\begin{array}{l}(\gamma / \delta-\varepsilon)_{\mathrm{AB}} / \\
\beta_{\mathrm{AC}}-\varepsilon_{\mathrm{AB}}^{\dagger}\end{array}$ \\
\hline & & & & $\begin{array}{l}827: 539,765,629 \\
791\end{array}$ & \\
\hline & & & & $\begin{array}{l}819: 783,757,775 \\
765,693,485\end{array}$ & \\
\hline 10 & 2.74 & 863 & $\begin{array}{l}603(100), 819(78), 801(72), \\
557(65), 575(49), 585(47), \\
699(44), 369(42), 411(38)\end{array}$ & $\begin{array}{l}603: 541,411,369 \\
567,559\end{array}$ & $(\gamma-\varepsilon)_{\mathrm{AB}}$ \\
\hline 11 & 3.55 & 863 & $\begin{array}{l}725(100), 803(22), 781(20), \\
819(19), 695(18)\end{array}$ & $\begin{array}{l}725: 681,663,465 \\
417\end{array}$ & $(\gamma / \delta-\beta / \varepsilon)_{\mathrm{AB}}$ \\
\hline 12 & 3.68 & 863 & $\begin{array}{l}827(100), 781(97), 803(95), \\
819(82), 801(78), 699(68)\end{array}$ & n.r. & $(\beta / \varepsilon-\gamma)_{\mathrm{AB}}$ \\
\hline 18 & 5.09 & 863 & $\begin{array}{l}819(100), 781(88), 737(75), \\
801(65), 699(48), 803(55), \\
827(36), 695(36)\end{array}$ & 781: 699 & $(\delta-\beta / \varepsilon)_{\mathrm{AB}}$ \\
\hline 22 & 6.18 & 863 & $\begin{array}{l}725(100), 711(27), 425(27), \\
701(22), 449(24), 413(17), \\
819(16), 695(15), 407(10), \\
287(8)\end{array}$ & $725: 449,287,681$ & $\begin{array}{l}(\beta / \varepsilon-\delta)_{\mathrm{AB}} / \\
\alpha_{\mathrm{AC}}-\delta_{\mathrm{AB}}^{\dagger}\end{array}$ \\
\hline 23 & 6.42 & 863 & $\begin{array}{l}\text { 725(100), 711(70), 425(32), } \\
701(30), 449(30), 413(18)\end{array}$ & $725: 449,287,681$ & $\begin{array}{c}(\beta / \varepsilon-\delta)_{\mathrm{AB}} / \\
\alpha_{\mathrm{AC}}-\delta_{\mathrm{AB}}^{\dagger}\end{array}$ \\
\hline \multirow[t]{2}{*}{24} & 6.70 & 863 & $\begin{array}{l}575(100), 725(94), 711(70), \\
449(50), 803(37), 781(30), \\
425(26), 407(24), 682(18), \\
287(12)\end{array}$ & $\begin{array}{l}575: 449,437,531 \\
407,287\end{array}$ & $(\gamma / \delta-\varepsilon)_{\mathrm{AB}}$ \\
\hline & & & & $725: 287,449$ & \\
\hline 25 & 7.05 & 863 & $\begin{array}{l}\text { 725(100), 711(66), 707(40), } \\
682(40), 569(33)\end{array}$ & $\begin{array}{l}725: 587,569,557 \\
545,681,407\end{array}$ & $(\gamma / \delta-\beta / \varepsilon)_{\mathrm{AB}}$ \\
\hline
\end{tabular}


Structural characterization of dehydrocatechin oligomers by MS/MS

\begin{tabular}{|c|c|c|c|c|c|}
\hline $\begin{array}{l}\text { Peak } \\
\text { no. }\end{array}$ & $\begin{array}{l}\text { Retention } \\
\text { time (min) }\end{array}$ & $\begin{array}{c}{[\mathrm{M}-\mathrm{H}]^{-}} \\
m / z\end{array}$ & $\begin{array}{l}\mathrm{MS}^{2} \text { fragments } \\
m / z \text { (intensity) }\end{array}$ & $\mathrm{MS}^{3}$ fragments $m / z$ & $\begin{array}{c}\text { Tentative } \\
\text { annotation" }\end{array}$ \\
\hline 26 & 7.38 & 863 & $\begin{array}{l}737(100), 725(58), 682(47), \\
711(47), 719(41), 449(21), \\
407(21), 695(20), 575(15)\end{array}$ & $\begin{array}{l}737: 693,567,585 \\
675\end{array}$ & $(\delta-\varepsilon)_{\mathrm{AB}}$ \\
\hline 28 & 7.82 & 863 & $\begin{array}{l}725(100), 682(90), 711(80), \\
683(68), 573(18)\end{array}$ & $725: 545,587,681$ & $(\gamma / \delta-\beta / \varepsilon)_{\mathrm{AB}}$ \\
\hline 29 & 7.98 & 863 & $\begin{array}{l}725(100), 711(75), 682(40), \\
707(34), 559(23), 693(27), \\
697(26)\end{array}$ & $\begin{array}{l}725: 559,587,545 \\
569,573,557,407 \\
681\end{array}$ & $(\gamma / \delta-\beta / \varepsilon)_{\mathrm{AB}}$ \\
\hline \multicolumn{6}{|c|}{$m / z 865$ (theoretical number of isomers: 100 ) } \\
\hline 13 & 3.88 & 865 & $\begin{array}{l}\text { 805(100), 783(81), 727(46), } \\
701(44), 713(42), 821(35), \\
681(30), 709(28), 393(14)\end{array}$ & n.r. & $(\beta-\beta)_{\mathrm{AB}}$ \\
\hline 14 & 4.49 & 865 & $\begin{array}{l}713(100), 727(84), 805(72), \\
783(62), 821(40), 701(30), \\
393(5)\end{array}$ & $\begin{array}{l}\text { 713: 607, 545, 574, } \\
497\end{array}$ & $(\beta / \varepsilon-\beta)_{\mathrm{AB}}$ \\
\hline \multirow[t]{2}{*}{15} & 4.64 & 865 & $\begin{array}{l}713(100), 727(98), 709(80), \\
821(52), 805(50), 783(42), \\
681(32), 829(30), 393(24)\end{array}$ & $713: 669,677,453$ & $(\beta-\beta)_{\mathrm{AB}}$ \\
\hline & & & & $\begin{array}{l}727: 665,683,493 \\
447,259\end{array}$ & \\
\hline \multirow[t]{2}{*}{16} & 4.81 & 865 & $\begin{array}{l}727(100), 713(67), 425(23), \\
821(22), 681(19), 393(16)\end{array}$ & $\begin{array}{l}727: 575,439,665 \\
287,493,535\end{array}$ & $(\beta-\beta)_{\mathrm{AB}}$ \\
\hline & & & & $713: 651,669,563$ & \\
\hline 17 & 4.96 & 865 & $\begin{array}{l}727(100), 713(100), 821(43), \\
709(40), 829(28), 783(26), \\
681(25), 393(23)\end{array}$ & $\begin{array}{l}727: 575,683,665 \\
393,535\end{array}$ & $(\beta-\beta)_{\mathrm{AB}}$ \\
\hline 19 & 5.31 & 865 & $\begin{array}{l}727(100), 783(40), 821(36), \\
709(34), 805(33), 713(27), \\
829(25)\end{array}$ & 727: $691,683,665$ & $(\beta / \varepsilon-\beta / \varepsilon)_{\mathrm{AB}}$ \\
\hline \multirow[t]{2}{*}{21} & 5.64 & 865 & $\begin{array}{l}713(100), 727(98), 821(34), \\
425(30), 681(28), 393(24), \\
577(18)\end{array}$ & $713: 669,575$ & $(\beta-\beta)_{\mathrm{AB}}$ \\
\hline & & & & $727: 439,575,665$ & \\
\hline \multicolumn{6}{|c|}{ Dehydrotetracatechins * } \\
\hline \multicolumn{6}{|c|}{$m / z, 1147$ (theoretical number of isomers: 5832 ) } \\
\hline 32 & 2.71 & 1147 & $1089,985,1113,1029$ & n.r. & \\
\hline 56 & 8.44 & 1147 & $966,1009,1021,1087$ & n.r. & \\
\hline \multicolumn{6}{|c|}{$m / z 1149$ (theoretical number of isomers: 9720) } \\
\hline 38 & 4.65 & 1149 & $1011,1047,993,1089$ & $\begin{array}{l}1011: 745,569,967 \\
766,663,873,677\end{array}$ & \\
\hline 44 & 5.58 & 1149 & $\begin{array}{l}1087,1011,1047,1105,1063, \\
981\end{array}$ & n.r. & \\
\hline 50 & 6.63 & 1149 & $\begin{array}{l}1011,997,981,711,723,573 \\
861,968\end{array}$ & $\begin{array}{l}\text { 1011: 735, 724, 873, } \\
681\end{array}$ & \\
\hline 51 & 6.84 & 1149 & $\begin{array}{l}1011,968,861,723,529,711 \\
981\end{array}$ & $\begin{array}{l}1011: 830,849,676 \\
967,873,723\end{array}$ & \\
\hline 52 & 7.30 & 1149 & $1011,723,968,680,800,873$ & $\begin{array}{l}1011: 830,680,723 \\
873,885\end{array}$ & \\
\hline
\end{tabular}




\begin{tabular}{|c|c|c|c|c|c|}
\hline $\begin{array}{l}\text { Peak } \\
\text { no. }\end{array}$ & $\begin{array}{l}\text { Retention } \\
\text { time (min) }\end{array}$ & $\begin{array}{c}{[\mathrm{M}-\mathrm{H}]^{-}} \\
\mathrm{m} / \mathrm{z}\end{array}$ & $\begin{array}{l}\mathrm{MS}^{2} \text { fragments } \\
m / z \text { (intensity) }\end{array}$ & MS $^{3}$ fragments $m / z$ & $\begin{array}{l}\text { Tentative } \\
\text { annotation }\end{array}$ \\
\hline 53 & 7.62 & 1149 & $1011,968,861,680,1089$ & $968: 830,817,798$ & \\
\hline 57 & 8.52 & 1149 & $\begin{array}{l}1011,1066,967,1022,1089, \\
766\end{array}$ & n.r. & \\
\hline 58 & 8.61 & 1149 & $1011,1023,1101,969,695$ & n.r. & \\
\hline 59 & 8.76 & 1149 & $585,447,1011,1089,968$ & n.r. & \\
\hline \multicolumn{6}{|c|}{$m / z 1151$ (theoretical number of isomers: 5400 ) } \\
\hline 31 & 2.02 & 1151 & $\begin{array}{l}1107,1091,1069,1116,987, \\
905\end{array}$ & n.r. & \\
\hline 34 & 3.24 & 1151 & $\begin{array}{l}1107,999,1091,1089,1064, \\
1115\end{array}$ & n.r. & \\
\hline 35 & 3.56 & 1151 & $1107,999,1050,903,981$ & n.r. & \\
\hline 36 & 4.02 & 1151 & $\begin{array}{l}1013,1069,1091,1061,984, \\
1111\end{array}$ & n.r. & \\
\hline 37 & 4.34 & 1151 & $\begin{array}{l}1013,999,1107,1091,969,725, \\
875\end{array}$ & n.r. & \\
\hline 39 & 4.83 & 1151 & $1107,999,863,861,1013$ & $\begin{array}{l}\text { 1107: } 1063,819 \\
1045,737,690\end{array}$ & \\
\hline 40 & 4.94 & 1151 & $1013,1107,999,970,725,1091$ & n.r. & \\
\hline 41 & 5.10 & 1151 & $\begin{array}{l}1013,1107,1091,862,557,805 \\
905,983\end{array}$ & n.r. & \\
\hline 42 & 5.25 & 1151 & $\begin{array}{l}999,1107,903,1013,1115, \\
1089,557,1045\end{array}$ & $\begin{array}{l}999: 937,964,735 \\
426,919,674\end{array}$ & \\
\hline & & & & 1013: $875,787,639$ & \\
\hline 43 & 5.43 & 1151 & $863,1107,1013,999,737$ & $863: 737,682,719$ & \\
\hline 45 & 5.67 & 1151 & $1013,999,995,1091,863$ & $\begin{array}{l}\text { 1013: } 875,969,843 \\
951\end{array}$ & \\
\hline \multirow[t]{2}{*}{46} & 5.83 & 1151 & $1013,999,981,725,713$ & 1013: 875 & \\
\hline & & & & $\begin{array}{l}999: 861,955,585, \\
365,735\end{array}$ & \\
\hline 47 & 6.04 & 1151 & $1013,999,1025,695,743$ & 1013: 833,875 & \\
\hline 48 & 6.16 & 1151 & $\begin{array}{l}1013,999,995,847,1089,636 \\
983\end{array}$ & $\begin{array}{l}\text { 1013: } 875,969,695 \\
951,829\end{array}$ & \\
\hline 49 & 6.46 & 1151 & $1013,999,695,725,1025,861$ & $\begin{array}{l}\text { 1013: } 861,695,777, \\
655\end{array}$ & \\
\hline 54 & 7.73 & 1151 & $969,1013,971,999,861$ & $969: 831,818,785$ & \\
\hline 55 & 7.84 & 1151 & $999,1013,970$ & 999: $818,861,955$ & \\
\hline \multicolumn{6}{|c|}{$m / z 1153$ (theoretical number of isomers: 1000 ) } \\
\hline 33 & 3.05 & 1153 & 1093, 1071, 1103, 919, & n.r. & \\
\hline
\end{tabular}

* The abbreviated nomenclature was used. When the interflavanic configuration is ambiguous, all options are noted, separated by " / ", † both the 'all-DhC' and the DhC-PC hybrid options are shown in the table, $\ddagger$ fragments are shown in order from high to low intensity, intensity values were not given, as annotation of the interflavanic configuration type was not possible, n.r.: $\mathrm{MS}^{3}$ data not recorded. 


\subsubsection{Signature fragments for the different interflavanic configurations in dehydrodicatechins}

To distinguish the different $\mathrm{DhC}_{2}$ isomers, it is important to specify signature fragments from their $\mathrm{MS}^{2}$ spectra. The $\mathrm{MS}^{2}$ fragments of the $\mathrm{DhC}_{2} \mathrm{~S}$ with $\mathrm{m} / \mathrm{z} 577$, either EC- $\beta_{\mathrm{AB}}-\mathrm{EC}$ or EC- $\varepsilon_{\mathrm{AB}}-\mathrm{EC}$, were compared in Table 4.2. Two different sets of compounds could be distinguished by characteristic fragments. The set of compounds 1-4 and $\mathbf{6}$ (when zooming in on fragments), and the set of compounds 5 and 7, showed a high intensity of the fragment $\mathrm{m} / \mathrm{z} 393$ or $\mathrm{m} / \mathrm{z} 289$, respectively. Two $\mathrm{MS}^{2}$ spectra, representative for each set, the isomers $\mathbf{3}$ and 5, are shown in Figure 4.5. The fragment of $\mathrm{m} / \mathrm{z} 289$ (interflavanic cleavage (Fig. 4.5B)) was clearly found for compound 5, whereas the fragment of $m / z 393$ (water loss followed by RDA fragmentation (Fig. 4.5A)) appeared only in the spectrum of compound 3. It is known that ether-linked catechin subunits in DhCs give higher abundance of the $m / z 289$ fragment than C-C linked catechin subunits. ${ }^{8}$ Therefore, $m / z 289$ is a signature fragment for annotation of $\mathrm{EC}-\varepsilon_{\mathrm{AB}}-\mathrm{EC}$. As the interflavanic configurations are annotated depending on the intensity of signature fragments, it is proposed to use the ratio of the intensities of $m / z 393: m / z 289$ for distinguishing EC- $\beta_{A B}-E C$ and $E C-\varepsilon_{A B}-E C$. If this ratio is $>1$, then the compound should be annotated as a EC- $\beta_{\mathrm{AB}}-\mathrm{EC}$. If this ratio is $<1$, then the compound should be annotated as $\mathrm{EC}-\varepsilon_{\mathrm{AB}}-\mathrm{EC}$.

Only one $\mathrm{DhC}_{2}$ was found with $\mathrm{m} / \mathrm{z} 575$ in the incubation (Fig. 4.4). It was known that $\mathrm{DhC}_{2} \mathrm{~s}$ with $\mathrm{m} / \mathrm{z} 575$ are mainly formed at $\mathrm{pH}$ values higher than $5.5 .^{8}$ Therefore, the incubation of EC with tyrosinase was performed at $\mathrm{pH} 8.0$ as well. Figure 4.6 shows the $\mathrm{MS}^{2}$ fragmentation spectra of two $\mathrm{DhC}_{2} \mathrm{~S}$ with $\mathrm{m} / \mathrm{z} 575$ formed in this incubation, which represented the two characteristic $\mathrm{MS}^{2}$ patterns that can be obtained.

To distinguish the two different interflavanic configurations with $m / z 575$, EC- $\gamma_{\mathrm{AB}}-\mathrm{EC}$ and $\mathrm{EC}-\delta_{\mathrm{AB}}-\mathrm{EC}$, the $\mathrm{MS}^{2}$ fragments were compared (Table 4.2). A fragment of $m / z 411$ was only found for the DhC shown in Figure 4.6B. This fragment could only be formed when an extra intraflavanoic ether bond is present between the C-ring and B-ring, as for EC- $\gamma_{\mathrm{AB}^{-}}$ EC (Fig. 4.1B). Fragments that appeared in the other spectra, in Figure 4.6A as well as for compound 8, were $m / z 407$ (RDA fragment), $m / z 437$ (RDA fragment) and the predominant $\mathrm{m} / \mathrm{z} 449$ (water loss followed by loss of B-ring). The proposed ratio of intensities for distinguishing $\mathrm{EC}-\gamma_{\mathrm{AB}}-\mathrm{EC}$ and $\mathrm{EC}-\delta_{\mathrm{AB}}-\mathrm{EC}$ is that of $m / z 449: m / z 411$. If this ratio is $>1$, then the compound should be annotated as a $E C-\delta_{\mathrm{AB}}-\mathrm{EC}$. If this ratio is $<1$, then the compound should be annotated as a $\mathrm{EC}-\gamma_{\mathrm{AB}}-\mathrm{EC}$.

With the annotation ratios described above, the various $\mathrm{DhC}_{2} \mathrm{~S}$ obtained after treatment of EC with tyrosinase were annotated (Table 4.1). The set of compounds 1-4 and 6 appeared to be $\mathrm{EC}-\beta_{\mathrm{AB}}-\mathrm{EC}$, whereas the set of compounds 5 and 7 were $\mathrm{EC}-\varepsilon_{\mathrm{AB}}-\mathrm{EC}$. The $\mathrm{C}$ $\mathrm{C}$ linked $\mathrm{DhC}_{2} \mathrm{~s}$ eluted earlier in the chromatogram than the ether-linked DhCs, indicating a 
higher polarity of the former ones. This might be explained by the loss of a free hydroxyl group upon formation of the ether bond. Finally, compound $\mathbf{8}$ was annotated as $\mathrm{EC}-\delta_{\mathrm{AB}}-\mathrm{EC}$.

Positional isomers of compounds with a particular interflavanic configuration could not be distinguished by the $\mathrm{MS}^{2}$ fragments. The fragments were formed by cleavage of the Aring and C-ring, or by cleaving the newly formed bonds, both of which could not give information on the position of the cross-link in the aromatic rings.
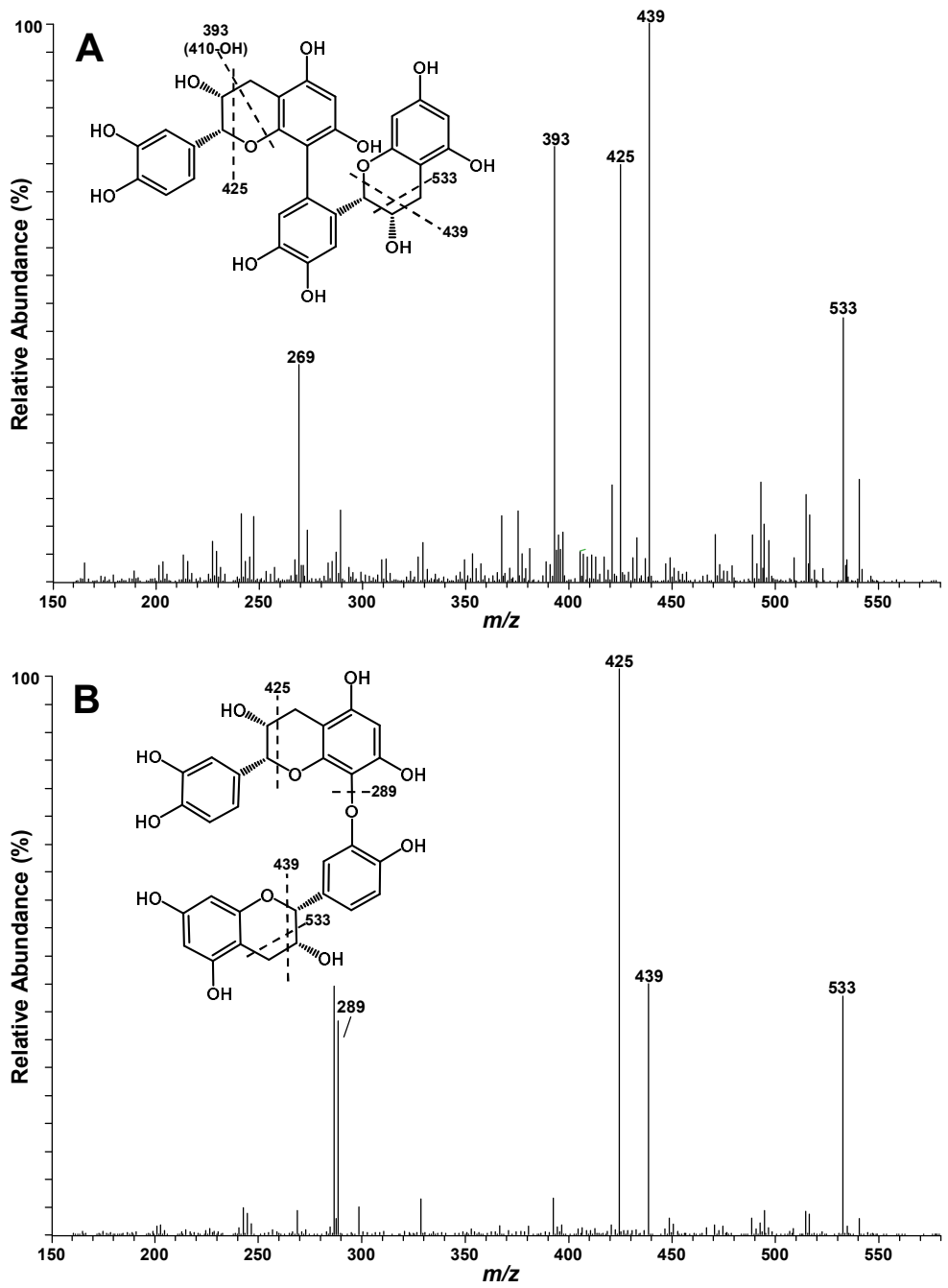

Figure 4.5 MS2 fragmentation spectra of two isomeric dehydrodicatechin structures with $\mathrm{m} / z 577$. (A) EC- $\beta_{\mathrm{AB}}-\mathrm{EC}$. (B) EC- $\varepsilon_{\mathrm{AB}}-\mathrm{EC}$. 

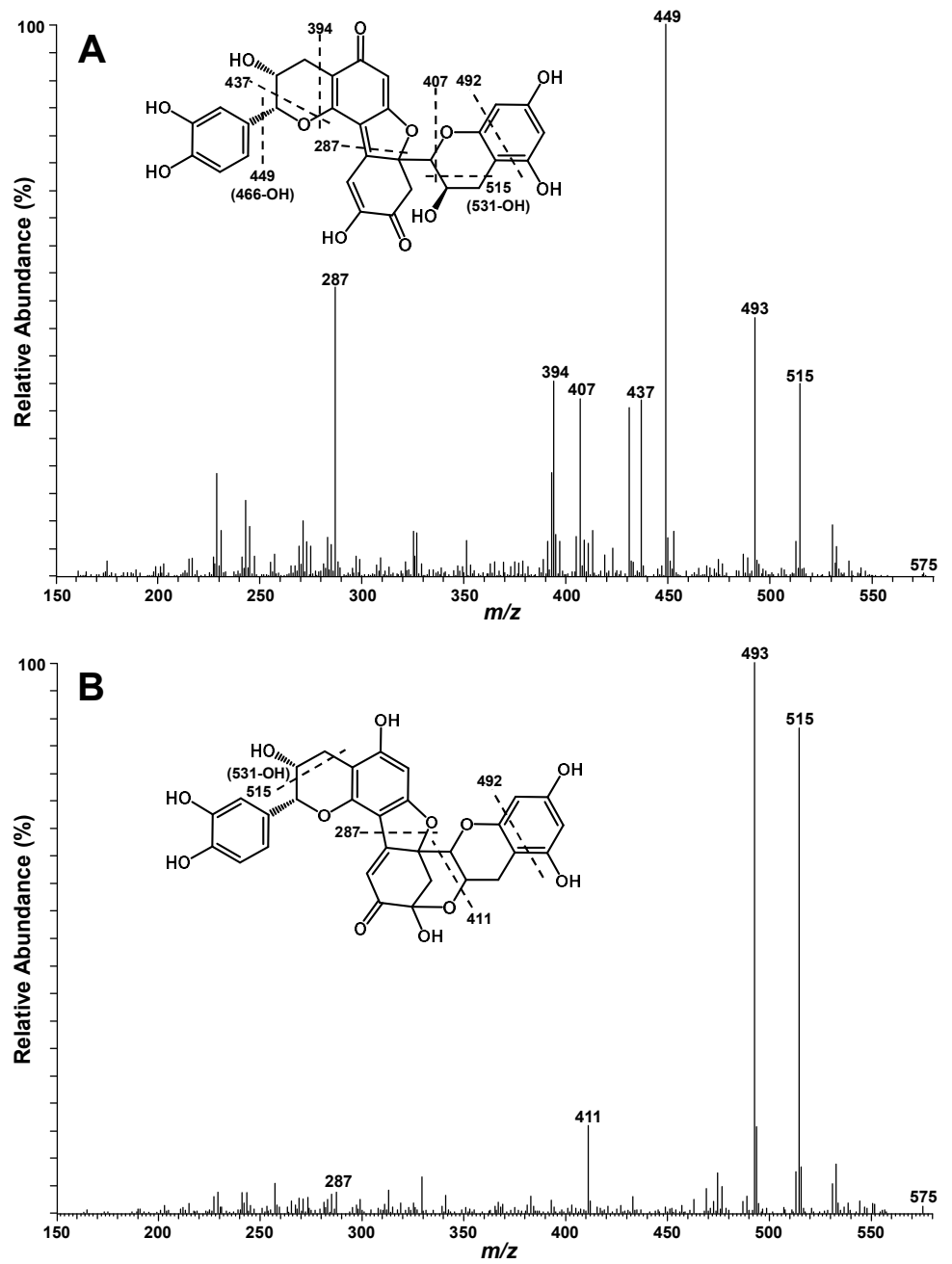

Figure 4.6 $\mathrm{MS}^{2}$ fragmentation spectra of two isomeric dehydrodicatechin structures with $\mathrm{m} /$ ₹ 575 . (A) $\mathrm{EC}-\delta_{\mathrm{AB}}-\mathrm{EC}$. (B) $\mathrm{EC}-\gamma_{\mathrm{AB}}-\mathrm{EC}$.

\subsubsection{Signature fragments to distinguish dehydrodicatechins from dimeric procyanidins}

As shown, the different isomeric interflavanic configurations of $\mathrm{DhC}_{2} \mathrm{~s}$ can be distinguished and annotated in model systems. Nevertheless, in black tea, they coexist with PCs, A-ring to C-ring linked isomers of DhCs. Therefore, it is important to specify signature fragments from both $\mathrm{MS}^{2}$ spectra, so that DhCs can be distinguished from PCs. 
An overview of the different fragments observed for the different $\mathrm{DhC}_{2} \mathrm{~s}$, compared with those of A-type and B-type PCs, is shown in Table 4.2.

Table 4.2 Intensities (\%) of fragments in the MS2 spectrum of the different DhC dimers and PC dimers. The fragments contributing to the signature ratio to distinguish the different interflavanic configurations are shaded green, the fragments of the signature ratio to distinguish DhCs from PC are shaded purple.

\begin{tabular}{|c|c|c|c|c|c|c|c|c|c|c|c|c|c|c|c|}
\hline \multicolumn{3}{|c|}{$\begin{array}{c}\text { Parent compound } \\
m / z 577\end{array}$} & \multicolumn{13}{|c|}{ Intensity of fragment $(\%)^{\dagger}$} \\
\hline & Fig. & Peak & 269 & 287 & 289 & 329 & 393 & 407 & 421 & 425 & 439 & 451 & 515 & 533 & 541 \\
\hline \multirow{7}{*}{ DhC } & 4.4A & 1 & & & & 31 & 92 & & 38 & & 100 & & 52 & 89 & 57 \\
\hline & 4.4A & 2 & & & & 39 & 100 & & 40 & 90 & 92 & & 59 & 96 & 58 \\
\hline & 4.4A & 3 & 33 & & & & 69 & & & 74 & 100 & & & 42 & \\
\hline & $4.4 \mathrm{~A}$ & 4 & 41 & & & & 95 & & & 97 & 100 & & & 54 & \\
\hline & $4.4 \mathrm{~A}$ & 5 & & 43 & 38 & & & & & 100 & 44 & & & 43 & \\
\hline & 4.4A & 6 & & & & 22 & 3 & & & 38 & 100 & & 17 & 43 & \\
\hline & 4.4A & 7 & & & 100 & & & & & 32 & 14 & & & & \\
\hline $\begin{array}{c}\text { B- } \\
\text { type } \\
\text { PC }\end{array}$ & S4.1A & $-{ }^{*}$ & & & 38 & & & 89 & & 100 & & 25 & & & \\
\hline
\end{tabular}

\begin{tabular}{|c|c|c|c|c|c|c|c|c|c|c|c|}
\hline \multicolumn{1}{|c|}{$\begin{array}{c}\text { Parent compound } \\
\boldsymbol{m} / \mathbf{5} 575\end{array}$} & \multicolumn{10}{|c|}{ Intensity of fragment (\%) } \\
& Fig. & Peak & $\mathbf{2 8 7}$ & $\mathbf{3 9 4}$ & $\mathbf{4 0 7}$ & $\mathbf{4 1 1}$ & $\mathbf{4 2 3}$ & $\mathbf{4 3 7}$ & $\mathbf{4 4 9}$ & $\mathbf{4 9 3}$ & $\mathbf{5 1 5}$ \\
\hline \multirow{3}{*}{ DhC } & $\mathbf{4 . 4 A}$ & $\mathbf{8}$ & 32 & 14 & 17 & & & 31 & 100 & & \\
\cline { 2 - 13 } & $\mathbf{4 . 6 A}$ & $-*$ & 54 & 35 & 30 & & & 30 & 100 & 45 & 34 \\
\cline { 2 - 13 } & $\mathbf{4 . 6 B}$ & $-*$ & & 6 & & 15 & & & & 100 & 87 \\
\hline $\begin{array}{c}\text { A- } \\
\text { type } \\
\text { PC }\end{array}$ & S4.1B & $-*$ & 30 & & 35 & & 100 & & 57 & & \\
\hline
\end{tabular}

tOnly intensities above $10 \%$ are shown.

* Chromatograms not shown, only MS² data.

When comparing the $\mathrm{MS}^{2}$ fragments of the $\mathrm{DhC}_{2} \beta\left(\mathrm{EC}-\beta_{\mathrm{AB}}-\mathrm{EC}\right)$ and $\mathrm{DhC}_{2} \varepsilon\left(\mathrm{EC}-\varepsilon_{\mathrm{AB}}-\right.$ EC) with B-type PC (EC- $\beta_{\mathrm{AC}}-\mathrm{EC}$ ) (Sup. Fig. S4.1A), all three $m / z$ 577, it was observed that the fragment $m / z 439$ (RDA fragment) was present in every $\mathrm{DhC}_{2}$ fragmentation pattern, but not in those of B-type PCs (Table 4.2). This was expected, because B-type PCs cannot 
form this fragment due to the absence of free AC-rings. ${ }^{8}$ Therefore, fragment $m / z 439$ can be used as signature fragment for $\mathrm{DhC}_{2} \mathrm{~s}$. In contrast, B-type PCs generated high intensity of a fragment with $m / z 407$ (RDA fragment) ${ }^{20}$ (Sup. Fig. S4.1A), which was present in intensities $<10 \%$ in the $\mathrm{MS}^{2}$ spectra of the $\mathrm{DhC}_{2}$ s (Table 4.2). The proposed ratio of the intensities for distinguishing $\mathrm{DhC}_{2}$ s from B-type PCs is that of $\mathrm{m} / \mathrm{z} 439: \mathrm{m} / \mathrm{z} 407$. A ratio > 1 indicates a $\mathrm{DhC}_{2}$, whereas a ratio $<1$ indicates a $\mathrm{B}$-type $\mathrm{PC}$.

The $\mathrm{MS}^{2}$ fragments of the $\mathrm{DhC}_{2} \gamma\left(\mathrm{EC}-\gamma_{\mathrm{AB}}-\mathrm{EC}\right), \mathrm{DhC}_{2} \delta\left(\mathrm{EC}-\delta_{\mathrm{AB}}-\mathrm{EC}\right)$ and A-type PC $\left(\mathrm{EC}-\alpha_{\mathrm{AC}}-\mathrm{EC}\right.$ ) (Sup. Fig. S4.1B), all three $m / z$ 575, were compared (Table 4.2). The fragment of $\mathrm{m} / \mathrm{z} 394$ (RDA fragment) was reported to be a fragment in $\mathrm{DhC}_{2} \mathrm{~s}$, which could not be formed from PCs. ${ }^{8}$ Even though this fragment was not a main fragment for all $\mathrm{DhC}_{2} \mathrm{~s}$, we propose to use it as signature fragment for $\mathrm{DhC}_{2} \mathrm{~s}$. The main fragment for A-type PC was $m / z 423$ (RDA fragment), which is not, or in low abundance $(<10 \%)$, found for $\mathrm{DhC}_{2} \mathrm{~s}$ (Table 4.2). Therefore, to distinguish between $\mathrm{DhC}_{2} \mathrm{~s}$ with $\mathrm{m} / z 575$ and A-type PCs, we propose to use the ratio of intensities of $m / z 394: m / z 423$. A ratio $>1$ indicates a $\mathrm{DhC}_{2}$, whereas a ratio $<1$, indicates an A-type $\mathrm{PC}$.

Both the ratio to distinguish DhCs from PCs and the ratio to distinguish the different interflavanic configurations are schematically summarized in a decision tree in Figure 4.7.

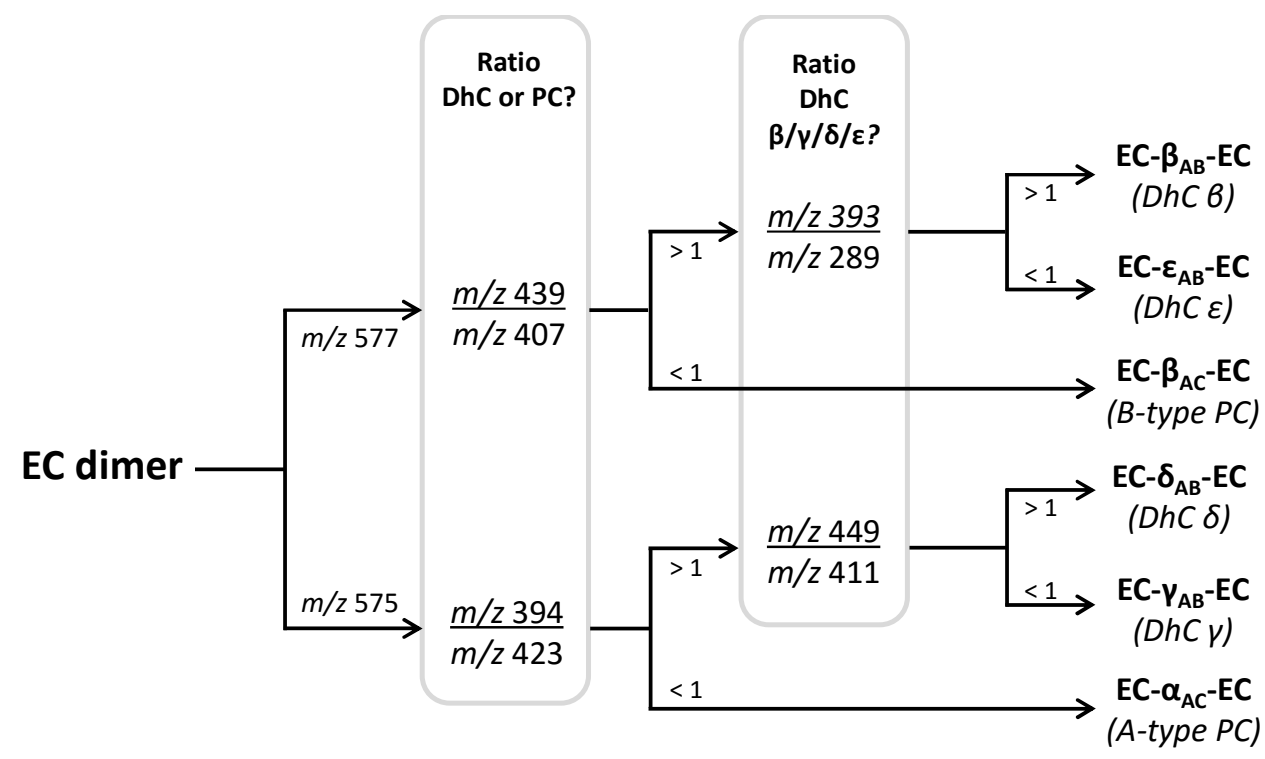

Figure 4.7 Decision tree for annotation of various isomeric dehydrodicatechins and dimeric procyanidins using the signature fragments formed in MS2. 


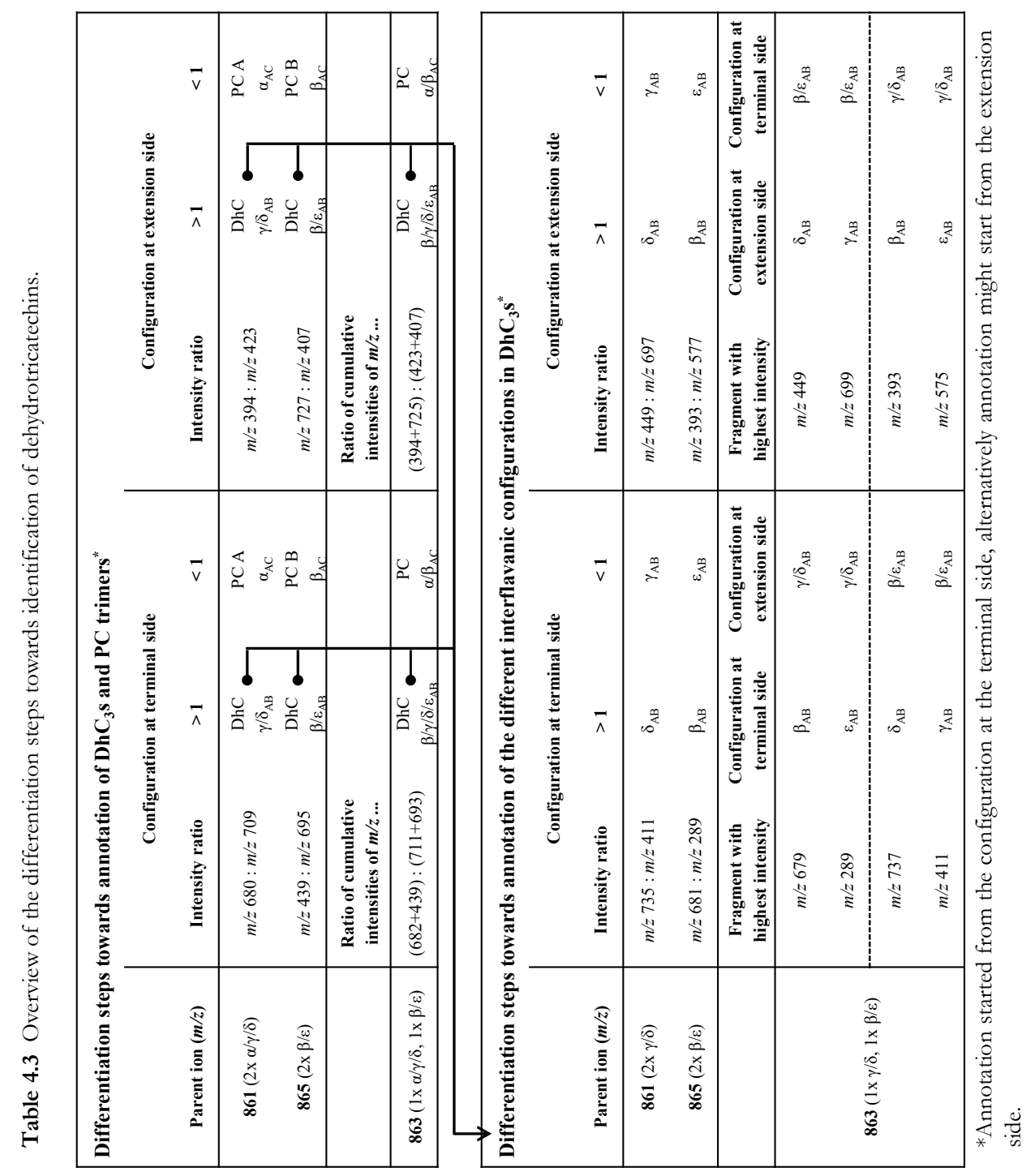




\subsubsection{Signature fragments for dehydrotricatechins}

The signature ratios determined for $\mathrm{DhC}_{2} \mathrm{~S}$ were extrapolated towards trimers. This is illustrated with the example of $\mathrm{DhC}_{2} \mathrm{EC}-\beta_{\mathrm{AB}}-\mathrm{EC}$, and $\mathrm{DhC}_{3} \mathrm{EC}-\beta_{\mathrm{AB}}-\mathrm{EC}-\beta_{\mathrm{AB}}-\mathrm{EC}$ (Sup. Fig. S4.2). $\mathrm{DhC}_{2} \mathrm{EC}-\beta_{\mathrm{AB}}-\mathrm{EC}$ yielded the signature fragments $m / z 439$ and $m / z 393 . \mathrm{A} \mathrm{DhC}_{3}(\mathrm{~m} / \mathrm{z}$ 865 ) with a $\beta_{\mathrm{AB}}$-configuration on the terminal side would yield the fragments of $m / z 439$ and $m / z 681(393+288)$, whereas a $\beta_{\mathrm{AB}}$-configuration on the extension side would yield the fragments of $m / z 727(439+288)$ and $m / z 393$ (Sup. Fig. S4.2). $\mathrm{DhC}_{3} \mathrm{~s}$ with $m / z 863$ possess two different sets of interflavanic configurations $(\beta / \varepsilon$ and $\gamma / \delta)$ combined in one molecule. To determine whether the $\beta / \varepsilon$ or the $\gamma / \delta$ configuration is on the terminal or extension side, the ratio of the cumulative intensity of both $\mathrm{DhC}$ signature fragments to that of the two fragments indicating PC configurations, were calculated, an overview of which is shown in Table 4.3.

Following the stepwise annotation towards $\mathrm{DhC}_{3} \mathrm{~s}$ (Table 4.3), the trimers formed in the incubation of EC with tyrosinase (Fig. 4.4B) can be tentatively annotated by their $\mathrm{MS}^{2}$ data (Table 4.1). It was expected that only $\mathrm{DhCs}$ would be formed upon enzymatic treatment. However, it cannot be excluded that DhC-PC hybrids were formed. The trimeric compounds 9, 22, 23 and $\mathbf{3 0}$ showed an $\mathrm{MS}^{2}$ fragment hinting at a $\mathrm{PC}$ configuration for one of the cross-links between the subunits, instead of a fragment of DhC. As tyrosinase is unable to oxidize the AC-rings, this might be explained by coupled oxidation, ${ }^{21}$ a redoxreaction where the EC-quinone reduces back to EC meanwhile oxidizing another compound. The EC-quinones, formed by tyrosinase, could have oxidized the AC-rings of the $\mathrm{DhC}_{2} \mathrm{~S}$ formed earlier, enabling the formation of $\mathrm{A} \rightarrow \mathrm{C}$ linkages.

Annotation of interflavanic configurations for DhCs with DP higher than 3 was not performed in this research, as the number of possibilities for fragmentation increases with size, and consequently the abundance of the individual fragments decreases. The fragmentation pattern became too complex and with too low signal intensities.

\subsubsection{Presence of dehydrocatechins in black tea}

Eight teas, differing in processing method and/or origin, were analysed for presence of $\mathrm{DhC}_{2} \mathrm{~S}$ and $\mathrm{DhC}_{3} \mathrm{~s}$ (Fig. 4.8). The samples were analysed with the MS/MS on selected ions method (Ch. 3). The $\mathrm{MS}^{2}$ data was analysed for the signature fragments, as established in this study, to distinguish DhCs and PCs. By making extracted ion chromatograms (EIC) of these signature fragments, it was found that PCs were present in at least 10 times higher concentrations than DhCs (Sup. Fig. S4.3). Nevertheless, the DhCs could be distinguished by their $\mathrm{MS}^{2}$ data, and their concentrations in the different teas are shown in Figure 4.8. Although the first annotation step of distinguishing DhCs from PCs (Fig. 4.7) was easily performed, despite the large difference in DhC and PC content, the annotation of the 
configuration of the DhCs was more challenging. Therefore, the DhCs with $m / z 577$ and $\mathrm{m} / \mathrm{z} 575$ might represent either the $\beta$ or $\varepsilon$-configuration and the $\gamma$ or $\delta$-configuration, respectively.

As can be seen from Figure 4.8, $\mathrm{DhC}_{2} \mathrm{~s} \beta / \varepsilon$ were present in 10 to 100 times higher concentrations than the $\mathrm{DhC}_{2} \mathrm{~s} \gamma / \delta$ or than the $\mathrm{DhC}_{3}$ s. Differences in the amounts of $\mathrm{DhC}_{2} \mathrm{~s}$ $\beta / \varepsilon$ were observed for the different teas, with the 'China black Yunnan' showing the highest concentration, whereas the 'Pu Ehr' tea (processed by exogenous microbial fermentation) showed a 10 times lower concentration of those compounds. The 'China black Yunnan' also showed a 2-10 times higher concentration of the $\mathrm{DhC}_{2} \mathrm{~s} \gamma / \delta$ than the other teas. The concentrations of the $\mathrm{DhC}_{3} \mathrm{~s}$ appeared to be similar in all the teas and, therefore, relatively high for the 'Pu Ehr' tea with respect to the $\mathrm{DhC}_{2} \mathrm{~s}$. From the $\mathrm{DhC}_{3} \mathrm{~s}, \mathrm{~m} / z 863$ was the most abundant in all teas, similar to the results found with the model system.

When comparing the EICs of the signature fragments of the DhCs (data not shown), the composition of DhCs could be compared per tea. Five clear $\mathrm{DhC}_{2} \mathrm{~s} \beta / \varepsilon$ could be distinguished for the different teas. Six of the teas had the same main $\mathrm{EC}-\varepsilon_{\mathrm{AB}}-\mathrm{EC}$ peak, whereas the 'China black Yunnan' and 'Pu Ehr tea' had a main EC- $\beta_{\mathrm{AB}}-\mathrm{EC}$ peak. The phenolic profiles of the Indian and Nepalese teas were quite similar, as were the phenolic profiles of the Chinese (except for the 'China black Yunnan' and 'Pu Ehr tea') and Taiwanese teas. The $\mathrm{DhC}_{2} \mathrm{~s} \gamma / \delta$ and $\mathrm{DhC}_{3} \mathrm{~s}$ did not give clear peaks in their EICs, because of the at least 10 times lower concentration than the $\mathrm{DhC}_{2} \mathrm{~s} \beta / \varepsilon$.

To determine the interflavanic configuration $(\beta, \gamma, \delta, \varepsilon)$ of the different $\mathrm{DhC}_{2} \mathrm{~s}$ in tea, the proposed ratios in Figure 4.7 could not be used as unambiguously as for model systems. Certain signature fragments that distinguish between interflavanic configurations of $\mathrm{DhCs}$, also occurred in the $\mathrm{MS}^{2}$ spectra of PCs. As PCs were present in at least 10 times higher concentrations than the DhCs in black tea, the balance in the intensity ratio of signature fragments is disturbed. Therefore, an extra step was needed for annotation. By comparing the EICs of the different signature fragments of the DhCs, tentative annotation of the interflavanic configurations was possible. The reasoning is explained by the example of the EC dimers with $m / z 577$ in the 'China black Yunnan' tea (Sup. Fig. S4.3).

Supplemental Figure S4.3A shows the $\mathrm{MS}^{2}$ chromatogram of the parent ion with $\mathrm{m} / \mathrm{z}$ 577. To distinguish $\mathrm{DhC}_{2} \mathrm{~S} \beta / \varepsilon$ and B-type PCs, an EIC of their signature fragment was made, $m / z 439$ for $\mathrm{DhC}_{2} \beta / \varepsilon$ (Sup. Fig. S4.3B) and $m / z 407$ for B-type PC (Sup. Fig. S4.3C). Two main peaks, and several lower peaks were shown for $\mathrm{DhC}_{2} \mathrm{~s} \beta / \varepsilon$. From the comparison of the intensities of EIC $\mathrm{m} / \mathrm{z} 439$ and $\mathrm{m} / \mathrm{z} 407$, it can be concluded that B-type $\mathrm{PCs}$ are at least 10 times more abundant than $\mathrm{DhC}_{2} \mathrm{~S} \beta / \varepsilon$. To determine the interflavanic configuration of the DhCs, EICs of the signature fragments for the types of interflavanic configurations were made, $m / z 393$ for a $\beta$-configuration (Sup. Fig. S4.3D) and $m / z 289$ for an $\varepsilon$-configuration (Sup. Fig. S4.3E). As the fragment of $m / z 393$ does not occur in B-type 
PCs (Table 4.2), it is obvious that all peaks visible in the EIC of $\mathrm{m} / z 393$ (Sup. Fig. S4.3D) should correspond to $\mathrm{DhC}_{2} \mathrm{~s} \beta$. The annotation of $\mathrm{DhC}_{2} \varepsilon$ was more challenging, as the fragment of $\mathrm{m} / \mathrm{z} 289$ also occurred in the fragmentation spectra of B-type PCs (Table 4.2). The EIC of $m / z 289$ (Sup. Fig. S4.3E) consequently corresponded mainly to the B-type PC peaks, because of their higher concentration. The $\mathrm{DhC}_{2} \varepsilon$ could be extrapolated by combining the patterns of three EICs (Sup. Fig. S4.3B, S4.3C and S4.3E), where the presence of a fragment with $\mathrm{m} / \mathrm{z} 439$ and $\mathrm{m} / \mathrm{z} 289$, combined with the absence of a fragment of $m / z$ 407, indicated a $\mathrm{DhC}_{2} \varepsilon$. The $\mathrm{DhC}_{2} \mathrm{~s} \varepsilon$ occurred at the end of the gradient (7.0-7.5 min), which was in accordance to the $\mathrm{DhC}_{2} \varepsilon$ found in the model system (Fig. 4.2).

Even though the PCs were present in at least 10 times higher concentrations compared to the DhCs, DhCs could be distinguished with their signature fragments. Hence, the presence of $\mathrm{DhC}_{2} \mathrm{~s}$ and $\mathrm{DhC}_{3} \mathrm{~s}$ in black tea has been established for the first time. Besides, the interflavanic configuration could be annotated in the complex black tea mixture. With this, we provide compelling evidence that the $\mathrm{DhC} \beta_{\mathrm{AB}}$ and $\mathrm{DhC} \varepsilon_{\mathrm{AB}}$ configurations were found as basic types of oligomerization in the 'oxidative cascade hypothesis', in addition to theaflavins, theasinensins and theacitrins. The rearrangement level could be extended with the $\mathrm{DhC} \gamma_{\mathrm{AB}}$ and $\delta_{\mathrm{AB}}$ linkages, besides theanaphtoquinones and theacoumarins.

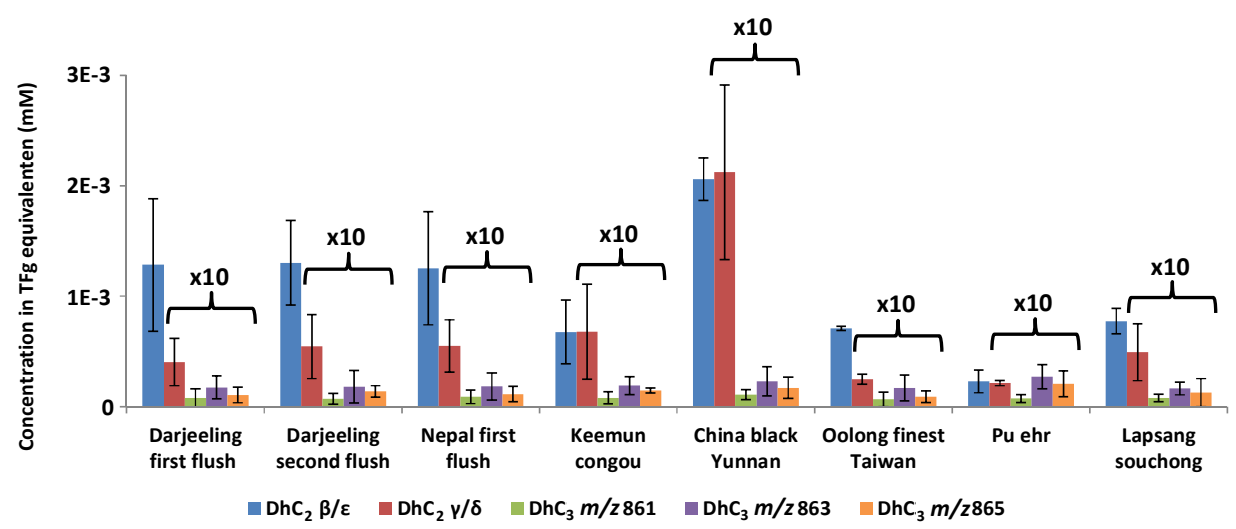

Figure 4.8 Content of DhC dimers and trimers (in $\mathrm{mM}$ TFg-equivalents; sum of various isomeric compounds) in different teas. For visual purposes, it is indicated which concentrations were multiplied by 10 .

\subsection{References}

1. Kuhnert, N.; Drynan, J. W.; Obuchowicz, J.; Clifford, M. N.; Witt, M. Mass spectrometric characterization of black tea thearubigins leading to an oxidative cascade hypothesis for thearubigin formation. Rapid Communications in Mass Spectrometry 2010, 24, 3387-3404. 
2. Tanaka, T.; Betsumiya, Y.; Mine, C.; Kouno, I. Theanaphthoquinone, a novel pigment oxidatively derived from theaflavin during tea-fermentation. Chemical Communications 2000, 1365-1366.

3. Kusano, R.; Matsuo, Y.; Saito, Y.; Tanaka, T. Oxidation mechanism of black tea pigment theaflavin by peroxidase. Tetrahedron Letters 2015, 56, 5099-5102.

4. Li, Y.; Shibahara, A.; Matsuo, Y.; Tanaka, T.; Kouno, I. Reaction of the black tea pigment theaflavin during enzymatic oxidation of tea catechins. Journal of Natural Products 2010, 73, 33-39.

5. Samanta, T.; Cheeni, V.; Das, S.; Roy, A. B.; Ghosh, B. C.; Mitra, A. Assessing biochemical changes during standardization of fermentation time and temperature for manufacturing quality black tea. Journal of Food Science and Technology 2015, 52, 2387-2393.

6. Cloughley, J. B.; Ellis, R. T. The effect of $\mathrm{pH}$ modification during fermentation on the quality parameters of central African black teas. Journal of the Science of Food and Agriculture 1980, 31, 924-934.

7. Verloop, A. J. W.; Gruppen, H.; Bisschop, R.; Vincken, J. P. Altering the phenolics profile of a green tea leaves extract using exogenous oxidases. Food Chemistry 2016, 196, 1197-1206.

8. Guyot, S.; Vercauteren, J.; Cheynier, V. Structural determination of colourless and yellow dimers resulting from (+)-catechin coupling catalysed by grape polyphenoloxidase. Phytochemistry 1996, 42, 1279-1288.

9. Drynan, J. W.; Clifford, M. N.; Obuchowicz, J.; Kuhnert, N. The chemistry of low molecular weight black tea polyphenols. Natural Product Reports 2010, 27, 417-462.

10. Nonaka, G.; Kawahara, O.; Nishioka, I. Tannins and related compounds. XV. A new class of dimeric flavan-3-ol gallates, theasinensins A and B, and proanthocyanidin gallates from green tea leaf. Chemical \& Pharmaceutical Bulletin 1983, 31, 3906-3914.

11. Fraser, K.; Harrison, S. J.; Lane, G. A.; Otter, D. E.; Hemar, Y.; Quek, S. Y.; Rasmussen, S. HPLC-MS/MS profiling of proanthocyanidins in teas: A comparative study. Journal of Food Composition and Analysis 2012, 26, 43-51

12. Kalili, K. M.; Villiers, A. d. Off-line comprehensive two-dimensional hydrophilic interaction $\mathrm{x}$ reversed phase liquid chromatographic analysis of green tea phenolics. Journal of Separation Science 2010, 33, 853863.

13. Sarracino, D.; Richert, C. Quantitative MALDI-TOF MS of oligonucleotides and a nuclease assay. Bioorganic \& Medicinal Chemistry Letters 1996, 6, 2543-2548.

14. Tang, K.; Allman, S. L.; Jones, R. B.; Chen, C. H. Quantitative analysis of biopolymers by matrix-assisted laser desorption. Analytical Chemistry 1993, 65, 2164-2166.

15. Mané, C.; Sommerer, N.; Yalcin, T.; Cheynier, V.; Cole, R. B.; Fulcrand, H. Assessment of the molecular weight distribution of tannin fractions through MALDI-TOF MS analysis of protein-tannin complexes. Analytical Chemistry 2007, 79, 2239-2248.

16. Thompson, R. S.; Jacques, D.; Haslam, E.; Tanner, R. J. N. Plant proanthocyanidins. Part I. Introduction the isolation, structure, and distribution in nature of plant procyanidins. Journal of the Chemical Society, Perkin Transactions 1 1972, 1387-1399.

17. Weinges, K.; Mattauch, H. Der chemische Konstitutionsbeweis des Dehydro-dicatechins A. ChemikerZeitung 1971, 95, 155-156.

18. Weinges, K.; Huthwelker, D. Isolierung und konstitutionsbeweis eines 8,6'-verknüpften dehydrodicatechins (B4). Liebig's Annalen der Chemie 1970, 731, 161-170.

19. Hemingway, R. W.; Foo, L. Y.; Porter, L. J. Linkage isomerism in trimeric and polymeric 2,3-cisprocyanidins. Journal of the Chemical Society, Perkin Transactions 1 1982, 1209-1216.

20. Appeldoorn, M. M.; Vincken, J. P.; Sanders, M.; Hollman, P. C. H.; Gruppen, H. Combined normal-phase and reversed-phase liquid chromatography/ESI-MS as a tool to determine the molecular diversity of A-type procyanidins in peanut skins. Journal of Agricultural and Food Chemistry 2009, 57, 6007-6013. 
21. Tanaka, T.; Mine, C.; Inoue, K.; Matsuda, M.; Kouno, I. Synthesis of theaflavin from epicatechin and epigallocatechin by plant homogenates and role of epicatechin quinone in the synthesis and degradation of theaflavin. Journal of Agricultural and Food Chemistry 2002, 50, 2142-2148.

\subsection{Supplementary data}
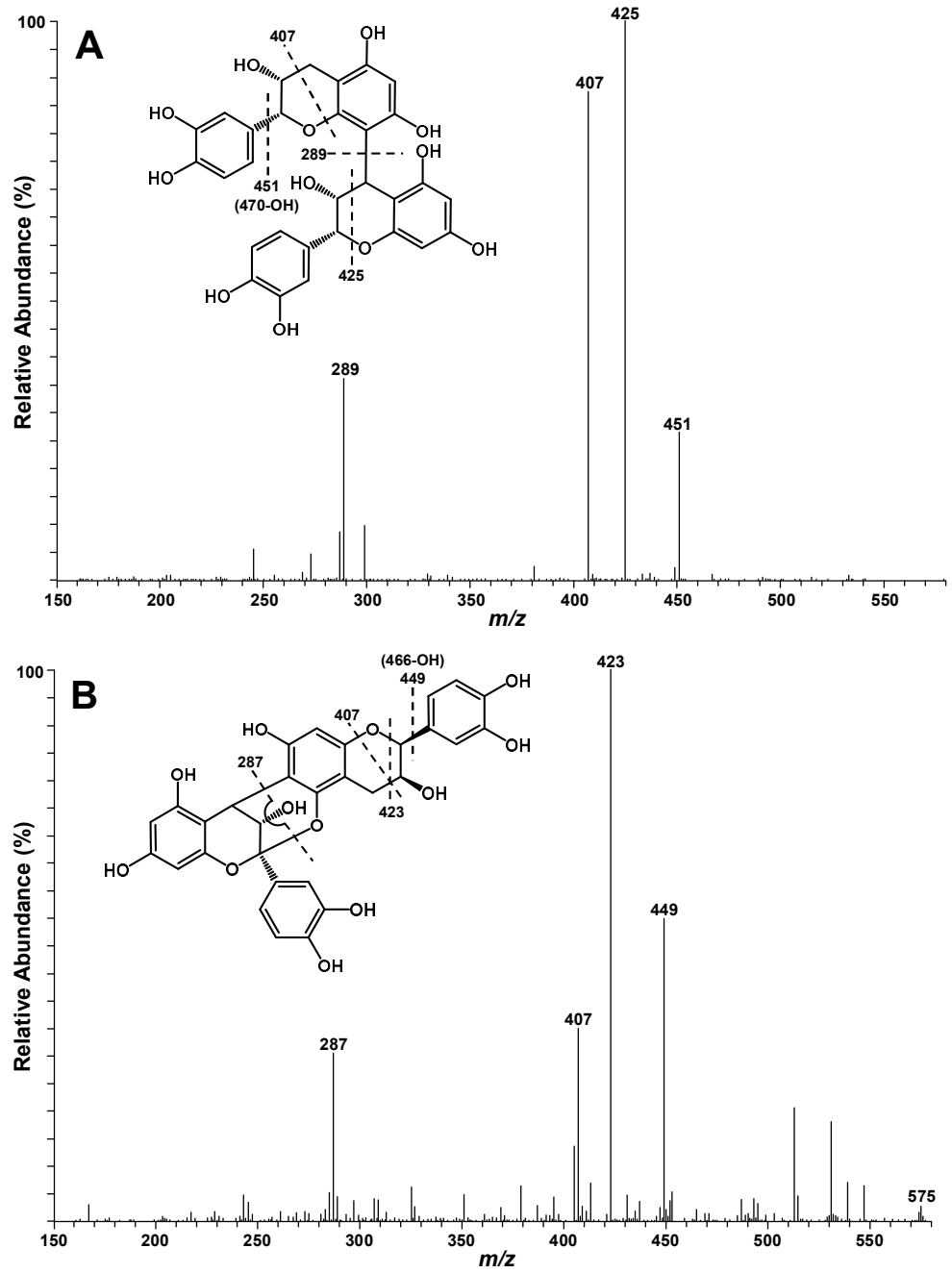

Figure S4.1 MS 2 fragmentation spectra from (A) a procyanidin B (EC- $\left.\beta_{\mathrm{AC}}-\mathrm{EC}\right)$, and $(\mathbf{B})$ a procyanidin A (EC- $\left.\alpha_{\mathrm{AC}}-\mathrm{EC}\right)$, from a procyanidin extract. 


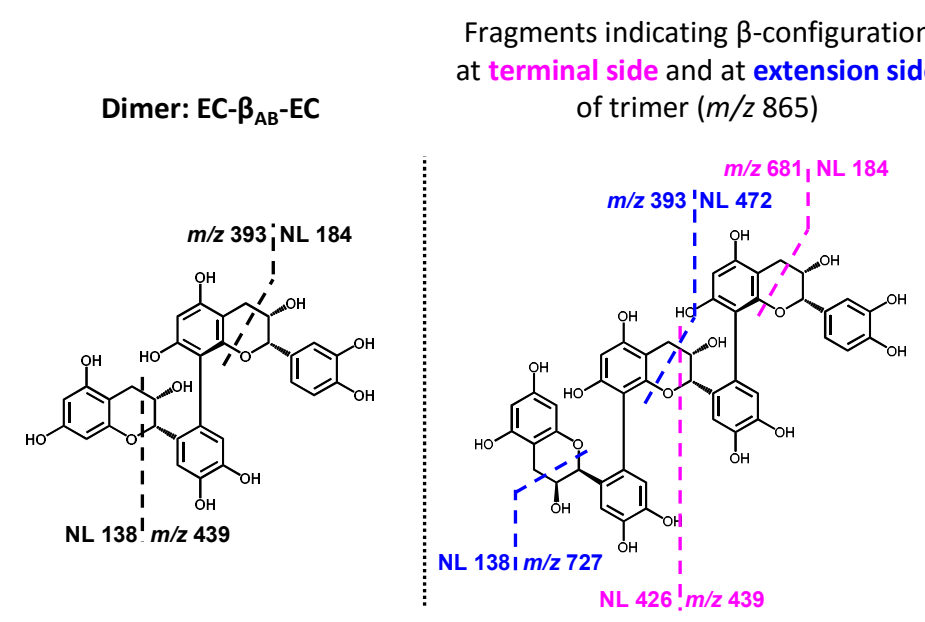

Figure S4.2 Schematic representation showing the extrapolation of the signature fragments determined for $\mathrm{DhC}_{2} \mathrm{~s}$ towards $\mathrm{DhC}_{3} \mathrm{~s}$.

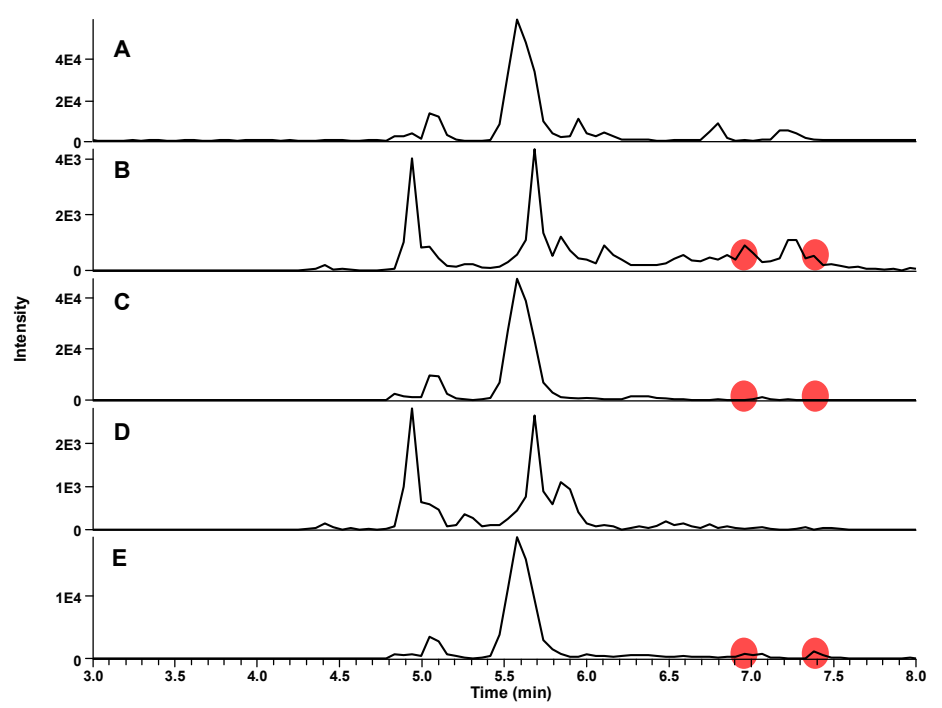

Figure S4.3 RP-UHPLC-MS chromatograms from a Chinese black Yunnan tea. A) MS/MS on selected ion $m / z 577$, B) EIC of fragment $m / z 439$ from MS/MS on selected ion $m / z 577$, C) EIC of fragment $m / z 407$ from MS/MS on selected ion $m / z 577$, D) EIC of fragment $m / z, 393$ from MS/MS on selected ion $m / z 577, \mathbf{E})$ EIC of fragment $m / z 289$ from MS/MS on selected ion $m / z$ 577. The red circles highlight the presence or absence of fragments, indicating an $\varepsilon$-configured $\mathrm{DhC}_{2}$. 


\title{
Chapter 5
}

\section{Peroxidase is responsible for the hydroxylation step in the 'oxidative cascade' during processing of black tea}

\begin{abstract}
Black tea characteristics as brown colour, bitter taste and astringent mouthfeel are largely determined by thearubigins, the formation of which is thought to involve at least two of the following three oxidation steps: (i) oligomerization, (ii) rearrangement, and (iii) hydroxylation. The first two steps are mainly catalysed by the polyphenol oxidase (PPO) catecholase, whereas the enzyme responsible for hydroxylation has not yet been identified. Two main enzyme activities, peroxidase (POD) and PPO, were shown to occur in tea leaves, the former of which was hypothesized to be responsible for hydroxylation. A model system with horseradish POD and mushroom tyrosinase was used to investigate hydroxylation of theaflavins (TF). POD was found capable of hydroxylation. TFs with up to five extra hydroxyl groups were annotated by LC-MS, using their $\mathrm{MS}^{2}$ data. Hydroxylation by POD was also shown for other oligomeric phenolics, such as theanaphtoquinones, theatridimensins and dehydrodicatechins. The $\mathrm{H}_{2} \mathrm{O}_{2}$ concentration influenced the extent of hydroxylation. At $0.01 \mathrm{mM} \mathrm{H}_{2} \mathrm{O}_{2}$ hydroxylation occurred, but at higher $\mathrm{H}_{2} \mathrm{O}_{2}$ concentration the extent of hydroxylation decreased. Similar compounds with extra hydroxyl groups were evidenced in a black tea, by using a selective screening method with RP-UHPLC-MS. TFs with up to five extra hydroxyl groups, and traces of other oligomeric catechins with extra hydroxyl groups, could be identified by their $\mathrm{MS}^{2}$ data in a commercial black tea, without any further sample pre-treatment.
\end{abstract}

Based on: Annewieke J.W. Verloop, Jean-Paul Vincken, Harry Gruppen. 2016 - To be submitted. 


\subsection{Introduction}

Black teas are known for their brown colour, bitter taste and astringent mouthfeel. These characteristics are largely determined by the phenolic profile of the tea. In black teas, this profile consists of oxidation products of catechins, the phenolics initially present in the green tea leaves. The catechins are enzymatically oxidized into mainly thearubigins (TR). $\mathrm{TR}$ is the collective noun for the complex phenolics present in a black tea. Oxidation of green tea leaves into black tea comprises different levels, which are described by the 'oxidative cascade hypothesis'. ' The different oxidation levels are (i) oligomerization, (ii) rearrangement, and (iii) hydroxylation. All reactions combined are considered to result in the extensive molecular diversity of TRs in black tea. ${ }^{l}$

The oligomerization and rearrangement levels have been extensively studied, and the substrates involved are largely known. ${ }^{2}$ The level of oligomerization can be divided into two types of oligomerization. (i) The basic oligomerization types, which can be formed from two subunits in one oxidation step: the theaflavin type (TF), ${ }^{3,4}$ the theasinensin type (TS), ${ }^{5-7}$ the theacitrin type (TC) $)^{8,9}$ and the dehydrocatechins (DhC) $\beta_{\mathrm{AB}}$ and $\varepsilon_{\mathrm{AB}}$ types $^{10}$ (Ch. 4). (ii) The tripartite oligomerization types, which require a preformed basic oligomerization between two catechin subunits serving as a scaffold onto which a third catechin is superimposed. An example of the latter is the theatridimensin type (T3D) ${ }^{11}(\mathbf{C h}$. 3). Both types of oligomers can also undergo intramolecular rearrangements when oxidized, in which the interflavanic configuration is altered. For instance, the theanaphtoquinone configuration (TNQ) ${ }^{12}$ and theacoumarin configuration (TCou) ${ }^{13}$ are formed from a TF type interflavanic configuration, whereas DhCs $\delta_{\mathrm{AB}}$ and $\gamma_{\mathrm{AB}}{ }^{10}$ configurations are formed from $\mathrm{DhC} \beta_{\mathrm{AB}}$.

The oxidation level of hydroxylation has not been given much attention yet. The occurrence of hydroxylation has been evidenced by extracted ion chromatograms and $\mathrm{MS}^{2}$ data of black tea fractions for several catechin dimers. ${ }^{14,15}$ It is unknown which enzyme from tea leaves is responsible for the actual hydroxylation. Preliminary evidence from model systems indicated that peroxidase (POD) might hydroxylate theaflavin gallates (TFg), but confirmation by $\mathrm{MS}^{2}$ fragment spectra was not provided. ${ }^{16}$

This research aims to determine which oxidative enzymes from tea leaves are responsible for the different oxidation levels in the 'oxidative cascade hypothesis'. In tea leaves, polyphenol oxidase (PPO) and POD activities are present, POD in 5 times higher concentration than PPO. ${ }^{I 7}$ PPO activity is mostly associated with formation of TFs, whereas POD activity has been associated with subsequent oxidation of TFs into TRs. ${ }^{17-19}$ However, it has not been specified in which of the oxidation reactions of the 'oxidative cascade hypothesis' POD is involved. 
It is hypothesized that POD is the main enzyme responsible for the hydroxylation reaction of TR formation in black tea. In this research, model systems are used with subsets of complex tea phenolics: TF, TNQ, T3D, DhC $\beta_{\mathrm{AB}} / \varepsilon_{\mathrm{AB}}$ and DhCs $\delta_{\mathrm{AB}} / \gamma_{\mathrm{AB}}$. To be able to characterize the contribution of POD to the hydroxylation, a pure enzyme extract should be used. As oxidative enzymes from tea leaves are not available individually, a POD from horseradish was used. A tyrosinase from mushroom, representing PPO activity, was used as a comparison to POD.

\subsection{Materials and Methods}

\subsubsection{Materials}

(-)-Epicatechin (EC), (-)-epigallocatechin gallate (EGCg), theaflavin-3-gallate (TFg), sodium chloride ( $\mathrm{NaCl}$ ), polyvinylpolypyrrolidone (PVPP), 2,2'-azino-bis(3ethylbenzothiazoline-6-sulphonic acid (ABTS), L-ascorbic acid, 30\% (w/w) hydrogen peroxide solution, peroxidase from horseradish and mushroom tyrosinase were purchased from Sigma Aldrich (St. Louis, MO, USA). L-Tyrosine was purchased from Fluka (Sigma Aldrich), disodium hydrogen phosphate, sodium dihydrogen phosphate, sodium acetate and anhydrous acetic acid from Merck (Darmstadt, Germany), and anhydrous citric acid from Acros Organics (Geel, Belgium). (-)-Epigallocatechin (EGC) was prepared from EGCg as described elsewhere. ${ }^{20}$ Ultra-high-performance liquid chromatography mass spectrometry (UHPLC-MS) grade acetonitrile (ACN) was obtained from Biosolve (Valkenswaard, The Netherlands). Water was prepared using a Milli-Q water purification system (Millipore, Billerica, MA, USA). Fresh tea leaves (Camellia sinensis var. assamica) from Kenya were kindly provided by Unilever R\&D (Vlaardingen, The Netherlands). A black Chinese Yunnan tea was obtained from a local store.

\subsubsection{Enzyme extraction}

Frozen tea leaves were blended for $1 \mathrm{~min}$ in a blender ( $400 \mathrm{~W}$, Waring, Stamford, CT, USA) with $10 \mathrm{mM}$ sodium acetate buffer $\mathrm{pH} 6.0$ containing PVPP and $0.2 \mathrm{M} \mathrm{NaCl}$, using a weight based ratio of leaves : PVPP : buffer solution of 1:1:10. The pulp obtained was centrifuged $\left(10 \mathrm{~min}, 8,000 \mathrm{~g}, 10^{\circ} \mathrm{C}\right)$, and subsequently filtered through a Büchner funnel using filter paper (Grade 1, Whatman, Chalfont St. Giles, UK). The filtrate was centrifuged (10 min, $\left.8,000 \mathrm{~g}, 10^{\circ} \mathrm{C}\right)$. The supernatant obtained is referred to as 'enzyme extract' and used for activity measurements. 


\subsubsection{Oxidative enzyme activity}

Two hundred $\mu \mathrm{L}$ of enzyme extract was combined with $100 \mu \mathrm{L}$ of a $3 \mathrm{mM}$ substrate solution in a 96-wells plate. In case of tyrosine, a $2.5 \mathrm{mM}$ substrate solution was used. Substrate solutions were prepared in $100 \mathrm{mM}$ sodium phosphate buffer $\mathrm{pH}$ 5.5. PPO activity was measured using EC as substrate, combined PPO with POD activity was measured using EC $+0.01 \% \mathrm{H}_{2} \mathrm{O}_{2}$, laccase activity was measured using ABTS as substrate, and cresolase activity was measured using tyrosine as substrate. Absorbance at $420 \mathrm{~nm}$, or $520 \mathrm{~nm}$ for tyrosine, was monitored in time for $30 \mathrm{~min}$. Reaction rates were calculated from the slopes of the linear segment of the absorbance versus time curve.

\subsubsection{Preparation of material enriched in oligomerized catechins}

Materials enriched in theaflavin (TF), theatridimensin (T3D), theanaphtoquinone (TNQ) and dehydrocatechins $(\mathrm{DhC})$ were prepared by an incubation of $\mathrm{EC}+\mathrm{EGC}$ with tyrosinase. $\mathrm{EC}$ and EGC were equimolarly used as substrates at a total concentration of $1 \mathrm{mM}$. Tyrosinase $(0.0755 \mathrm{U} / \mathrm{mg}$ ) was added to a final concentration of $0.001 \mathrm{U} / \mathrm{mL}$ ( 1 unit $=$ the amount of enzyme that converts $1 \mu \mathrm{mol}$ of oxygen per min with catechin at $\mathrm{pH} 5.5$ and 25 $\left.{ }^{\circ} \mathrm{C}\right)$. Incubations were performed in citric acid - sodium phosphate buffer $(30 \mathrm{mM}) \mathrm{pH} 5.5$ for the TF, TNQ and T3D pool, and a sodium phosphate buffer $(10 \mathrm{mM}) \mathrm{pH} 8.0$ for the dehydrodicatechin pools. The reaction flask was incubated in the dark at $25{ }^{\circ} \mathrm{C}$ under continuous stirring. After one hour the reaction was stopped by adding $20 \mu \mathrm{L}$ of saturated ascorbic acid per $\mathrm{mL}$ reaction mixture. The amount of ascorbic acid added was sufficient to arrest the reaction for at least $12 \mathrm{~h}$. Subsequently, the sample was treated by solid-phase extraction (SPE) using a $10 \mathrm{~g}$ C18 Sep-Pak column washed and eluted with water and $\mathrm{MeOH}$, according to the instructions of the manufacturer (Waters, Milford, MA, USA). The $\mathrm{MeOH}$ fraction was evaporated, dissolved in water, and freeze-dried. The freeze-dried material was dissolved in water at a concentration of $\sim 3 \mathrm{mg} / \mathrm{mL}$. Subsequently, the material was fractionated by Flash chromatography, using a $4 \mathrm{~g}$ Reveleris C18 column on a Reveleris Flash system (Grace, Deerfield, IL, USA) operated at $15 \mathrm{~mL} / \mathrm{min}$. Water containing $1 \%(\mathrm{v} / \mathrm{v}) \mathrm{ACN}$ and $0.1 \%(\mathrm{v} / \mathrm{v})$ acetic acid (eluent $\mathrm{A}$ ) and $\mathrm{ACN}$ containing $0.1 \%$ $(\mathrm{v} / \mathrm{v})$ acetic acid (eluent B) were used as eluents. The following elution profile was used: 0 $1 \mathrm{~min}$, isocratic on $0 \% \mathrm{~B} ; 1-16 \mathrm{~min}$, linear gradient from $0-50 \% \mathrm{~B} ; 16-18 \mathrm{~min}$, linear gradient from $50-100 \% \mathrm{~B} ; 18-21 \mathrm{~min}$, isocratic on $100 \% \mathrm{~B}$. Fractions were collected and analysed by RP-UHPLC-MS as described below. Fractions were pooled to yield pools enriched in TF ( 95\%), T3D ( 35\%), TNQ ( 25\%), DhC $\beta_{\mathrm{AB}}$ and $\varepsilon_{\mathrm{AB}}(\sim 35 \%)$ or DhC $\gamma_{\mathrm{AB}}$ and $\delta_{\mathrm{AB}}(\sim 10 \%)$, with the percentage purity based on UV absorbance at $270 \mathrm{~nm}$, and the annotation based on MS data. The pooled fractions were freeze-dried and stored at $-20^{\circ} \mathrm{C}$. During the preparation steps the samples were kept from light. 


\subsubsection{Oligomerized catechin incubations with POD or tyrosinase}

Pools enriched in TF, T3D, TNQ, DhCs $\beta_{\mathrm{AB}} / \varepsilon_{\mathrm{AB}}$ and $\mathrm{DhC} \gamma_{\mathrm{AB}} / \delta_{\mathrm{AB}}$ were used as substrates for subsequent hydroxylation reactions at an approximate concentration of 0.1 $\mathrm{mM}$. POD was added to a final concentration of $0.004 \mathrm{U} / \mathrm{mL}$ ( 1 unit $=$ the amount of enzyme that converts $1 \mu \mathrm{mol}$ of pyrogallol into purpurogallin per min at $\mathrm{pH} 6.0$ and $20^{\circ} \mathrm{C}$ ) or tyrosinase was added to a final concentration of $0.001 \mathrm{U} / \mathrm{mL}$. Incubations were performed in citric acid - sodium phosphate buffer $(30 \mathrm{mM}) \mathrm{pH}$ 5.5. Hydrogen peroxide was added to a final concentration of $0.1 \mathrm{mM}$, or in various final concentrations $(0,0.01$, $0.025,0.05,0.1,0.15,0.2$ and $0.25 \mathrm{mM})$, when specified. Samples $(100 \mu \mathrm{L})$ were incubated at $25^{\circ} \mathrm{C}$ for $0,5,10,15,20,25,30,45,60,90$ and $120 \mathrm{~min}$, after which the reaction was stopped by adding $2 \mu \mathrm{L}$ of freshly prepared saturated ascorbic acid solution. The samples were centrifuged $\left(10,000 \mathrm{~g}, 5 \mathrm{~min}, 20^{\circ} \mathrm{C}\right)$ prior to analysis by RP-UHPLC-MS. Compounds were identified and semi-quantified by mass spectrometry.

\subsubsection{Extraction of black tea phenolics}

Black tea $(0.5 \mathrm{~g})$ was three times extracted with $50 \mathrm{~mL}$ boiling water, for $10 \mathrm{~min}$ under continuous stirring. Extracts were filtered through paper filter (Grade 1, Whatman) by Büchner filtration and the filtrates were combined. The samples were centrifuged $(10,000 \mathrm{~g}$, 5 min, $20^{\circ} \mathrm{C}$ ) prior to analysis by RP-UHPLC-MS.

\subsubsection{RP-UHPLC analysis}

Samples were analysed on an Accela UHPLC system (Thermo Scientific, San Jose, CA, USA) equipped with a pump, an auto sampler at $15{ }^{\circ} \mathrm{C}$ and a photodiode array (PDA) detector. Samples $(2 \mu \mathrm{L})$ were injected onto a Hypersil Gold column $(2.1 \mathrm{x} 150 \mathrm{~mm}$, particle size of $1.9 \mu \mathrm{m}$, Thermo Scientific). Water containing $1 \%(\mathrm{v} / \mathrm{v}) \mathrm{ACN}$ and $0.1 \%(\mathrm{v} / \mathrm{v})$ acetic acid (eluent A) and ACN containing $0.1 \%(\mathrm{v} / \mathrm{v})$ acetic acid (eluent B) were used as eluents. The flow rate was $300 \mu \mathrm{L} / \mathrm{min}$, and the column oven temperature was controlled at $30{ }^{\circ} \mathrm{C}$. The PDA detector was set to measure 200-600 $\mathrm{nm}$. The following elution profile was used: 0-1 min, isocratic on 5\% (v/v) B; 1-12 min, linear gradient from 5-50\% (v/v) B; 12-13 min, linear gradient from 50-100\%; 13-18 min, isocratic on $100 \%(\mathrm{v} / \mathrm{v}) \mathrm{B} ; 18-18.1$ min, linear gradient from 100-5\% (v/v) B; and 18.1-22 min, isocratic on 5\% (v/v) B. 


\subsubsection{Electrospray Ionization - Mass Spectrometry (ESI-MS)}

Mass spectrometric data were obtained by analysing samples on a Velos Pro mass spectrometer (Thermo Scientific) equipped with an ESI probe coupled to the RP-UHPLC system. Nitrogen was used as sheath gas and auxiliary gas. Data were collected over the $\mathrm{m} / \mathrm{z}$ range of 250-2000. Data-dependent $\mathrm{MS}^{\mathrm{n}}$ analysis was performed with a normalized collision energy of $35 \%$. The MS was run in two different modes: Full MS and MS/MS on selected ions. In full MS, the $\mathrm{MS}^{\mathrm{n}}$ fragmentation was performed on the most intense product ion in the $\mathrm{MS}^{\mathrm{n}-1}$ spectrum, with a dynamic exclusion for $5 \mathrm{~s}$, when the most intense product ion appeared twice within $5 \mathrm{~s}$. With MS/MS on selected ions, the MS will monitor the $\mathrm{MS}^{2}$ data of the parent ions specified in the MS/MS inclusion list. The system was tuned with theaflavin-3-gallate in negative ionization mode. Most settings were optimized via automatic tuning using "Tune Plus" of Xcalibur 2.07 (Thermo Scientific). The transfer tube temperature was $350{ }^{\circ} \mathrm{C}$, and the source voltage was $4.0 \mathrm{kV}$. Data acquisition and reprocessing were performed with Xcalibur 2.07.

\subsection{Results and Discussion}

\subsubsection{Enzymatic activity in tea leaves}

An enzyme extract from fresh tea leaves was prepared and tested for oxidative activities (Table 5.1). Incubations with epicatechin (EC) with and without $\mathrm{H}_{2} \mathrm{O}_{2}$ represented combined POD and PPO activities, and PPO activity alone, respectively. The combined POD plus PPO activity was approximately 4 times higher than PPO activity alone, indicating a 3 times higher POD than PPO activity, which is similar as reported previously. ${ }^{17}$

Table 5.1 Oxidative enzyme activity $\left({ }^{*} 10^{-6}\right)$ on specific substrates (AU/min). ${ }^{*}$

\begin{tabular}{cccccc}
\hline$\left(* 10^{-6}\right)$ & $\begin{array}{c}\text { PPO } \\
(E C)^{\dagger}\end{array}$ & $\begin{array}{c}\text { PPO + POD } \\
\left(E C+H_{2} \mathrm{O}_{2}\right)^{\dagger}\end{array}$ & $\begin{array}{c}\text { Laccase } \\
(A B T S)^{\dagger}\end{array}$ & $\begin{array}{c}\text { Cresolase } \\
(\text { Tyrosine })^{\ddagger}\end{array}$ & Blank \\
\hline $\begin{array}{c}\text { Enzyme } \\
\text { extract }\end{array}$ & $\begin{array}{c}66,600 \\
( \pm 5,100)\end{array}$ & $\begin{array}{c}204,000 \\
( \pm 8,400)\end{array}$ & $3,320( \pm 13)$ & $1,200( \pm 99)$ & $2,280( \pm 22)$ \\
Control $\$$ & $0( \pm 0.52)$ & $22.8( \pm 61.1)$ & $637( \pm 670)$ & $0( \pm 94)$ & $56.9( \pm 21)$ \\
\hline
\end{tabular}

${ }^{*}$ The values are the average of two measurements (plus standard deviation), ${ }^{\dagger}$ measured at $420 \mathrm{~nm}$, $\ddagger$ measured at $520 \mathrm{~nm}, \S$ the control sample is without enzyme.

PPO activity is rather ill-defined and can be subdivided into catecholase and cresolase activity. Sometimes laccase is considered a PPO as well, because it also uses oxygen as electron acceptor, thereby laccase would add up to a third activity in PPO. However, 
laccase forms radicals upon oxidation that can configure into quinones, whereas PPO directly forms $o$-quinones. ${ }^{21}$ To distinguish the different kinds of PPO activities in tea leaves, activity tests with ABTS and tyrosine were performed, to show laccase and cresolase activity, respectively (Table 5.1). Catecholase activity cannot be separately measured, because the substrates overlap with cresolase activity. Therefore, catecholase activity was determined by deduction. The laccase and cresolase activities measured were at least 10 times lower than the PPO activity measured on EC. Moreover, the standard deviations on the tyrosine and ABTS measurements were very high and did not differentiate the activities of both laccase and cresolase sufficiently from the change in absorbance in control samples without enzyme. Therefore, laccase and cresolase activity were considered negligible in the enzyme extract from tea leaves. Hence, the PPO activity in tea leaves is considered to mainly consist of catecholase activity, which is in line with previous research. ${ }^{22}$

\subsubsection{Hydroxylated theaflavin isomers}

To determine the possible hydroxylating activity of the two main oxidative enzymes in tea leaves, POD and PPO, a model system, using horseradish POD or mushroom tyrosinase (cresolase activity) was used. By using a model system, the time-consuming fractionation process of tea enzymes was circumvented. A fraction enriched in TF was incubated with the individual enzymes and analysed by UHPLC-MS. When incubated with tyrosinase, hardly any TF reacted, whereas after incubation with POD the TF concentration decreased considerably (data not shown). This is in accordance to previous research. ${ }^{23}$ This corroborates the idea that POD is a likely candidate for hydroxylation of tea phenolics. ${ }^{16}$

Although UHPLC-MS analysis showed a decrease in TF concentration (data not shown), peaks with an $m / z$ of $\mathrm{TF}+x \mathrm{OH}$ were not found. Therefore, samples were also analysed using the MS/MS on selected ions mode, an MS method to specifically screen for selected compounds in low abundance (Ch. 3). The resulting chromatograms with the $\mathrm{MS} / \mathrm{MS}$ on selected ions method for the $\mathrm{TF}+x \mathrm{OH}$ are shown in Figure 5.1. The MS information of the compounds, corresponding to the numbered peaks, are shown in Table 5.2.

The fragmentation pattern of $\mathrm{TF}$ is known and can be used to identify $\mathrm{TF}^{21,24}$ but the fragmentation patterns of $\mathrm{TF}+x \mathrm{OHs}$ are unknown. It was assumed that hydroxylation does not affect the fragmentation pattern. ${ }^{15,25,26}$ The fragmentation pattern of TF was used to derive the expected $\mathrm{MS}^{2}$ fragments of the $\mathrm{TF}+x \mathrm{OHs}$ (Sup. Table S5.1).

Table 5.3 summarizes the number of isomers detected per degree of hydroxylation, as well as the theoretical number of isomers. As expected, it was observed that the higher the degree of hydroxylation, the more isomers were formed, which led to lower concentrations 
per isomer. The TFs with extra hydroxyl groups eluted earlier than TF, which was expected due to their increased polarity (Fig. 5.1).

The fragmentation patterns of the $\mathrm{TF}+x \mathrm{OHs}$ were used to locate the position of the newly added hydroxyl groups. The TF fragments with $m / z 407,379$ and 425 all resulted from RDA cleavage in combination with losses of water and CO losses (Fig. 5.2). As a TF molecule has two free benzopyran moieties, these RDA fragments cannot give information on which of the catechin subunits was hydroxylated. The fragment with $\mathrm{m} / \mathrm{z} 241$ resulted from cleavage in the seven-membered ring of the benzotropolone moiety. Therefore, it can be used to identify on which catechin moiety the oxygen substitution occurred (Fig. 5.2). For instance, of the single hydroxylated TFs $(\mathrm{m} / \mathrm{z} 579)$ in Figure 5.1B compounds $\mathbf{2}$ and $\mathbf{9}$ showed the fragment of $m / z 241$. This indicated that the fragment ion, the EGC moiety, did not contain the extra hydroxyl group, but that the neutral loss, the EC moiety of TF, did (Fig. 5.2). Four other peaks (3,6-8) showed a fragment of $m / z 257$, which is $m / z 241$ plus 16 $\mathrm{Da}$. This indicated the hydroxyl group was located on the fragment ion, the EGC moiety of the TF.

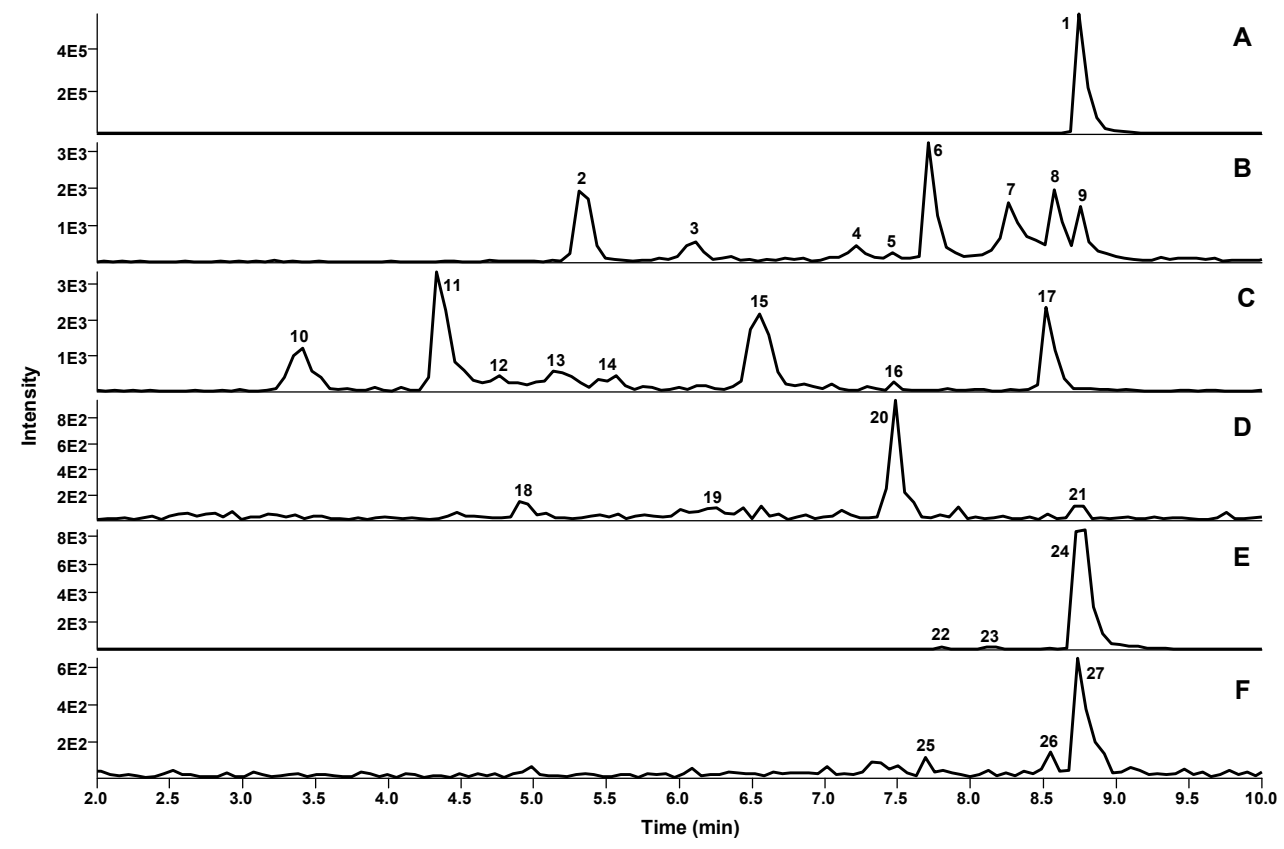

Figure 5.1 EICs of MS/MS on selected ions of a one hour incubation of TF with POD in the presence of $0.1 \mathrm{mM} \mathrm{H}_{2} \mathrm{O}_{2}$. The following ions were screened for per measurement: A) $m / z 563$, B) $m / z 579$, C) $m / z 595$, D) $m / z 611$, E) $m / z 627$, and F) $m / z$ 643. Fragments extracted in the EICs are according to the values in Supplemental Table S5.1. The compounds corresponding to the numbered peaks can be found in Table 5.2. 
POD is responsible for hydroxylation during black tea processing

Table 5.2 TF $+x \mathrm{OHs}$ found by RP-UHPLC-MS ${ }^{\mathrm{n}}$ from the TF incubation with POD and $0.1 \mathrm{mM}$ $\mathrm{H}_{2} \mathrm{O}_{2}$, tentatively annotated based on parent ion related $\mathrm{MS}^{2}$ fragments.

\begin{tabular}{|c|c|c|c|c|}
\hline $\begin{array}{l}\text { Peak } \\
\text { no. }\end{array}$ & $\begin{array}{l}\text { Retention } \\
\text { time (min) }\end{array}$ & $\underset{(m / z)}{[\mathrm{M}-\mathrm{H}]^{-}}$ & $\begin{array}{l}\text { Parent related } \mathrm{MS}^{2} \text { fragments } \\
\qquad(\mathrm{m} / \mathrm{z})\end{array}$ & $\begin{array}{l}\text { Tentative } \\
\text { annotation }\end{array}$ \\
\hline 1 & 8.75 & 563 & $407,379,241,425$ & $\mathrm{TF}$ \\
\hline 2 & 5.31 & 579 & $395,241,257,441,423,379$ & $\mathrm{TF}+\mathrm{OH}$ \\
\hline 3 & 6.11 & 579 & $395,441,257,241,423$ & $\mathrm{TF}+\mathrm{OH}$ \\
\hline 4 & 7.22 & 579 & $441,423,395,379$ & $\mathrm{TF}+\mathrm{OH}$ \\
\hline 5 & 7.46 & 579 & $441,395,379$ & $\mathrm{TF}+\mathrm{OH}$ \\
\hline 6 & 7.71 & 579 & $425,423,257,395,441$ & $\mathrm{TF}+\mathrm{OH}$ \\
\hline 7 & 8.26 & 579 & $441,423,395,379,257$ & $\mathrm{TF}+\mathrm{OH}$ \\
\hline 8 & 8.57 & 579 & $441,423,395,379,257$ & $\mathrm{TF}+\mathrm{OH}$ \\
\hline 9 & 8.76 & 579 & $425,441,407,423,395,241,257$ & $\mathrm{TF}+\mathrm{OH}$ \\
\hline 10 & 3.41 & 595 & $457,439,395,411,425,241,257$ & $\mathrm{TF}+2 \mathrm{OH}$ \\
\hline 11 & 4.34 & 595 & $439,457,407,395,425$ & $\mathrm{TF}+2 \mathrm{OH}$ \\
\hline 12 & 4.77 & 595 & $439,457,407,395,241,257$ & $\mathrm{TF}+2 \mathrm{OH}$ \\
\hline 13 & 5.14 & 595 & $457,439,395,407,425$ & $\mathrm{TF}+2 \mathrm{OH}$ \\
\hline 14 & 5.57 & 595 & $457,407,395$ & $\mathrm{TF}+2 \mathrm{OH}$ \\
\hline 15 & 6.55 & 595 & $457,439,395,257$ & $\mathrm{TF}+2 \mathrm{OH}$ \\
\hline 16 & 7.47 & 595 & $457,407,257,241,425$ & $\mathrm{TF}+2 \mathrm{OH}$ \\
\hline 17 & 8.52 & 595 & $457,439,411,395$ & $\mathrm{TF}+2 \mathrm{OH}$ \\
\hline 18 & 4.90 & 611 & $411,289,473,257$ & $\mathrm{TF}+3 \mathrm{OH}$ \\
\hline 19 & 6.25 & 611 & $473,425,395$ & $\mathrm{TF}+3 \mathrm{OH}$ \\
\hline 20 & 7.48 & 611 & $473,425,379,455$ & $\mathrm{TF}+3 \mathrm{OH}$ \\
\hline 21 & 8.78 & 611 & 473,439 & $\mathrm{TF}+3 \mathrm{OH}$ \\
\hline 22 & 7.80 & 627 & 489 & $\mathrm{TF}+4 \mathrm{OH}$ \\
\hline 23 & 8.11 & 627 & 489 & $\mathrm{TF}+4 \mathrm{OH}$ \\
\hline 24 & 8.79 & 627 & $489,471,441,423$ & $\mathrm{TF}+4 \mathrm{OH}$ \\
\hline 25 & 7.69 & 643 & $505,487,459,489$ & $\mathrm{TF}+5 \mathrm{OH}$ \\
\hline 26 & 8.55 & 643 & 505,459 & $\mathrm{TF}+5 \mathrm{OH}$ \\
\hline 27 & 8.74 & 643 & $505,487,441$ & $\mathrm{TF}+5 \mathrm{OH}$ \\
\hline
\end{tabular}




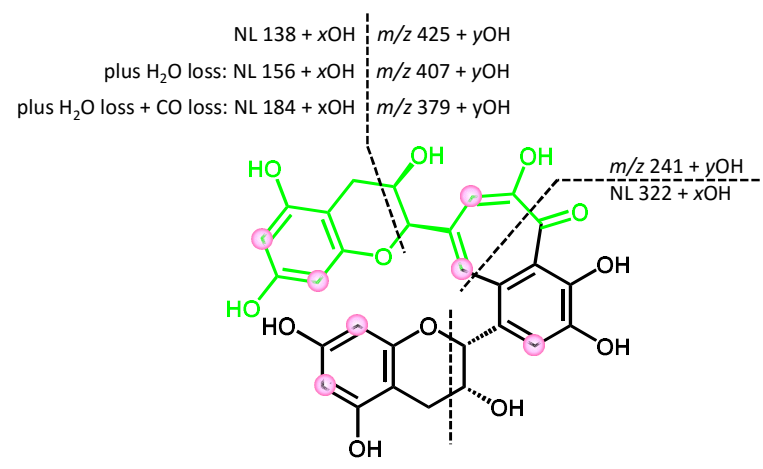

Figure 5.2 Structural representation of theaflavin, with the initial EC moiety in black, and the EGC moiety in green. Positions available for hydroxylation are indicated with a pink circle. The tentative $\mathrm{MS}^{2}$ fragmentation is indicated.

\subsubsection{Degree of hydroxylation in time}

The hydroxylation for different tea phenolics was monitored in time (Fig. 5.3). For all compounds a fast decrease in the starting compound was visible in the first minutes, after which the decrease levelled off (Fig. 5.3A). For TF, TNQ and T3D, it was clearly visible that the compounds with one extra hydroxyl group increased in time. For TF and TNQ also an increase in the compounds with two extra hydroxyl groups was observed (Fig. 5.3B-D). The compounds with three, four and five extra hydroxyl groups showed only a slight increase in time, as was expected from Figure 5.1, where they could hardly be detected as well. Hence, further discussion will only consider compounds with up to two extra hydroxyl groups. DhCs $\beta_{\mathrm{AB}} / \varepsilon_{\mathrm{AB}}$ and $\mathrm{DhC} \gamma_{\mathrm{AB}} / \delta_{\mathrm{AB}}$ will also not be discussed further, because little hydroxylation was observed (data not shown). Possibly, a reaction other than hydroxylation occurred with these compounds, because they decreased during incubation with POD and $\mathrm{H}_{2} \mathrm{O}_{2}$. Another explanation might be that hydroxylation gave too many isomers, all below the limit of detection.

TF (Fig. 5.3B) and TNQ (Fig. 5.3C) showed the expected pattern for the compounds with one and two extra hydroxyl groups, respectively. The compounds with one extra hydroxyl group increased in time, then started to decrease after around $45 \mathrm{~min}$, whereas the compounds with two extra hydroxyl groups kept increasing. Clearly, the compounds with one extra hydroxyl group are precursors for the compounds with two extra hydroxyl groups. For T3D (Fig. 5.3D) the T3Ds with one extra hydroxyl group increased in time, until a plateau level, whereas the T3Ds with two extra hydroxyl groups remained at a very low concentration. 
POD is responsible for hydroxylation during black tea processing

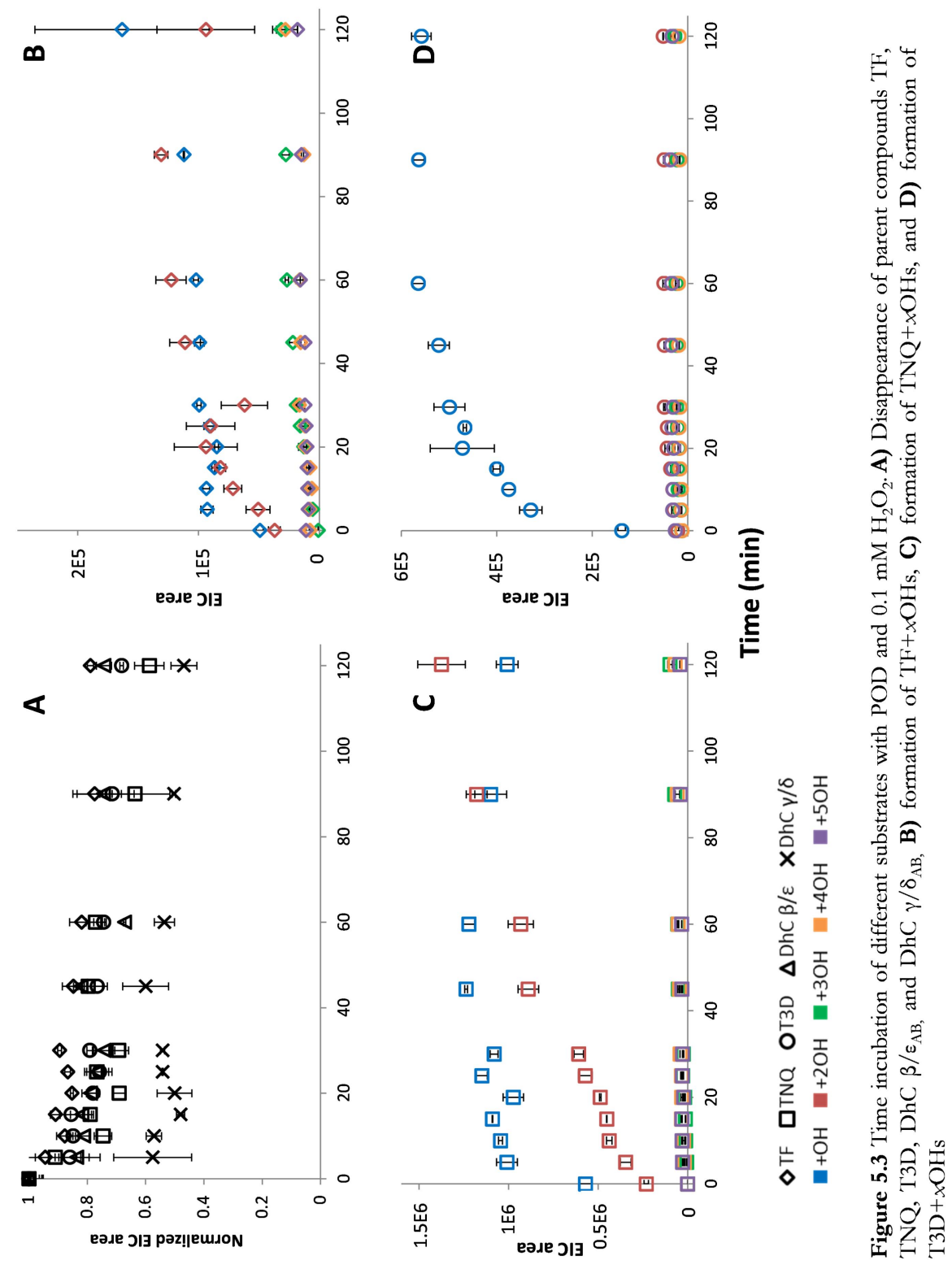


Table 5.3. The theoretical number of isomers and the number of isomers found experimentally for the different degrees of hydroxylation of theaflavins $(\mathrm{TF}+\chi \mathrm{OH})$. Peak numbers refer to Figure 5.1 and Table 5.2.

\begin{tabular}{lcccc}
\hline Identity & {$[\mathbf{M}-\mathbf{H}] \mathbf{m} / \mathbf{z}$} & Theoretical number of isomers & Number of isomers found & Peak numbers \\
\hline $\mathbf{T F}$ & 563 & 1 & 1 & 1 \\
$\mathbf{T F}+\mathbf{O H}$ & 579 & 7 & 8 & $2-9$ \\
\hline $\mathbf{T F}+\mathbf{2 O H}$ & 595 & 210 & 8 & $10-17$ \\
$\mathbf{T F}+\mathbf{3 O H}$ & 611 & 840 & 4 & $18-21$ \\
\hline $\mathbf{T F}+\mathbf{4 O H}$ & 627 & 2,520 & 3 & $22-24$ \\
\hline $\mathbf{T F}+\mathbf{5 O H}$ & 643 & 5,040 & 3 & $25-27$ \\
\hline
\end{tabular}

\subsubsection{Influence of $\mathrm{H}_{2} \mathrm{O}_{2}$ concentration on hydroxylation by POD}

The concentration of $\mathrm{H}_{2} \mathrm{O}_{2}$ is also a factor that could influence the hydroxylation of the compounds. In tea leaves it has been shown that PPO activity on tea catechins resulted in formation of $\mathrm{H}_{2} \mathrm{O}_{2} \cdot{ }^{19,27}$ In steady state fermentation, the $\mathrm{H}_{2} \mathrm{O}_{2}$ concentration was shown to be $0.07-0.09 \mathrm{mM},{ }^{19}$ a concentration similar to that used so far during the experiments in this research $\left(0.1 \mathrm{mM} \mathrm{H}_{2} \mathrm{O}_{2}\right)$.

Figure 5.4 shows the results of a one hour incubation of TF with POD at $\mathrm{H}_{2} \mathrm{O}_{2}$ concentrations varying around $0.1 \mathrm{mM}$. On the one hand, the concentration of TFs with one extra hydroxyl group slightly decreased with decreasing $\mathrm{H}_{2} \mathrm{O}_{2}$ concentration. On the other hand, a clear increase in TFs with two extra hydroxyl groups was observed with decreasing $\mathrm{H}_{2} \mathrm{O}_{2}$ concentration. This is in accordance with each other, as $\mathrm{TF}+2 \mathrm{OHs}$ are formed from $\mathrm{TF}+\mathrm{OHs}$. At most concentrations of $\mathrm{H}_{2} \mathrm{O}_{2}$, only $25 \%$ of the initial $\mathrm{TF}$ was extra hydroxylated.

At lower $\mathrm{H}_{2} \mathrm{O}_{2}$ concentrations, the hydroxylation reaction appeared to occur more extensively than at higher concentrations. This might be due to inhibition of POD by $\mathrm{H}_{2} \mathrm{O}_{2}$, which has been described before. ${ }^{28}$ The concentration of $\mathrm{H}_{2} \mathrm{O}_{2}$ occurring in tea leaves, i.e. 0.07-0.09 $\mathrm{mM},{ }^{19}$ appears to be the optimal for hydroxylation during tea oxidation.

\subsubsection{Hydroxylated theaflavins in black tea}

An MS method of MS/MS on selected ions was used to specifically screen for $\mathrm{TF}+x \mathrm{OHs}$ in black tea. Figure $\mathbf{5 . 5}$ clearly shows the presence of $\mathrm{TF}+x \mathrm{OH}$ in the black tea, with TFs containing up to five extra hydroxyl groups. 
Considering the intensities of the compounds, the TF+2OHs (Fig. 5.5D) seemed to have the highest intensity amongst the different degrees of hydroxylation, however, still at a 1,000x lower intensity than the most abundant phenolics present in black tea, i.e. the nonoxidized catechins and theaflavins. The $\mathrm{TF}+2 \mathrm{OHs}$ were followed by the $\mathrm{TF}+3 \mathrm{OHs}$ (Fig. 5.5E) at a 10x lower intensity than the $\mathrm{TF}+2 \mathrm{OHs}$. Subsequently, the TF+OHs (Fig. 5.5C) and $\mathrm{TF}+4 \mathrm{OHs}$ (Fig. 5.5F) were present at a 100x lower intensity than the $\mathrm{TF}+2 \mathrm{OHs}$. Finally the $\mathrm{TF}+5 \mathrm{OHs}$ (Fig. 5.5G) were present at a 1,000x lower intensity than the $\mathrm{TF}+2 \mathrm{OHs}$. This intensity of the $\mathrm{TF}+5 \mathrm{OHs}$ is a factor $10^{6}$ lower than the intensities of the most abundant black tea phenolics in the sample. This showed that hydroxylated compounds can be identified by their $\mathrm{MS}^{2}$ data with the MS/MS on selected ions method, even at intensities $10^{6}$ times lower than that of the most abundant phenolics.

The presence of TNQs, T3Ds, and DhCs with extra hydroxyl groups was tested as well with the MS/MS on selected ions method. Traces of these compounds with extra hydroxyl groups were found in black tea (no further data shown).
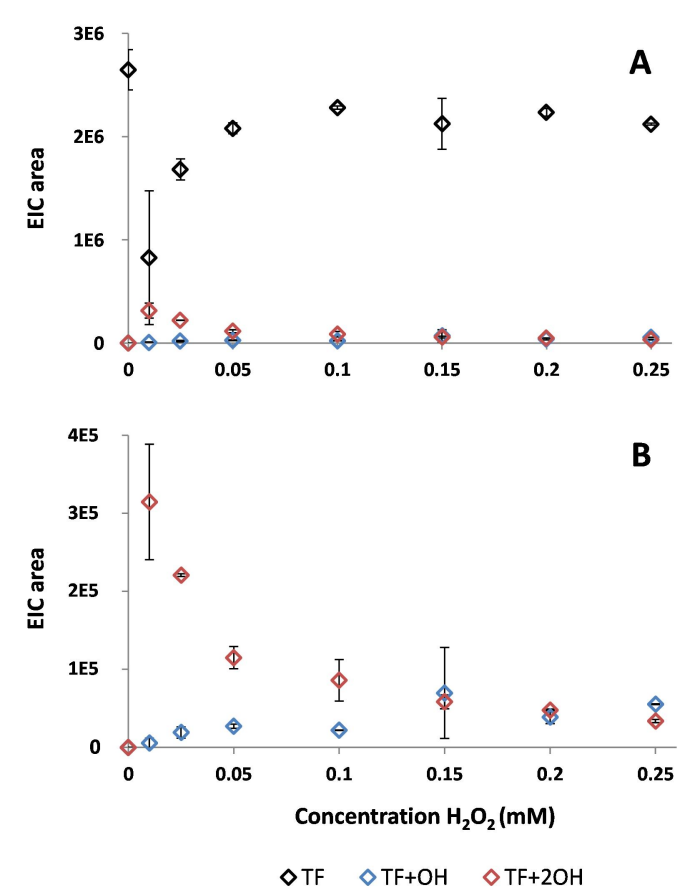

Figure 5.4 A) Presence of $\mathrm{TF}$ and $\mathrm{TF}+x \mathrm{OHs}$, after one hour incubation of TF with POD at various $\mathrm{H}_{2} \mathrm{O}_{2}$ concentrations. B) zoomed in version of the $\mathrm{TF}+\chi \mathrm{OHs}$ in the incubation. Occurrence of $\mathrm{TF}+x \mathrm{OHs}$ is based on EICs of $\mathrm{MS}^{2}$ signal according to the values in Supplemental Table S5.1. The values are the average of two measurements. 


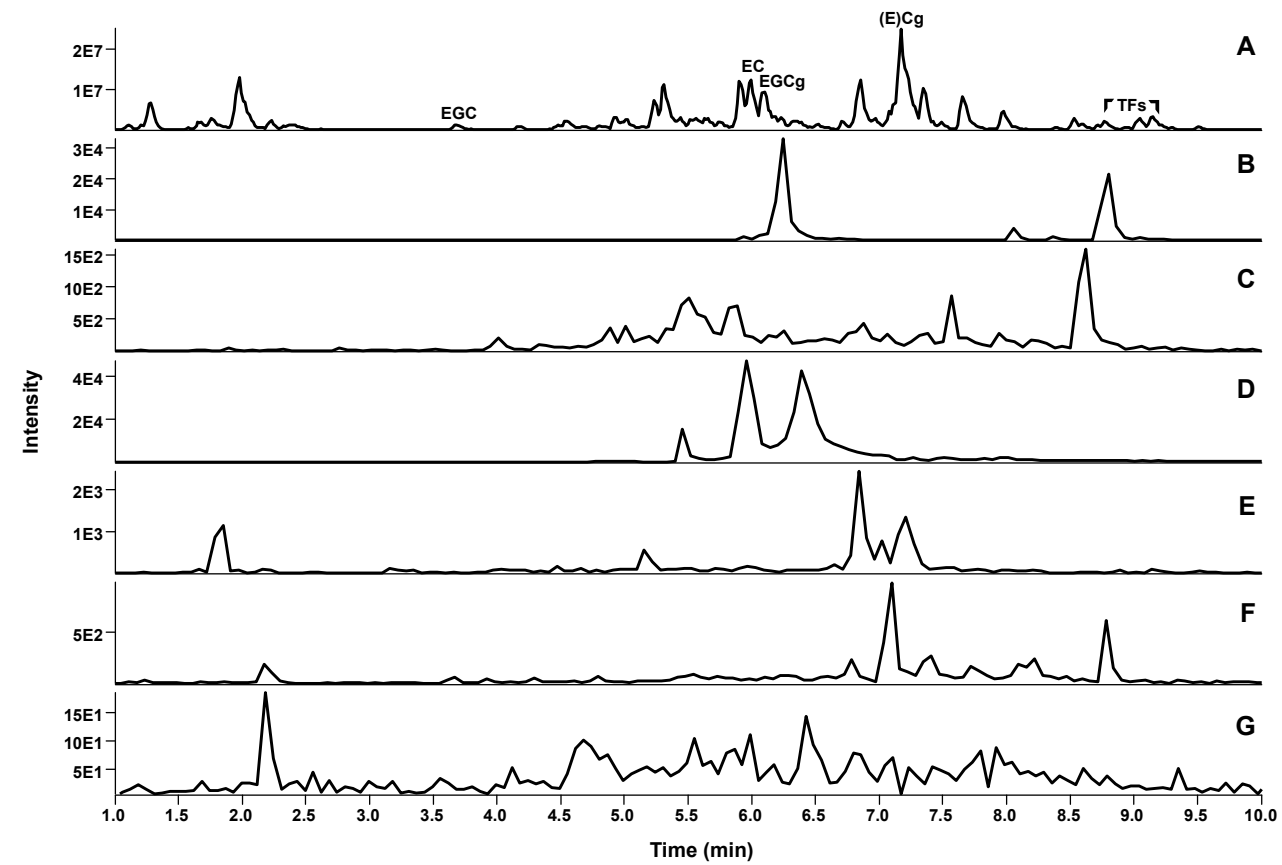

Figure 5.5 RP-UHPLC-MS chromatograms of a Chinese black Yunnan tea. A) Full MS, B) EIC of MS/MS on selected ion $m / z 563(\mathrm{TF}), \mathbf{C})$ EIC of MS/MS on selected ion $m / z .579(\mathrm{TF}+\mathrm{OH}), \mathbf{D})$ EIC of MS/MS on selected ion $\mathrm{m} / z 595(\mathrm{TF}+2 \mathrm{OH}), \mathbf{E})$ EIC of MS/MS on selected ion $\mathrm{m} / z_{6} 611$ $(\mathrm{TF}+3 \mathrm{OH}), \mathbf{F})$ EIC of MS/MS on selected ion $m / z 627(\mathrm{TF}+4 \mathrm{OH})$, and $\mathbf{G}) \mathrm{EIC}$ of MS/MS on selected ion $\mathrm{m} / z, 643(\mathrm{TF}+5 \mathrm{OH})$. Fragments extracted in the EICs are according to the values in Supplemental Table S5.1.

\subsubsection{Enzymatic browning in tea leaves}

This study demonstrated the role of POD in the hydroxylation of tea phenolics and therewith established an important missing link to the understanding of enzymatic browning in tea leaves. Figure $\mathbf{5 . 6}$ schematically summarizes the currently known (enzymatic) oxidation reactions of the 'oxidative cascade hypothesis', including various oligomerization steps, intramolecular rearrangements and hydroxylation.

PPO is mainly responsible for the first oligomerization of the catechins. This involves a basic oligomerization step resulting in either the dimers TF, TS, TC or DhC $\beta / \varepsilon_{\mathrm{AB}}{ }^{l}$ (Ch. 4). Simultaneous with these oxidation reactions, limited amounts of $\mathrm{H}_{2} \mathrm{O}_{2}$ are formed by PPO.$^{19,27}$ For the subsequent oxidation steps, both PPO and POD can be responsible. The subsequent oxidation steps can be either another oligomerization or an intramolecular rearrangement. For the oligomerization, a catechin can be added via another basic 
oligomerization step, as in the formation of $\mathrm{TFsEC}^{29}(\mathbf{C h} \mathbf{3}$ ), where a third catechin is added via a TS-configuration to a TF. A catechin can also be added via a tripartite oligomerization type, like for T3D, where a new catechin unit is superimposed on an existing interflavanic configuration. ${ }^{11}$ Oxidation of the oligomers can also result in intramolecular rearrangements. For instance, TNQ is formed ${ }^{2}$ from rearrangements when $\mathrm{TF}$ is oxidized. Also, when TNQ is oxidized, it can rearrange into TCou ${ }^{13}$. As stated, POD can also be responsible for oxidation reactions leading to oligomerization, from the second oxidation step onwards. Nevertheless, in Figure 5.6, mainly lines representing PPO activity are drawn, as PPO seems to be a more likely candidate than POD to perform an oligomerization reaction, due to the limited availability of $\mathrm{H}_{2} \mathrm{O}_{2}$, especially at the onset of tea leaf oxidation.

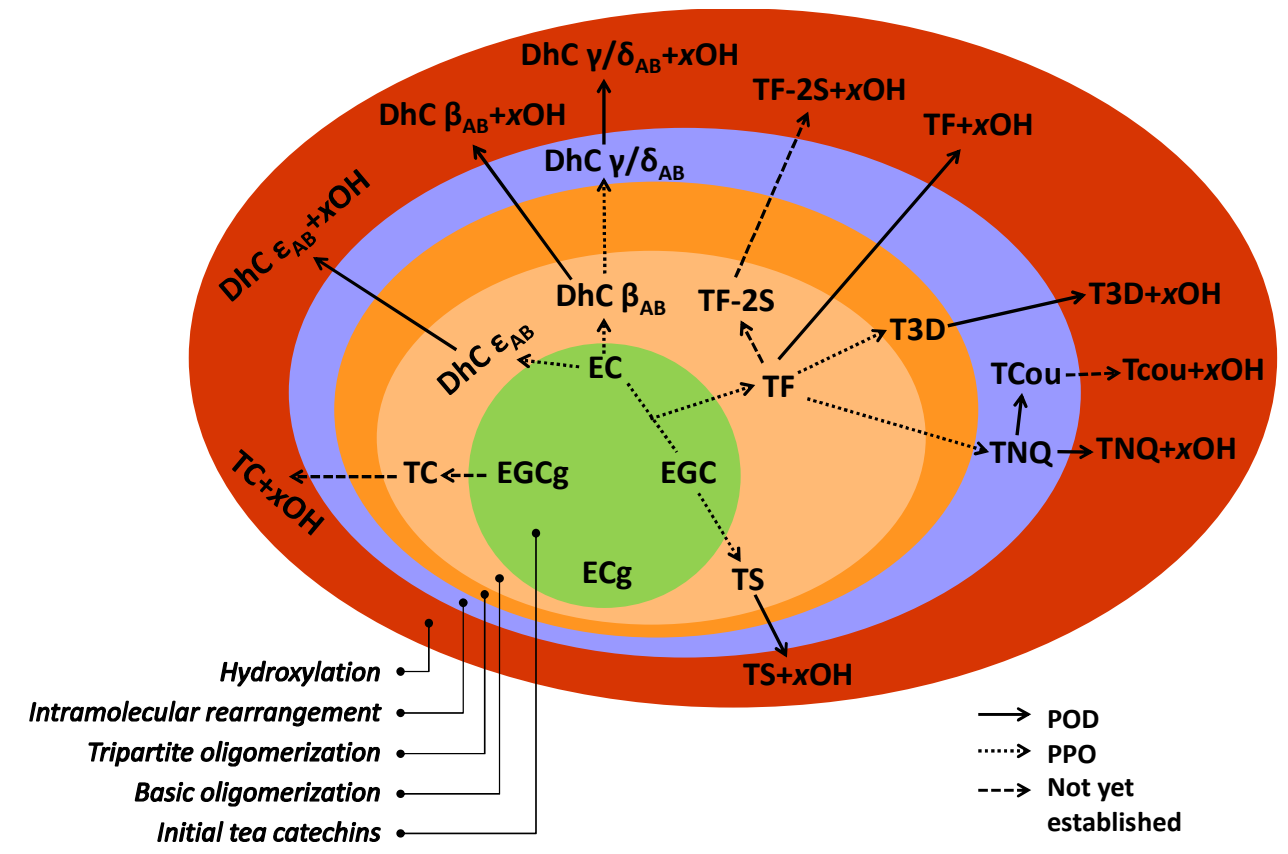

Figure 5.6 Schematic overview of the oxidation reactions occurring in tea leaves, together with the enzymes responsible.

It seems plausible that POD is the enzyme in tea leaves, which is responsible for the hydroxylation of the phenolics. Hydroxylating activity of POD has been reported before, albeit only sparsely. ${ }^{30}$ The mechanism by which POD hydroxylates is still unknown, but it might resemble the hydroxylating activity of peroxygenases, which have a heme group and peroxide as oxidizing agent in common with POD. Peroxygenases have been shown to 
hydroxylate by oxygen transfer from $\mathrm{H}_{2} \mathrm{O}_{2}$ onto the aromatic ring, after which an intermediate epoxide is formed that can spontaneously rearrange into a hydroxyl. ${ }^{31}$

All oligomeric compounds formed are likely susceptible to hydroxylation by POD, and the hydroxylation can occur simultaneously with the other oxidation reactions, as soon as $\mathrm{H}_{2} \mathrm{O}_{2}$ is formed. Even though in Figure 5.6 the hydroxylation is depicted as the final layer, hydroxylation is not necessarily the final oxidation step. After the first oligomerization step, all three oxidation reactions, oligomerization, intramolecular rearrangement and hydroxylation, can occur in random order.

\subsection{References}

1. Kuhnert, N.; Drynan, J. W.; Obuchowicz, J.; Clifford, M. N.; Witt, M. Mass spectrometric characterization of black tea thearubigins leading to an oxidative cascade hypothesis for thearubigin formation. Rapid Communications in Mass Spectrometry 2010, 24, 3387-3404.

2. Drynan, J. W.; Clifford, M. N.; Obuchowicz, J.; Kuhnert, N. The chemistry of low molecular weight black tea polyphenols. Natural Product Reports 2010, 27, 417-462.

3. Takino, Y.; Ferretti, A.; Flanagan, V.; Gianturco, M.; Vogel, M. The structure of theaflavin, A polyphenol of black tea. Tetrahedron Letters 1965, 6, 4019-4025.

4. Takino, Y.; Imagawa, H.; Harikawa, H.; Tanaka, A. Studies on the mechanism of the oxidation of the catechins. Part III, Formation of a reddish-orange pigment, its spectral relationship to some benzotropolone derivatives. Agriculture, Biology and Chemistry 1964, 28, 64-71.

5. Matsuo, Y.; Tanaka, T.; Kouno, I. A new mechanism for oxidation of epigallocatechin and production of benzotropolone pigments. Tetrahedron 2006, 62, 4774-4783.

6. Tanaka, T.; Mine, C.; Watarumi, S.; Fujioka, T.; Mihashi, K.; Zhang, Y. J.; Kouno, I. Accumulation of epigallocatechin quinone dimers during tea fermentation and formation of theasinensins. Journal of Natural Products 2002, 65, 1582-1587.

7. Tanaka, T.; Watarumi, S.; Matsuo, Y.; Kamei, M.; Kouno, I. Production of theasinensins A and D, epigallocatechin gallate dimers of black tea, by oxidation-reduction dismutation of dehydrotheasinensin A. Tetrahedron 2003, 59, 7939-7947.

8. Matsuo, Y.; Li, Y.; Watarumi, S.; Tanaka, T.; Kouno, I. Production and degradation mechanism of theacitrin $\mathrm{C}$, a black tea pigment derived from epigallocatechin-3-O-gallate via a bicyclo[3.2.1]octane-type intermediate. Tetrahedron 2011, 67, 2051-2059.

9. Davis, A. L.; Lewis, J. R.; Cai, Y.; Powell, C.; Davis, A. P.; Wilkins, J. P. G.; Pudney, P.; Clifford, M. N. A polyphenolic pigment from black tea. Phytochemistry 1997, 46, 1397-1402.

10. Guyot, S.; Vercauteren, J.; Cheynier, V. Structural determination of colourless and yellow dimers resulting from (+)-catechin coupling catalysed by grape polyphenoloxidase. Phytochemistry 1996, 42, 1279-1288.

11. Li, Y.; Shibahara, A.; Matsuo, Y.; Tanaka, T.; Kouno, I. Reaction of the black tea pigment theaflavin during enzymatic oxidation of tea catechins. Journal of Natural Products 2010, 73, 33-39.

12. Tanaka, T.; Betsumiya, Y.; Mine, C.; Kouno, I. Theanaphthoquinone, a novel pigment oxidatively derived from theaflavin during tea-fermentation. Chemical Communications 2000, 1365-1366.

13. Kusano, R.; Matsuo, Y.; Saito, Y.; Tanaka, T. Oxidation mechanism of black tea pigment theaflavin by peroxidase. Tetrahedron Letters 2015, 56, 5099-5102. 
14. Kuhnert, N.; Clifford, M. N.; Müller, A. Oxidative cascade reactions yielding polyhydroxy-theaflavins and theacitrins in the formation of black tea thearubigins: Evidence by tandem LC-MS. Food and Function 2010, 1, 180-199.

15. Yassin, G. H.; Koek, J. H.; Jayaraman, S.; Kuhnert, N. Identification of novel homologous series of polyhydroxylated theasinensins and theanaphthoquinones in the SII fraction of black tea thearubigins using ESI/HPLC tandem mass spectrometry. Journal of Agricultural and Food Chemistry 2014, 62, 9848-9859.

16. Stodt, U. W.; Blauth, N.; Niemann, S.; Stark, J.; Pawar, V.; Jayaraman, S.; Koek, J.; Engelhardt, U. H. Investigation of processes in black tea manufacture through model fermentation (oxidation) experiments. Journal of Agricultural and Food Chemistry 2014, 62, 7854-7861.

17. Mahanta, P. K.; Boruah, S. K.; Boruah, H. K.; Kalita, J. N. Changes of polyphenol oxidase and peroxidase activities pigment composition of some manufactured black teas (Camellia, sinensis L.). Journal of Agricultural and Food Chemistry 1993, 41, 272-276.

18. Samanta, T.; Cheeni, V.; Das, S.; Roy, A. B.; Ghosh, B. C.; Mitra, A. Assessing biochemical changes during standardization of fermentation time and temperature for manufacturing quality black tea. Journal of Food Science and Technology 2013, 1-7.

19. Subramanian, N.; Venkatesh, P.; Ganguli, S.; Sinkar, V. P. Role of polyphenol oxidase and peroxidase in the generation of black tea theaflavins. Journal of Agricultural and Food Chemistry 1999, 47, 2571-2578.

20. Bohin, M. C.; Vincken, J. P.; Van Der Hijden, H. T. W. M.; Gruppen, H. Efficacy of food proteins as carriers for flavonoids. Journal of Agricultural and Food Chemistry 2012, 60, 4136-4143.

21. Verloop, A. J. W.; Gruppen, H.; Bisschop, R.; Vincken, J. P. Altering the phenolics profile of a green tea leaves extract using exogenous oxidases. Food Chemistry 2016, 196, 1197-1206.

22. Halder, J.; Tamuli, P.; Bhaduri, A. N. Isolation and characterization of polyphenol oxidase from Indian tea leaf (Camellia sinensis). Journal of Nutritional Biochemistry 1998, 9, 75-80.

23. Stagg, G. V. Chemical changes occurring during the storage of black tea. Journal of the Science of Food and Agriculture 1974, 25, 1015-1034.

24. Dou, J.; Lee, V. S. Y.; Tzen, J. T. C.; Lee, M. R. Identification and comparison of phenolic compounds in the preparation of oolong tea manufactured by semifermentation and drying processes. Journal of Agricultural and Food Chemistry 2007, 55, 7462-7468.

25. Cuyckens, F.; Claeys, M. Mass spectrometry in the structural analysis of flavonoids. Journal of Mass Spectrometry 2004, 39, 1-15.

26. Cren-Olivé, C.; Déprez, S.; Lebrun, S.; Coddeville, B.; Rolando, C. Characterization of methylation site of monomethylflavan-3-ols by liquid chromatography/electrospray ionization tandem mass spectrometry. Rapid Communications in Mass Spectrometry 2000, 14, 2312-2319.

27. Jiang, Y.; Miles, P. W. Generation of $\mathrm{H}_{2} \mathrm{O}_{2}$ during enzymic oxidation of catechin. Phytochemistry 1993, 33, 29-34.

28. Jouili, H.; Bouazizi, H.; Rossignol, M.; Borderies, G.; Jamet, E.; El Ferjani, E. Partial purification and characterization of a copper-induced anionic peroxidase of sunflower roots. Plant Physiology and Biochemistry 2008, 46, 760-767.

29. Yassin, G. H.; Koek, J. H.; Kuhnert, N. Identification of trimeric and tetrameric flavan-3-ol derivatives in the SII black tea thearubigin fraction of black tea using ESI-tandem and MALDI-TOF mass spectrometry. Food Research International 2014, 63, 317-327.

30. Ullrich, R.; Hofrichter, M. Enzymatic hydroxylation of aromatic compounds. Cellular and Molecular Life Sciences 2007, 64, 271-293.

31. Kluge, M.; Ullrich, R.; Dolge, C.; Scheibner, K.; Hofrichter, M. Hydroxylation of naphthalene by aromatic peroxygenase from Agrocybe aegerita proceeds via oxygen transfer from $\mathrm{H}_{2} \mathrm{O}_{2}$ and intermediary epoxidation. Applied Microbiology and Biotechnology 2009, 81, 1071-1076. 


\subsection{Supplementary data}

Table S5.1 Selected parent ions and fragments to screen for in EIC.

\begin{tabular}{|c|c|c|}
\hline $\begin{array}{l}\text { Selected } \\
\text { parent } \\
\text { ion }(m / z) \\
\end{array}$ & Identity & Fragments screened in EIC $(m / z)$ \\
\hline 563 & $\mathrm{TF}$ & $379,407,425,241$ \\
\hline 579 & $\mathrm{TF}+\mathrm{OH}$ & $379,407,425,241,395,423,441,257$ \\
\hline 595 & $\mathrm{TF}+2 \mathrm{OH}$ & $379,407,425,241,395,423,441,257,411,439,457,273$ \\
\hline 611 & $\mathrm{TF}+3 \mathrm{OH}$ & $\begin{array}{l}379,407,425,241,395,423,441,257,411,439,457,273,427,455, \\
473,289\end{array}$ \\
\hline 627 & $\mathrm{TF}+4 \mathrm{OH}$ & $\begin{array}{l}379,407,425,241,395,423,441,257,411,439,457,273,427,455 \\
473,289,443,471,489,305\end{array}$ \\
\hline 643 & $\mathrm{TF}+5 \mathrm{OH}$ & $\begin{array}{l}379,407,425,241,395,423,441,257,411,439,457,273,427,455, \\
473,289,443,471,489,305,459,487,505,321\end{array}$ \\
\hline 851 & T3D & $617,713,815$ \\
\hline 867 & $\mathrm{~T} 3 \mathrm{D}+\mathrm{OH}$ & $617,713,815,633,729,831$ \\
\hline 883 & $\mathrm{~T} 3 \mathrm{D}+2 \mathrm{OH}$ & $617,713,815,633,729,831,649,745,847$ \\
\hline 899 & $\mathrm{~T} 3 \mathrm{D}+3 \mathrm{OH}$ & $617,713,815,633,729,831,649,745,847,665,761,863$ \\
\hline 915 & $\mathrm{~T} 3 \mathrm{D}+4 \mathrm{OH}$ & $617,713,815,633,729,831,649,745,847,665,761,863,681,777,879$ \\
\hline 931 & $\mathrm{~T} 3 \mathrm{D}+5 \mathrm{OH}$ & $\begin{array}{l}617,713,815,633,729,831,649,745,847,665,761,863,681,777, \\
879,697,793,895\end{array}$ \\
\hline 533 & TNQ & $505,471,349,305$ \\
\hline 549 & $\mathrm{TNQ}+\mathrm{OH}$ & $505,471,349,305,521,487,365,321$ \\
\hline 565 & $\mathrm{TNQ}+2 \mathrm{OH}$ & $505,471,349,305,521,487,365,321,537,503,381,337$ \\
\hline 581 & $\mathrm{TNQ}+3 \mathrm{OH}$ & $\begin{array}{l}505,471,349,305,521,487,365,321,537,503,381,337,553,519, \\
397,353\end{array}$ \\
\hline 597 & $\mathrm{TNQ}+4 \mathrm{OH}$ & $\begin{array}{l}505,471,349,305,521,487,365,321,537,503,381,337,553,519, \\
397,353,569,535,413,369\end{array}$ \\
\hline 613 & $\mathrm{TNQ}+5 \mathrm{OH}$ & $\begin{array}{l}505,471,349,305,521,487,365,321,537,503,381,337,553,519, \\
397,353,569,535,413,369,585,551,429,385\end{array}$ \\
\hline 575 & $\mathrm{DhC} \gamma / \delta_{\mathrm{AB}}$ & $394,493,411,515$ \\
\hline 591 & $\mathrm{DhC} \gamma / \delta_{\mathrm{AB}}+\mathrm{OH}$ & $394,493,411,515,410,509,427,531$ \\
\hline 607 & $\mathrm{DhC} \gamma / \delta_{\mathrm{AB}}+2 \mathrm{OH}$ & $394,493,411,515,410,509,427,531,426,525,443,547$ \\
\hline 623 & $\mathrm{DhC} \gamma / \delta_{\mathrm{AB}}+3 \mathrm{OH}$ & $\begin{array}{l}394,493,411,515,410,509,427,531,426,525,443,547,442,541, \\
459,563\end{array}$ \\
\hline 639 & $\mathrm{DhC} \gamma / \delta_{\mathrm{AB}}+4 \mathrm{OH}$ & $\begin{array}{l}394,493,411,515,410,509,427,531,426,525,443,547,442,541, \\
459,563,458,557,475,579\end{array}$ \\
\hline 655 & $\mathrm{DhC} \gamma / \delta_{\mathrm{AB}}+5 \mathrm{OH}$ & $\begin{array}{l}394,493,411,515,410,509,427,531,426,525,443,547,442,541, \\
459,563,458,557,475,579,474,573,491,595\end{array}$ \\
\hline 577 & $\mathrm{DhC} \beta / \varepsilon_{\mathrm{AB}}$ & $439,393,533,289$ \\
\hline 593 & $\mathrm{DhC} \beta / \varepsilon_{\mathrm{AB}}+\mathrm{OH}$ & $439,393,533,289,455,409,549,305$ \\
\hline 609 & $\mathrm{DhC} \beta / \varepsilon_{\mathrm{AB}}+2 \mathrm{OH}$ & $439,393,533,289,455,409,549,305,471,425,565,321$ \\
\hline 625 & $\mathrm{DhC} \beta / \varepsilon_{\mathrm{AB}}+3 \mathrm{OH}$ & $\begin{array}{l}439,393,533,289,455,409,549,305,471,425,565,321,487,441, \\
581,337\end{array}$ \\
\hline 641 & $\mathrm{DhC} \beta / \varepsilon_{\mathrm{AB}}+4 \mathrm{OH}$ & $\begin{array}{l}439,393,533,289,455,409,549,305,471,425,565,321,487,441, \\
581,337,503,457,597,353\end{array}$ \\
\hline 657 & $\mathrm{DhC} \beta / \varepsilon_{\mathrm{AB}}+5 \mathrm{OH}$ & $\begin{array}{l}439,393,533,289,455,409,549,305,471,425,565,321,487,441, \\
581,337,503,457,597,353,519,473,613,369\end{array}$ \\
\hline
\end{tabular}


Chapter 6

General discussion 
With the objective to make the oxidation of black tea a more controlled process, the research described in this thesis was aimed at (i) obtaining knowledge of the oxidation reactions occurring during the manufacturing of black tea, and at (ii) developing rapid screening methods for the annotation of oxidation products present in low abundance in complex mixtures of phenolics. The most important results obtained in this thesis will be dealt with, with respect to each other. To this end, we will discuss the prospects and limitations of the model systems and analytical methods employed. Also, new insights obtained were incorporated in the 'oxidative cascade hypothesis'. Moreover, a new, generic nomenclature for oxidized catechins is proposed, and the impact of the different levels of oxidation of tea phenolics on the sensory characteristics of tea will be addressed. Finally, it is discussed how the knowledge obtained might be used to control the phenolic profile of a black tea, and future prospects for industrial manufacturing of 'designer teas' are elaborated.

\subsection{Prospects and limitations in analysis of tea phenolics}

In this thesis, model systems were used to obtain insight in tea oxidation reactions. The advantages and disadvantages of the use of model systems compared to analysing a black tea mixture, and the use of the rapid screening method by LC-MS, based on MS/MS signature fragments, will be discussed in this subchapter.

\subsubsection{Model systems}

Model systems were used in all experimental chapters of this thesis, either to analyse the different activities of oxidative enzymes (Ch. 2\&5), or to obtain the $\mathrm{MS}^{2}$ data of compounds present in low abundance in tea (Ch. $\mathbf{3} \& \mathbf{4})$. This approach differs from most previous studies, as oxidation products were obtained under defined conditions, with a single oxidative enzyme and at the most two phenolic substrates, rather than analysing the complex mixture of oxidation products from the black tea itself.

The main advantage of using model systems for identification of compounds is the simplicity of the sample. No, or hardly any, purification steps are necessary prior to analysis of a compound, whereas for the analysis of phenolics in black tea itself, laborious separation and purification steps are required. Also, when working with model systems there will be much less batch-to-batch variation, ${ }^{1,2}$ which benefits reproducibility.

Another advantage of the use of model systems is that the initial composition is exactly known. Therefore, it is easier to determine which enzymes and substrates are involved in the formation of the resulting oxidation products. In this way, knowledge on the reactions occurring in the oxidation process can be obtained. For instance, in Chapter $\mathbf{5}$ it was shown 
that POD, and not PPO, is responsible for the hydroxylation step in the 'oxidative cascade hypothesis'.

This 'oxidative cascade hypothesis' was developed based on compounds found in black teas. ${ }^{3}$ By using model systems with tea catechins, also compounds were identified that had not been accommodated in this 'oxidative cascade hypothesis' yet, for instance the T3Ds in Chapter 3 and the DhCs in Chapter 4. By screening black tea for signature $\mathrm{MS}^{2}$ fragments of these compounds, they were indeed found to be present, albeit in low abundance. Compounds as T3Ds and DhCs are easily overlooked when analysing a complex phenolic compound mixture as black tea. By using a model system their discovery was enabled, and they could be incorporated into the 'oxidative cascade hypothesis'. In principle, a newly discovered compound in a model system might not occur in a black tea, which is valuable information by itself, i.e. to know that a certain reaction pathway does not proceed in the tea leaf matrix.

$\mathrm{MS}^{2}$ data of compounds formed in a model system, even the ones present in low abundance, can almost always easily be gathered and analysed. This is also possible in black tea, but first specific MS methods have to be developed by using model systems, to screen for the compound of interest.

Table 6.1 Advantages and disadvantages in the analysis of phenolics in model systems and in black tea compared.*

\begin{tabular}{|c|c|}
\hline Model system & Black tea \\
\hline No/less purification necessary & $\begin{array}{l}\text { Laborious separation and purification steps are } \\
\text { necessary to identify compounds }\end{array}$ \\
\hline Easy to reproduce & Natural product, reproducibility low \\
\hline $\begin{array}{l}\text { Insight can be obtained about which substrates and } \\
\text { enzymes are responsible for which reaction products }\end{array}$ & $\begin{array}{l}\text { The complex mixture and matrix make it uncertain } \\
\text { which compounds are responsible for which reactions }\end{array}$ \\
\hline $\begin{array}{l}\text { Compounds present in low abundance in tea can be } \\
\text { discovered }\end{array}$ & $\begin{array}{c}\text { Compounds present in low abundance in tea will be } \\
\text { easily overlooked }\end{array}$ \\
\hline $\begin{array}{l}\text { Not all compounds found are necessarily present in } \\
\text { tea }\end{array}$ & $\begin{array}{l}\text { All compounds identified are compounds present in } \\
\text { black tea }\end{array}$ \\
\hline $\mathrm{MS}^{2}$ data of minor compounds can be gathered easily & $\begin{array}{c}\mathrm{MS}^{2} \text { data of minor compounds can only be gathered } \\
\text { after specific MS methods are developed with model } \\
\text { systems }\end{array}$ \\
\hline $\begin{array}{l}\text { Typical matrix effects might be overlooked, e.g. } \\
\text { possible inhibitors or activators }\end{array}$ & $\begin{array}{l}\text { Realistic matrix for oxidation, effects of possible } \\
\text { inhibitors or activators are incorporated }\end{array}$ \\
\hline
\end{tabular}

${ }^{*}$ Green shading indicates advantages, red shading major disadvantages, orange shading minor disadvantages. 
When using a model system it is inevitable to compromise on the original matrix. For instance, phenolics other than catechins might be present that inhibit the tea oxidases, ${ }^{4}$ or an activity enhancing effect could be obtained from the compounds in the original matrix. ${ }^{5}$ The compounds responsible for both inhibition and enhancement of activities are not yet known. It is important to keep this in mind when applying model systems. An overview of the discussed advantages and disadvantages of the use of model systems versus black tea is shown in Table 6.1.

All in all, working with model systems is very important for obtaining insight in the reactions occurring during the oxidation of tea, albeit simplified. Black tea, however, is a too complex mixture to unveil all its secrets via model systems. Therefore, as a golden rule, it is proposed that the discovery of compounds in a model system should always be matched with a reality check in black tea. The rapid screening method with MS/MS on selected ions (Ch. 3) is an easy way to perform such a check.

\subsubsection{Rapid screening method with MS/MS on selected ions - identification by signature fragments}

In Chapter 3 a rapid screening method for black tea with UHPLC-MS/MS was proposed. In this screening method, only the MS/MS data of a priori selected ions are recorded. In this way, even compounds with low abundance were distinguished and tentatively annotated in black teas by their $\mathrm{MS}^{2}$ signature fragment(s).

To successfully determine the signature fragment(s), $\mathrm{MS}^{2}$ data of isomeric compounds should be available. Often, the $\mathrm{MS}^{2}$ data is not presented in literature, which hampers such a determination. An LC-MS ${ }^{2}$ database on tea phenolics might facilitate progress in understanding the complex oxidation processes in tea. To prevent that different LC/MS/MS settings employed blur comparisons (for instance, with high or low voltages different fragmentation patterns were obtained ${ }^{6}$ ), the settings of the reference analysis and those of the analysis of the sample of interest should be clearly correspond.

Besides comparing isomers, the reported $\mathrm{MS}^{2}$ data can also be used to extrapolate the fragmentation pattern towards corresponding compounds. For instance, phenolics with or without galloyl groups or hydroxyl groups, as performed in Chapters $\mathbf{3}$ and 5, respectively. Also fragmentation trends in certain groups of compounds can become visible, as was found previously for isoflavanoids. ${ }^{7,8}$

A practical advantage of the screening method with MS/MS on selected ions in comparison to identification by NMR spectroscopy studies is that the latter requires tedious purification procedures in order to obtain enough material for analysis. With the MS/MS method compounds can be more easily analysed in a mixture, even when present in low amounts. Also, the measurements are rapid. 
In conclusion, the MS/MS on selected ions appeared to be a good method to rapidly annotate phenolics in mixtures. Moreover, by constructing $\mathrm{MS}^{2}$ databases, this method might become even more effective.

\subsection{The 'oxidative cascade hypothesis' extended}

By information obtained from our experiments with model systems combined with rapid MS-based screenings of black tea, the 'oxidative cascade hypothesis' (from now on referred to as version 1: OCHv1), as described earlier, ${ }^{3}$ could be fine-tuned and extended into the 'oxidative cascade hypothesis version 2.0' (OCHv2). This extended version describes the increase in number of oxidation products with respect to the number of oxidation steps, as shown in Figure 6.1.

According to the OCHv1, ${ }^{3}$ the first step of oligomerization results in dimers with theaflavin (TF) type, theasinensin (TS) type, theacitrin (TC) type, or theanapthoquinone (TNQ) type interflavanic configurations. Subsequently, further oligomerization via these four types of interflavanic configurations occurs. In this thesis, three of these interflavanic configurations, which can be formed from two subunits in a single oxidation step, were defined as basic oligomerization types. The exception is TNQ, which requires a second oxidation step. TNQ is an oxidation product of TF that is formed by an intramolecular rearrangement and carbon-dioxide loss. ${ }^{9}$ Therefore, we have classified TNQs at a different level in the OCHv2. This illustrates that an oxidation reaction does not necessarily result in elongation of an oligomer. In Chapter 4 two new compounds were described to occur in tea, which can be formed by a single oxidation step, DhC $\beta$ (EC- $\left.\beta_{A B}-E C\right)$ and $\mathrm{DhC} \varepsilon$ (EC$\left.\varepsilon_{\mathrm{AB}}-\mathrm{EC}\right)$. Therefore, these molecules are incorporated in the first level of the OCHv2 as basic oligomerization types (Fig 6.1).

Other oxidation products newly found in tea in our research are T3Ds (Ch. 3). Just like the TNQs, they require two oxidation steps to be formed. T3Ds are trimers of catechins in which the third catechin moiety is coupled to the interflavanic configuration of a $\mathrm{TF}$, creating an interflavanic configuration type other than the basic oligomerization types. Therefore, we have distinguished two types of oligomerization, oligomerization via basic interflavanic configurations and oligomerizations in which the preformed basic interflavanic configuration participates by constituting a scaffold onto which a third catechin moiety is superimposed. The latter type of oligomerization we have defined as a tripartite oligomerization type.

In the OCHv1, hydroxylation would be the next level after oligomerization. However, also hydroxylated dimers have been found, ${ }^{10,11}$ and no evidence has been found so far that after hydroxylation no more oligomerization can take place. In the OCHv2, the hydroxylation reaction is, therefore, put at the same level as the oligomerizations and 
intramolecular rearrangements. Therefore, the second level in the OCHv2, which is defined by two or more oxidation steps, can be divided into four oxidation reactions: (i) basic oligomerization, (ii) tripartite oligomerization, (iii) intramolecular rearrangements, and (iv) hydroxylation (Fig 6.1). All these different oxidation reactions can occur in random order, resulting in a large variety of oxidation products.

Level 3 of the OCHv1 involved the redox equilibria of the polyhydroxylated oligomers with their quinones. These equilibria are presumably caused by coupled oxidation, hence enzymes are not directly involved. Therefore, this level was not further discussed in this research. Level 4 is not taken into account in the OCHv2 as well, because the OCHv2 focusses only on the enzymatic oxidative processes, whereas level 4 represented either glycosylation or methylation. Besided that, the occurrence of these reactions has not been evidenced in tea so far.

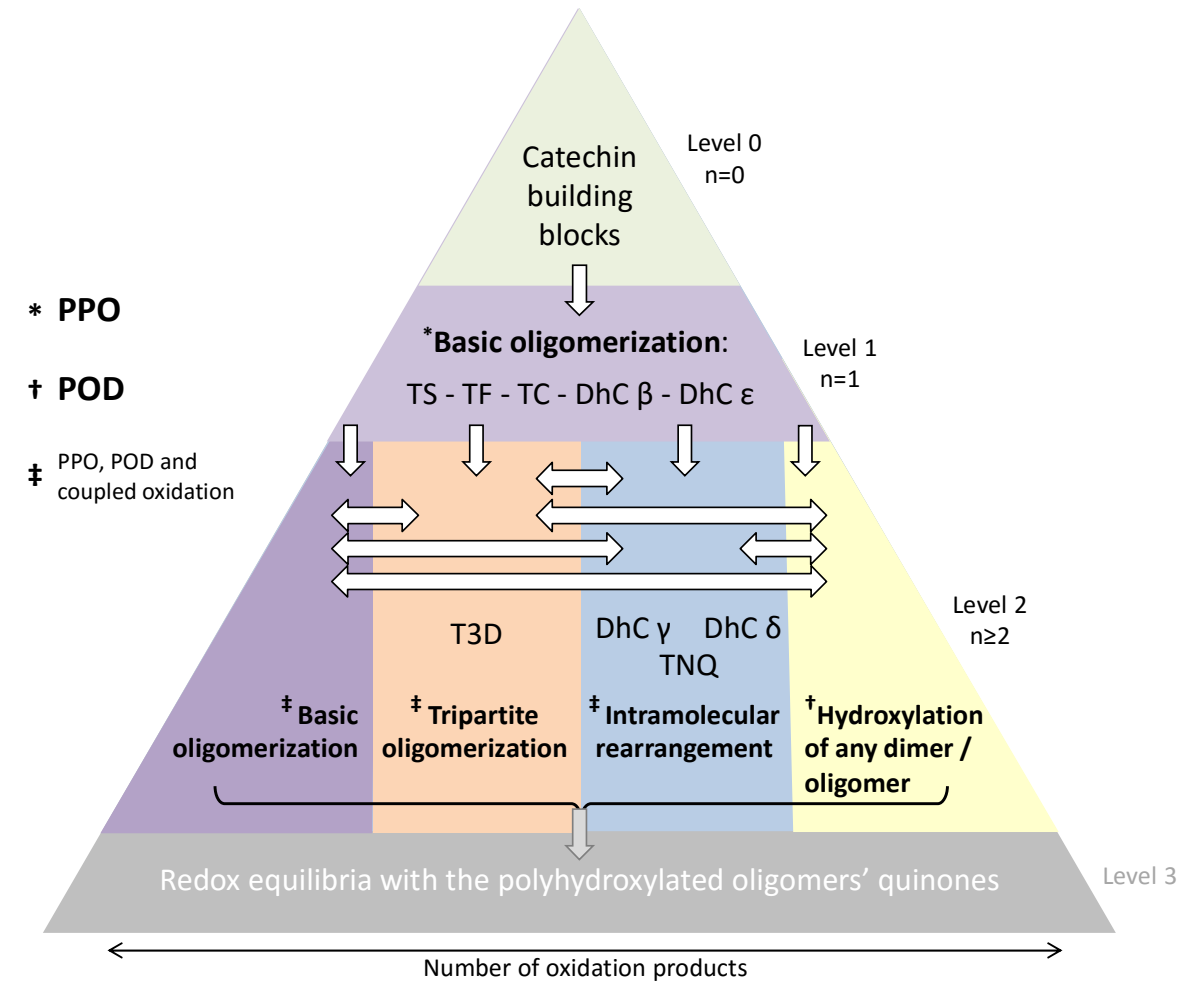

Figure 6.1 The 'oxidative cascade hypothesis v2.0', extended version of the 'oxidative cascade hypothesis'3. $\mathrm{n}=$ number of oxidation steps. Arrows indicate the possible directions of the oxidation reactions. The enzymes responsible are indicated. 
The first level of the OCHv2, the formation of the dimers, will be mainly catalysed by PPO, because it is known that POD is not active yet in the first stages of oxidation of phenolics. ${ }^{12}$ The reason for this is that first $\mathrm{H}_{2} \mathrm{O}_{2}$ has to be formed during the oxidation of the catechins by PPO. ${ }^{13-15}$ The enzyme activities in the second level of the OCHv2 were not specified yet for the further oligomerization via basic and tripartite types, and intramolecular rearrangements. It will be a combination of the activities of both PPO and POD, while coupled oxidation ${ }^{16,17}$ will also play a role. The enzyme responsible for the hydroxylation in the second level was still unknown as well. In Chapter $\mathbf{5}$ we showed that in fact POD is the enzyme responsible for the hydroxylation. The enzymatic activities are incorporated in the OCHv2 (Fig 6.1).

With the OCHv2, the knowledge on tea oxidation has become more complete. It clearly shows, taking into account the theoretical numbers of isomers for DhCs up to tetramers (Ch. 4) and those for TFs with up to 5 extra hydroxyl groups (Ch. 5), how the number of unique oxidation products from the limited set of starting compounds (the catechins) can become enormous, at least the predicted 10,000. ${ }^{3}$ The collective noun 'thearubigins' (TRs) does not do justice to the plenitude of oxidation products obtained. Furthermore, the original definition of thearubigins, the ethyl acetate insoluble phenolics from tea, ${ }^{18,19}$ is hard to keep up, because it is unclear whether the newly discovered tea phenolics are ethyl acetate insoluble. To address the different unique oxidation products systematically, we propose here a generic nomenclature that incorporates the different aspects of the OCHv2. The noun TR should be replaced by this nomenclature.

\subsection{Towards an extendable nomenclature for oxidized tea phenolics}

The OCHv2 describes the different oxidation products of catechins in tea, but the current nomenclature runs short in classifying oxidized phenolics systematically. The current names neither provide information on the type of interflavanic configuration, nor on the combination of constituent catechins and the degree of oligomerization. In Chapter 4, a new nomenclature for the dehydrocatechins was introduced, which accounted for the interflavanic configuration and degree of oligomerization. In this subchapter, we extend this nomenclature, including the newly found interflavanic configurations described in this thesis. The nomenclature will cover the catechin oxidation products in tea, but might be further extended to other phenolic oxidation products. 


\subsubsection{Compounds formed by linear oligomerization}

In tea, the oxidation products are all built up from only 4 different catechin subunits and their epimers. As for the dehydrocatechins (Ch. 4), it is proposed to mention the constituent subunits in the nomenclature, although it should be considered that the subunits often change structure after reaction. For instance, in TF the EC subunit can still be easily recognized, but the B-ring of the EGC subunit is restructured into the seven-membered benzotropolone ring. For simplicity, only the name of the unreacted, parental catechin subunits is referred to in the nomenclature. The restructuring of the subunits will be inherent to the type of interflavanic configuration formed, which is incorporated in the nomenclature.

In between two subunits, the code for the interflavanic configuration is stated. In Table 6.2 an overview of the different interflavanic configurations in black tea, known so far, is shown. Extending from the nomenclature of the dehydrocatechins and procyanidins, for which the interflavanic configurations are referred to with $\alpha$ to $\varepsilon$, other Greek letters are given to other configurations (Table 6.2). For instance, the seven-membered benzotropolone ring formed as interflavanic configuration in theaflavins and theaflavates will now be referred to as a $\zeta$-linkage. The Greek letter codes are given arbitrary to the different interflavanic configurations, any newly discovered interflavanic configuration can obtain a next letter of the Greek alphabet.

In Chapter 4, the nomenclature starts with the terminal subunit. The dehydrocatechins and procyanidins both had a clear terminal side from which to start the nomenclature, because they oligomerized always as $\mathrm{A} \rightarrow \mathrm{B}$ or $\mathrm{A} \rightarrow \mathrm{C}$, respectively. For most of the other tea oxidation products this is less straight forward. Most oxidation products are coupled B-ring to B-ring, ${ }^{20,21}$ which leaves no unique terminal side. Therefore, it is proposed to start the nomenclature alphabetically at the side with the lowest letter from the Greek alphabet. In dimers only one interflavanic configuration (hence Greet letter) is present. Therefore, the nomenclature will start at the side with subunit (E)GCg $>(\mathrm{E}) \mathrm{Cg}>(\mathrm{E}) \mathrm{GC}>(\mathrm{E}) \mathrm{C}$.

The letter of the rings (A,B or $\mathrm{C}$ ) interlinked should be indicated as a subscript (terminal/starting unit $\rightarrow$ extension unit) of the Greek letter, as for the dehydrocatechins (Ch. 4). For instance, theaflavin will be EGC- $\zeta_{\mathbf{B B}}-\mathbf{E C}$ (Fig. 6.2). Oligomerization of the dehydrocatechins occurred linearly, always A-ring to B-ring. In this case, the nomenclature can just extend linearly as well. 


\subsubsection{Compounds formed by branched oligomerization}

In tea also oligomers are present that are not linear. The tripartite oligomerization type can be seen as a kind of branching. In case of galloylation of the initial catechins, further oligomerization with this trihydroxylic benzene ring, referred to as the D-ring, can occur as well. The latter can also been seen as branching.

It is proposed in this nomenclature to write the type of branching between parentheses next to the subunit from which the branching occurs, in analogy to the nomenclature of polysaccharides. ${ }^{22}$ In case of a tripartite oligomerization type in which the branching is superimposed on a basic interflavanic configuration, only the code for the T3D interflavanic configuration $(\kappa)$ is included in the nomenclature. The $\zeta$ of the TF configuration is left out. Therefore, T3D will be referred to as: EGC- $\boldsymbol{\kappa}_{\mathbf{B B B}}(\mathbf{E C})-\mathbf{E C}$ (Fig 6.2).

Branching from a galloyl-group (D-ring) will be mentioned between parentheses next to the subunit with the galloyl-group. The ' $D$ ' in the subscript of the configuration code indicates that it branches from the galloyl-group. For instance, a TFg with a theaflavatelike branch of one EC at the galloyl-group of the initial EGCg, would be referred to as:

\section{$\operatorname{EGCg}\left(\zeta_{D B}-E C\right)-\zeta_{B B}-E C($ Fig 6.2)}
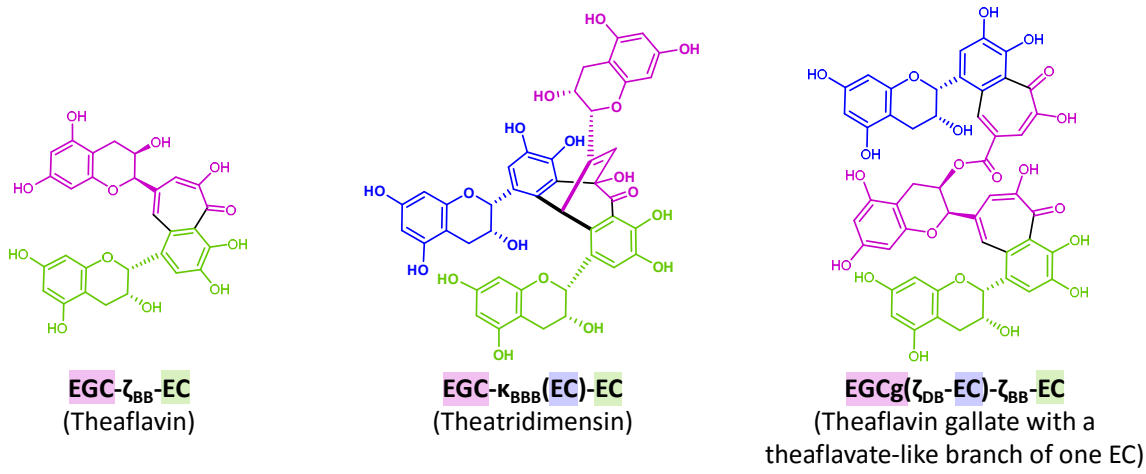

Figure 6.2 Examples of the nomenclature proposed to systematically represent oligomeric, oxidized phenolics.

\subsubsection{Hydroxylation}

Besides oligomerization and rearrangements, also hydroxylation is an oxidation reaction described for tea in the OCHv2. Incorporation of the hydroxylation step in the nomenclature, however, left us with a dilemma. As soon as a hydroxyl group is added to an oligomer, one of the subunits actually changed. For instance, an EC subunit in the oligomer could be changed into an EGC subunit. It was decided to leave out the hydroxylation step from the nomenclature for now. 
Table 6.2 Overview of the proposed nomenclature for the different interflavanic configurations, including their type of oxidation, in the oxidation products of tea.

\begin{tabular}{|c|c|c|c|c|}
\hline \multicolumn{3}{|c|}{ Interflavanic configuration } & \multirow{2}{*}{ Trivial name } & \multirow{2}{*}{ Oxidation type } \\
\hline Code & Structure $^{*}$ & $\begin{array}{l}\text { Rings } \\
(T \rightarrow E)\end{array}$ & & \\
\hline$\alpha$ & & B-B & Dehydrotheasinensin C & $\begin{array}{c}\text { Basic } \\
\text { oligomerization }\end{array}$ \\
\hline$\alpha$ & & $\zeta-\zeta$ & Bistheaflavin A & $\begin{array}{c}\text { Basic } \\
\text { oligomerization }\end{array}$ \\
\hline$\alpha$ & & $\mathrm{A}-\mathrm{C}$ & Procyanidin A & Miscellaneous ${ }^{\dagger}$ \\
\hline$\beta$ & & A-B & Dehydrocatechin B4 & $\begin{array}{c}\text { Basic } \\
\text { oligomerization }\end{array}$ \\
\hline$\beta$ & & B-B & Theasinensin & $\begin{array}{c}\text { Basic } \\
\text { oligomerization }\end{array}$ \\
\hline$\beta$ & & $\mathrm{A}-\mathrm{C}$ & Procyanidin B & Miscellaneous ${ }^{\dagger}$ \\
\hline$\gamma$ & & A-B & Dehydrocatechin A & $\begin{array}{l}\text { Intramolecular } \\
\text { rearrangement }\end{array}$ \\
\hline$\gamma$ & & B-B & Dehydrotheasinensin E & $\begin{array}{l}\text { Intramolecular } \\
\text { rearrangement }\end{array}$ \\
\hline$\delta$ & & A-B & Dehydrocatechin VII & $\begin{array}{l}\text { Intramolecular } \\
\text { rearrangement }\end{array}$ \\
\hline
\end{tabular}


General discussion

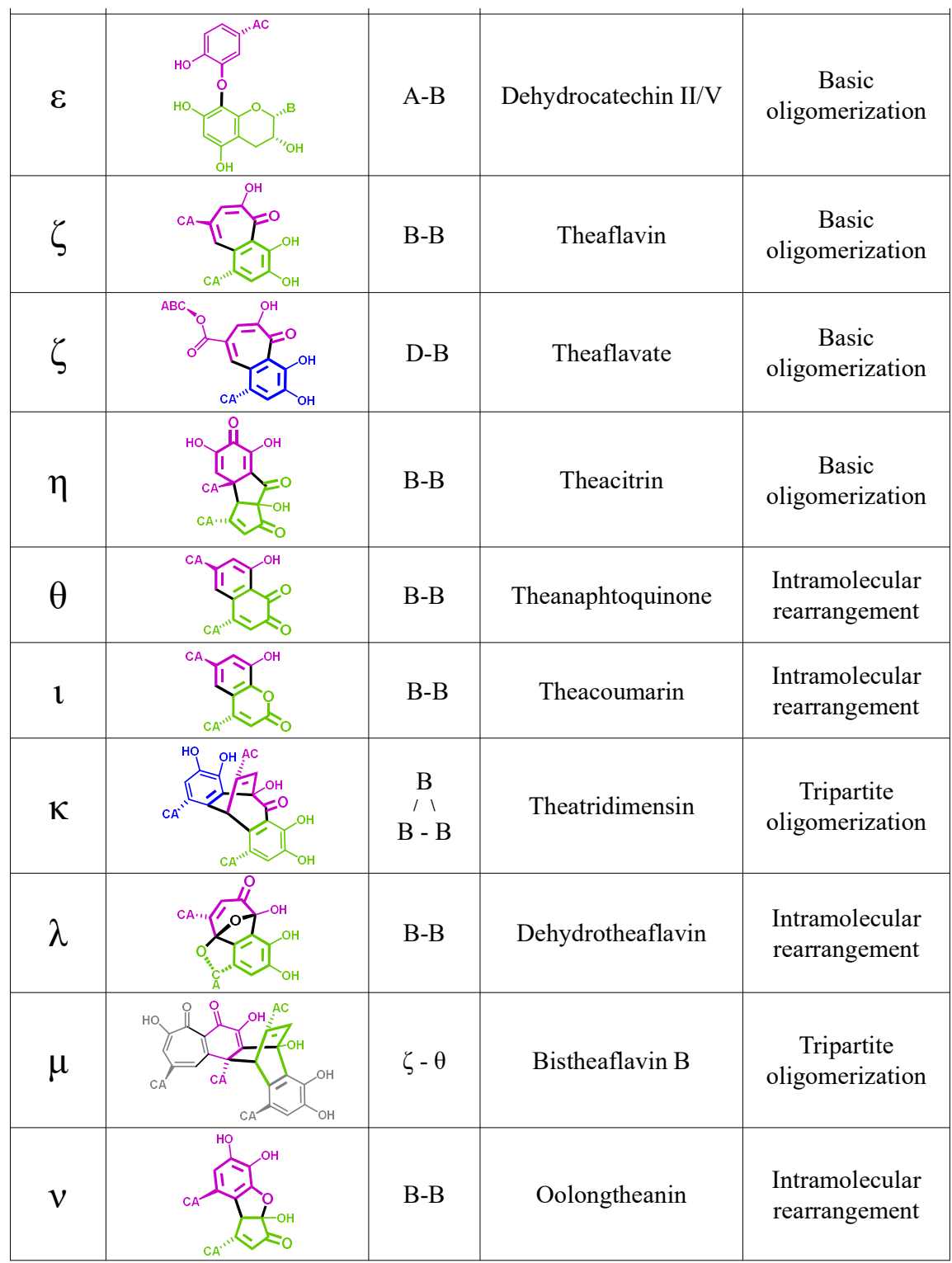

* The different subunits are indicated in green and pink, branched subunits in blue, subunits not involved in the binding in grey, and new bonds in black. The interflavanic configuration is shown bold.

† Procyanidins are classified in the miscellaneous category, because there is no evidence that they are formed during the oxidation. 


\subsection{Extrapolating the 'oxidative cascade hypothesis v2.0' to sensory characteristics}

As explained above, oligomerization, intramolecular rearrangements and hydroxylation of catechins can occur during tea oxidation. The possible consequences of these different oxidation reactions on the sensory characteristics, astringency and bitterness in particular, will be discussed in this subchapter.

\subsubsection{Astringency}

The astringency of the different catechin oxidation products depends on (i) the degree of polymerization (DP), ${ }^{23-26}$ (ii) the conformational flexibility, ${ }^{25}$ (iii) the degree of hydroxylation, ${ }^{23,24}$ (iv) the hydrophobicity, ${ }^{25}$ and (v) the galloyation. ${ }^{23}$ Theoretically, the further the 'oxidative cascade' proceeds, the higher the astringency expected, because of the increase of the DP and of hydroxylation. However, there will be an optimum in this. For instance, it was shown that at too high molecular weight $(>3400 \mathrm{Da})$ interaction was hampered (with globular proteins), ${ }^{26}$ probably due to a decreased conformational flexibility. Therefore, the effect of DP on astringency is difficult to predict beforehand. An example of decreasing the astringency of tea was shown by addition of tannases, which decreased the extent of galloyation. ${ }^{27,28}$

As an additional experiment, an estimation of the astringency of various tea phenolics was performed, by analysing their binding affinity to proteins. A method including an ultrafiltration step prior to mass spectrometric analysis was used, following the procedure outlined by ${ }^{29}$, with a phenolics concentration range of $(0.004-0.5 \mathrm{mg} / \mathrm{mL})$, and with the exception of using MS/MS on selected ions (Ch. 3) instead of SRM in the mass spectrometric analysis. The results can be used to estimate a binding affinity, which is indicative for astringency. ${ }^{30}$ Prior to ultrafiltration, the proteins were exposed to phenolics. $\beta$-casein was used as protein, because the random coil structure resembles that of salivary proteins, although the proline content (a driver for interaction ${ }^{26}$ ) of $\beta$-casein is lower than that of salivary proteins. After incubation, the samples were ultrafiltrated to separate the unbound phenolics from the protein and protein-bound phenolics. The unbound phenolics were subsequently semi-quantified by LC-MS. The amount of bound phenolics was calculated from the differences between the concentration of initially added (free) phenolics and the concentration of unbound phenolics after incubation with $\beta$-casein. Incubations were performed with different concentrations of phenolics at a fixed protein concentration. When plotting the bound phenolics versus the initial concentration of phenolics, the slope of the adsorption isotherm is defined as the binding affinity ( $\mathrm{K}_{\text {apparent }}$ ). ${ }^{31}$ Figure 6.3 shows an example with T3D, with the LC-MS signal before and after incubation with $\beta$-casein, together with the resulting adsorption isotherm. After incubation with $\beta$-casein, the T3D 
almost completely disappeared, hence it was bound to the protein. The slope of the binding curve showed that the binding affinity of T3D with $\beta$-casein was almost $7500 \mathrm{M}^{-1}$.

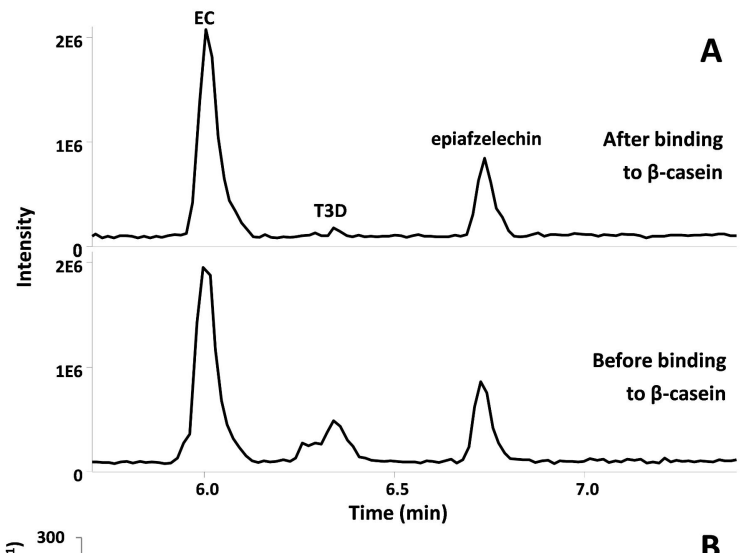

A

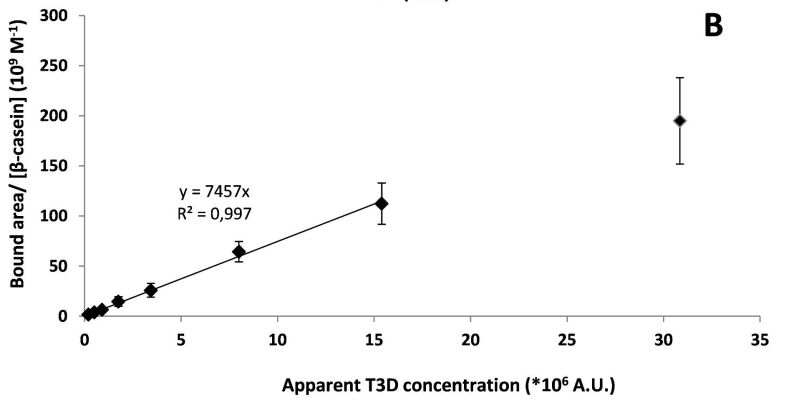

Figure 6.3 Example of determination of the binding affinity of T3D to $\beta$-casein, with the ultrafiltration - MS binding assay. A) The MS trace of the incubation before and after incubation with $\beta$-casein. B) The adsorption isotherm of T3D with $\beta$-casein.

The resulting preliminary binding affinities of the various semi-purified tea phenolics are shown in Figure 6.4. The preliminary results showed T3D to have the highest binding affinity, which might be explained by the higher degree of polymerization and higher number of hydroxyl groups, compared to the other phenolics tested. EC, on the contrary, showed a low binding affinity, because it is a monomer. ${ }^{24}$ For the dimeric tea compounds, $\mathrm{DhC} \varepsilon_{\mathrm{AB}}\left(\mathrm{EC}-\varepsilon_{\mathrm{AB}}-\mathrm{EC}\right)$ gave the highest binding affinity, which might be explained by its high rotational freedom. $\mathrm{DhC} \delta_{\mathrm{AB}}\left(\mathrm{EC}-\delta_{\mathrm{AB}}-\mathrm{EC}\right)$ has much less rotational freedom than most other dimeric oxidation products tested, which might explain its relatively low binding affinity. TS showed the lowest binding affinity, which might be explained by the hampered rotational freedom of the two EGC subunits, due to steric hindrance. TNQ had a binding affinity for $\beta$-casein being almost double that of TF. Interestingly, the number of hydroxyl groups and the molecular flexibilities of the two molecules, do not differ very much. A 
possible explanation might be that the benzotropolone ring of TF is less efficient in engaging in stacking interactions than the 1,2-naphtoquinone moiety in TNQ, although the difference in planarity is not very large. It should be noted that the results in Figure 6.4 are preliminary.

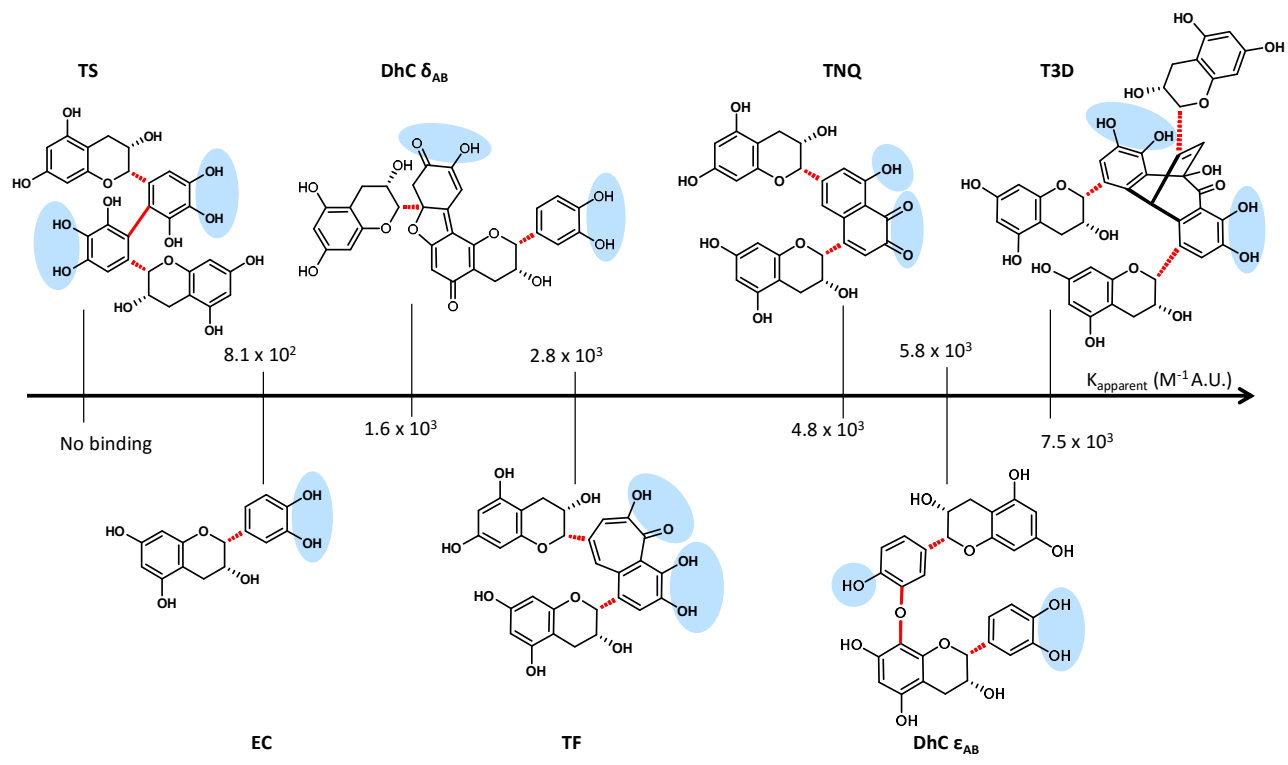

Figure 6.4 A schematic overview relating the binding affinity ( $\left.K_{\text {apparent }}\right)\left(M^{-1}\right)$ determined to the molecular structure of individual oxidation products from tea. Their proposed hydrogen binding spots on their B-ring are indicated with blue shading, the bonds with rotational freedom are indicated in red and bold. ${ }^{23-25}$

\subsubsection{Bitterness}

Catechins are known to give a bitter taste, ${ }^{32,33}$ but the bitterness of oxidation products in black tea is less well investigated. ${ }^{34}$ Nevertheless, bitterness is also an important quality parameter of tea, with varying preference of consumers around the world for bitterness. The perception of bitterness is caused by the activation of bitter taste receptors (T2Rs) in the mouth. ${ }^{35}$ Cell-based assays have been used to determine, in vitro, the intrinsic bitterness of different phenolic compounds. ${ }^{36}$ These studies suggested that a higher degree of hydroxylation of isoflavanoids caused a higher binding to T2Rs. ${ }^{36}$ With modelling studies it was found that the position of the hydroxyl groups on the isoflavanoids was important as well. $^{37}$ 
These observations indicate that the hydroxylation step in the OCHv2 is likely to enhance the bitterness of tea. It might, therefore, be attractive to either circumvent or enhance the hydroxylation step catalysed by POD in a (still to be developed) controlled oxidation process of tea leaves, to influence the final bitterness of the tea. Also, it was suggested that steric hindrance was of influence for the binding of bitter compounds to the bitter receptors. ${ }^{38}$ Hence, oligomerization of catechins might reduce bitterness, as the molecules might become too large to fit the tastant-binding pockets of T2Rs. The dimeric TFs do not comply with this idea, as they had a lower $\mathrm{EC}_{50}$ value, thus are more bitter, than monomeric catechins. ${ }^{32,39}$ It is evident that the influence of oligomerization on bitterness requires further attention.

\subsection{Towards teas with a designer phenolics profile}

By making use of model systems, the OCHv1 describing the oxidation reactions in tea has been extended with new types of interflavanic configurations, and the role of PPO and POD has become more clear, resulting in the OCHv2. With this knowledge, opportunities for designing a specific phenolics profile in black tea might come within reach. The options to control oxidation reactions can be exerted at the oxidative enzyme level and / or at the catechin level. For this, it might be necessary to separate the oxidative enzymes and catechins prior to the oxidation.

For a 'mild' separation process of oxidative enzymes and catechins, the following criteria should be met: (i) Both the enzymatic activities and the phenolic compounds should be obtained in their native states, i.e. active and non-oxidized, respectively, and (ii) no 'harsh' chemicals should be used (e.g. some organic solvents, non-food grade, etc.). In this research, both the oxidative enzymes and the catechins have been extracted separately from fresh tea leaves. In Chapter 2, a phenolic extract was prepared from green tea leaves by extraction with boiling water. In this way, the oxidation of the catechins was prevented by irreversibly denaturing the oxidative enzymes. Therefore, this method does not apply as 'mild', because it does not fit with the first criterion. In Chapter 5, an enzyme extract was prepared from fresh tea leaves by blending the tea leaves with buffer solution. The catechins were removed from the extract by precipitation with PVPP, in order to prevent interference with absorbance measurements in activity assays. This separation method applies as 'mild', because both catechins (albeit adsorbed to PVPP ${ }^{40}$ ) and oxidative enzymes are recovered in their native states. In principle, the catechins might be recovered from the PVPP,${ }^{41}$ for instance, by extraction with ethanol. ${ }^{42}$

An alternative 'mild' disruption method of biological tissues is pulsed electric field (PEF). ${ }^{43}$ By application of an electric field for short durations (a few to several hundred milliseconds) cell membrane permeability is induced, a phenomenon called 
'electroporation', without increasing temperature. ${ }^{44,45}$ Although not reported in the particular PEF study on tea leaves, ${ }^{46} 97 \%$ of the oxidative enzyme activities were retained in the leaf material (no further data shown). However, only $30 \%$ of the catechins was recovered in the surrounding liquid. ${ }^{46}$ Thus, under the conditions used, PEF does not seem to be a promising treatment for mild disclosure.

In Chapter 2, it was found that incubation of green tea phenolics with only tyrosinase (PPO activity) yielded a higher and more stable TF content than oxidation with laccase or the endogenous tea enzymes. In Chapter 5 it was shown that only POD is responsible for the hydroxylation step in the OCHv2. Taking this together, this showed that two of the main reactions occurring in black tea oxidation, the first oligomerization step (dimerization) and the hydroxylation step, are catalysed by two different enzymes. This knowledge reveals attractive opportunities for controlling the oxidation reactions, based on uncoupling the two different oxidative activities, by either enhancing the desired activity or inhibiting the undesired one. For instance, if a less bitter tea is preferred, POD activity could be inhibited/removed. This prevents hydroxylation of the tea oligomers, and consequently reduces bitterness. ${ }^{36}$ Alternatively, pure exogenous oxidases could be added to an extract of green tea catechins, as was shown with tyrosinase and laccase in Chapter 2. Adding exogenous tyrosinase to the leaves during oxidation is also an option. ${ }^{47}$ The use of the endogenous enzymes from tea would, however, be preferred. Therefore, the biochemical characteristics of tea oxidases should be determined into more detail, in order to further establish the mild separation of the enzymes and phenolics of tea leaves.

The individual catechins and combinations of catechins are known to result in different oxidation products. $^{21}$ Also the rate of the reactions is influenced by the catechin composition. This was shown in Chapter 2 where the reactivity of PPO (tyrosinase) on only EC was compared with that on EC in combination with EGC. The reactivity on EC was shown to be 17 times higher in combination with EGC than with EC alone. Apparently, formation of TFs is favoured over formation of DhCs. The catechin composition is, therefore, an important tool to modulate the oxidation process and fractionation of the catechin stream after mild disclosure seems desirable. Nevertheless, it should be noted that fractionation of tea catechins at industrial scale is not yet economically feasible. The mild disclosure and controlled oxidation of tea is schematically represented in Figure 6.5. 


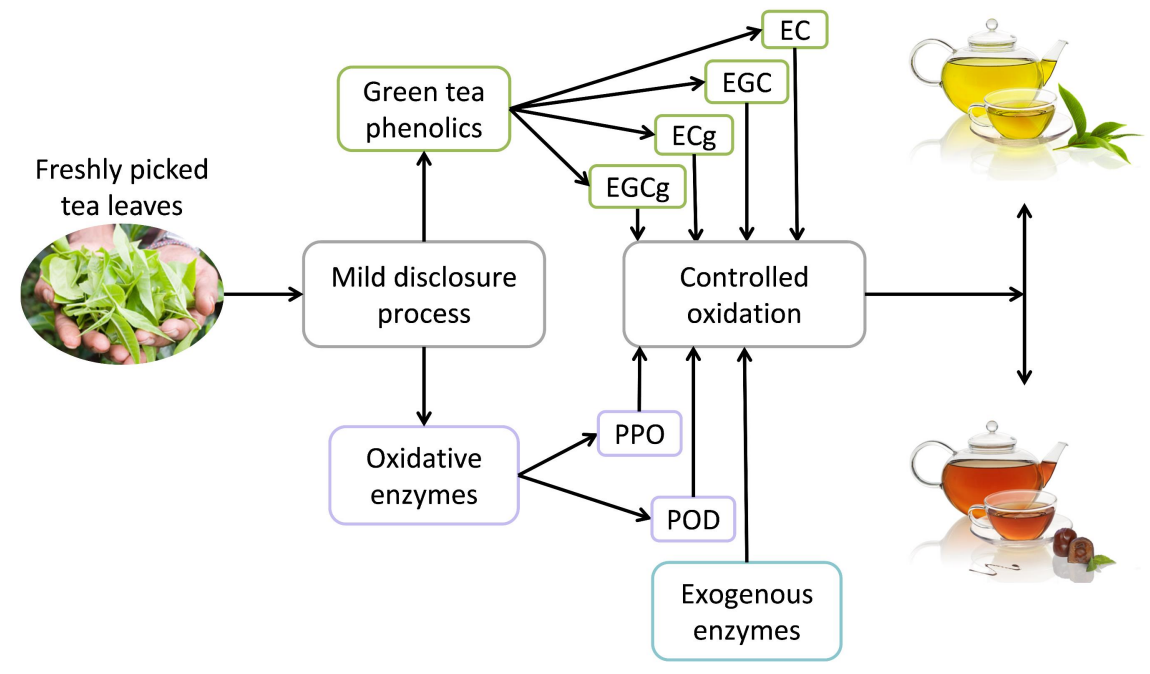

Figure 6.5 Schematic representation of refining tea oxidation by mild disclosure of oxidative enzymes and phenolics.

\subsection{References}

1. Chen, Y.; Jiang, Y.; Duan, J.; Shi, J.; Xue, S.; Kakuda, Y. Variation in catechin contents in relation to quality of 'Huang Zhi Xiang' Oolong tea (Camellia sinensis) at various growing altitudes and seasons. Food Chemistry 2010, 119, 648-652.

2. Lin, Y. L.; Juan, I. M.; Chen, Y. L.; Liang, Y. C.; Lin, J. K. Composition of polyphenols in fresh tea leaves and associations of their oxygen-radical-absorbing capacity with antiproliferative actions in fibroblast cells. Journal of Agricultural and Food Chemistry 1996, 44, 1387-1394.

3. Kuhnert, N.; Drynan, J. W.; Obuchowicz, J.; Clifford, M. N.; Witt, M. Mass spectrometric characterization of black tea thearubigins leading to an oxidative cascade hypothesis for thearubigin formation. Rapid Communications in Mass Spectrometry 2010, 24, 3387-3404.

4. Kuijpers, T. F. M.; Van Herk, T.; Vincken, J. P.; Janssen, R. H.; Narh, D. L.; Van Berkel, W. J. H.; Gruppen, H. Potato and mushroom polyphenol oxidase activities are differently modulated by natural plant extracts. Journal of Agricultural and Food Chemistry 2014, 62, 214-221.

5. Stodt, U. W.; Blauth, N.; Niemann, S.; Stark, J.; Pawar, V.; Jayaraman, S.; Koek, J.; Engelhardt, U. H. Investigation of processes in black tea manufacture through model fermentation (oxidation) experiments. Journal of Agricultural and Food Chemistry 2014, 62, 7854-7861.

6. Yassin, G. H.; Koek, J. H.; Kuhnert, N. Identification of trimeric and tetrameric flavan-3-ol derivatives in the SII black tea thearubigin fraction of black tea using ESI-tandem and MALDI-TOF mass spectrometry. Food Research International 2014, 63, 317-327.

7. Simons, R.; Vincken, J. P.; Bakx, E. J.; Verbruggen, M. A.; Gruppen, H. A rapid screening method for prenylated flavonoids with ultra-high-performance liquid chromatography/ electrospray ionisation mass spectrometry in licorice root extracts. Rapid Communications in Mass Spectrometry 2009, 23, 3083-3093. 
8. Simons, R.; Vincken, J. P.; Bohin, M. C.; Kuijpers, T. F. M.; Verbruggen, M. A.; Gruppen, H. Identification of prenylated pterocarpans and other isoflavonoids in Rhizopus spp. elicited soya bean seedlings by electrospray ionisation mass spectrometry. Rapid Communications in Mass Spectrometry 2011, 25, 55-65.

9. Tanaka, T.; Betsumiya, Y.; Mine, C.; Kouno, I. Theanaphthoquinone, a novel pigment oxidatively derived from theaflavin during tea-fermentation. Chemical Communications 2000, 1365-1366.

10. Yassin, G. H.; Koek, J. H.; Jayaraman, S.; Kuhnert, N. Identification of novel homologous series of polyhydroxylated theasinensins and theanaphthoquinones in the SII fraction of black tea thearubigins using ESI/HPLC tandem mass spectrometry. Journal of Agricultural and Food Chemistry 2014, 62, 9848-9859.

11. Kuhnert, N.; Clifford, M. N.; Müller, A. Oxidative cascade reactions yielding polyhydroxy-theaflavins and theacitrins in the formation of black tea thearubigins: Evidence by tandem LC-MS. Food and Function 2010, 1, 180-199.

12. Mahanta, P. K.; Boruah, S. K.; Boruah, H. K.; Kalita, J. N. Changes of polyphenol oxidase and peroxidase activities pigment composition of some manufactured black teas (Camellia, sinensis L.). Journal of Agricultural and Food Chemistry 1993, 41, 272-276.

13. Subramanian, N.; Venkatesh, P.; Ganguli, S.; Sinkar, V. P. Role of polyphenol oxidase and peroxidase in the generation of black tea theaflavins. Journal of Agricultural and Food Chemistry 1999, 47, 2571-2578.

14. Richard-Forget, F. C.; Gauillard, F. A. Oxidation of chlorogenic acid, catechins, and 4-methylcatechol in model solutions by combinations of pear (Pyrus communis cv. williams) polyphenol oxidase and peroxidase: A possible involvement of peroxidase in enzymatic browning. Journal of Agricultural and Food Chemistry 1997, 45, 2472-2476.

15. Jiang, Y.; Miles, P. W. Generation of $\mathrm{H}_{2} \mathrm{O}_{2}$ during enzymic oxidation of catechin. Phytochemistry 1993, 33 29-34.

16. Tanaka, T.; Mine, C.; Inoue, K.; Matsuda, M.; Kouno, I. Synthesis of theaflavin from epicatechin and epigallocatechin by plant homogenates and role of epicatechin quinone in the synthesis and degradation of theaflavin. Journal of Agricultural and Food Chemistry 2002, 50, 2142-2148.

17. Bajaj, K. L.; Anan, T.; Tsushida, T.; Ikegaya, K. Effects of (-)-epicatechin on oxidation of theaflavins by polyphenol oxidase from tea leaves. Agricultural and Biological Chemistry 1987, 51, 1767-1772.

18. Roberts, E. A. H.; Smith, R. F. Spectrophotometric measurements of theaflavins and thearubigins in black tea liquors in assessments of quality in teas. The Analyst 1961, 86, 94-98.

19. Roberts, E. A. H.; Cartwright, R. A.; Oldschool, M. The phenolic substances of manufactured tea. I. Fractionation and paper chromatography of water-soluble substances. Journal of Agricultural and Food Chemistry 1957, 8, 72-80.

20. Yassin, G. H.; Koek, J. H.; Kuhnert, N. Model system-based mechanistic studies of black tea thearubigin formation. Food Chemistry 2015, 180, 272-279.

21. Drynan, J. W.; Clifford, M. N.; Obuchowicz, J.; Kuhnert, N. The chemistry of low molecular weight black tea polyphenols. Natural Product Reports 2010, 27, 417-462.

22. Polysaccharide nomenclature. Recommendations 1980. IUB-IUPAC Joint Commission on Biochemical Nomenclature (JCBN). Journal of Biological Chemistry 1982, 257, 3352-3354.

23. Canon, F.; Giuliani, A.; Paté, F.; Sarni-Manchado, P. Ability of a salivary intrinsically unstructured protein to bind different tannin targets revealed by mass spectrometry. Analytical and Bioanalytical Chemistry 2010, 398, 815-822.

24. Plet, B.; Delcambre, A.; Chaignepain, S.; Schmitter, J. M. Affinity ranking of peptide-polyphenol noncovalent assemblies by mass spectrometry approaches. Tetrahedron 2015, 71, 3007-3011.

25. Spencer, C. M.; Cai, Y.; Martin, R.; Gaffney, S. H.; Goulding, P. N.; Magnolato, D.; Lilley, T. H.; Haslam, E. Polyphenol complexation-some thoughts and observations. Phytochemistry 1988, 27, 2397-2409. 
26. De Freitas, V.; Mateus, N. Nephelometric study of salivary protein-tannin aggregates. Journal of the Science of Food and Agriculture 2002, 82, 113-119.

27. Chandini, S. K.; Rao, L. J.; Gowthaman, M. K.; Haware, D. J.; Subramanian, R. Enzymatic treatment to improve the quality of black tea extracts. Food Chemistry 2011, 127, 1039-1045.

28. Raghuwanshi, S.; Misra, S.; Saxena, R. K. Enzymatic treatment of black tea (ctc and kangra orthodox) using penicillium charlesii tannase to improve the quality of tea. Journal of Food Processing and Preservation 2012.

29. Bohin, M. C.; Vincken, J. P.; Van Der Hijden, H. T. W. M.; Gruppen, H. Efficacy of food proteins as carriers for flavonoids. Journal of Agricultural and Food Chemistry 2012, 60, 4136-4143.

30. Jöbstl, E.; O'Connell, J.; Fairclough, J. P. A.; Williamson, M. P. Molecular model for astringency produced by polyphenol/protein interactions. Biomacromolecules 2004, 5, 942-949.

31. Bohin, M. C. Food proteins as potential carriers for phenolics - Chapter 4. Wageningen University, Ede, The Netherlands, 2013.

32. Narukawa, M.; Noga, C.; Ueno, Y.; Sato, T.; Misaka, T.; Watanabe, T. Evaluation of the bitterness of green tea catechins by a cell-based assay with the human bitter taste receptor hTAS2R39. Biochemical and Biophysical Research Communications 2011, 405, 620-625.

33. Chen, Q.; Guo, Z.; Zhao, J. Identification of green tea's (Camellia sinensis (L.)) quality level according to measurement of main catechins and caffeine contents by HPLC and support vector classification pattern recognition. Journal of Pharmaceutical and Biomedical Analysis 2008, 48, 1321-1325.

34. Drewnowski, A.; Gomez-Carneros, C. Bitter taste, phytonutrients, and the consumer: A review. American Journal of Clinical Nutrition 2000, 72, 1424-1435.

35. Ma, W.; Guo, A.; Zhang, Y.; Wang, H.; Liu, Y.; Li, H. A review on astringency and bitterness perception of tannins in wine. Trends in Food Science and Technology 2014, 40, 6-19.

36. Roland, W. S. U.; Vincken, J. P.; Gouka, R. J.; Van Buren, L.; Gruppen, H.; Smit, G. Soy isoflavones and other isoflavonoids activate the human bitter taste receptors hTAS2R14 and hTAS2R39. Journal of Agricultural and Food Chemistry 2011, 59, 11764-11771.

37. Roland, W. S. U.; Van Buren, L.; Gruppen, H.; Driesse, M.; Gouka, R. J.; Smit, G.; Vincken, J. P. Bitter taste receptor activation by flavonoids and isoflavonoids: Modeled structural requirements for activation of hTAS2R14 and hTAS2R39. Journal of Agricultural and Food Chemistry 2013, 61, 10454-10466.

38. Belitz, H.-D.; Wieser, H. Bitter compounds: Occurrence and structure-activity relationships. Food Reviews International 1985, 1, 271-354.

39. Yamazaki, T.; Sagisaka, M.; Ikeda, R.; Nakamura, T.; Matsuda, N.; Ishii, T.; Nakayama, T.; Watanabe, T. The human bitter taste receptor hTAS2R39 is the primary receptor for the bitterness of theaflavins. Bioscience, Biotechnology and Biochemistry 2014, 78, 1753-1756.

40. Laborde, B.; Moine-Ledoux, V.; Richard, T.; Saucier, C.; Dubourdieu, D.; Monti, J. P. PVPP-polyphenol complexes: A molecular approach. Journal of Agricultural and Food Chemistry 2006, 54, 4383-4389.

41. Monsanto, M.; Hooshyar, N.; Meuldijk, J.; Zondervan, E. Modeling and optimization of green tea precipitation for the recovery of catechins. Separation and Purification Technology 2014, 129, 129-136.

42. Hu, J.; Zhou, D.; Chen, Y. Preparation and antioxidant activity of green tea extract enriched in epigallocatechin (EGC) and epigallocatechin gallate (EGCG). Journal of Agricultural and Food Chemistry 2009, 57, 1349-1353.

43. Huang, K.; Tian, H.; Gai, L.; Wang, J. A review of kinetic models for inactivating microorganisms and enzymes by pulsed electric field processing. Journal of Food Engineering 2012, 111, 191-207.

44. Knorr, D.; Geulen, M.; Grahl, T.; Sitzmann, W. Food application of high electric field pulses. Trends in Food Science \& Technology 1994, 5, 71-75. 
45. Knorr, D.; Angersbach, A. Impact of high-intensity electric field pulses on plant membrane permeabilization. Trends in Food Science \& Technology 1998, 9, 185-191.

46. Zderic, A.; Zondervan, E.; Meuldijk, J. Breakage of cellular tissue by pulsed electric field: Extraction of polyphenols from fresh tea leaves. Chemical Engineering Transactions 2013, 32, 1795-1800.

47. Zhao, J.; Slaga, T. J. Processing method for manufacturing black tea and an improved black tea. 2004. 
Summary 
The oxidation process occurring in green tea leaves during black tea manufacturing is a merely uncontrolled process, in which the oxidative enzymes and tea leaf catechins play the main role. The resulting oxidation products are very important for the flavour and other quality attributes of tea. Much research has been conducted towards the different phenolic oxidation products present in black tea and new compounds are still discovered. Surprisingly enough, the oxidative enzymatic activities responsible for the different compounds have hardly been investigated. When the enzymatic activities could be added separately during tea manufacturing, the oxidation of tea leaves towards a black tea might become more controlled in that specific groups of oxidation products are accumulated. More control of the final phenolic oxidation products in a black tea would be desirable, because subtle differences in the phenolic profile can already lead to changes in the sensory aspects of a tea. Therefore, the aim of this thesis is to understand the enzymatic oxidation reactions occurring in tea oxidation, and to enable rapid analysis of complex mixtures of phenolics without prior preparative fractionation steps.

Chapter 1 provides background information on the composition of tea leaves and the fermentation process of tea. The main green tea phenolics and the major oxidation products are presented, after which the 'oxidative cascade hypothesis' is introduced. The 'oxidative cascade hypothesis' subdivides the oxidation reactions in tea into four levels, of which the first, oligomerization, and second, hydroxylation, are covered in this thesis. An overview of the oxidative enzymes (possibly) present in tea leaves is given. Subsequently, the analysis of black tea, a complex mixture of phenolics, is outlined. Finally, the sensory characteristics of tea, as well as how the different phenolic structures can influence these characteristics are discussed.

In Chapter 2, it was shown that by employing tyrosinase and laccase, both from Agaricus bisporus, on green tea catechins, the oxidation process was directed towards a higher theaflavin (TF) content, which is considered an important quality parameter in tea. The main tea catechins were incubated with tyrosinase and laccase. The product formation was monitored by RP-UHPLC-PDA-ESI-MS. The kind of catechin, their substitution with a galloyl-group, and the type of oxidase used were important factors determining theaflavin concentrations. In particular, incubation of epicatechin with epigallocatechin with tyrosinase gave a high, stable TF content. In a green tea extract, tyrosinase increased the proportion of theaflavins by two-fold compared to a black tea prepared from those leaves by the endogenous enzymes. Laccase incubation mainly resulted into insoluble complexes. These results indicated that the phenolic profile of tea can be modulated by using exogenous oxidases.

Chapter 3 compares three rapid MS screening methods for their ability to annotate twostep-oxidation products in black teas without the need of prior fractionation: (i) full MS, (ii) MS/MS on selected ions, and (iii) selected reaction monitoring (SRM), the first two in 
combination with post analysis extracted ion chromatography (EIC). A model system of $\mathrm{TF}$, epicatechin (EC) and tyrosinase was used to prepare the two-step-oxidation product theatridimensin (T3D), consisting of three oligomerized catechin subunits. The $\mathrm{MS}^{2}$ fragmentation pattern of T3D was compared with that of an isomeric catechin trimer from black tea, TFsEC. $\mathrm{MS}^{2}$ signature fragments were found to distinguish the two isomers, i.e. $\mathrm{m} / z 617$ for T3D and $\mathrm{m} / z 563$ for TFsEC. When exploring black tea for the occurrence of such two-step-oxidation products, the use of MS/MS on selected ions combined with EIC proved to be the most suitable. It not only enabled monitoring $\mathrm{MS}^{2}$ of compounds present in low abundance, it also provided a complete $\mathrm{MS}^{2}$ data set, thereby facilitating the discovery of other isomers, for example theaflavate A. The occurrence of T3Ds and T3Dgs in various black teas was shown for the first time and the 'oxidative cascade hypothesis' was enriched with novel oxidation products.

In Chapter 4, dehydrocatechins (DhC), oligomeric oxidation products of epicatechins, were formed in model incubations of epicatechin with mushroom tyrosinase. DhC oligomers up to tetramers were detected by RP-UHPLC-MS analysis. Measurements with MALDI-TOF-MS showed formation of oligomers up to at least $15 \mathrm{EC}$ subunits. Isomeric DhCs were obtained and a method based on $\mathrm{MS}^{2}$ fragment ratios was set up to distinguish the different interflavanic configurations of the isomers. In the model incubation, eight dehydrodicatechins $\left(\mathrm{DhC}_{2}\right)$ and 22 dehydrotricatechins $\left(\mathrm{DhC}_{3}\right)$ were tentatively annotated by their $\mathrm{MS}^{2}$ fragments. Three different interflavanic configuration-types were annotated for the $\mathrm{DhC}_{2} \mathrm{~s}$. $\mathrm{DhC}_{2} \mathrm{~s}$ and $\mathrm{DhC}_{3} \mathrm{~s}$ were shown to occur in a black tea extract for the first time. For the $\mathrm{DhC}_{2} \mathrm{~s}$, at least two isomeric types, i.e. $\mathrm{DhC} \beta$ and $\mathrm{DhC} \varepsilon$, could be annotated in black tea.

Chapter 5 aimed to identify the enzymatic activity in tea leaves responsible for the hydroxylation step in the tea oxidation process. Two main enzyme activities, peroxidase (POD) and polyphenol oxidase (PPO), were shown to occur in tea leaves, the former of which was hypothesized to be responsible for hydroxylation. A model system with horseradish POD and mushroom tyrosinase was used to investigate hydroxylation of TFs. POD was found capable of hydroxylation, whereas PPO was not. TFs with up to five extra hydroxyl groups were annotated by LC-MS, using their $\mathrm{MS}^{2}$ data. Hydroxylation by POD was also shown for other oligomeric phenolics, such as theanaphtoquinones (TNQs), T3Ds and $\mathrm{DhC}_{2} \mathrm{~s}$. The $\mathrm{H}_{2} \mathrm{O}_{2}$ concentration influenced the extent of hydroxylation. At $0.01 \mathrm{mM}$ $\mathrm{H}_{2} \mathrm{O}_{2}$ hydroxylation occurred, while at higher $\mathrm{H}_{2} \mathrm{O}_{2}$ concentrations the extent of hydroxylation decreased. Oligomeric phenolics with extra hydroxyl groups were evidenced in a black tea as well, by using a selective screening method with RP-UHPLC-MS. TFs with up to five extra hydroxyl groups and traces of other oligomeric catechins with extra hydroxyl groups could be identified by their $\mathrm{MS}^{2}$ data from a consumer black tea product, without any further sample pre-treatment. 
Chapter 6 combines the information described in previous chapters. This resulted in an extended version of the 'oxidative cascade hypothesis', in which not only the newly found oxidation products in tea were incorporated, but also the enzymatic activities were included. The prospects and limitations of the use of model systems compared to use of specific, isolated phenolic compound fractions from black tea are discussed, as well as the annotation of compounds by their signature fragments. A nomenclature was proposed to classify the large variety in tea phenolic oxidation products systematically, the basis of which are the different interflavanic configurations. The extended knowledge on tea oxidation was extrapolated to the effect of the phenolic compounds composition on the sensory characteristics in a final tea. TF, TNQ, T3D, DhC $\delta$ and $\mathrm{DhC} \varepsilon$ were tested for their binding affinity with $\beta$-casein, as an indication for astringency. T3D showed the highest binding affinity of this set. The effect of the hydroxylation reaction on bitterness was discussed as well. The future prospects for applications of controlled tea oxidation in tea processing were discussed. Mild disclosure of both enzymes and phenolics from tea leaves is considered to be important. Subsequent fractionation of these two tea constituents into individual enzymes or catechins is expected to result in the optimal control over the oxidation process. 
Acknowledgements 
The past 4.5 years not only have resulted in this thesis booklet, but also in my personal growth and gaining a lot of friends. All of which I, of course, wouldn't have managed alone. Hence, I want to use these pages to acknowledge everybody that supported me throughout my thesis-project. In my opinion, the best way to show appreciation is in the form of a 'stukje', but unfortunately that doesn't fit the format of a booklet. Alternatively, I'll give you just the division in 'stukje'-parts, including the songs you have to listen to while reading, only the animated powerpoints and masked actors you'll have to imagine ;).

\section{Oligomerization of my knowledge on tea}

(The photosynthesis song - Peter Weatherall)

Allereerst wil ik uiteraard Jean-Paul en Harry bedanken, die het vertrouwen in mij hadden dat ik dit thee-project tot een goed einde kon brengen. Jean-Paul, jouw onuitputtelijke pogingen om mijzelf daar ook van te overtuigen, hebben mij enorm doen groeien, waardoor ik nu ook (heel onbescheiden ;)) met trots naar mijn thesis kan kijken. Ook de besprekingen met jou waardeerde ik heel erg, de lange discussies waarna ik altijd met een vertrouwen en enthousiasme boost weer verder kon. Harry, de manier waarop jij FCH runt stel ik heel erg op prijs. Bijvoorbeeld, om het mij, op mijn eerste werkdag als AIO, te verbieden je nog met ' $u$ ' aan te spreken. Ook het saamhorigheidsgevoel dat we hier hebben met de collega's is mede aan jou te danken, door het strijden voor een koffiekamer en door de sociale activiteiten te stimuleren. Beiden ook bedankt voor jullie kritische en objectieve blik op mijn verhalen. Ook al ging het me vaak aan het hart om mijn 'wollige' tekst te schrappen, de verhalen zijn er zeker beter en duidelijker op geworden.

Mijn project was deel van een groter geheel, samengevoegd door ISPT. Ik wil van ISPT iedereen bedanken die me heeft geholpen, met name Daniëlla, die me altijd weer verder hielp bij problemen. I would like to thank all the participants of our 'Mild disclosure' project, thank you for the input during the presentations and discussions at the project meetings. Special thanks to the different project leaders of our project, Olivera, Nasim and Ardjan, who kept everyone on track and up-to-date. My fellow PhD-ladies in the project, Alexandra, Aleksandra and Laura, thank you for the nice discussions and good times we had.

During my PhD I also supervised BSc and MSc students. Supervising students was for me every time a new learning experience. Robin, Eline, Robbin, Wei, Marieke, Biyuan and Laura, thank you for working on my project and for the nice times in the lab.

Alle technicians bedankt voor het draaiende houden van de apparatuur, alle introducties en het bestellen van de chemicaliën en disposables. Met name Mark, bedankt voor alle hulp met de UPLC-MS, van het steviger vast draaien van de kolom tot hulp bij de methode ontwikkeling met de MS-settings. 
All Phyto's; Carlos, Tomas, Maxime, Wibke, Yannick, Milou, Aisyah, Ya, Carla, Anne, Renske, Wouter and Silvia, I really enjoyed being part of the team and sharing the lab with you. Thank you for all discussions, experiences and ideas shared at the Phyto-meetings.

Everyone else at the Laboratory of Food Chemistry, thank you for the discussions, answers and occasional help in the lab, in particular all strong (wo)men that helped me opening $\mathrm{ACN}$ bottles and loosening centrifuge rotors;).

\section{Hydroxylation - adding something more to a working day}

((I've had) the time of my life - Bill Medley \& Jennifer Warnes)

Going to the lab meant more for me than just going to work. I met a lot of special people that added some flavour and 'gezelligheid' to the day, which I appreciated a lot!

First of all my project-partner and office mate, Alexandra, thank you for all the times you listened to my frustrations and for the chocolates provided when needed. But of course, also a lot of thanks for all the fun times and conversations we shared, I will never forget them ;). I am very glad you will sit next to me as my paranymph during my defence.

Wouter, om een bepaalde reden, ik weet nog steeds niet welke, klikte het meteen heel goed met jou toen je hier als AIO kwam werken. Ik wil je bedanken dat je de laatste anderhalf jaar van mijn thesis hebt opgevrolijkt met onze, wat zal ik zeggen, meest interessante gesprekken. Ik ben blij dat jij mij ook vergezeld als paranimf op de grote dag.

Tomas, behalve mijn grote voorbeeld ;), was je er ook altijd voor vragen of gewoon om een praatje te houden. Ik heb genoten van je aanwezigheid, vooral in combinatie met Maxime was het goed voor de lachspieren (-). Bedankt voor de leuke tijd bij FCH en de snackxperiences die je me hebt bijgebracht.

Milou, bedankt voor alle gezelligheid binnen en buiten het lab. Ik ben blij dat ik altijd bij jou terecht kan in goede en slechte tijden. En bedankt voor je goede zorgen, zoals het voor mij koken tijdens de laatste drukke weken. En dan is er ook altijd nog 'De Dromendans' waardoor we alles weer positief gaan zien $:-$.

Geralt, ook al was je maar een jaar bij $\mathrm{FCH}$, toch heb je een onuitwisbare indruk achtergelaten, met je bloemetjes shirts en chocopasta. Bedankt voor je tegendraadse mening die altijd weer voor hilariteit zorgde ;).

Jolanda, jij was er altijd voor de non-scientific vragen, of gewoon als ik even ergens over moest klagen of weer een snoepje kwam halen. Bedankt voor het geven van kleur (letterlijk $\odot$ ) en sfeer aan de afdeling!

Special thanks to my office-mates throughout the years (and buildings). First of all, Surender and Red, we never wanted to kick you out! ;) I really enjoyed having you guys in the office $(-)$. Then we changed to an all-girl office, Alexandra, Edita, Suzanne and Bianca, 
thank you for sharing celebrations, frustrations and chocolates in the office. I appreciate that you made me pretend I was not the only one finishing the cookies and chocolates in the drawer ;).

There are many more colleagues I would have liked to thank by name, but I was trying (yet failing) to keep it short. Fact is, I really enjoyed all the years at the Laboratory of Food Chemistry, and I will really miss it! I want to thank everybody for the 'gezelligheid' during coffee and lunch breaks, the commitment in making 'stukjes' and the drinks and dinners at Passantje ;). I hope we will keep in contact!

\section{The exogenous oxidative enzymes that kept oxidizing me outside work}

(Let it go - Idina Menzel (Frozen))

There are also some people outside work I have to thank for providing me with the necessary support and/or relaxation to continue with my research.

Christiane, thanks for convincing me to try the painting course, it was the best decision ever! Annuska, bedankt voor jouw unieke, vrije manier van schilderles geven en alle gezelligheid tijdens het schilderen. Ik kijk er elke week weer naar uit! Beide ook bedankt voor jullie kritische, creatieve blik op mijn thesis cover $(-)$.

Volleybal gaf me een mooie kans om alle problemen en frustraties omtrent werk eruit te slaan. Ik wil mijn team (inclusief trainer en coach) bedanken voor alle sportiviteit en gezelligheid, de koffie en theetjes van tevoren, bier en AA-tjes achteraf en natuurlijk de feestjes! In het bijzonder mijn 'Hamburger met frietjes' dames, Annemarie, Daniëlle, Elja en Linda, bedankt voor al die slappe-lach buien, Singstar-avonden en KvK/Disneyconcerten in de douches $:$.

Marijke, jij ook heel erg bedankt voor je support, kaartjes en je onuitputtelijke pogingen om toch af en toe eens af te kunnen spreken $(-)$, ook al was ik nogal traag met reageren en is het excuus 'te druk' erg vaak langsgekomen. Maar het is altijd weer vanouds gezellig $(;)$.

Papa, Mamma, Lisa, Maaike en Urtzi, bedankt voor de steun en interesse voor mijn onderzoek. Maar vooral ook dank voor het begrip als ik het er liever niet over wilde hebben. Ik weet dat ik altijd op jullie kan rekenen, maar ik ben ook een beetje eigenwijs.

Ricardo, you are the best thing that I (indirectly) got out of this thesis - . . Thank you for your support, help and patience during the last period of my thesis, now we can totally focus on our life together.

\section{Annewieke}


About the author 


\section{Curriculum vitae}

Johanna Willemina (Annewieke) Verloop was born on March $4^{\text {th }}$ 1988 in Mijdrecht, The Netherlands. After graduating from high school (Veenlanden College, Mijdrecht) in 2006, she started her studies Food Technology at Wageningen University. Her BSc degree was completed (cum laude) with a thesis on the characterization of arabinoxylans of different wheat varieties at the Laboratory of Food Chemistry. Her MSc degree in Food Technology, with a specialization in Ingredient Functionality, was completed (cum laude) with a thesis on the characterization of the products of the

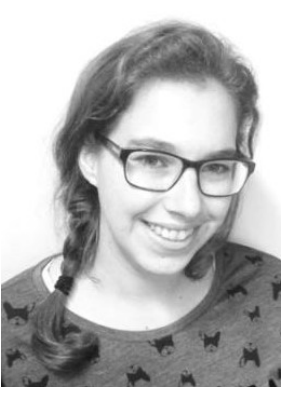
polyphenol oxidase-catalysed reaction of chlorogenic acid with sulphite at the Laboratory of Food Chemistry. An industrial internship was performed at H.J. Heinz (Nijmegen, The Netherlands), focussing on the technological and sensory aspects of chocolate sprinkles and the effects of changes in the ingredients. In September 2011 Annewieke started her PhD at the Laboratory of Food Chemistry of Wageningen University on the project "Mild disclosure - Selective opening and fractionation of natural raw materials". Her project was sponsored by the Institute for Sustainable Process Technology (ISPT) in cooperation with several industrial partners, such as Unilever, Heineken, Cosun, DSM and Synthon. The results of her work are described in this thesis.

Contact: annewieke.verloop@gmail.com 


\section{List of publications}

Kuipers, T.F.M., Narváez-Cuenca, C.-E., Vincken, J.-P., Verloop, A.J.W., Van Berkel, W.J.H., Gruppen, H. Inhibition of enzymatic browning of chlorogenic acid by sulfurcontaining compounds. Journal of Agricultural and Food Chemistry, 2012, 60, 3507-3514.

Verloop, A.J.W., Gruppen, H., Bisschop, R., Vincken, J.-P. Altering the phenolics profile of a green tea leaves extract using exogenous oxidases. Food Chemistry, 2016, 196, $1197-$ 1206.

Verloop, A.J.W., Vincken, J.-P., Gruppen, H. An MS/MS method based on selected ions detects low abundance phenolics in black tea - theatridimensins as product of the oxidative cascade. Submitted to Rapid Communications in Mass Spectrometry.

Verloop, A.J.W., Gruppen, H., Vincken, J.-P. Structural characterization of different dehydrocatechin oligomers by MS/MS and their occurrence in black tea. Submitted to Journal of Agricultural and Food Chemistry.

Verloop, A.J.W., Vincken, J.-P., Gruppen, H. Peroxidase is responsible for the hydroxylation step in the 'oxidative cascade hypothesis' occurring during processing of black tea. To be submitted. 
About the author

\section{Overview of completed training activities}

\section{Discipline specific activities}

Courses

- $\quad$ Reaction kinetics in food science (VLAG), Wageningen, The Netherlands, 2012

- $\quad$ Advanced food analysis (VLAG), Wageningen, The Netherlands, $2013^{\mathrm{a}}$

- $\quad$ Masterclass on Applied Biocatalysis (VLAG/GBB), Wageningen, The Netherlands, 2014

\section{Conferences}

- $\quad 26^{\text {th }}$ International conference on polyphenols, Florence, Italy, 2012

- $\quad 6^{\text {th }}$ European workshop on leaf senescence, Versailles, France, $2013^{\mathrm{a}}$

- Mini symposium WBox2: Redox biocatalysis, Wageningen, The Netherlands, 2013

- $\quad 7^{\text {th }}$ Oxyzymes conference, Vienna, Austria, 2014

- $\quad 3^{\text {rd }}$ CoCoTea conference, Aveiro, Portugal, 2015

\section{General courses}

- $\quad$ VLAG PhD introduction week (VLAG), 2012

- $\quad \mathrm{PhD}$ competence assessment (WGS), 2012

- $\quad$ Teaching and supervising thesis students (ESD), 2012

- Interpersonal communication for PhD students (WGS), 2013

- $\quad$ Project and time management (WGS), 2013

- $\quad$ Techniques for writing and presenting a scientific paper (WGS), 2013

- $\quad$ Scientific writing (WUR In’to Languages), 2014

\section{Additional activities}

- $\quad$ Preparation of $\mathrm{PhD}$ research proposal

- $\quad$ PhD trip FCH to Singapore and Malaysia, 2012,

- $\quad$ PhD trip FCH to Germany, Denmark, Sweden and Finland, 2014,

- $\quad \mathrm{BSc} / \mathrm{MSc}$ thesis student presentations and colloquia, 2011-2015

- $\quad$ PhD presentations Food Chemistry, 2011-2015

\footnotetext{
${ }^{\mathrm{a}}$ Poster, ${ }^{\mathrm{b}}$ Oral presentation

VLAG: Graduate school for Nutrition, Food Technology, Agrobiotechnology and Health Sciences

GBB: Groningen Biomolecular Sciences and Biotechnology Institute

WGS: Wageningen Graduate School

ESD: Educational Staff Development

WUR: Wageningen University and Research Centre

FCH: Laboratory of Food Chemistry
} 
The work described in this thesis was performed at the Laboratory of Food Chemistry, Wageningen University, The Netherlands. This research was sponsored by the Institute for Sustainable Process Technology (ISPT), project number: FO-10-06.

Financial support from Wageningen University for printing this thesis is gratefully acknowledged. 
This thesis was printed by Gildeprint, Enschede, The Netherlands Edition: 275 copies

Cover design: Annewieke J.W. Verloop

Annewieke J.W. Verloop, 2016

146 